\title{
Coordinated Multi-Agents Patrolling Algorithms
}

by

Najmeh Taleb

A thesis submitted to the Faculty of Graduate and Postdoctoral Affairs in partial fulfillment of the requirements for the degree of

Doctor of Philosophy

in

Computer Science

CARLETON UNIVERSITY

OTTAWA, ONTARIO

(C) 2016

Najmeh Taleb 
To my angels, dad and mom, and my love, Aali 


\section{Abstract}

We introduce and study various patrolling algorithms using mobile robots. A team of $k$ mobile robots (patrolmen), is deployed on a weighted graph $G$, in which edge weights represent distances. The robots perpetually move along the domain not exceeding their maximal speed. The robots need to patrol the graph by regularly visiting all points of the domain. The goal of the patrolling problem is to find the perpetual movement of the robots minimizing idle time, which is the maximal time when a point of the graph remains unseen by any robot. In this thesis, we investigate various versions of patrolling problems, in each case attempting to optimize the idle time.

In the first scenario, we consider a case where at most $f$ of $k$ robots may be faulty (unreliable), i.e., they do not report their monitoring activities. We design an optimal algorithm for the open curves (segments), and then use these results to study the case of general graphs. We also propose an optimal patrolling strategy for Eulerian graphs. Afterward, we show that computing idle time for three robots, at most one of which is faulty, is NP-hard for some general graphs.

Next, we study the patrolling problem by reliable robots, but equipped with distinct visibility ranges, i.e., every robot $i$ has a range of visibility $r_{i}$ representing the distance from its current position, at which the robot can see in each direction. We give the optimal patrolling algorithms for the case of close curves (cycles) and open curves (segments), when all robots have the same maximal speed and different visibility ranges. We also briefly discuss the case where robots have distinct speeds and visibility ranges to show that patrolling by robots equipped with visibility is entirely different than the case of robots with zero visibility. Moreover, we show that patrolling general graphs by robots with the same speed and distinct visibility ranges is NP-hard.

Finally, we consider patrolling trees by a team of mobile robots with the same speed and zero visibility. We theoretically show the optimality of an off-line centralized algorithm for trees patrolling. Then, we use these results to experimentally show the efficiency of an on-line distributed algorithm (known as rotor-router) for trees patrolling. 


\section{Acknowledgements}

I am incredibly indebted to Dr. Evangelos Kranakis and Dr. Jurek Czyzowicz for their continuous support, thoughtful advice and warm encouragements throughout my doctoral program. I acquired an invaluable wealth of knowledge under their supervision and learned how to approach a research problem effectively. They have always inspired me as true scientists. I sincerely enjoyed the experience of working with them which trained me for my future career and life.

I would also like to thank everyone who has collaborated on the research presented in this thesis. 


\section{Contents}

$\begin{array}{ll}\text { Abstract } & \text { ii }\end{array}$

$\begin{array}{ll}\text { Acknowledgements } & \text { ii }\end{array}$

List of Algorithms vi

List of Figures $\quad$ vii

List of Tables $\quad$ viii

1 Introduction 1

1.1 Motivation to Study Patrolling Problem . . . . . . . . . . . 2

1.2 Our Model and Assumptions . . . . . . . . . . . . . . . . 6

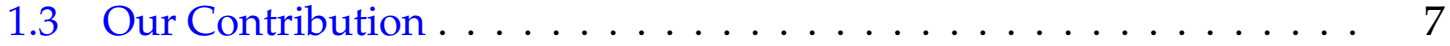

1.4 Thesis Outline . . . . . . . . . . . . . . . . . . . . . . 9

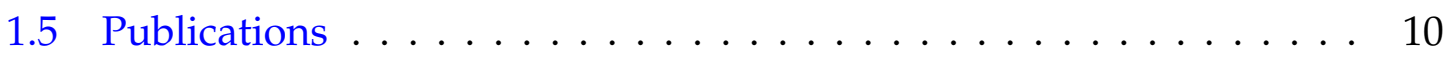

2 Related Work 11

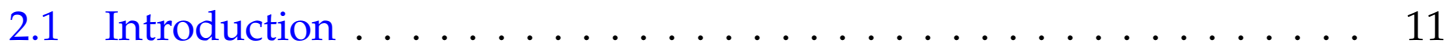

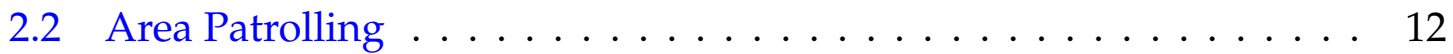

2.3 Perimeter Patrolling . . . . . . . . . . . . . . . . . . 17

2.4 Randomized Patrolling . . . . . . . . . . . . . . . . . 22

2.5 Distributed Patrolling . . . . . . . . . . . . . . . . 23

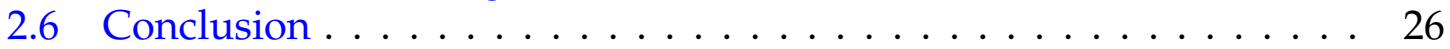

3 Patrolling Graphs by Faulty Mobile Robots 28

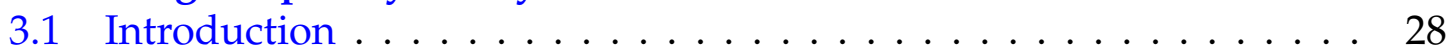

3.1.1 Preliminaries and Notation . . . . . . . . . . . . . . . . . . 29

3.1.2 Outline and Results . . . . . . . . . . . . . . . . . . 31

3.2 Open Fence Patrolling . . . . . . . . . . . . . . . . . . 31

3.2.1 The Upper Bound . . . . . . . . . . . . . . . . . . . . . . 32

3.2.2 The Lower Bound . . . . . . . . . . . . . . . . . . . . . . . . . . 35

3.3 Arbitrary Graphs Patrolling . . . . . . . . . . . . . . . . . . 37

3.3.1 A General Result and Algorithm . . . . . . . . . . . 38 
3.3.2 Hardness of Computing the Idleness . . . . . . . . . . . . . 39

3.3.3 Characterizing Graphs with Minimum Idle Time . . . . . . . . 44

3.4 Conclusion and Open Problems . . . . . . . . . . . . 46

4 Patrolling Graphs by Mobile Robots Equipped with Visibility 47

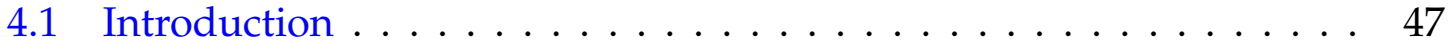

4.1 .1 Preliminaries and Notation . . . . . . . . . . . . . . 48

4.1 .2 Outline and Results . . . . . . . . . . . . . . . . . 49

4.2 Closed Fence Patrolling . . . . . . . . . . . . . . . . . . 50

4.2.1 Robots with Equal Speeds . . . . . . . . . . . . . . 50

4.2.2 Robots with Different Speeds . . . . . . . . . . . . . . 52

4.3 Open Fence Patrolling . . . . . . . . . . . . . . . . . . . . 53

4.3.1 Robots with Equal Speeds . . . . . . . . . . . . . . . 53

4.3.2 Robots with Different Speeds . . . . . . . . . . . . . . 61

4.4 Arbitrary Graphs Patrolling . . . . . . . . . . . . . . . . . . 63

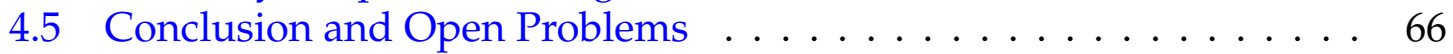

5 Patrolling Trees by Homogeneous Mobile Robots $\quad 68$

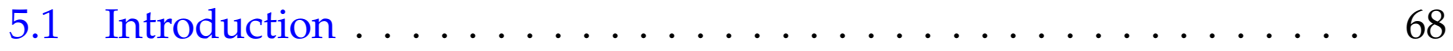

5.1 .1 Preliminaries and Notation . . . . . . . . . . . . . . . . . 69

5.1 .2 Outline and Results . . . . . . . . . . . . . . . . . . . 69

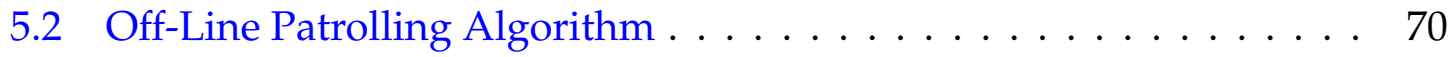

5.2.1 Patrolling of Geometric Trees . . . . . . . . . . . . 70

5.2.2 Patrolling of Graph Trees . . . . . . . . . . . . . . 76

5.3 On-Line Patrolling Algorithm with Rotor-Router . . . . . . . . . . 80

5.3.1 Lower Bounds . . . . . . . . . . . . . . . . . . . . . . 82

5.3 .2 Experimental Results . . . . . . . . . . . . . . . . 85

5.4 Conclusion and Open Problems . . . . . . . . . . . . . 94

6 Rotor-Router Simulator $\quad 97$

6.1 Introduction . . . . . . . . . . . . . . . . . 97

6.1.1 Preliminaries and Notation . . . . . . . . . . . . . . . 97

6.2 Design of Rotor-Router Simulator . . . . . . . . . . . . . 100

6.3 Generating Uniform Random Trees . . . . . . . . . . . . . . . . 103

6.4 Generating Uniform Random Initial Configurations . . . . . . . . . 104

6.5 Applying Rotor-Router . . . . . . . . . . . . . . . . . . . . 106

6.6 Computing Idle Time . . . . . . . . . . . . . . . . . . . . 110

6.7 Conclusion and Open Problems . . . . . . . . . . . . 111 
7 Conclusion and Future Work 113

7.1 Conclusion . . . . . . . . . . . . . . . . . . 113

7.2 Future Work . . . . . . . . . . . . . . . . . . 120

$\begin{array}{ll}\text { Appendices } & 123\end{array}$

A Rotor-Router Experimental Results for a Random Tree 124

B Rotor-Router Experimental Results for 50 Random Trees 129

C Rotor-Router Experimental Results for a Random Clover Tree 134

D Rotor-Router Experimental Results for a Tripod Tree 143

$\begin{array}{ll}\text { Abbreviations } & 152\end{array}$

$\begin{array}{ll}\text { Nomenclature } & 153\end{array}$

$\begin{array}{ll}\text { Bibliography } & 155\end{array}$

$\begin{array}{ll}\text { Index } & 174\end{array}$ 


\section{List of Algorithms}

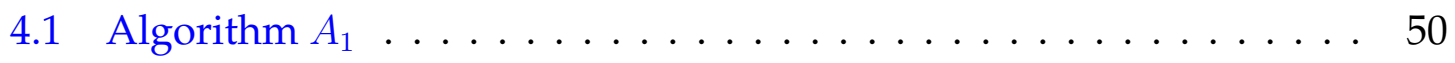

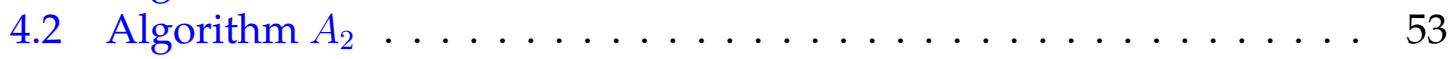

4.3 Construction of Mobile Intervals . . . . . . . . . . . . . . 56

4.4 Algorithm $A_{3} \ldots \ldots \ldots \ldots \ldots \ldots \ldots \ldots \ldots \ldots \ldots \ldots \ldots$

5.1 Single Rotor-Router . . . . . . . . . . . . . . . . . . . 81

5.2 Parallel Rotor-Routers . . . . . . . . . . . . . . . . . . . . . . . . . 81

6.1 Rotor-Router Simulator . . . . . . . . . . . . . . . . . . . . . . . . 102

6.2 Aldous-Broder Algorithm . . . . . . . . . . . . . . . . 103

6.3 Generating Random Tree . . . . . . . . . . . . . . . . . . . . . . 104

6.4 Generating Random Initial Configuration . . . . . . . . . . . . 105

6.5 Running Rotor-Router . . . . . . . . . . . . . . . . . . . . 107

6.6 Robots Movement Following Rotor-Router . . . . . . . . . . . . . 109

6.7 Computing Idle Time . . . . . . . . . . . . . . . . . . . . 110 


\section{List of Figures}

3.1 In the Eulerian cycle the robots are moving in the same direction, but from the "point of view of the point $p$ " the traversal is in opposite directions . . . . . . . . . . . . . . . . . . 34

3.2 Gadget $X$ used in the construction of graph $H$ in the hardness proof. 42

3.3 Assignment of edges of gadget $X^{(j)}(e) \subseteq H$ to sets $E_{1}, E_{2}, E_{3}$ for an edge $e$ having color $i$. Edges marked $i$ in the figure belong to $E_{i}$, edges marked $i+1$ belong to $E_{(i \bmod 3)+1}$, and edges marked $i+2$ belong to $E_{((i+1) \bmod 3)+1} \ldots \ldots \ldots \ldots \ldots \ldots \ldots \ldots \ldots \ldots \ldots \ldots$

4.1 Positions of robots in the algorithm $A^{\text {*is }} \ldots \ldots \ldots \ldots \ldots$

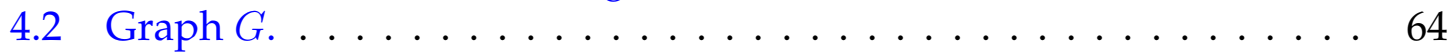

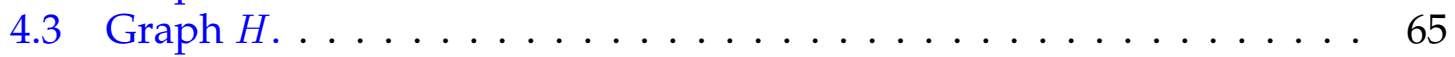

5.1 Proving a $4 / 3$ lower bound for patrolling the star graph by two robots $r, r^{\prime} \ldots \ldots \ldots \ldots \ldots \ldots \ldots \ldots \ldots$

5.2 (a) cover time, (b) idle time, (c) lock-in time, and (d) periodicity, of rotor-router algorithm for a random tree with 50 nodes, and 100 distinctive initial configurations for each set of $k=1,2, \ldots, 50$ robots, drawn against the number of participating robots in each experiment. 87

5.3 (a) cover time, (b) idle time, (c) lock-in time, and (d) periodicity of rotor-router algorithm on 50 random trees with 50 nodes, and 100 distinctive initial configurations for each set of $k=1,2, \ldots, 50$ robots, drawn against the number of participating robots in each experiment. 88

5.4 (a) cover time, (b) idle time, (c) lock-in time, and (d) periodicity of rotor-router algorithm for a Tripod tree with 106 nodes, and 100 random initial configurations for each set of $k=1,2, \ldots, 105$ robots, drawn against the number of participating robots. . . . . . . . . .

5.5 (a) cover time, (b) idle time, (c) lock-in time, and (d) periodicity of rotor-router algorithm for a random Clover tree with 106 nodes, and 100 random initial configurations for each set of $k=1,2, \ldots, 105$ robots, drawn against the number of participating robots. . . . . . 95

6.1 A snapshot of rotor-router simulator $\ldots \ldots \ldots \ldots$. . . . . . . 101 


\section{List of Tables}

A.1 Cover times obtained by applying rotor-router on a random tree with 50 nodes, and 100 distinctive initial configurations for each set of $k=1,2 \ldots .50$ robots. . . . . . . . . . . . . . . . . . . . . 125

A.2 Lock-in times obtained by applying rotor-router on a random tree with 50 nodes, and 100 distinctive initial configurations for each set

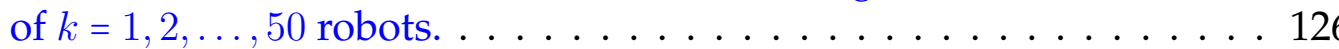

A.3 Periodicities obtained by applying rotor-router on a random tree with 50 nodes, and 100 distinctive initial configurations for each set of $k=1,2, \ldots, 50$ robots. . . . . . . . . . . . . . . . . . . . 127

A.4 Idle times obtained by applying rotor-router on a random tree with 50 nodes, and 100 distinctive initial configurations for each set of $k=1,2, \ldots, 50$ robots. $\ldots \ldots \ldots \ldots \ldots \ldots \ldots \ldots \ldots$

B.1 Cover times obtained by applying rotor-router on 50 random trees with 50 nodes, and 100 distinctive initial configurations for each set

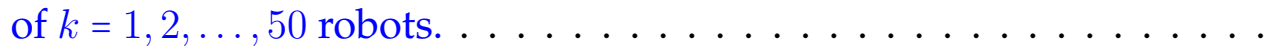

B.2 Lock-in times obtained by applying rotor-router on 50 random trees with 50 nodes, and 100 distinctive initial configurations for each set

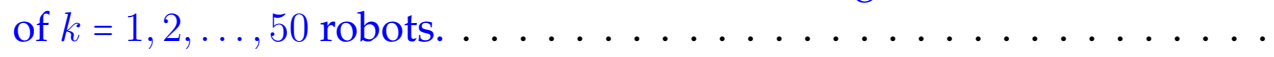

B.3 Periodicities obtained by applying rotor-router on 50 random trees with 50 nodes, and 100 distinctive initial configurations for each set

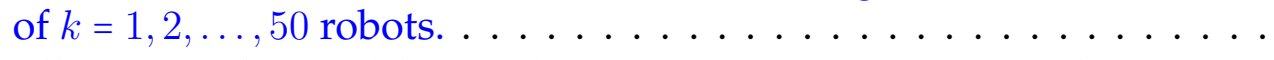

B.4 Idle times obtained by applying rotor-router on 50 random trees with 50 nodes, and 100 distinctive initial configurations for each set

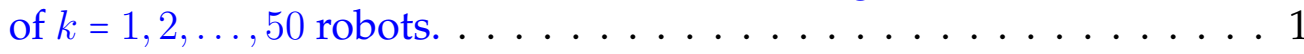

C.1 Cover times obtained by applying rotor-router on a random Clover tree with 106 nodes, and 100 random initial configurations for each set of $k=1, \ldots, 52 . \ldots \ldots \ldots \ldots \ldots \ldots \ldots \ldots \ldots \ldots \ldots$

C.2 Cover times obtained by applying rotor-router on a random Clover tree with 106 nodes, and 100 random initial configurations for each

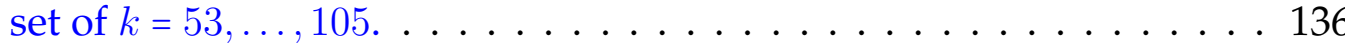


C.3 Lock-in times obtained by applying rotor-router on a random Clover tree with 106 nodes, and 100 random initial configurations for each

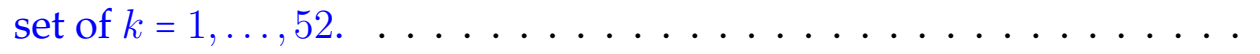

C.4 Lock-in times obtained by applying rotor-router on a random Clover tree with 106 nodes, and 100 random initial configurations for each

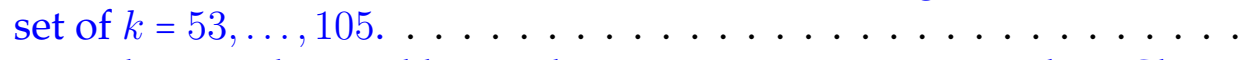

C.5 Periodicities obtained by applying rotor-router on a random Clover tree with 106 nodes, and 100 random initial configurations for each

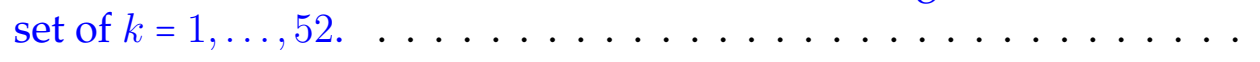

C.6 Periodicities obtained by applying rotor-router on a random Clover tree with 106 nodes, and 100 random initial configurations for each set of $k=53, \ldots, 105 \ldots \ldots \ldots \ldots \ldots \ldots \ldots \ldots \ldots \ldots \ldots \ldots$

C.7 Idle times obtained by applying rotor-router on a random Clover tree with 106 nodes, and 100 random initial configurations for each set of $k=1, \ldots, 52 \ldots \ldots \ldots \ldots \ldots \ldots \ldots \ldots \ldots \ldots \ldots \ldots \ldots \ldots$

C.8 Idle times obtained by applying rotor-router on a random Clover tree with 106 nodes, and 100 random initial configurations for each

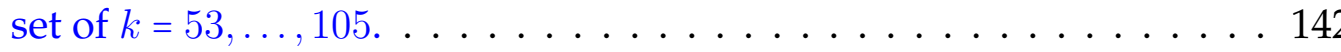

D.1 Cover times obtained by applying rotor-router on a Tripod tree with 106 nodes, and 100 random initial configurations for each set of $k=$

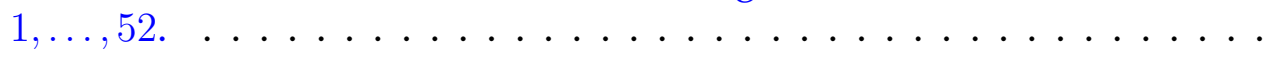

D.2 Cover times obtained by applying rotor-router on a Tripod tree with 106 nodes, and 100 random initial configurations for each set of $k=$ $53, \ldots, 105 \ldots \ldots \ldots \ldots \ldots \ldots \ldots \ldots \ldots \ldots \ldots$

D.3 Lock-in times obtained by applying rotor-router on a Tripod tree with 106 nodes, and 100 random initial configurations for each set

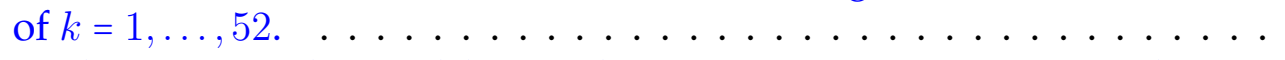

D.4 Lock-in times obtained by applying rotor-router on a Tripod tree with 106 nodes, and 100 random initial configurations for each set

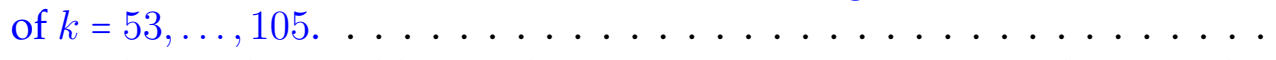

D.5 Periodicity obtained by applying rotor-router on a Tripod tree with 106 nodes, and 100 random initial configurations for each set of $k=$

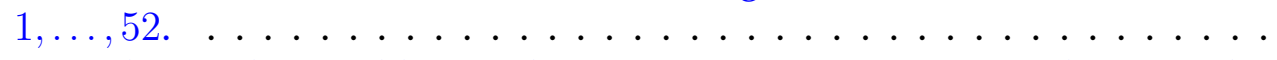

D.6 Periodicity obtained by applying rotor-router on a Tripod tree with 106 nodes, and 100 random initial configurations for each set of $k=$

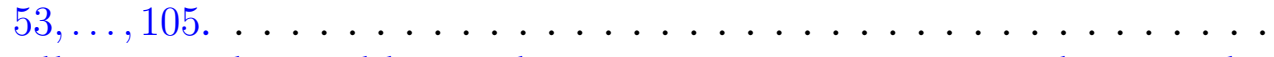

D.7 Idle times obtained by applying rotor-router on a Tripod tree with 106 nodes, and 100 random initial configurations for each set of $k=$

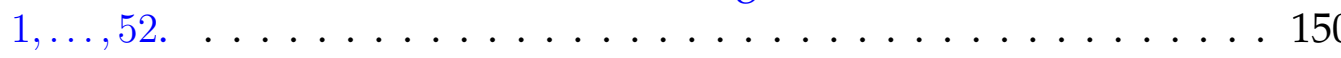


D.8 Idle times obtained by applying rotor-router on a Tripod tree with 106 nodes, and 100 random initial configurations for each set of $k=$

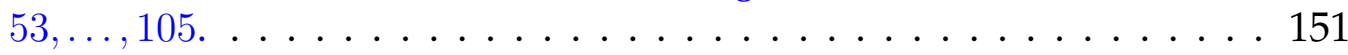




\section{Chapter 1}

\section{Introduction}

Patrolling is defined as the act of walking or traveling around an area, at regular intervals, in order to protect, inspect or supervise the area. Patrolling is by nature a multi-agents task, in which a team of mobile robots can perform it either in a static, or in a dynamically changing environment. Recently, the patrolling problem has been of great interest because of its fundamental importance in many different applications such as security, and surveillance (monitoring). For this purpose, a variety of patrolling models have been studied including deterministic and randomized, as well as centralized and distributed strategies depending on various objectives of patrolling task.

The fundamental measure for evaluating the efficiency of patrolling algorithms is the criterion of idleness, first introduced by Machado et al. [67]. The general idea of idleness is to measure the frequency of visits to the points in the environment by mobile robots $[6,22]$. Depending on the patrolling approach, the idleness is sometimes viewed as the average [29], worst-case [18], probabilistic [3], or experimentally verified [44] time elapsed since the last time a point was visited (see also $[6,23])$. In some studies, this is referred to as the blanket time [96], or refresh time 
[73], so as to indicate a similar measurement of algorithm efficiency.

\subsection{Motivation to Study Patrolling Problem}

The patrolling problem is very interesting from a theoretical perspective, since it is closely connected to very well-known theoretical problems in computer science such as "coverage", "exploration", and "art gallery" problems. Moreover, there are numerous real world applications of patrolling tasks, for instance, monitoring by Unmanned Aerial Vehicles (UAVs), that make it very attractive to study the patrolling problem.

There is a tight connection between the patrolling problem and the multi-robot coverage problem, which is one of the fundamental tasks in computer science (see, e.g., $[40,52,53])$. In both problems (either patrolling or coverage), a team of mobile robots is required to visit every point of a given region. However, the purpose of the coverage algorithms is to minimize the number of visits to each point, while the goal in the patrolling algorithms is to maximize the number of visits. On the other hand, continuous coverage problem (persistent surveillance) (see, e.g., [17, 85, 86]), which is covering a dynamically changing environment perpetually, has a more similar setting to the patrolling problem. In such a setting, robots must repeatedly visit the entire environment to update their observations for possible changes, and the goal is to maximize the number of visits to each point of the environment (the same goal as that of the patrolling algorithms). Another similar setting to the patrolling problem is ongoing exploration of an unknown environment by a group of 
simple mobile robots (see, e.g., [89, 90, 92]). In such a setting, the robots are perpetually visiting the environment in order to monitor, and report possible changes that may occur in the environment.

The patrolling problem may also be viewed as a version of the art gallery prob$l e m$, in which a set of stationary or mobile guards have to protect a given geometric environment (see, e.g., [69, 71, 84]). In the case of stationary guards, in most studies, the goal is to minimize the number of guards required to view the entire environment. This problem is NP-hard, and many approximations and inapproximability results were obtained [41,50]. For the case of mobile guards (often known as the "watchman route problem"), the question of a single watchman has been generally addressed. The optimization criterion is the path length traversed by the watchman, so that every point of the environment is seen from some position on the path. This problem is closely related to the "Traveling Salesman Problem (TSP)", in which for a given list of cities and the distances between each pair of cities, the goal is to find the shortest possible route that visits each city exactly once and returns to the origin city. Unsurprisingly, many general watchman route problems are NP-hard [11, 37, 38]. However, polynomial time algorithmic solutions are available for many specific cases. Other variants of the art gallery problem, known as "zookeeper route", "safari route" and "aquarium keeper", were studied in [23], [70], and [29], respectively.

There is also a wide variety of problems that could be reformulated as particular patrolling tasks. For instance, searching a graph or polygon by a team of mobile robots, which are looking for a stationary or mobile intruder (see, e.g., [47, 95]). 
This problem falls into the vastly investigated domain of cops and robbers [46]. Another similar work to this problem is graph-clear problem, which seeks the minimum number of robots needed to detect all possible intruders in a given complex environment that can be modeled as a graph [62]. These problems (graph searching, and graph clearing problems) are very similar to the patrolling problem considering an adversarial setting, in which an adversary is attempting to penetrate the environment.

Recently, researchers have integrated the patrolling task with other event handling tasks. For instance, Czyzowicz et al. [32] introduced a new problem concerning the exploration of a geometric domain by a team of mobile robots. Considering a line segment, the robots are initially placed at one of its endpoints. Each robot has a searching speed and a walking speed, where searching speed is less than walking speed. A robot either walks along a portion of the segment, or searches within that segment at each time moment. The search of the segment is completed at the time when each of its points has been searched by at least one of the robots. The goal is to search the line segment as fast as possible. Although the proposed problem is to search the line segment only once, it could be extended to perpetual walking and searching, which may be considered as a new type of patrolling problem.

The patrolling problem has been studied extensively not only because of its close connection to fundamental problems in computer science, but also because of numerous real world applications of the patrolling task. Patrolling strategies are suitable for the tasks that require continual execution, such as monitoring of oil spills [25], detection of forest fires [20], keeping track of border changes [87], 
and surveilling an environment [3, 43, 93], or sensitive areas [19]. Recently, monitoring (patrolling) by UAVs has attracted numerous attention. For instance, Ryan et al. [80] and Girard et al. [51] described architectures for multiple robots patrolling using UAVs, in which a single operator coordinates and commands multiple robots (UAVs). In a similar study, Kingston et al. [60] proposed an efficient and distributed solution to the perimeter patrolling problem for robots with no communication tool.

In addition to surveillance, robots can save human lives by replacing humans in dangerous tasks. For instance, robots may be used for removing hazardous waste [54], or working in coal mining industry. Moreover, robots are useful in searching and locating objects or humans that need to be rescued from a disaster zone [1]. Robots may also reduce costs by replacing humans in tedious tasks, for instance, cleaning [26], taking care of elderly people, or monitoring patients. In other scenarios, network administrators may use mobile agents (i.e., software programs) to patrol and detect network failures [10]. In addition, mobile agents could be used to discover web pages which are required to be indexed by search engines [24].

In adversarial settings, where an intruder is attempting to penetrate the environment, for examples, art galleries or banks, patrolling robots may be used for the sake of security. In addition, patrolling strategies proposed for such adversarial settings have huge applications in computer games, where distributed surveillance, inspection or control are required [7, 9]. In these settings, patrolling is considered as a game between patrollers and intruder. 


\subsection{Our Model and Assumptions}

A precise formulation of the patrolling problem requires the characterization of the environment to be patrolled, the robots' capabilities, and the performance criteria. In this section, we clearly explain our model and assumptions for the purpose of this study.

We consider performing patrolling task in a continuous and homogeneous domain. Every point of a homogeneous domain has the same priority to be patrolled (this characteristic has been referred to as identical domain in some studies). Moreover, the robots are able to move along a homogeneous domain using their maximum speed without the need to change their speed (this characteristic has been refereed to as even (uniform) domain in some studies). We also restrict ourselves to a static domain, where there will be no change neither in the state (homogeneous or heterogeneous), nor in the configuration of the region within the execution of the patrolling algorithms. For instance, moving obstacles, reports of intruders penetration, or falling debris may respectively change the configuration, priority of the points, and uniformity of the region to be patrolled. We may alternatively refer to the domain as region, train, environment, or work area. Furthermore, without the loss of generality, we may consider an open curve (i.e., open fence) as a segment, and a close curve (i.e., closed fence) as a circle (cycle).

We also consider a weak model for the robots in order to make our results as general as possible. We add capabilities only as needed for a specific problem, or in order to examine how these added capabilities improve the patrolling performance. Therefore, unless specified otherwise, each robot will have the following capabilities and characteristics: 
Mobility. Each robot is able to move at its own maximum speed.

Oblivious. Robots have no ability to remember any information about their previous states, or the environment.

Silent. Robots do not have the ability to communicate with each other.

Blind. Robots do not have the ability to see their neighborhood. We may alternatively refer to blindness as zero visibility or point-visibility.

Synchronized. Robots have synchronized clocks in order to execute algorithms synchronously.

Reliable. Robots never send false reports, or fail to perform their patrolling tasks. We may alternatively refer to robots as agents or patrolmen.

Finally, as the performance criteria, we consider the worse idle time, denoted by $(I)$, which is the maximum time when a point of the region remains unvisited by any robot.

\subsection{Our Contribution}

In this section, we give a brief overview of the patrolling problems addressed in the current study, and the results obtained. The detailed and formal problem statements, as well as the results are presented in the subsequent chapters.

Patrolling Graphs by Faulty Mobile Robots. We study the patrolling problem when some of the patrolmen may be unreliable (faulty) such that they do not report their monitoring activities. More specifically, we model and study the following problem: we are given a team of $k$ patrolmen, and a domain to be monitored. We assume that some of the patrolmen may be unreliable, that is $f$ of $k$ robots are 
faulty and unknown to the rest of team. Our goal is to design a strategy constructing perpetual patrolmen trajectories, so that, no point of the environment will ever be left unvisited by some reliable robots longer than the allowed idle time. This has to be independent of which subset of the robots (of a given size) will turn out to be faulty. We give a fault-tolerant patrolling strategy for open fences (segments), which is optimal when $f$ is odd, and near optimal when $f$ is even. We also provide an optimal patrolling strategy for Eulerian graphs. Finally, we prove that finding an optimal patrolling strategy for some general graphs is NP-hard.

Patrolling Graphs by Mobile Robots Equipped with Visibility. We consider a setting where a team of $k$ mobile robots have to patrol a given region assuming that each mobile robot is able to observe some neighborhood of its current position. For this purpose, robots move perpetually around the region in order to see each point of the region as often as possible. We propose optimal algorithms for the robots patrolling a fence (whether open or close) where all robots have the same maximum speed, but are equipped with distinct visibility ranges. We then briefly study the case of distinct speeds and visibility ranges, thus showing that the fence (whether close or open) patrolling is entirely different for the robots with distinct visibility ranges than the case of point-visibility robots. Further, we show that for general graphs, the patrolling problem by robots with the same speed and distinct visibility ranges is NP-hard; this contrasts sharply with the patrolling problem by point-visibility robots which has been known to have a polynomial-time solution (see [35]). 
Patrolling Trees by Homogeneous Mobile Robots. Patrolling by a team of homogeneous mobile robots, where all agents have the same speed and visibility may seem straightforward. However, as far as we know, no optimal patrolling strategy for general graphs has been proved yet. For this purpose, we propose an off-line patrolling schedule, in which placing $k$ robots at specific initial positions on a geometric tree (where robots can change direction and speed anywhere on vertices or interiors of edges), and making them move at unit speed, permits to achieve the optimal idle time. Then, we extend the proposed off-line algorithm to a graph tree model, where the robots can change direction only on vertices. We further consider an on-line patrolling schedule, which behaves according to the so-called rotor-router model. Finally, we will discuss how experimental results indicate that in a random setting, the rotor-router is an efficient patrolling strategy for tree graphs.

\subsection{Thesis Outline}

In Chapter 2, we review a number of patrolling problem models, and provide an overview of the state of the art in the field. In Chapter 3, we introduce our model of faulty robots (unreliable patrolmen), that is in a team of $k$ robots (patrolmen), at most $f$ of them may be unreliable (i.e., they do not report their monitoring

activities). We investigate this problem for various classes of graphs, and propose algorithms to minimize the maximum time between successive visits of every point by a reliable patrolman. In Chapter 4, we study patrolling problem by robots equipped with visibility. We develop the optimal patrolling algorithms for the case of close and open curves, when all robots have the same maximum speed. We 
also briefly discuss the case of distinct speeds. Further, we show that for general graphs, the patrolling problem for robots with the same speed and distinct visibility ranges is NP-hard. In Chapter 5, we look at the settings where the region to be patrolled is a tree. We propose an optimal off-line algorithm for patrolling trees by a team of $k$ mobile robots with the same speed and zero visibility. Then, we analyze the competitive ratio of an on-line patrolling algorithm for a team of $k$ mobile robots following rotor-router strategies. We conduct experiments on a rotor-router simulator to discuss the competitive ratio for randomly generated trees. In Chapter 6, we explain the details of the rotor-router simulator implemented in Java. We then conclude our study in Chapter 7 by summarizing the results and stating some open problems.

\subsection{Publications}

\section{Publications upon which the thesis is based:}

- J. Czyzowicz, E. Kranakis, D. Pajak, N. Taleb. Patrolling by Robots Equipped with Visibility. In proceedings of SIROCCO 2014, July 23 - 25, 2014, Japan (see [31]).

- J. Czyzowicz, L. Gasieniec, A. Kosowski, E. Kranakis, D. Krizanc, N. Taleb. When Patrolmen Become Corrupted: Monitoring a Graph using Faulty Mobile Robots. In proceedings of ISAAC 2015, Dec 09-11, Japan (see [33]).

- J. Czyzowicz, L. Gasieniec, A. Kosowski, E. Kranakis, and N. Taleb. Patrolling Trees with Mobile Robots. To appear. 


\section{Chapter 2}

\section{Related Work}

\subsection{Introduction}

In this chapter, we review some of the most relevant literature on the patrolling problem. We discuss different models of mobile robots, the environment to be patrolled, and the performance criteria. Due to a close connection between the patrolling problem and well-known tasks in computer science, many researchers approached this problem and proposed various patrolling strategies using different techniques.

Patrolling has been studied extensively in the robotics literature. The environment and time considered in robotics models are usually discrete and set in a graph environment. Given such a graph, the patrolling task refers to continuously visiting all the graph nodes so as to minimize the time lag between two visits.

While many authors study heuristic patrolling strategies for various settings and analyze their performance through experiment, patrolling raises interesting theoretical questions. Recent studies have shown that finding an optimal strategy 
is not straightforward, even when the patrolling terrain is as simple as a line segment or circle. The goal in these settings is to visit every point of the environment perpetually.

In this study, we classify patrolling algorithms into four categories as follows; area patrolling, perimeter patrolling, randomized patrolling, and distributed patrolling. In area patrolling, the environment to be patrolled is a two dimensional train, in which every point of the terrain should be visited repeatedly. On the other hand, in perimeter patrolling, only the border of the environment is required to be visited. In a totally different adversarial setting, randomized patrolling is performed in the presence of an adversary who takes advantage of any moment for penetration. Recently, distributed patrolling has attracted numerous attention in which distributed control in patrolling strategies is an important alternative to the centralized settings.

\subsection{Area Patrolling}

In area (two dimensional) patrolling, we are given a polygon enclosing a continuous terrain, and $k$ mobile robots that are required to visit every point repeatedly within the area. In this case, a discrete representation of the environment is typically obtained by creating a robotic road-map (graph), in which the important viewpoints are vertices and the edges represent the links between the vertices. The edges may have different lengths (weights) corresponding to the real distance between the vertices. Another technique to change the representation of the terrain is skeletonization, in which the environment is partitioned into cells such that their sizes 
are the same size as of robot's cover tool.

The next step after creating the robotic graph is to generate trajectories for each of $k$ robots to minimize the time lag between two visits. In general, two approaches can be found in the literature: cyclic strategy, where robots move in one direction around a cycle covering the environment, and partition strategy, in which the environment to be patrolled is partitioned into sections patrolled separately by individual robots (or subsets of robots). Researchers have utilized different methods such as machine learning techniques or graph theory methods to generate robot trajectories.

\section{Machine Learning Methods}

Various machine learning techniques are used to solve the patrolling task. The undertaken approaches vary in different aspects such as agent type and their decisionmaking process, or coordination and communication mechanisms.

Machado et al. [67] first introduced idleness, as a measure of patrolling quality. They described the patrolling problem in terms of movement in a general graph, and the idleness was measured as the average number of time steps between visits to all the vertices. They proposed several multi-agent architectures having variable parameters such as agent type (reactive vs. cognitive), agent communication (allowed vs. forbidden), coordination scheme (central and explicit vs. emergent), agent perception (local vs. global), and decision-making (random selection vs. goal-oriented selection). The conducted experiments showed that agents moving randomly achieved undesirable results. In addition, agents with no communication ability moving towards the nodes with the highest idleness, performed nearly as well as those with the most complex implemented algorithms. 
Machado et al. [66] conducted a more comprehensive study based on [67] (which was discussed in the previous paragraph). They proposed a new evaluation criteria and a more realistic representation of the patrolled terrain. They considered the edge weights as the real distance between the nodes. Their experiments showed that there is no significant change for the majority of agent architectures if the real distance between nodes is considered instead of unitary distance. In other words, the best architectures with unitary distance are still the best architectures considering real distance. The same occurs with the worst architectures. However, there was a noticeable difference in random architectures due to its random behavior.

In previous studies, the effect of interference and refueling has not been taken into account. Sempé and Drogoul [83] have implemented a patrolling approach for robotic agents, in order to address the challenges of the complex issues in the real world, such as battery recharging and physical interference. In their work, patrolling is seen as a task allocation problem, where each robot is assigned a different region to visit. Their proposed collective architecture, is also adaptable to various robot group sizes and environment changes.

Another collective architecture (also adaptable to environment changes) was proposed by Santana et al. [82]. They used reinforcement learning techniques to create adaptive agents that learn how to patrol. Reinforcement learning is often characterized as the problem of learning what to do (i.e, how to map situations to actions) in order to maximize a numerical reward signal [88]. Although not always getting the best results, the adaptive solutions are superior to other solutions as compared in most of the experiments. In addition, their proposed approach is totally distributed, which makes it very efficient as well. 
Ahmadi and Stone [4] proposed an approach where robots use a negotiationbased mechanism to divide an area into sub-areas, where each robot is responsible for the sub-area it is positioned in. Using a different negotiation mechanism Hwang et al. [56] described an approach to patrol infrastructures of all sizes and topologies. In their approach each agent acts as a negotiator and receives a set of random graph vertices to patrol. Agents negotiate those vertices using auctions to exchange them with other agents to minimize visits to the same node.

\section{Graph Theory Methods}

Many researchers used graph theory methods to implement the cyclic or partitioning strategies. Cyclic strategies often rely on either TSP related solutions, or spanning tree based approaches. Different graph theory decomposition methods are used to partition the environment by decomposing the work area into sub-areas.

The theoretical analysis of the patrolling problem was first proposed by Chevaleyre [22]. The author described two graph-theory centralized strategies: cyclic strategies, and partitioning strategies. In cyclic strategies, a TSP cycle is computed and robots are perpetually going around this cycle. In partitioning strategies, the environment is divided to different regions which are assigned to each robot to patrol. Both strategies have generally good performance. The first one is better suited for graphs that are highly connected or have large closed paths. The second one is better when graphs have long corridors separating the regions.

Elmaliach et al. [44] posed patrolling as a visit-frequency optimization problem, and considered patrolling of a non-uniform continuous target area. They divided the area into a grid and created a Hamiltonian cycle (a closed cycle through a graph 
that visits every node of the graph exactly once) in the grid. The robots move along this cycle while maintaining equidistant positions. The velocity of robots may change depending on a given location in the terrain, as well as the direction in which they travel.

Portugal and Rocha [77] presented a multilevel partitioning algorithm that assigns different regions (sub-graphs) to each mobile agent. After partitioning, the algorithm computes paths for every robot using a classical algorithm for Eulerian cycles (a closed path that visits every edge of a graph exactly once), and various heuristics for Hamiltonian cycles, non-Hamiltonian cycles and Longest paths (a simple path of maximum length in the graph). The results show that these algorithms perform better only in half of the cases compared to cyclic algorithm, and obtained worse results in the other half. However, similar to the cyclic algorithm, the partitioning algorithms is deterministic. Nevertheless, it is much more difficult for an intruder to penetrate, because it would need to keep track of the local patrolling path of every single robot.

Pasqualetti et al. [73] and later extended by Pasqualetti et al. [75] proposed a procedure to build a graph (roadmap) to represent the topological structure of the environment to be patrolled. They separately studied patrolling a chain, tree, and cyclic graph. As a performance criteria, they considered refresh time which has the same concept as the idle time.

Pasqualetti et al. [74] considered an environment, in which a set of strategically important locations (viewpoints) with different priorities were defined. The locations with higher priority need to be visited more often. The authors have proposed a patrolling strategy for a team of autonomous agents that move in a 
coordinated way along a constructed tour through the viewpoints.

A comparative study was presented by Almeida et al. [6] (in 2004), which analyzed many different approaches to the patrolling problem. It was observed that the best strategy depends on the topology of the environment and the agents' population size. It was concluded that for most cases the TSP cyclic approach has generally the best performance. However, this architecture has problems in dynamic environments, large graphs and graphs containing long edges, due to their predefined natures. Agents with no communication ability, that move towards the vertex with the highest idleness, performed nearly as well as those when the most complex algorithm implemented. Another survey on multi-robot patrolling algorithms was presented by Portugal and Rocha [78] (in 2011), in which the authors classified the existing patrolling strategies into three categories: pioneer methods, alternative methods, and recent studies.

\subsection{Perimeter Patrolling}

The problem of perimeter (one dimensional) patrolling using mobile robots has many real world applications, and thus has been extensively studied under the names of either boundary patrolling or fence patrolling in the literature. Boundary patrolling usually refers to monitoring the close curve, representing the perimeter of the connected planar region, while fence patrolling corresponds to surveillance of an open curve. In the literature, for simplicity (and without loss of generality), it is usually assumed that the open curve is a line segment and the close curve is a circle. Further, some authors refer to boundary patrolling as patrolling on a circle, and 
fence patrolling as patrolling on a segment. On the other hand, some others refer to fence patrolling as patrolling on either a close or open fence. For the sake of clarity, we explicitly mention open fence patrolling (open curve patrolling, or segment patrolling), and closed fence patrolling (close curve patrolling, or circle patrolling) when needed in this study.

\section{Robots with Equal Speeds}

Elmaliach et al. [42] focused on patrolling along an open fence. They introduced two coordinated techniques: the synchronized and synchronized-overlap methods. The segment in both methods is partitioned into equidistant sub-segments assigned to a robot. In the synchronized technique, each robot perpetually covers its own sub-segment while synchronizing its velocity to its peers, such that they all begin and end sub-segments jointly. In the synchronized-overlap method, robots move in a synchronized way as before (i.e., all robots move to the right/left together), however more than one sub-segment is assigned to each robot, such that the sub-segments overlap (intersect) in space. Each robot enters its neighbors' segments depending on the size of the overlap. In fact, this method is a generalization of the synchronized method, where there is no overlap between the sub-segments. The results show that in general, the synchronized approaches to multi-robot patrolling outperform the unsynchronized methods.

Proposed patrolling strategies by Elmaliach et al. [42], assume that there are no errors in the movement of the robots, no velocity changes, and no uncertainty in the planned trajectories. However, Elmaliach et al. [43] developed a realistic model of robot motion, that considers real-world uncertainties and accumulating motion 
errors. The results of their experiments show that the new model is not only more accurate relative to the previous models, but also accurate on an absolute scale.

Collins et al. [27], considered a heterogeneous one dimensional region, in which only a finite number of boundary segments, called vital regions, need to be patrolled by a set of $k$ mobile robots with the same maximum speed. The remaining part of the boundary, called neutral regions, do not have to be monitored by the mobile robots. However, they may be traversed by the robots since they may be in fact the links to reach the vital regions. The authors have proposed a partition-based strategy to patrol a segment. It is has been proven that the optimal idleness for patrolling the closed curves is always attained by the better of two strategies: the partition strategy, and the cyclic strategy, in which equally-spaced robots patrol the cycle, moving in the same direction. The choice of the strategy depends on the arrangement of the vital regions around the boundary.

\section{Robots with Different Speeds}

Previous studies assume robots have the same maximal speed to patrol the environment. Czyzowicz et al. [30] did the first study of boundary patrolling by agents with distinct maximal speeds. It has been proved that in a homogeneous scenario, where all agents have the same speed, the optimal patrolling strategy for line segment is either the partitioning strategy or cyclic strategy, while only the cyclic strategy achieves the optimal idle time for the circle. However, in a more heterogeneous scenario where robots have different maximal speeds, neither the cyclic strategy nor the partition strategy lead to optimal performance. 
Line Segment. For a line segment, Czyzowicz et al. [30] proposed a proportional partition-based strategy, referred to as algorithm A1. According to this algorithm, each agent moves back and forth perpetually in a segment, the length of which is proportional with its speed. Algorithm $A 1$ has been proved to be optimal when all maximum speeds are equal. This strategy has also been proven to be optimal for any configuration of speeds for $k=2$ agents. Czyzowicz et al. have conjectured, conjecture 1 , that $A 1$ is optimal for any number of robots with arbitrary speeds. However, Kawamura and Kobayashi [58] proved that conjecture 1 holds for $k=3$, but fails in general. They disproved this conjecture by providing two counterexamples involving $k=6$ and $k=9$ agents. Using 6 agents they have proposed an algorithm that achieves an idle time of $c \cdot i d l e(A 1)$ where $c=41 / 42$ and idle $(A 1)$ illustrates the idle time of algorithm $A 1$. The second example that used 9 agents achieves an idle time of $c \cdot i d l e(A 1)$ where $c=99 / 100$.

Dumitrescu et al. [39] improved the ratio $c$ to $(24 / 25+\epsilon)$ for any $\epsilon>0$ by replicating the strategy from the second example and with a number of agents larger than 9. Later, Kawamura and Soejima [59] proposed an algorithm to patrol the segment in $c \cdot i d l e(A 1)$ where $c=3 / 4$. They conjectured that $c=3 / 4$ is the smallest value possible.

Unidirectional Circle. From a theoretical perspective, the closed fence patrolling problem is similar to the classical lonely runners problem, which was introduced independently by Wills [94] and Cusick [28] in number theory and discrete geometry. According to this problem, $k$ agents run clockwise along a circle of length 1 , starting from the same point at time $t=0$. They have distinct but constant speeds. A runner is called lonely when he/she is at distance of at least $1 / k$ from any other 
runner (along the circle). The conjecture asserts that each runner is lonely at some time. The conjecture has only been confirmed for up to $k=7$ runners (cf. [14, 15]).

Recently, Czyzowicz et al. [30] extended the runner problems to circle patrolling by mobile robots with distinct speeds. They proposed algorithm $A 2$ for the variant speeds, in which all agents are required to move in the same direction along a circle (say counterclockwise). This algorithm only uses a subset $r$ of agents having sufficiently high maximal speeds. These agents are placed on the circle at even distances, and move counterclockwise using the maximal speed of the slowest agent from the subset. The value of $r$ is chosen such that the idle time is minimized. Algorithm $A 2$ has been proved to be optimal for all configurations of speeds with $k<5$ agents, under the additional constraint that all the agents are restricted to motion in the same direction around the boundary. Czyzowicz et al. [30] conjectured, conjecture 2, that algorithm $A 2$ is optimal for any number of agents patrolling the circle. However, Dumitrescu et al. [39] disproved this conjecture by proposing a counterexample for $k=32$ agents. They constructed a schedule for 32 agents with harmonic speeds, i.e., $v_{i}=1 / i, i=1, \ldots, 32$, that has an idle time strictly less than idle(A2). In another study, Kawamura and Soejima [59] conjectured that algorithm $A 2$ does not have a constant approximation ratio. They demonstrated that, for any constant $c$, a schedule exists that patrols a circle with less idle time than idle(A2).

Bidirectional Circle. Czyzowicz et al. [30] have also considered patrolling of a circle which may be traversed in both, clockwise and counterclockwise directions. It has been proved that the cyclic strategy (proposed for the unidirectional circle) is also optimal for $k=2$, for agents that can change direction of motion. However, it has been shown that it is no longer optimal for $k=3$ agents, and a new type 
of strategy which is neither partition-based nor cyclic achieves a shorter idle time. Dumitrescu et al. [39] proposed a new algorithm, algorithm $A 3$, that outperforms both $A 1$ and $A 2$ for some speed settings for all $k>3$ agents patrolling a bidirectional circle.

Point Patrolling. A new problem called point patrolling problem was introduced by Kawamura and Soejima [59]. In fact, this problem is a simplification of the segment patrolling problem, in which agents patrol a single point instead of a segment. It turns out that this problem can be reduced to a discrete decision problem, where given time $t$, you need to decide whether the idle time can be at most $t$ or not. It has been shown that the generalized point patrolling problem is NPcomplete.

\subsection{Randomized Patrolling}

The problem of patrolling an area with existence of an adversary is applicable in many security applications. In these adversarial settings, a possible disadvantage of deterministic algorithms is that an intelligent adversary that comprehends the patrolling strategy may take advantage of idle time between passages of robots in some points of the area and easily penetrate. On the other hand, if robots move non-deterministically, the choice of penetration position becomes less trivial.

Sak et al. [81] proposed various strategies to partition the environment among robots, and to compute trajectories for robot motion. They evaluated their strategies against three types of intruders: a random intruder, an intruder that waits until the robot leaves the site to initiate the attack, and an intruder that uses statistics 
to forecast the time of the next visit to the site. They presented the best strategies for each of these scenarios, and evaluated them using three metrics which approximate the probability of penetration for each type of intruder.

A stronger adversarial model was considered by Agmon et al. [2]. In their settings, the adversary knows the patrolling strategy of robots, and therefore will choose to penetrate where it has the lowest probability of being detected. They have described a polynomial time algorithm to maximize the probability of penetration detection.

Amigoni et al. [8] considered a model of adversarial settings in a game theory framework. They have defined a game in which two players, i.e., a patroller and an intruder, take sequential actions (like in chess). They proposed algorithms to find optimal strategies to maximize the expected utility of robotic patroller.

In a similar study, Alpern et al. [7] presented a class of patrolling games, in which the attacker (intruder) needs some consecutive periods, uninterrupted by the patroller, to penetrate to the environment and win. In this scenario, patrolling is in fact a win-lose game. Given best play on both sides, they defined a value which is the probability that the patroller wins. For various classes of graphs, they analytically determined either the value of the game, or bounds on the value.

\subsection{Distributed Patrolling}

Distributed (decentralized) patrolling is an important alternative to centralized patrolling, in which a centralized controller leads agents to carry out a mission. In 
distributed settings, on the other hand, the agents achieve a goal by communicating with each other in different ways, for example, sending messages, leaving tokens, etc. In one distributed approach, Czyzowicz et al. [34] showed convergence of a dynamic system of $k$ robots which communicate by merely bouncing against each other. Recently, Pasqualetti et al. [76] studied the distributed coordination of a set of moving cameras monitoring a line segment to detect moving intruders. Other distributed patrolling strategies, based on swarm or ant-based algorithms were introduced in $[45,68]$. In these approaches, agents are supposed to be memoryless (or having finite memory), and with local sensing capabilities. Ant-like algorithms usually mark visited nodes of graph to minimize visits to the same nodes.

One of the most popular ant-based approach is rotor-router [91], a distributed model with local control at nodes for managing movement of robots in a graph. In this model, a group of identical robots are initially placed at nodes of a graph (initial locations of robots), where each node of the graph maintains a cyclic ordering (considered as ports' pointers) of outgoing edges. During consecutive turns, robots visiting a node are propagated along outgoing edges pointed by exit port, according to ports' pointers in a round-robin fashion. The behavior of the rotor-router model is fully deterministic. All relevant rotor-router concepts mentioned in this section will be precisely defined in Chapter 5 .

The first study of the patrolling problem based on rotor-router model was introduced by Yanovski et al. [96]. They showed that a single agent following rotorrouter approach enters, after a transient period, an Eulerian cycle during which all edges are traversed an identical number of times. They proved that independently 
of the initial configuration (defined by the initial locations of robots, ports' pointers of every node, and initial exit port at each node) of rotor-router mechanism, the agent gets into an Eulerian cycle in $O(2 m D)$ time steps, where $m$ and $D$ are number of edges and diameter of the graph receptively. Bampas et al. [12] examined in more details the dependence of lock-in time (stabilization time) ,i.e., the number of time steps until the robot gets into an Eulerian cycle, on initial configuration. They performed a case study in the form of a game between a player intending to lockin the agent into Euler tour as quickly as possible, and an adversary with a counter objective. They observed that in certain (easy) cases the lock-in can be achieved in $O(m)$ time steps. On the other hand, if the adversary is solely responsible for the assignment of ports pointer and initial exit ports, the $\Omega(m D)$ lock-in time can be enforced in any graph.

In terms of cover time, which is the number of time steps until every node of the graph has been visited by at least one robot, Yanovski et al. [96] and Bampas et al. [12] showed that a single agent achieves a cover time of exactly $\Theta(m D)$ time steps for any graph with $m$ edges and diameter $D$ (Yanovski et al. [96] showed the upper bound, and Bampas et al. [12] showed the lower bound). Later, Friedrich and Sauerwald [48] investigated the cover time of a single agent for specific graph classes.

In a different study, the robustness properties of a single rotor-router were considered by Bampas et al. [13]. They investigated the time required for a rotor-router system to stabilize to a (new) Eulerian cycle in the presence of faults in the exit ports, or dynamical changes in the graph, that is adding or removing edges.

The case of multiple parallel rotor-routers, was first studied experimentally by 
Yanovski et al. [96]. They made a conjecture that a system of $k>1$ parallel routers stabilizes, after at most $2(1+1 / k) m D$ time steps, with a period of length at most $2 m$ time steps. Later, Chalopin et al. [21] disproved this conjecture, showing that the periodicity of parallel rotor-routers can in fact, be super polynomial in size of graph. They provided polynomial upper bounds of $O\left(m^{4} D^{2}+m D \log k\right)$ and $O\left(m^{5} k^{2}\right)$ on number of time steps it takes for the system to stabilize. Further, they discussed a new concept of sub-cycle decompositions which plays an important role in understanding the periodicity of rotor-router patrolling strategy.

Cover time of multiple parallel routers was first considered by Klasing et al. [61]. They showed that for a special case when the graph is a cycle, a $k>1$ parallel system explores a cycle $\Theta(\log k)$ times faster than a single agent. Later, Dereniowski et al. [36] provided tight bounds on cover time of $k$ parallel routers in a graph. They showed that for any graph, the cover time of rotor-router is at most $O(m D / \log k)$, and at least $\Omega(m D / k)$ time steps, which receptively corresponds to speedup of $\Theta(\log k)$ and $\Theta(k)$ with respect to a single rotor-router system. In a similar study, Kosowski and Pajak [64] determined the precise asymptotic value of cover time for $k$ parallel routers for degree-restricted expanders, random graphs, and constant-dimensional tori.

\subsection{Conclusion}

In this chapter, we reviewed some of the most relevant studies to the patrolling problem. We classified the existing patrolling algorithms based on the environment to be patrolled, and the strategies used to solve the problem. In the perimeter 
(one dimensional) patrolling only the boundary of the environment is required to be patrolled. On the other hand, in the area (two dimensional) patrolling, every point of a two dimensional environment is supposed to be patrolled. In an adversarial setting, randomized patrolling is performed in the presence of an intruder attempting to penetrate to the environment. Unlike centralized strategies, in distributed (decentralized) patrolling the robots perform the patrolling task in the absence of a central controller. 


\section{Chapter 3}

\section{Patrolling Graphs by Faulty Mobile}

\section{Robots}

\subsection{Introduction}

In this chapter, we are interested in patrolling when some of the patrolmen may be unreliable (faulty) such that they do not report their monitoring activities. Considering a team of $k$ robots, where at most $f$ of them may be unreliable, what algorithm should be followed so as to minimize the maximum time between successive visits of every point by a reliable patrolmen? We denote by $I_{k}^{f}(G)$ the maximum idle time that a point of the domain may remain unvisited by reliable patrolmen. The objective is to find patrolling strategies minimizing the idle time $I_{k}^{f}(G)$.

We investigate the problem of patrolling by unreliable robots for various classes of graphs. We design optimal algorithms for line segments, which turn out to be surprisingly different from strategies proposed for related patrolling problems in the literature (when $f=0$, either the cyclic or partitioning strategy achieve the optimal idle time for the line segments). We then use these results to study the 
case of general graphs. For an Eulerian graph $G$, which is a graph where every vertex has even degree, we show the optimality of cyclic strategy for patrolling by unreliable robots (when $f=0$ the cyclic strategy achieves the optimal idle time for Eulerian graph as well). Further, we prove that computing optimal idle time for arbitrary graphs is NP-hard (when $f=0$, the cyclic strategy achieves the idle time for arbitrary graphs in polynomial time). We show the hardness of the problem of computing the optimal idle time for three robots, at most one of which is faulty, by reduction from 3-edge-coloring of cubic graphs - a known NP-hard problem . A byproduct of our proof is the investigation of classes of graphs minimizing the idle time (with respect to the total length of edges); an example of such a class is known in the literature under the name of Kotzig graphs.

\subsubsection{Preliminaries and Notation}

We are given a connected topological graph $G=(V, E)$ with $V$ being its set of vertices and $E$ its set of edges. In the sequel we define several useful concepts.

The Jordan arc representing each edge $e \in E$ of the graph $G=(V, E)$ is modeled as a smooth continuous and rectifiable curve of arbitrary positive length represented by its edge weight $w(e)$. We may suppose that the graph is embedded in $3 D$ space, with no edge crossings. By $|E|$ we denote the sum of the lengths of the edges of $G$.

At any time a robot may occupy any point belonging to edge $e$ (so the sum of its distances from both endpoints of $e$ sums up to $w(e))$. We denote by $\mathcal{D}_{G}$ the domain (the union of edges) along which the robots walk. We assume a continuous traversal model, whereby the movement of the $i$-th robot within $\mathcal{D}_{G}$ follows a 
continuous function of time $\pi_{i}:[0, \infty) \rightarrow \mathcal{D}_{G}$, for each $i=1,2, \ldots, k$. Hence, $\pi_{i}(t)$ denotes the position in $\mathcal{D}_{G}$ of the $i$-th robot at time $t$. Each robot may move in any direction along an edge not exceeding the maximum (unit) speed so within time interval $\left[t_{1}, t_{2}\right]$ each robot may travel a distance of at most $t_{2}-t_{1}$. We also suppose that when walking at maximum speed, a robot travels the unit distance in unit time, so that time and distance traveled are commensurable. By patrolling strategy we understand the set $\mathcal{P}=\left\{\pi_{1}, \pi_{2}, \ldots, \pi_{k}\right\}$ of infinite trajectories of $k$ robots in $\mathcal{D}_{G}$, where $\pi_{i}(t)$ is the point of $\mathcal{D}_{G}$ occupied by the $i$-th robot at time $t$.

The performance of the patrolling strategy is evaluated by using a measure of idleness. Suppose that we design the patrolling strategy $\mathcal{P}=\left\{\pi_{1}, \pi_{2}, \ldots, \pi_{k}\right\}$ for $k$ robots moving in the domain of a geometric graph $G$ when each robot is reliable. Then the idleness of strategy $\mathcal{P}$ for graph $G$ (or its idle time), denoted by $I_{k}^{f}(G, \mathcal{P})$ is the supremum of the lengths of time intervals between two consecutive visits to the same point of $\mathcal{D}_{G}$ (supremum taken over time and all points of $\mathcal{D}_{G}$ ). When up to $f$ robots may be faulty, we assume that the adversary, knowing our strategy, may choose a set $F$ of $f$ faulty robots, a point $p$ of the domain and a time moment $t \geq 0$. The idleness of the strategy is the supremum (taken over all such adversarial choices) of time intervals $T$ such that point $p$ is not visited during the time interval $[t, t+T]$ by any reliable robot. Finally, the idleness of a graph $G$ for $k$ robots, at most $f$ of which may be faulty, is denoted by $I_{k}^{f}(G):=\inf _{\mathcal{P}} I_{k}^{f}(G, \mathcal{P})$. Hence $I_{k}^{f}(G)$ is the lower bound of idleness over all possible patrolling strategies. When there are no faulty robots (i.e., $f=0$ ) we use the notation $I(G, \mathcal{P}):=I_{k}^{0}(G, \mathcal{P})$.

Consider a walk of a robot within the segment, which starts at one of its endpoints, walks to the other endpoint and returns to the initial one. Such a cyclic 
path around the segment has length equal to twice its size. By an Eulerian tour of the segment by $k$ robots we mean a perpetual movement of these robots, which are equally spaced around such a cyclic path, and walking in the same cyclic direction with the same speed. By $C P T(G)$ we denote the length of a Chinese Postman Tour on the graph $G$, which is the shortest closed path that visits every edge of $G$.

\subsubsection{Outline and Results}

In Section 3.2, we provide optimal patrolling strategies for line segments. These non-intuitive strategies rely on a decomposition of the set of robots into three groups with different patrolling tours, in a way dependent on $k$ and $f$. Next we employ these results in Section 3.3 as building blocks to provide strategies for general graphs. In particular, for any Eulerian graph $G$ we show that the idleness satisfies $I_{k}^{f}(G)=(f+1)|E| / k$. In Section 3.3.2, we analyze the hardness of the problem of computing the idle time on a specific class of graphs (derived from the class of Kotzig graphs) by showing that if the idle time could be computed optimally then we could solve 3-edge-coloring of cubic graphs, a well known NP-hard problem (see [49]). Finally, in Section 3.4 we conclude with a summary of our results and mention additional work and various related open problems.

\subsection{Open Fence Patrolling}

In this section we study exclusively the idleness of the line segment and provide upper and lower bounds for idleness. Without loss of generality we assume that we have to patrol the unit-length segment, represented by the interval $S=[0,1]$. 
However, the results can be easily reformulated for segments of any given length. Throughout the main part of this section we assume that most of the robots are reliable, more precisely that $f<\frac{k-2}{2}$. We first give a patrolling strategy and analyze its performance. Then we analyze the lower bound for segment idleness showing that our strategy is optimal for odd $f$ and almost optimal for even $f$.

\subsubsection{The Upper Bound}

The idea of the strategy is the following. We partition the segment $S$ into three subsegments $S_{L}$ (left), $S_{R}$ (right), and $S_{M}$ (middle), where $S_{M}$ does not contain any

endpoint of $S$. Two subsets of robots will follow Eulerian tours of $S_{L}$ and $S_{R}$ and the remaining robots are assigned to do the Eulerian tour of the entire $S$. We show that by choosing the sizes of the segments of the partition as well as the number of robots assigned to each Eulerian tour we obtain an efficient strategy. We have the following theorem:

Theorem 3.2.1 Consider $k$ robots patrolling segment $S=[0,1]$, with at most $f$ of them faulty where $k>2$ and $f<\frac{k}{2}-1$. There exists a patrolling strategy $\mathcal{P}$ of $S$ whose idleness satisfies $I_{k}^{f}(S, \mathcal{P}) \leq \frac{2 \mid f / 2]+2}{k-2[f / 2 \mid}$.

Proof. First we give explicitly the patrolling strategy.

1. Decompose the unit interval $S$ into three segments $S_{L}, S_{M}$ and $S_{R}$ with pairwise disjoint interiors:

$$
S_{L}:=\left[0, \frac{[f / 2]}{k-2[f / 2]}\right], \quad S_{M}:=\left[\frac{\lceil f / 2]}{k-2[f / 2]}, 1-\frac{\lceil f / 2]}{k-2[f / 2]}\right], \quad S_{R}:=\left[1-\frac{\lceil f / 2]}{k-2[f / 2]}, 1\right]
$$


2. For each of the segments $S_{L}, S_{R}$ assign $\lceil f / 2\rceil$ equally spaced robots to perform an Eulerian tour of this segment.

3. The remaining $k-2\lceil f / 2\rceil$ robots perform an Eulerian tour of the entire segment $S$. These robots are also equally spaced around $S$.

Observe first that the subsegments $S_{L}, S_{R}, S_{M}$ are well defined. Indeed, as $f$ is an integer $f<\frac{k}{2}-1$ implies $f \leq \frac{k-3}{2}$. Hence $2\lceil f / 2\rceil \leq f+1 \leq \frac{k-3}{2}+1=\frac{k-1}{2}$. However, for any integer $k>2$ we have $\frac{k-1}{2} \leq k-2$ which implies $2\lceil f / 2\rceil \leq k-2$, so the denominator of the fractions in the definitions of the segments $S_{L}, S_{M}, S_{R}$ is not zero. Moreover, the point $1 / 2$ belongs to $S_{M}$, hence all these segments are well defined.

Denote by $A_{L}$ (respectively $A_{R}, A_{S}$ ) the set of robots executing an Eulerian tour of $S_{L}$ (respectively $S_{R}, S$ ). Observe that the distance $d$ between two consecutive robots of $A_{S}$, computed around this Eulerian tour, equals $d=\frac{2}{k-2[f / 2]}$. We now prove the correctness of the upper bound on the idleness of any point $p \in S$. We consider two cases: when $p$ belongs to an extremal subsegment $S_{L}$ or $S_{R}$ and when $p$ is in the middle segment $S_{M}$.

Case 1: Point $p$ is in an extremal subsegment (by symmetry we may assume without loss of generality that $p \in S_{L}$ ). Suppose first that at least one robot $a_{i} \in A_{L}$ is not faulty. Then $a_{i}$ revisits every point of $S_{L}$ at time intervals of at most $2\left|S_{L}\right|$. Hence the idleness of $p \in S_{L}$ (maximized at endpoints of $S_{L}$ ) is bounded by

$$
I_{k}^{f}(S, \mathcal{P}) \leq 2\left|S_{L}\right|=\frac{2\lceil f / 2\rceil}{k-2\lceil f / 2\rceil}<\frac{2\lfloor f / 2\rfloor+2}{k-2\lceil f / 2\rceil}
$$

When all $\lceil f / 2\rceil$ robots of $A_{L}$ are faulty, the idle time is maximized for $p=0$, while 
the adversary chooses the remaining $\lfloor f / 2\rfloor$ faulty robots to form a stream of consecutive robots of $A_{S}$. Then the time between visits of point $p=0$ by two reliable robots (i.e. one preceding and one following such a stream) equals $d(\lfloor f / 2\rfloor+1)$ and we have

$$
I_{k}^{f}(S, \mathcal{P}) \leq d(\lfloor f / 2\rfloor+1)=\frac{2(\lfloor f / 2\rfloor+1)}{k-2\lceil f / 2\rceil}
$$

again verifying the claim of the theorem. The argument is entirely symmetric when $p \in S_{R}$ and is therefore omitted.

Case 2: $p \in S_{M}$. The visits to this point are made exclusively by the robots from $A_{S}$. Point $p$ is being visited by two streams of robots executing the Eulerian tour of $S$, one walking over $p$ from left to right and the other one from right to left (clearly, in the Eulerian cycle the robots are moving in the same direction, but from the "point of view of the point $p$ " the traversal is in opposite directions). Each of these streams may have several faulty robots, and the idle time at $p$ depends on the distance between the two reliable robots starting and ending such faulty streams.

Consider first the case when the two faulty-robots streams, visiting $p$ at the same time, are disjoint, i.e. separated by at least one reliable robot (cf. Figure 3.1 (a), where $\circ$ denotes a reliable robot and $\bullet$ a faulty robot).

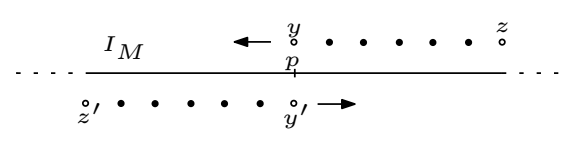

(a)

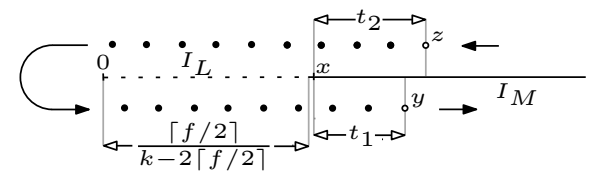

(b)

FIGURE 3.1: In the Eulerian cycle the robots are moving in the same direction, but from the "point of view of the point $p$ " the traversal is in opposite directions 
To maximize the time while point $p$ remains unvisited by reliable robots, the adversary has to make faulty two sequences of consecutive robots (i.e. those belonging to both left-to-right and right-to-left streams) arriving at $p$ at the same time. The idle time is then determined by the length of the shorter of the two sequences of consecutive faulty robots, which in the worst-case contains $\lfloor f / 2\rfloor$ robots. Then the claim of the theorem is again satisfied by Equation (3.1).

Consider now the case when there is a single faulty-robot stream visiting $p$ in both directions (see Figure 3.1 (b) which depicts a time moment $t$ when this happens). In the worst case this stream may contain $f$ robots. Let $t_{1}$ be the time since the last visit of $p$ by a reliable robot $y$ and $t_{2}$ - the time when the next reliable robot $z$ visits $p$. As $x>\left|S_{L}\right|=\frac{\lfloor f / 2\rfloor}{k-2 \mid f / 2\rceil}$ and all robots present within $S_{L}$ at time $t$ are faulty, as well as the distance between $y$ and $z$ around the Eulerian cycle is at most $d(f+1)$, we have

$$
I_{k}^{f}(S, \mathcal{P})=t_{1}+t_{2} \leq d(f+1)-2\left|S_{L}\right|=\frac{2(f+1)}{k-2\lceil f / 2\rceil}-\frac{2\lceil f / 2\rceil}{k-2\lceil f / 2\rceil}=\frac{2\lfloor f / 2\rfloor+2}{k-2\lceil f / 2\rceil} .
$$

This completes the proof of Theorem 3.2.1.

\subsubsection{The Lower Bound}

We first show the following lemma, which applies to general graphs.

Lemma 3.2.2 Consider a patrolling strategy $\mathcal{P}$ of graph $G$. Let $E^{\prime}$ be a subset of segments of edges of $G$, such that starting from some time moment of the strategy, in the union of the interiors of all elements of $E^{\prime}$ there are always at most $n$ robots. Then $I_{k}^{f}(\mathcal{S}, \mathcal{P}) \geq \frac{(f+1)\left|E^{\prime}\right|}{n}$, where $\left|E^{\prime}\right|$ denotes the sum of lengths of segments of $E^{\prime}$. 
Proof. Fix any time interval of length $I^{*}=I_{k}^{f}(\mathcal{S}, \mathcal{P})$ and consider the walk of robots during this interval. The sum $\sigma$ of lengths of trajectories of $n$ robots within $E^{\prime}$ is at most $n I^{*}$. Consider first the case when $\sigma<(f+1)\left|E^{\prime}\right|$. Then there exists some point $p$ of $E^{\prime}$ which is visited by at most $f$ robots. Consequently, the adversary can make all these robots faulty and point $p$ is not visited during a time interval of length $I^{*}$ by any reliable robot, proving the claim of the lemma. In the remaining case we have $\sigma \geq(f+1)\left|E^{\prime}\right|$. Since $\sigma \leq n I^{*}$ we have $I^{*} \geq \frac{(f+1)\left|E^{\prime}\right|}{n}$. This proves Lemma 3.2.2.

The next theorem proves that the patrolling strategy from the previous section is optimal for odd $f$ and almost optimal for even $f$.

Theorem 3.2.3 For any $k$ and $f$ such that $f<k / 2-1$ we have $I_{k}^{f}(\mathcal{S}) \geq \frac{f+1}{k-f-1}$.

Proof. Partition the unit interval into the following three segments

$$
S_{L}:=\left[0, \frac{f+1}{2(k-f-1)}\right], \quad S_{M}:=\left(\frac{f+1}{2(k-f-1)}, 1-\frac{f+1}{2(k-f-1)}\right), \quad S_{R}:=\left[1-\frac{f+1}{2(k-f-1)}, 1\right]
$$

By the condition $f<k / 2-1$ in the hypothesis of the theorem the three subsegments should not have a non-trivial overlap. Before proving the theorem we derive a crucial claim.

Claim. If $I_{k}^{f}(\mathcal{S})<\frac{f+1}{k-f-1}$ then at each time moment during the patrolling there must be at least $f+1$ robots in each of the segments $S_{L}$ and $S_{R}$.

By symmetry it is sufficient to prove this claim for the segment $S_{L}$. Suppose that $I_{k}^{f}(\mathcal{S})<\frac{f+1}{k-f-1}$ and assume on the contrary that at some time, say $t_{0}$, we have at most $f$ robots in the segment $S_{L}$. If an adversary makes all of these robots faulty then it would follow that no reliable robot could visit the endpoint 0 during the 
entire time interval $\left[t_{0}-\left|S_{L}\right|, t_{0}+\left|S_{L}\right|\right]$, where $\left|S_{L}\right|$ denotes the length of the interval $S_{L}$. Therefore the idle time at the endpoint 0 would be larger than $2\left|S_{L}\right|=\frac{f+1}{k-f-1}$, which contradicts the hypothesis of the claim.

From the Claim above we see that at all times each of the two intervals $S_{L}$ and $S_{R}$ contains at least $f+1$ robots. Since at each time moment at least $2 f+2$ robots must be visiting $S_{L}, S_{R}$, the open interval $S_{M}$ must always contain at most $k-2 f-2$ robots. Applying Lemma 3.2.2 to the set $E^{\prime}$ consisting of the segment $S_{M}$, since $\left|S_{M}\right|=\left(1-\frac{f+1}{k-f-1}\right)$ we have

$$
I_{k}^{f}(\mathcal{S}) \geq \frac{(f+1)\left|S_{M}\right|}{(k-2 f-2)}=\frac{(f+1)\left(1-\frac{f+1}{k-f-1}\right)}{(k-2 f-2)}=\frac{f+1}{k-f-1} .
$$

\subsection{Arbitrary Graphs Patrolling}

In this section we study upper and lower bounds for patrolling times on general graphs. First we prove a theorem associating the patrolling time to the length of a Chinese Postman Tour (CPT) on the graph. Next we use the results in Section 3.2 concerning line segments so as to determine asymptotic bounds on the patrolling time for arbitrary graphs. The efficiency of the proposed strategy is arbitrarily close to the optimal one when $k$ is sufficiently large. 


\subsubsection{A General Result and Algorithm}

First we prove the following theorem and approximation patrolling strategy on arbitrary graphs.

Theorem 3.3.1 For any connected graph $G$, and $k \geq 2$ robots, at most $f$ of which are faulty, $(f \leq k-1)$, we have that

$$
\frac{(f+1)|E|}{k} \leq I_{k}^{f}(G) \leq \frac{(f+1) C P T(G)}{k} .
$$

Proof. The upper bound is implied from the following patrolling algorithm:

1. Select any Chinese Postman Tour of $G$.

2. Have the robots patrol the graph by placing them equidistant along the Chinese Postman Tour.

It is clear that the respective distances between consecutive robots will be $\frac{C P T(G)}{k}$. The worst case idle time occurs when we have $f$ consecutive faulty robots. In this case the resulting idle time will never exceed $\frac{(f+1) C P T(G)}{k}$, which proves the upper bound.

The lower bound follows directly from Lemma 3.2.2 applied to $E^{\prime}$ being the set of all edges of $G$.

As a corollary of Theorem 3.3.1 we obtain the following tight (and simple) expression for the value of the idleness for Eulerian graphs.

Corollary 3.3.2 For any connected Eulerian graph $G$, and $k \geq 2$ robots, at most $f$ of which are faulty $(f \leq k-1)$, we have that $I_{k}^{f}(G)=\frac{(f+1)|E|}{k}$.

The claim is immediate since in this case $|E|=C P T(G)$. 


\subsubsection{Hardness of Computing the Idleness}

To show the hardness of our problem in general graphs, we restrict ourselves to the special case of $k=3$ robots with exactly $f=1$ fault. We will now prove that the problem of computing the idleness $I_{3}^{1}(G)$ is NP-hard for general graphs with unit-length edges. The proof proceeds by reduction from 3-Edge-Coloring in Cubic Graphs (3ECC), a well-known NP-complete problem (see [55]).

First we show the following auxiliary result which partially characterizes graphs having minimum possible idleness $I_{3}^{1}$ with respect to the total length of their edges. For a graph $H=(V, E)$ and $E^{\prime} \subseteq E$, denote by $H\left[E^{\prime}\right] \subseteq H$ the connected subgraph of $H$ with edge set $E^{\prime}$.

Lemma 3.3.3 Let $H=(V, E)$ be a graph with unit-length edges.

(i) If $I_{3}^{1}(H)=\frac{2}{3} E(H)$, then there exists a partition of the edge set $E=E_{1} \cup E_{2} \cup E_{3}$ such that each of the graphs $H\left[E \backslash E_{i}\right], 1 \leq i \leq 3$, is semi-Eulerian (i.e., connected and with at most two vertices of odd degree).

(ii) Conversely, if there exist a decomposition of the edge set: $E=E_{1} \cup E_{2} \cup E_{3}$, such that $\left|E_{1}\right|=\left|E_{2}\right|=\left|E_{3}\right|=\frac{1}{3} E(H)$ and each of the graphs $H\left[E \backslash E_{i}\right], 1 \leq i \leq 3$, is Eulerian, then $I_{3}^{1}(H)=\frac{2}{3} E(H)$.

Proof. First, note that $I_{3}^{1}(H) \geq \frac{2}{3} E(H)$ by Theorem 3.3.1.

(i) Suppose that $I_{3}^{1}(H)=\frac{2}{3} E(H)$. Consider the time interval $I^{*}=\left[0, \frac{2}{3} E(H)\right)$. For simplicity of notation only, we assume that time 0 is chosen so that at time 0 all three robots are in "general positions", i.e., not located on vertices. Observe that there cannot exist a point on an edge of the graph which was only visited 
by one robot during time interval $I^{*}$. Indeed, suppose that point $x$ was only visited by robot $i, 1 \leq i \leq 3$; then, if robot $i$ turns out to be faulty, point $x$ would be uncovered throughout $I^{*}$, hence $I_{3}^{1}(H)>\frac{2}{3} E(H)$, a contradiction. Now, since the total distance covered by all robots in time interval $I^{*}$ is $2 E(H)$, it follows from the pigeon-hole principle that each point is visited by precisely two distinct robots during interval $I^{*}$, and moreover, no point can be visited by any robot more than once during $I^{*}$. Since a robot which changes direction at a point other than a vertex during $I^{*}$ traverses some point twice, it follows that a robot entering an edge during $I^{*}$ has to continue along this edge until it reaches its other endpoint. Thus, for each robot $i$ there can exist at most two edges for which this robot explored a proper and non-empty subset of points during interval $I^{*}$ : the edge $e_{i}(0)$ on which robot $i$ was located at time 0 , and the edge $e_{i}\left(\frac{2}{3} E(H)\right)$ on which $i$ was located at time $\frac{2}{3} E(H)$.

With every edge $e \in E$ we now associate an integer $c(e) \in\{1,2,3\}$ as follows. If edge $e$ was explored completely by exactly two robots during interval $I^{*}$, then $c(e)$ is defined as the label of the only robot which did not traverse $e$ during interval $I^{*}$. Otherwise, $c(e)$ is set as the label of either of the two robots which did not traverse $e$ in full during interval $I^{*}$. Define $E_{i}=\{e \in E: c(e)=i\}, 1 \leq i \leq 3$. Then, set $E \backslash E_{i}$ contains all edges such that all of their points were traversed in full by robot $i$ during interval $I^{*}$, and does not contain any edges which were not visited at all by robot $i$ during interval $I^{*}$. The edges $e_{i}(0)$ and $e_{i}\left(\frac{2}{3} E(H)\right)$, which may have been traversed in part by robot $i$ during interval $I^{*}$, may either belong to $E_{i}$ or to $E \backslash E_{i}$. Recalling that no point on an edge is visited more than once by robot $i$ during interval $I^{*}$, we obtain that the traversal performed by robot $i$ 
during time interval $I^{*}$, appropriately truncated (or extended) to exclude (or include) the whole of its starting edge $e_{i}(0)$ and final edge $e_{i}\left(\frac{2}{3} E(H)\right)$ determines a semi-Eulerian traversal of graph $H\left[E \backslash E_{i}\right]$. Hence, all of the graphs $H\left[E \backslash E_{i}\right]$ are semi-Eulerian.

(ii) Given a decomposition $E=E_{1} \cup E_{2} \cup E_{3}$ satisfying the assumptions of the claim, we define the route of robot $i$ to be a perpetual traversal of a fixed Eulerian circuit of graph $H\left[E \backslash E_{i}\right]$ at maximal speed. The length of this circuit is $\frac{2}{3} E(H)$. Consider an arbitrary point $x$ on any edge of the graph. The circuits of exactly two robots pass through point $x$. Hence, even if one of these robots is faulty, point $x$ will be visited by the other robot with a time spacing of exactly $\frac{2}{3} E(H)$ between visits. It follows that $I_{3}^{1}(H) \leq \frac{2}{3} E(H)$, which completes the proof of Lemma 3.3.3. We use Lemma 3.3.3 to show the following theorem.

Theorem 3.3.4 It is NP-hard to decide whether for a given graph $H$ with unit-length edges we have $I_{3}^{1}(H)=\frac{2}{3} E(H)$.

Proof. We show that the posed question is NP-hard by reduction from 3-EdgeColoring in Cubic Graphs $(3 E C C)$. Let the $3 E C C$ instance $G=(V, E)$ be a given cubic graph with $V=\left\{v_{1}, \ldots, v_{n}\right\}$. We will define the graph $H=\left(V_{H}, E_{H}\right)$, in which we ask about $I_{3}^{1}$, by taking 7 copies of graph $G$ and inserting auxiliary subgraphs (gadgets) in place of their edges, so as to connect all these copies to one auxiliary vertex $r$ used for garbage collection. Initially, let $V_{H}=V^{(1)} \cup \ldots \cup V^{(7)} \cup\{r\}$, where each $V^{(j)}=\left\{v_{1}^{(j)}, \ldots, v_{n}^{(j)}\right\}, 1 \leq j \leq 7$, is a copy of $V$, and let $E_{H}$ be empty. Next, for each edge $e=\{u, v\} \in E$, for all copies $1 \leq j \leq 7$, we insert in between vertices $u^{(j)}$, $v^{(j)}$, and $r$ of $H$ the gadget $X$ having 21 edges and 12 additional internal vertices, presented in Figure 3.2. We will denote this copy of gadget $X$ by $X^{(j)}(e)$. 


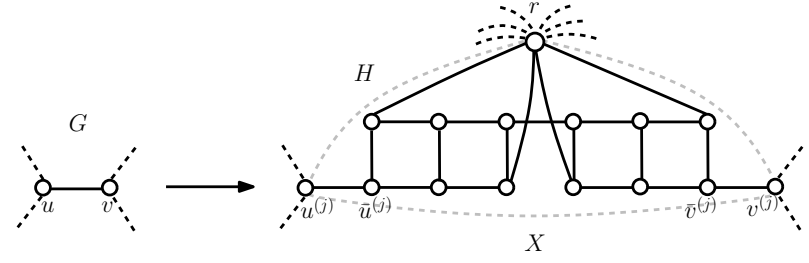

FIGURE 3.2: Gadget $X$ used in the construction of graph $H$ in the hardness proof.

To complete the proof, we now show that $G$ is 3 -edge-colorable if and only if $I_{3}^{1}(H)=\frac{2}{3} E(H)$.

$(\Rightarrow)$ Suppose that $G$ is 3-edge-colorable and fix a coloring of its edges $c: E \rightarrow$ $\{1,2,3\}$. Based on coloring $c$, we define a partition of the edges of $H, E(H)=$ $E_{1} \cup E_{2} \cup E_{3}$, as follows. For each edge $e \in|E|$ having color $c(e)=i$, we consider the 7 corresponding gadgets $X^{(j)}(e)$ in $H$, and for each such gadget assign its edges to sets $E_{1}, E_{2}, E_{3}$ as shown in Figure 3.3.

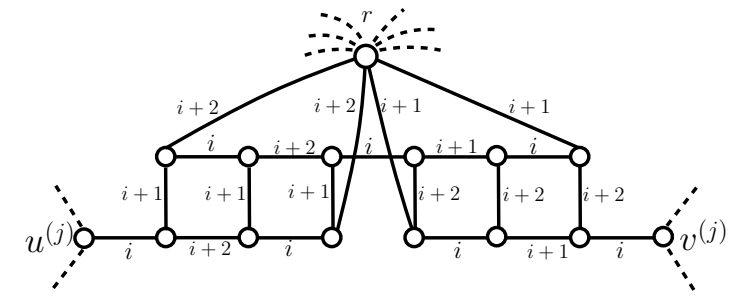

FIGURE 3.3: Assignment of edges of gadget $X^{(j)}(e) \subseteq H$ to sets $E_{1}, E_{2}, E_{3}$ for an edge $e$ having color $i$. Edges marked $i$ in the figure belong to $E_{i}$, edges marked $i+1$ belong to $E_{(i \bmod 3)+1}$, and edges marked $i+2$ belong to $E_{((i+1) \bmod 3)+1}$.

The obtained decomposition $E(H)=E_{1} \cup E_{2} \cup E_{3}$ has the following properties:

- $\left|E_{1}\right|=\left|E_{2}\right|=\left|E_{3}\right|=\frac{1}{3} E(H)$. Indeed, for any gadget $X^{(j)}(e)$, regardless of the color of $e$, we assign 7 edges of the gadget to $E_{1}, 7$ edges to $E_{2}$, and 7 edges to $E_{3}$. Hence, the sets $E_{1}, E_{2}, E_{3}$ have the same number of elements. 
- For $1 \leq i \leq 3$, graph $H\left[E(H) \backslash E_{i}\right]$ has all nodes of even degree. Indeed, for the internal nodes of each gadget $X^{(j)}(e)$, their degree in $H\left[E(H) \backslash E_{i}\right]$ is 2 . Since $c$ is a coloring, each node from $V^{(j)}$ is adjacent to exactly one edge from each of the sets $E_{1}, E_{2}, E_{3}$, hence its degree in $H\left[E(H) \backslash E_{i}\right]$ is also 2. The degree of the last remaining node $r$ must be even as well.

- For $1 \leq i \leq 3$, graph $H\left[E(H) \backslash E_{i}\right]$ is connected. Observe that in each gadget $X^{(j)}(e)$, the subgraph $\left.X^{(j)}(e)\left[E(H) \backslash E_{i}\right)\right]$ is connected and spans all vertices of $X^{(j)}(e)$, including $r$. Since all these gadgets share node $r, H\left[E(H) \backslash E_{i}\right]$ is also connected.

So, we have that $\left|E_{1}\right|=\left|E_{2}\right|=\left|E_{3}\right|=\frac{1}{3} E(H)$ and that graph $H\left[E(H) \backslash E_{i}\right]$ is Eulerian, thus, applying Lemma 3.3.3(ii), we obtain $I_{3}^{1}(H)=\frac{2}{3} E(H)$.

$(\Leftarrow)$ Suppose that $I_{3}^{1}(H)=\frac{2}{3} E(H)$. It follows from Lemma 3.3.3 $(i)$ that there exists a decomposition $E(H)=E_{1} \cup E_{2} \cup E_{3}$ such that each of the graphs $H\left[E(H) \backslash E_{i}\right]$, $1 \leq i \leq 3$, is semi-Eulerian. Let $V_{H}^{(j)}$ be the set of nodes of the connected component of $H \backslash\{r\}$ containing $V^{(j)}, 1 \leq j \leq 7$, and let $H_{+}^{(j)}=H\left[V_{H}^{(j)} \cup\{r\}\right]$. Since $7>3 \cdot 2$, it follows from the pigeon-hole principle that there must exist some $1 \leq j \leq 7$, such that for all $1 \leq i \leq 3$, all vertices from $V_{H}^{(j)}$, have even degree in $H\left[E(H) \backslash E_{i}\right]$. Thus, for the considered value of $j$, each of the graphs $H_{+}^{(j)}\left[E\left(H_{+}^{(j)}\right) \backslash E_{i}\right], 1 \leq i \leq 3$, is Eulerian. Hence, the following properties hold:

- Each vertex $v^{(j)} \in V^{(j)}$ is adjacent in $H_{+}^{(j)}$ to exactly one edge from each of the sets $E_{1}, E_{2}, E_{3}$. Indeed, each vertex $v^{(j)} \in V^{(j)}$ is of degree 3 , and since $H_{+}^{(j)}\left[E\left(H_{+}^{(j)}\right) \backslash E_{i}\right]$ is Eulerian, $v^{(j)}$ may be adjacent to either 1 or 3 edges from $E_{i}$, for all $1 \leq i \leq 3$. It follows that $v^{(j)}$ must be adjacent to exactly one edge from each of these three sets. 
- For each gadget $X^{(j)}(e) \subseteq H_{+}^{(j)}$, where $e=\{u, v\}$, we have that the edge $\left\{u^{(j)}, \bar{u}^{(j)}\right\}$ in this gadget belongs to $E_{i}$ if and only if edge $\left\{\bar{v}^{(j)}, v^{(j)}\right\}$ belongs to the same set $E_{i}, 1 \leq i \leq 3$. This property can be shown for each gadget separately, by exhaustively testing all possible assignments of edges from $X^{(j)}(e)$ to disjoint sets $E_{1}, E_{2}, E_{3}$ so that $H_{+}^{(j)}\left[E\left(H_{+}^{(j)}\right) \backslash E_{i}\right]$ has all nodes of even degree and does not contain small connected components (cycles of 4 or 6 vertices).

We now define a labeling of edges of $G$ with labels $\{1,2,3\}$ as follows. Edge $e=$ $\{u, v\} \in E$ gets label $i$ if in the gadget $X^{(j)}(e) \subseteq H$, edges $\left\{u^{(j)}, \bar{u}^{(j)}\right\}$ and $\left\{\bar{v}^{(j)}, v^{(j)}\right\}$ belong to set $E_{i}$. By the above properties, this assignment yields a well-defined 3-edge-coloring of $G$, which completes the proof.

The above theorem shows that the problem of computing the optimal idle time for patrolling with unreliable robots is NP-hard in general.

\subsubsection{Characterizing Graphs with Minimum Idle Time}

We close this section by considering some properties which hold for graphs with small idle time in fault-tolerant patrolling, and giving examples of classes of such graphs.

For the case of 3 robots, some classes of graphs minimizing idle time $I_{3}^{1}$ (with respect to the total length of edges) are given by Lemma 3.3.3. An example of such a class is known in the literature under the name of Kotzig graphs [65]. A graph is Kotzig if it is 3-regular and admits a decomposition into three matchings $M_{1}, M_{2}, M_{3}$ such that $E=M_{1} \cup M_{2} \cup M_{3}$ and for each pair $i \neq j$, the union $M_{i} \cup$ $M_{j}$ forms a Hamiltonian cycle of the graph. By Lemma 3.3.3(ii), we immediately 
obtain that Kotzig graphs have the minimum possible idleness $I_{3}^{1}$ in the class of cubic graphs, i.e., $I_{3}^{1}=\frac{2}{3} E=\frac{2}{3} \cdot \frac{3}{2} n=n$. Interestingly, this idleness $I_{3}^{1}$ is also best possible in the sense that we cannot obtain better idle time if we know beforehand which of the three robots is faulty, and attempt to solve the problem only for two non-faulty robots: for Kotzig graphs, we have $I_{2}^{0}=\frac{C P T}{2}=\frac{2 n}{2}=n$.

Corollary 3.3.5 $I_{3}^{1}(G)=I_{2}^{0}(G)=n$, for n-vertex Kotzig graphs $G$.

We remark that we do not know of a complete structural characterization of all graphs having minimum idleness $I_{3}^{1}=\frac{2}{3} E$. The characterization from Lemma 3.3.3 is only partial, and the distinction between semi- and Eulerian graphs in claims (i) and (ii) of the Lemma 3.3.3 is important. For example, when the patrolled graph is a cycle with 3 unit-length edges, we have $I_{3}^{1}=\frac{2}{3} E=2$, whereas this graph does not admit a decomposition $E=E_{1} \cup E_{2} \cup E_{3}$ into non-empty sets $E_{1}, E_{2}, E_{3}$ such that each of the graphs induced by $E \backslash E_{i}$ is Eulerian. On the other hand, when the patrolled graph is a star with 3 unit-length edges, this graph admits a decomposition $E=E_{1} \cup E_{2} \cup E_{3}$ into single edges such that each of the graphs induced by $E \backslash E_{i}$ is semi-Eulerian, but this graph does not minimize idle time: by Theorem 3.3.1, $I_{3}^{1} \geq \frac{C P T}{2}=3>\frac{2}{3} E$.

Variants of Lemma 3.3.3 can also be obtained for a larger number of robots. For $k$ even, classes of graphs having minimum possible idle time $I_{k}^{1}=\frac{2}{k} E$ include Hamiltonian Decomposable Graphs, i.e., $k$-regular graphs whose edge set can be partitioned into $k / 2$ edge-disjoint Hamiltonian cycles [72]. 


\subsection{Conclusion and Open Problems}

We gave optimal fault-tolerant patrolling strategy for segments (for odd $f$ ) and Eulerian graphs. In all proposed strategies the collection of patrolmen is divided into sub-collections, each of the sub-collections, forming a cycle of equally spaced robots walking around a portion of the graph (with some portions being covered by more than one sub-collection). Somewhat surprisingly, for a graph as simple as a segment, the optimal strategy consists of two sub-collections patrolling small sub-segments and the third sub-collection patrolling the entire segment (hence the points close to the endpoints being visited by the robots belonging to two subcollections). We also proved that for some graphs finding an optimal patrolling strategy is NP-hard.

While optimal strategies for Eulerian graphs work for any ratio of faulty patrolmen, the strategies for segments assume the maximal faulty robots ratio to be slightly smaller than half the total of all robots. One open question is to give optimal patrolling strategies for segments when the faulty robot ratio is high. There are plenty of open questions concerning different models of patrolling: robot failures may be dynamic, failures may happen with given probability, number of faulty robots $f$ may be unknown, robots may have non-zero visibility radii, or may be allowed to communicate. 


\section{Chapter 4}

\section{Patrolling Graphs by Mobile Robots Equipped with Visibility}

\subsection{Introduction}

In this chapter we study the problem of mobile robots with distinct visibility ranges patrolling a curve. Assume a set of $k$ mobile robots $a_{1}, a_{2}, \ldots, a_{k}$ walking along a unit-length curve in any of the two directions, not exceeding their maximum speeds. Every robot $a_{i}$ has a range of visibility $r_{i}$, representing the distance from its current position at which the robot can see in each direction along the curve. The goal of the patrolling problem is to find the perpetual movement of the robots minimizing the maximum time when a point of the curve (fence) remains unseen by any robot.

When robots have the same speed and zero visibility, the optimal patrolling strategy for open fences is either the partitioning strategy or cyclic strategy, while only the cyclic strategy achieves the optimal idle time for the closed fences ([30]). However, the case of robots with different visibility ranges is more complicated. In 
this chapter, we give the optimal patrolling algorithms for the cases of closed and open fences, when all robots have the same maximal speed. We briefly discuss the case of fence patrolling by robots with distinct speeds and visibility, showing that the boundary patrolling problem for robots with distinct visibility and speed is essentially different than the case of point-visibility robots. However, we give an optimal algorithm for patrolling an open fence by two robots with distinct speeds and visibility ranges. For the case when the environment is a general graph, we show that the patrolling problem for robots with distinct visibility ranges (and the same speed) is NP-hard, while it is known that the same problem for pointvisibility robots has a polynomial-time solution [35].

\subsubsection{Preliminaries and Notation}

Each robot $a_{i}$ is equipped with visibility allowing it to see its environment within its visibility radius $r_{i}$ in both directions from its current position. The visibility ranges of all robots may be different. During the movement of the set of robots, at each time $t$ a point $p$ of the environment is called protected if it is seen by at least one robot. In other words, if robot $a_{i}$ protects point $p$ at time $t$ the distance of the robot $a_{i}$ from $p$ at time $t$ must be at most equal to $r_{i}$. Given a perpetual movement of all robots produced by some patrolling algorithm, by the idle time of point $p$ we mean the smallest value $I(p)$, such that in every time interval $[t, t+I(p)]$ point $p$ is protected by some robot. By the idle time of such an algorithm we mean the maximum value of $I(p)$ taken over all points of the environment. Throughout the chapter we will assume that $\sum_{i=1}^{k} 2 r_{i}<1$, since otherwise agents could constantly 
observe the environment without need to move (we assume that the length of the curve is 1$)$.

\subsubsection{Outline and Results}

In Section 4.2, we show that a version of the cyclic strategy is optimal for patrolling a closed curve. In Section 4.3, we prove that the partition strategy achieves the optimal idle time for open fence patrolling. To this end we show that any patrolling algorithm using robots equipped with visibility may be converted to a strategy for robots without visibility achieving at least the same idle time on an open fence shortened by twice the sum of all robots' visibility radii. This could suggest that the patrolling problem for robots with visibility is equivalent to patrolling with zero visibility robots considered elsewhere. However, the hardness of the problem for general graphs shown in Section 4.4, in view of the polynomial solution from [35] contradicts this supposition. In Sections 4.2.2 and 4.3.2, we discuss the cases of two robots with distinct speeds and visibility radii. In Section 4.3.2, we show that it is possible to extend the proof of optimality of the partition strategy (cf. [30, 58]) to the case of two visibility-equipped, distinct-speed robots. However, the example from Section 4.2.2 shows that for circle patrolling with two distinct-speed robots the cyclic strategy from [30] is no longer optimal when the robots are equipped with visibility. This is another evidence that patrolling with visibility-equipped robots presents new challenges, even for the case of robots with the same maximal speed. 


\subsection{Closed Fence Patrolling}

In this section we investigate patrolling of a circle (closed curve). First we give the optimal patrolling for the case of any number of robots with identical speeds. In the second subsection we briefly discuss the case of the robots with distinct speeds.

\subsubsection{Robots with Equal Speeds}

We start by considering the case of agents with equal speeds. We will assume that the maximum speed of each agent is equal to 1 . Recall that 1 is also the length of the environment i.e., we consider unit circles and unit intervals.

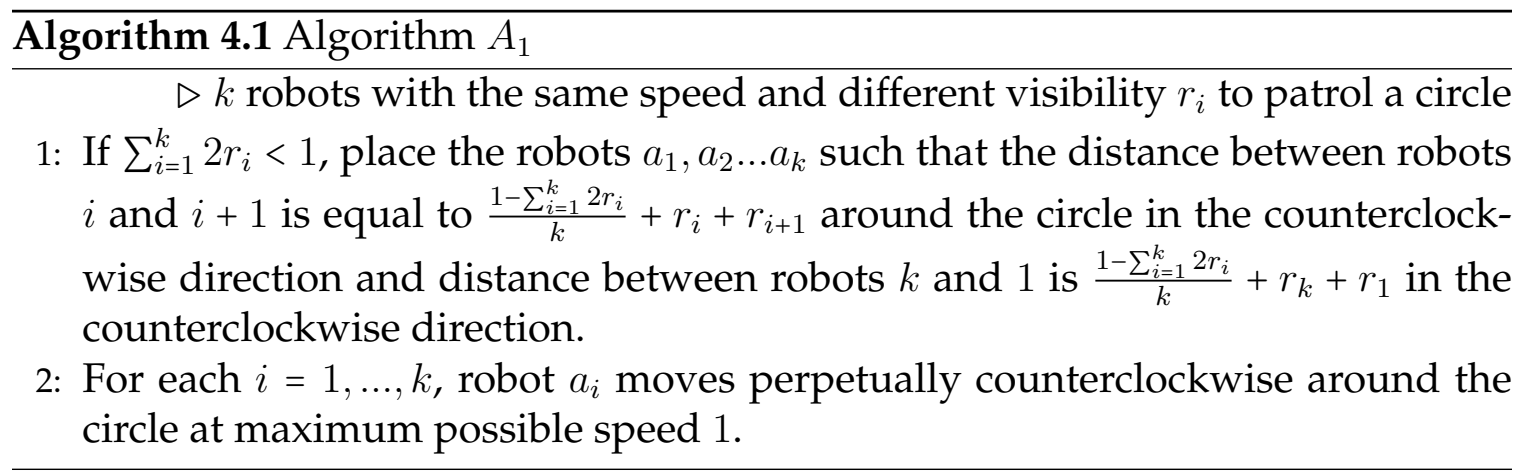

Proposition 4.2.1 Algorithm $A_{1}$ achieves the idle time $I=\frac{1-\sum_{i=1}^{k} 2 r_{i}}{k}$.

Proof. We need to show that for any point $p$, the longest contiguous interval of time when $p$ is not observed by any robot has length at most $\frac{1-\sum_{i=1}^{k} 2 r_{i}}{k}$. Observe that all robots are moving in the same direction with the same speed 1 . Thus the distances between robots and order of robots does not change. Moreover we can observe from the definition of the algorithm that distances between extremal points visible by neighboring robots is equal to $\frac{1-\sum_{i=1}^{k} 2 r_{i}}{k}$ and is also constant in time. 
Assume that point $p$ is not observed in the interval $I^{*}=\left[t_{1}, t_{2}\right]$. Time $t_{1}$ is the last moment of time when $p$ is observed by a robot $i$ and point $t_{2}$ is a first moment when $p$ is observed by a robot $i+1$. Thus during time interval $I^{*}$ point $p$ is between the regions of visibilities of robots $i$ and $i+1$. By the definition of the algorithm the distance between extremal points visible by robot $i$ and $i+1$ is constant and equal to $\frac{1-\sum_{i=1}^{k} 2 r_{i}}{k}$. Thus the length of the interval $I^{*}$ is exactly equal to $\frac{1-\sum_{i=1}^{k} 2 r_{i}}{k}$ which implies that the idle time of algorithm $A_{1}$ equals to $\frac{1-\sum_{i=1}^{k} 2 r_{i}}{k}$.

Theorem 4.2.2 Any patrolling algorithm for $k$ robots with speeds $v_{1}, \ldots, v_{k}$ and visibilities $r_{1}, \ldots, r_{k}$, patrolling unit circle achieves idle time at least $I_{\text {opt }} \geq \frac{1-\sum_{i=1}^{k} 2 r_{i}}{\sum_{i=1}^{k} v_{i}}$.

Proof. Consider any algorithm $A$ and its idle time $I_{A}$. Take any moment of time $t$. Regardless of the positions of the robots, the total length of the subset of the circle being within the radius of visibility of some robot is at most $\sum_{i=1}^{k} 2 r_{i}$. Thus the total length of points not being observed at time $t$ is $1-\sum_{i=1}^{k} 2 r_{i}$. Denote this set of not observed points at time $t$ by $U$. Take the interval of time

$$
I^{*}=\left[t, t+\frac{1-\sum_{i=1}^{k} 2 r_{i}}{\sum_{i=1}^{k} v_{i}}-\epsilon\right],
$$

for any $\epsilon>0$. The interval has length $\frac{1-\sum_{i=1}^{k} 2 r_{i}}{\sum_{i=1}^{k} v_{i}}-\epsilon$ thus the set of all points from set $U$ patrolled by the robot $i$ within time interval $I^{*}$ has length at most $v_{i} \frac{1-\sum_{i=1}^{k} 2 r_{i}}{\sum_{i=1}^{k} v_{i}}-v_{i} \epsilon$. Thus the set of all points from set $U$ patrolled by all robots within interval $I^{*}$ has total length at most

$$
\sum_{i=1}^{k} v_{i} \frac{1-\sum_{i=1}^{k} 2 r_{i}}{\sum_{i=1}^{k} v_{i}}-\sum_{i=1}^{k} v_{i} \epsilon=1-\sum_{i=1}^{k} 2 r_{i}-\sum_{i=1}^{k} v_{i} \epsilon=|U|-\sum_{i=1}^{k} v_{i} \epsilon<|U|
$$


Since within time interval $I^{*}$ the robots are unable to patrol all points from set $U$ thus the idle time of algorithm $A$ is bounded from below by the length of the interval $I^{*}$ :

$$
I_{A} \geq\left|I^{*}\right|=\frac{1-\sum_{i=1}^{k} 2 r_{i}}{\sum_{i=1}^{k} v_{i}}-\epsilon
$$

Therefore the claim of the theorem is obtained by passing to the limit $\epsilon \rightarrow 0$.

Corollary 4.2.3 Algorithm $A_{1}$ achieves an optimal idle time in the case of robots with equal speeds and possibly different visibilities.

\subsubsection{Robots with Different Speeds}

In the case of equal speeds our results are the same as for the problem without visibility on a circle of length $1-\sum_{i=1}^{k} 2 r_{i}$. It turns out that this is not the case any more for the case of different speeds. Consider the optimal algorithm for two robots without visibilities with speeds $v_{1}>v_{2}$. It is either an algorithm where both robots are at antipodal positions and move with the slower speed $v_{2}$ or it is an algorithm where the faster robot goes around the circle with his maximum speed $v_{1}$ and the movement of the slower robot irrelevant. It was proven in [30] that the idle time of such algorithm is optimal. But in our problem in the case of different visibilities in some cases neither of these algorithms is optimal. In particular, when one robot is very fast with small visibility radius, while the other robot is slow but it has a large visibility radius. In this case, the partition strategy (when both robots zigzag, protecting two interior-disjoint segments of the circle) may give a better 
idle time bound than those obtained by the two algorithms mentioned above. It is easy to verify that this is the case for $v_{1}=5, r_{1}=1 / 12, v_{2}=1$ and $r_{2}=1 / 3$.

\subsection{Open Fence Patrolling}

In this section we investigate patrolling of a segment. First we give the optimal patrolling for the case of any number of robots with identical speeds. In the second subsection we give the optimal algorithm for the case of two robots with distinct speeds.

\subsubsection{Robots with Equal Speeds}

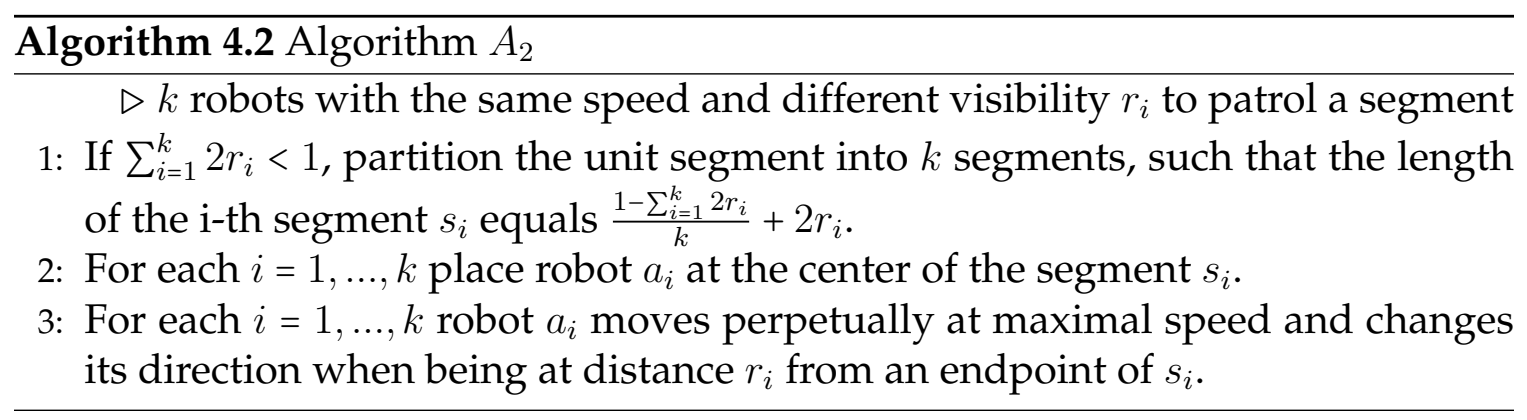

Proposition 4.3.1 If $\sum_{i=1}^{k} 2 r_{i}<1$ then patrolling algorithm $A_{2}$ achieves idle time $I=2 \frac{1-\sum_{i=1}^{k} 2 r_{i}}{k}$.

Proof. Since each robot covers a non-overlapping sub-segment of the unit segment (except for the common endpoint, which may be visited by two robots) the interior points of each sub-segment $s_{i}$ are visited by the same robot $a_{i}$. The infimum of the frequency of visits of point $x$ inside $s_{i}$ is achieved for $x$ being its endpoint. Since 
between two consecutive visits to the endpoint $x$ of $s_{i}$ robot $a_{i}$ traverses, using its speed $v_{i}$, the sub-segment $s_{i}$ of length $\frac{1-\sum_{i=1}^{k} 2 r_{i}}{k}$ twice, the idle time of such a point is $I=2 \frac{1-\sum_{i=1}^{k} 2 r_{i}}{k}$.

We want to prove optimality of the algorithm $A_{2}$. For any patrolling algorithm $A^{v i s}$ with visibility, operating on a segment of length 1 we will construct an algorithm $A^{\text {zis }}$ with no visibility, working on a segment of length $1-\sum_{i=1}^{k} 2 r_{i}$. The construction will ensure that the idle time of $A^{\text {vis }}$ is bigger than or equal to the idle time of $A^{z i s}$. Then, since it is straightforward to show the optimal algorithm for robots with the same speed and no visibility, we will obtain a desired lower bound.

Take any algorithm $A^{v i s}$ for $k$ robots with different visibilities $r_{1}, r_{2}, \ldots, r_{k}$ and the same speed 1 working on the line segment of length 1 . Assume that $\sum_{i=1}^{k} 2 r_{i}<1$. Denote by $A_{i}^{v i s}(t)$ the position of robot $i$ in time $t$. Define functions $L_{i}(t)=A_{i}^{v i s}(t)-r_{i}$ and $R_{i}(t)=A_{i}^{v i s}(t)+r_{i}$ for any robot $i$ and time $t$. Function $L_{i}(t)$ denotes the leftmost point visible to robot $i$ in time $t$, except the case when robot $i$ is too close to the left endpoint 0 . Similarly, function $R_{i}(t)$ denotes the rightmost point visible to robot $i$ in time $t$, except the case when robot $i$ is too close to the right endpoint 1 .

Definition of mobile intervals. A mobile interval is a pair of functions $\langle L(t), R(t)\rangle$ denoting its left and right endpoints respectively. The first step of our construction is the definition of a set of mobile intervals on the line. The goal of the construction is to obtain a dynamic process such that at any moment of time the union of all mobile intervals covers all points that are visible to all robots. We will require that mobile intervals move with speed not exceeding 1 and the total length of the intervals is always equal to $\sum_{i=1}^{k} 2 r_{i}$. If at some time $t$ for all $i \in\{1,2, \ldots, k\}$, the 
length of the set of points visible to a robot $i$ is equal to $2 r_{i}$, (no robot is too close to the endpoint) and all sets of visible points are disjoint (the visibility regions of two robots are always interior-disjoint), then mobile intervals will simply have $k$ intervals with lengths equal to areas of visibilities. However, for such time moments $t$ when the areas of visibilities of the robots are overlapping then the mobile intervals at time $t$ will cover some points that are not being observed by any robot. Now we will present an algorithm (Algorithm 4.3) that determines the positions of mobile intervals depending on the positions of robots in the algorithm $A^{\text {vis }}$ at any time moment $t$. If $t$ is the real variable denoting time then $J(t)$ is a dynamic process in which the mobile intervals are moving on the interval $[0,1]$. In the following lemma we prove some properties of this process.

Lemma 4.3.2 At any time moment $t$, the sequence of intervals $J(t)$ returned by the Algorithm 4.3 satisfies

(1) the intervals from $J(t)$ cover all points visible to the robots in the algorithm $A^{\text {vis }}$ at time $t$,

(2) the intervals from $J(t)$ are interior-disjoint and have total length equal to $\sum_{i=1}^{k} 2 r_{i}$,

(3) the velocity of any interval $j \in J(t)$ is either equal to velocity of some robot a or it is equal to 0.

Proof. Observe that the output $J(t)$ of Algorithm 4.3 is a sequence of intervals that are disjoint because the while loop in lines $8-14$ is executed until there are no more overlapping intervals. Moreover initially (line 1) the intervals cover all points that are visible to all robots and any operation in the Algorithm 4.3 preserves this 


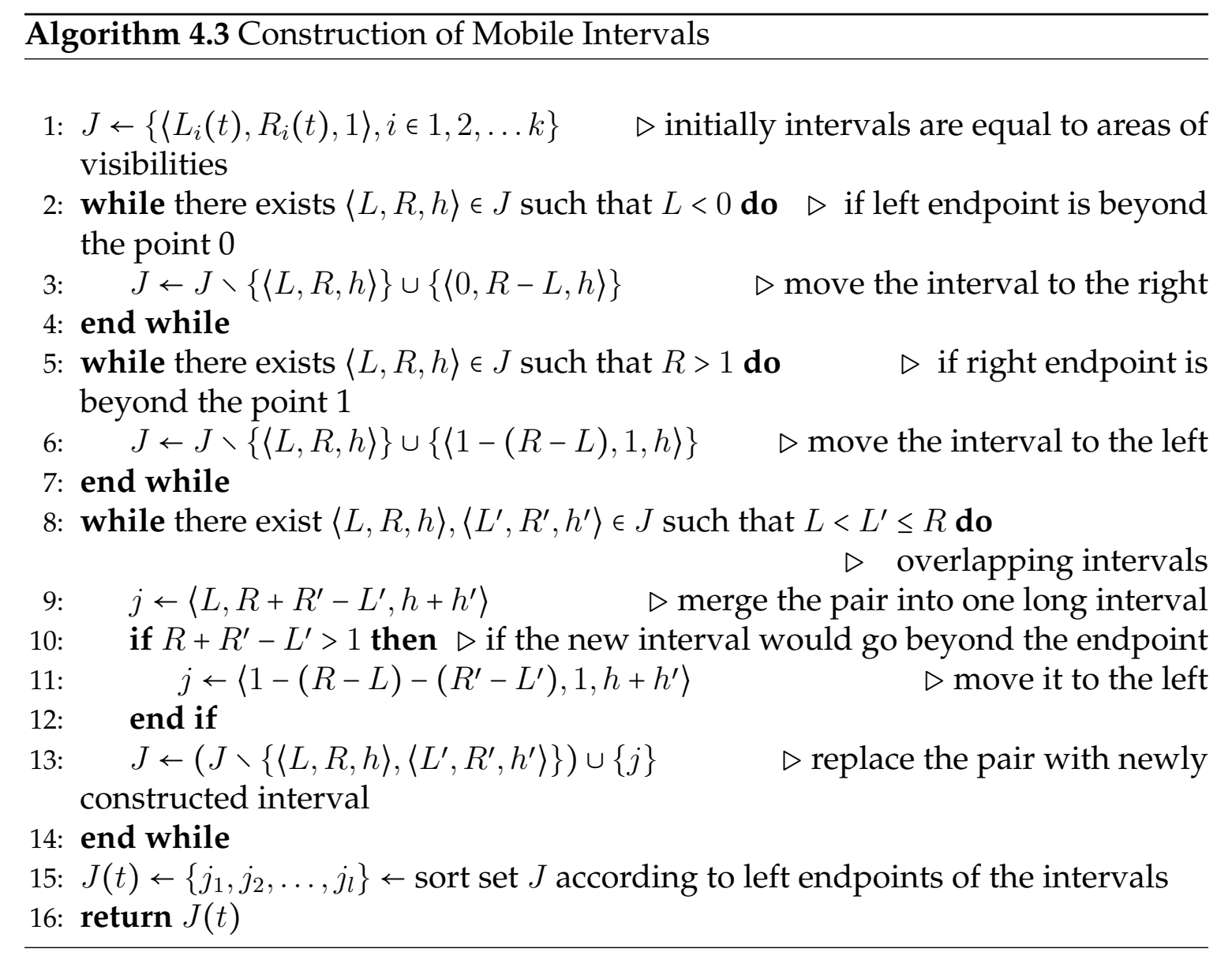

property. Thus the output $J(t)$ is a sequence of intervals that cover all points that are visible to robots in algorithm $A^{\text {vis }}$. And since the intervals are interior-disjoint they have total length $\sum_{i=1}^{k} 2 r_{i}$. To prove (3) let $L$ be the left endpoint of interval $j$. We can find $\delta_{t}>0$ such that for any $t^{\prime} \in\left[t, t+\delta_{t}\right]$, the Algorithm 4.3 performs exactly the same operations on intervals. Consider two cases. Either $L$ is equal to the left endpoint of the area of visibility of some robot $a$ (i.e. $L=L_{a}(t)$ ). In this case the left endpoint of the interval $j$ for any $t^{\prime} \in\left[t, t+\delta_{t}\right]$ is equal to the left endpoint of the area of visibility of robot $a$. Thus the velocity of interval $j$ in time $t$ is equal to the velocity of robot $a$. In the other case the position of $j$ for any $t^{\prime} \in\left[t, t+\delta_{t}\right]$ is 
constant thus the velocity of $j$ is equal to 0 .

Thus any mobile interval either moves with the same speed and direction as some robot, or merges with other interval or splits into multiple intervals. Based on the positions of the intervals we can define positions of the robots in algorithm $A^{\text {zis }}$ at any step $t$.

Definition of the algorithm $A^{\text {zis. }}$. Take any time moment $t$. Based on the positions of robots in the algorithm $A^{\text {vis }}$ at this moment we construct the mobile intervals $J(t)$. Consider the output of the Algorithm 4.3, namely the set $J(t)=\left\{j_{1}, j_{2}, \ldots, j_{l}\right\}$. Each $j_{i}$ is a tuple $j_{i}=\left\langle L_{i}, R_{i}, h_{i}\right\rangle$, where $L_{i}$ is the left endpoint, $R_{i}$ is the right endpoint and $h_{i}$ is the number of robots whose areas of visibilities are being covered by interval $j_{i}$. Recall that $J(t)$ is sorted thus $L_{1}<R_{1}<L_{2}<R_{2}<\cdots<L_{l}<R_{l}$. To

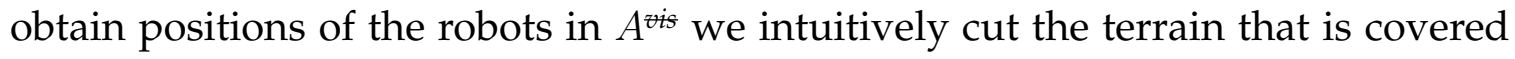
by the mobile intervals (see Figure 4.1).

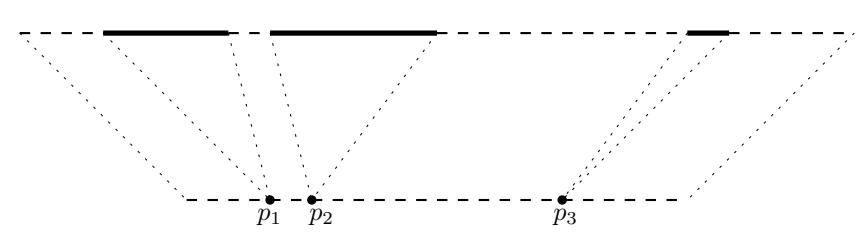

FIGURE 4.1: Positions of robots in the algorithm $A^{\text {. }}$.

The amount of terrain that is left is $1-\sum_{i=1}^{k} 2 r_{i}$. Define points $p_{1}=L_{1}, p_{i}=L_{i}-\sum_{j=1}^{i-1}\left(R_{j}-\right.$ $L_{j}$ ) for $i=2,3, \ldots, l$ as the total length of all points to the left of point $L_{i}$ not covered by any mobile interval. Points $p_{i}$ will be positions of the robots in the algorithm $A^{*}$. Number of robots located at the point $p_{i}$ will be equal to $h_{i}$. Let $s_{i}=\sum_{j=1}^{i} h_{j}$ for 
$i=1,2, \ldots, l$. Positions of robots in algorithm $A^{\text {vis }}$ are defined as follows.

$$
\begin{aligned}
A_{a}^{\text {zis }}(t) & =p_{1} \quad \text { for } a=1,2, \ldots, s_{1} \\
A_{a}^{z i s}(t) & =p_{2} \quad \text { for } a=s_{1}+1, s_{1}+2, \ldots, s_{2} \\
\vdots & \\
A_{a}^{z i s}(t) & =p_{l} \quad \text { for } a=s_{l-1}+1, s_{l-1}+2, \ldots, s_{l}
\end{aligned}
$$

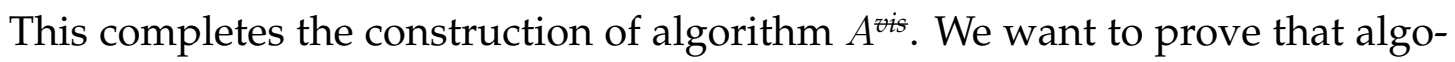
rithm $A^{\text {has }}$ idle time not bigger than $A^{v i s}$. First we need to show that robots in the algorithm $A^{\text {move with speeds not exceeding } 1 .}$

Lemma 4.3.3 For any time moment $t$ and robot $a_{i}$

(1) $A_{i}^{\text {चis }}(t) \in\left[0,1-\sum_{i=1}^{k} 2 r_{i}\right]$ ，

(2) speed of robot $a_{i}$ is at most 1 ,

(3) the trajectory followed by robot $a_{i}$ is continuous.

Proof. First we want to prove part (1). Recall that the positions of robots in algorithm $A^{\text {zis }}$ at time $t$ are defined as points $p_{i}$ satisfying $p_{i}=L_{i}-\sum_{j=1}^{i-1}\left(R_{j}-L_{j}\right)$, for $i=1,2, \ldots l$, where $R_{i}$ and $L_{i}$ are positions of the endpoints of the mobile intervals at time $t$. Intervals are ordered (line 15 of the Algorithm 4.3) and nonoverlapping (Lemma 4.3.2(2)) thus $L_{1}<R_{1}<L_{2}<R_{2}<\cdots<L_{l}<R_{l}$. Observe that $p_{i+1}-p_{i}=L_{i+1}-R_{i}>0$, thus the sequence $p_{1}, p_{2}, \ldots, p_{l}$ is increasing. Since $p_{1} \geq 0$ we 
have $A_{i}^{2 i s}(t) \geq 0$. Then

$$
\begin{aligned}
p_{l} & =L_{l}-\sum_{j=1}^{l-1}\left(R_{j}-L_{j}\right) \\
& =R_{l}-\sum_{j=1}^{l}\left(R_{j}-L_{j}\right) \\
& \leq 1-\sum_{i=1}^{k} 2 r_{i},
\end{aligned}
$$

where in the last inequality we use the fact that the total length of the mobile intervals is equal to $\sum_{i=1}^{k} 2 r_{i}$ (which is true by Lemma 4.3.2(2)). Since $p_{l}$ is the largest value $p_{i}$ we have that $A_{i}^{z i s}(t) \leq 1-\sum_{i=1}^{k} 2 r_{i}$. The proof of part (2) is a consequence of Lemma 4.3.2(3).

It remains to prove part (3). Observe that movement of mobile intervals is continuous thus movement of the positions $p_{i}$ is also continuous. It is left to observe that a robot may not "jump" between positions $p_{i}$. Indeed, it is true because the number of robots assigned to mobile intervals (variables $h_{j}$ in the Algorithm 4.3) changes only if mobile intervals join or split.

Lemma 4.3.3 shows that algorithm $A^{* i s}$ is a correct patrolling algorithm for segment of length $1-\sum_{i=1}^{k} 2 r_{i}$ and robots with the same speed 1 . Now we need to show that the transformation does not increase the idle time.

Lemma 4.3.4 The idle time of algorithm $A^{z i s}$ is not larger than the idle time of algorithm $A^{\text {vis. }}$

Proof. Let $I^{v i s}$ and $I^{z i s}$ be idle times of algorithms $A^{v i s}$ and $A^{z i s}$ respectively. Consider the parallel execution of both algorithms $A^{v i s}$ and $A^{\text {vis. }}$. Take a vertex $v \epsilon$ $\left[0,1-\sum_{i=1}^{k} 2 r_{i}\right]$ and an interval of time $\left[t_{1}, t_{2}\right]$ and assume that $v$ is not visited in 


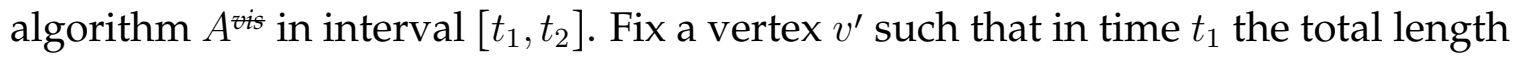
of the regions not covered by the mobile intervals to the left of $v^{\prime}$ is equal to $v$, and $v^{\prime}$ is a leftmost point with such property. Since $v^{\prime}$ is leftmost then it is either a left endpoint of some mobile interval or it is not covered by any mobile interval at time $t_{1}$. If $v^{\prime}$ was a left endpoint of some mobile interval then by the definition of algorithm $A^{z i s}$, the corresponding point $v$ would be visited at time $t_{1}$ by algorithm $A^{* i s}$. Thus $v^{\prime}$ is not covered by any mobile interval at time $t_{1}$. Using the same argument we can prove that $v^{\prime}$ is not covered by any mobile interval at any moment from time interval $\left[t_{1}, t_{2}\right]$. Since mobile intervals at any time cover all points visible to robots in $A^{v i s}$ then $v^{\prime}$ is also not refreshed by any robot in algorithm $A^{v i s}$. Now if $I^{*}>I^{v i s}$ then there exists a vertex $v^{*}$ and a closed interval $\left[t^{*}, t^{* *}\right]$ of length larger

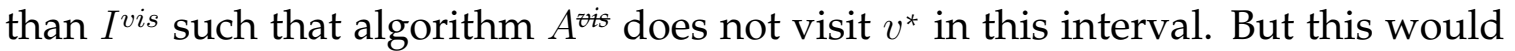
imply existence of a point not refreshed by $A^{v i s}$ in the interval $\left[t^{*}, t^{* *}\right]$ which is a contradiction since length of $\left[t^{*}, t^{* *}\right]$ is larger than $I^{v i s}$.

Theorem 4.3.5 The optimal traversal algorithm for $k$ robots with the same speed and different visibility, patrolling unit segment $S=[0,1]$ achieves idle time $I_{\text {opt }}=2 \frac{1-\sum_{i=1}^{k} 2 r_{i}}{k}$.

Proof. By our construction and Lemma 4.3.4 the existence of an algorithm $B^{\text {vis }}$ for robots with visibility operating on a segment $[0,1]$ achieving idle time $I$ implies existence of an algorithm $B^{\text {fis }}$ for robots with no visibility operating on a segment $\left[0,1-\sum_{i=1}^{k} 2 r_{i}\right]$ with idle time at most $I$. The optimal algorithm for patrolling without visibility of a segment of length $1-\sum_{i=1}^{k} 2 r_{i}$ achieves idle time $2 \frac{1-\sum_{i=k}^{k} 2 r_{i}}{k}$ [58]. Thus there cannot exist an algorithm with visibility achieving idle time better than $2 \frac{1-\sum_{i=1}^{k} 2 r_{i}}{k}$ on the unit segment. Hence $2 \frac{1-\sum_{i=1}^{k} 2 r_{i}}{k}$ is a lower bound on the idle time 
for algorithms with visibility on the unit segment. By Proposition 4.3.1, algorithm $A_{2}$ achieves the optimal idle time.

\subsubsection{Robots with Different Speeds}

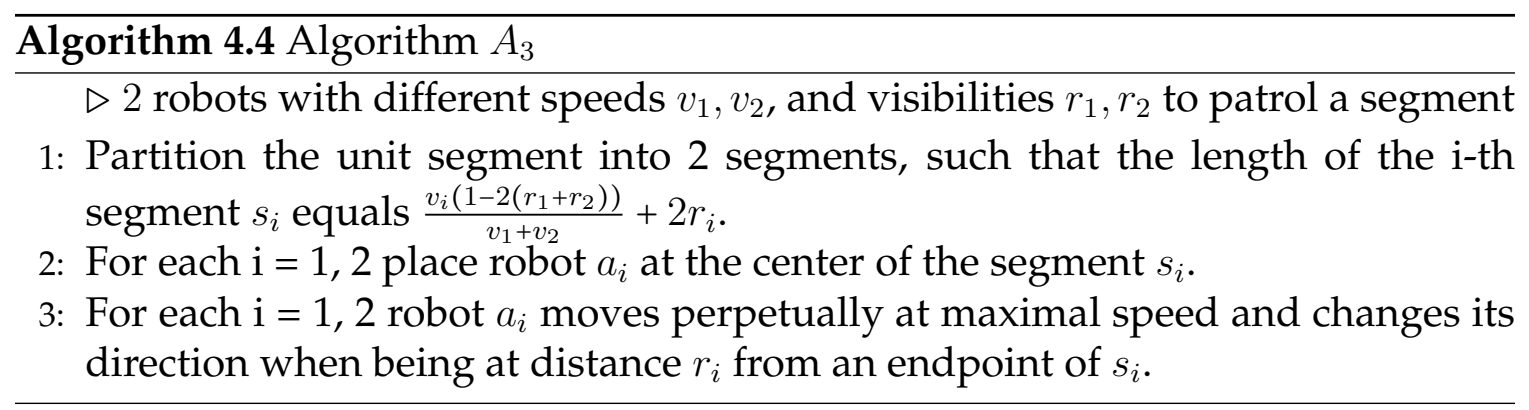

Proposition 4.3.6 Traversal algorithm $A_{3}$ achieves idle time $I=2 \frac{1-2\left(r_{1}+r_{2}\right)}{v_{1}+v_{2}}$.

Proof. Since each robot covers a non-overlapping sub-segment of the unit segment (except for the common endpoint, which may be visited by two robots) the interior points of each sub-segment $s_{i}$ are visited by the same robot $a_{i}$. The infimum of the frequency of visits of point $x$ inside $s_{i}$ is achieved for $x$ being its right endpoint. Take a point $x-\epsilon$, for $\epsilon>0$. Since between two consecutive visits to the endpoint $x$ of $s_{i}$ robot $a_{i}$ traverses, using its speed $v_{i}$, the sub-segment $s_{i}$ of length $\frac{v_{i}\left(1-2\left(r_{1}+r_{2}\right)\right)}{v_{1}+v_{2}}-\epsilon$ twice, the idle time of such a point is $I=2 \frac{1-2\left(r_{1}+r_{2}\right)}{v_{1}+v_{2}}-\epsilon$. As $\epsilon$ may be as close to 0 as we wish, we obtain the claim of our lemma.

We prove below that the algorithm $A_{3}$ is optimal.

Theorem 4.3.7 The optimal traversal algorithm for two robots with different speed and visibility, patrolling unit segment $S=[0,1]$ achieves idle time $I_{\text {opt }}=2 \frac{1-2\left(r_{1}+r_{2}\right)}{v_{1}+v_{2}}$. 
Proof. We suppose, by contradiction, that there exists an algorithm A with an idle time of $I_{A}=I-\epsilon$ for some $\epsilon>0$. Without loss of generality, we may assume that $v_{1} \leq v_{2}$. Observe that $a_{1}$ must see one of the endpoints $(0,1)$. By symmetry suppose that $a_{1}$ sees endpoint 0 at some time $t_{1}$. Let $L_{1}=r_{1}, R_{1}=r_{1}+\frac{1-2\left(r_{1}+r_{2}\right)}{v_{1}+v_{2}} v_{1}$, $L_{2}=1-r_{2}-\frac{1-2\left(r_{1}+r_{2}\right)}{v_{1}+v_{2}} v_{2}, R_{2}=1-r_{2}$, and $B=R_{1}+r_{1}=L_{2}-r_{2}$.

At time $t_{1}, a_{1}$ is within $\left[0, L_{1}\right]$. Considering the speed of $a_{1}$,

$$
v_{1} \frac{T-\epsilon}{2}+r_{1}<|B-0|
$$

and $a_{1}$ cannot see $B$ within time $\left[t_{1}-\frac{T-\epsilon}{2}, t_{1}+\frac{T-\epsilon}{2}\right]$. So, $a_{2}$ has to see $B$ at some time $t_{2}$. We will show that neither $a_{2}$ nor $a_{1}$ can see 1 within $\left[t_{2}-\frac{T-\epsilon}{2}, t_{2}+\frac{T-\epsilon}{2}\right]$. Considering the speed of $a_{2}, v_{2} \frac{T-\epsilon}{2}+r_{2}<|B-1|$ and $a_{2}$ cannot see 1 within the time interval $\left[t_{2}-\frac{T-\epsilon}{2}, t_{2}+\frac{T-\epsilon}{2}\right]$. Now, we show that $a_{1}$ cannot see 1 neither. Since $\left|t_{1}-t_{2}\right|<\frac{T}{2}$, the rightmost point $p$ at which $a_{1}$ can be at time $t_{2}$ is $p<L_{1}+\frac{T}{2} v_{1}$. We show that from the moment when $a_{1}$ sees point $p$, it is not possible for $a_{1}$ to see 1 within time $\frac{T-\epsilon}{2}$, this would prove that in interval $\left[t_{2}-\frac{T-\epsilon}{2}, t_{2}+\frac{T-\epsilon}{2}\right], a_{1}$ cannot see 1 . The rightmost point $q$ that $a_{1}$ can visit within time $t_{2}+\frac{T-\epsilon}{2}$ is $q<p+\frac{T-\epsilon}{2} v_{1}$. Hence, the rightmost point that $a_{1}$ can see within time $t_{2}+\frac{T-\epsilon}{2}$ is

$$
q+r_{1}<L_{1}+\frac{T}{2} v_{1}+\frac{T-\epsilon}{2} v_{1}=L_{1}+T v_{1}-\frac{\epsilon}{2} v_{1}
$$

which proves the theorem. 


\subsection{Arbitrary Graphs Patrolling}

Let us recall the definition of the PARTITION problem.

Instance: Finite set $A$ and size $s(a) \in \mathbb{Z}^{+}$for each $a \in A$.

Question: Is there a subset $A^{\prime} \subset A$ such that $\sum_{a \in A^{\prime}} s(a)=\sum_{a \in A \backslash A^{\prime}} s(a)$ ?

The PARTITION problem remains NP-complete even if we require that $\left|A^{\prime}\right|=|A| / 2$ [49]. We will refer to the PARTITION problem with this additional condition as RESTRICTED PARTITION problem.

Theorem 4.4.1 For arbitrary graphs the problem of deciding, for any set of robots $a_{1}, a_{2}, \ldots, a_{k}$ with equal speeds and different visibility ranges, whether there exists a patrolling algorithm with idle time 0 is NP-hard.

Proof. We want to reduce the partition problem, which is known to be NP-hard. Assume that there exists an algorithm $A$ answering, given a graph and a set of robots, whether it is possible to deploy the robots in the graph such that their visibility ranges cover the entire graph (i.e. the idle time is 0 ). Let a multiset of integers $S=\left\{x_{1}, x_{2}, \ldots, x_{n}\right\}$ be an instance from the PARTITION problem. Let $s=\sum_{i=1}^{n} x_{i}$. We will construct an instance of the patrolling problem as follows. Take $n+1$ robots $a_{1}, a_{2}, \ldots a_{n+1}$ and let the radius of visibility the of $i$-th robot be equal to $x_{i} / 2$ for $i=1,2, \ldots, n$. Let the radius of visibility of $n+1$-st robot be equal to $m=\max \left\{x_{1}, x_{2}, \ldots, x_{n}\right\}+1$. Let graph $G$ be a "cross" with two arms of length $m$ and two of length $s / 2+m$ (see figure 4.2). A patrolling algorithm with idle time 0 is simply a placement of robots such that every point is observed (i.e. within radius of visibility) for some robot. The horizontal line of the cross $G$ has length 


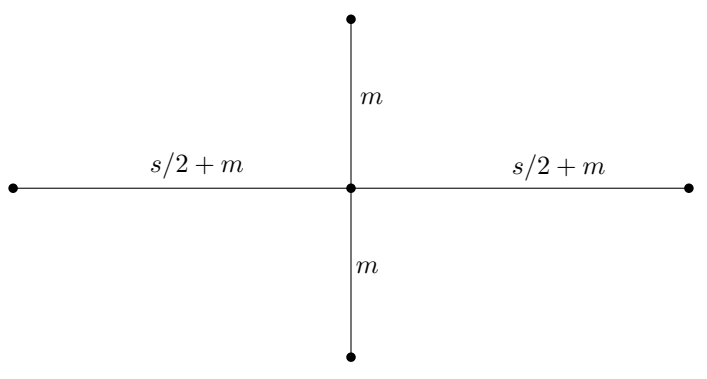

FIGURE 4.2: Graph $G$.

$s+2 m$ which is equal to sum of diameters of visibilities of all robots. Thus any algorithm which places any robot not on the horizontal line will not have idle time 0 . Moreover to cover the vertical line an algorithm has to place the $n+1$-st robot on the crossing. To cover the remaining part of $G$ an algorithm has to divide the robots into two groups with equal total length of visibility $s / 2$. Thus $G$ can be patrolled with idle time 0 if and only if there is a solution to the partition problem. Thus having algorithm $A$ we can construct an algorithm solving the partition problem.

When the idle time is strictly positive the construction of the example showing NP-hardness is more involved.

Theorem 4.4.2 For any fixed I and for some graphs the problem of deciding, for any set of robots $a_{1}, a_{2}, \ldots, a_{k}$ with equal speeds and different visibilities, whether there exists a patrolling algorithm with idle time at most I is NP-hard.

Proof. Fix any $I$. Assume that there exists a polynomial algorithm deciding for any set of robots whether it is possible to patrol a graph obtaining idle time at most $I$. We want to show that it would imply existence of a polynomial algorithm for the RESTRICTED PARTITION problem. 
Let a multi-set of integers $S=\left\{x_{1}, x_{2}, \ldots, x_{2 k}\right\}$ be an instance from the RESTRICTED PARTITION problem. The sum of all values $x_{i}$ is such that $\sum_{j=1}^{2 k} x_{j}=I / 2$. We construct an instance of the patrolling problem consisting of $2 k+1$ robots in the following way. Let the radius of visibility $r_{i}$ of the $i$-th robot be $r_{i}=x_{i}$ for $i=1,2, \ldots, 2 k$ and let the radius of visibility of $(2 k+1)$-st robot (call it $\left.a^{*}\right)$ be $r_{2 k+1}=I$. We take the following graph $H$ (see Figure 4.3). The length of inter-

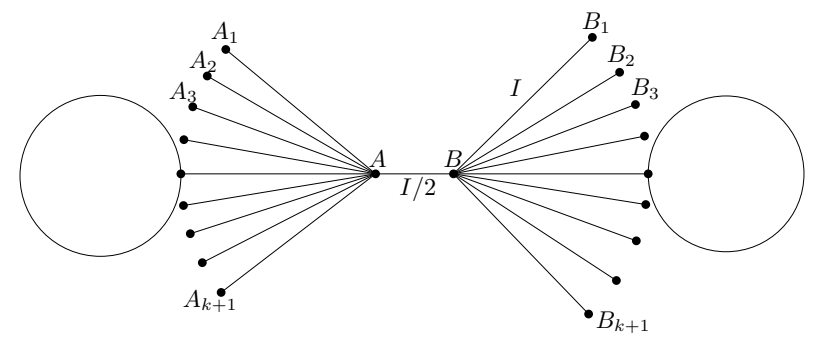

FIGURE 4.3: Graph $H$.

val $A B$ is $I / 2$, the length of each interval $A A_{i}$ is $I$ for $i=1,2, \ldots k+1$, the length of each interval $B B_{i}$ is $I$ for $i=1,2, \ldots k+1$ and the perimeter of each circle is $k I+I / 2$. We ask if such a collection of robots can patrol graph $H$ with idle time at most $I$.

We will argue that the answer can be yes if and only if the RESTRICTED PARTITION problem has a solution. First observe that the robot $a^{*}$ has to walk perpetually between nodes $A$ and $B$. Note that a robot has to visit node $A$ at least once in every interval of time of length $I$. If it does not visit $A$ in some interval of length $I$ then in this interval some of the nodes $A_{1}, A_{2}, \ldots A_{k+1}$ will not be patrolled by any robot. Since the distance between two nodes $A_{i}, A_{j}(i \neq j)$ is $2 I$ thus any robot different from $a^{*}$ can patrol at most one node among $A_{1}, A_{2}, \ldots A_{k+1}$ in time $I$. And since we have $k+1$ nodes $A_{i}$ then $a^{*}$ has to visit node $A$ once every $I$ time steps. Since the same applies to $B$, robot $a^{*}$ has to perpetually walk between $A$ and $B$. 
To patrol the circles we have to use the remaining robots. First note that, if during some time interval of size $I, k-1$ or less robots will be on one of the circles then the idle time must be larger than $I$. This is because $k-1$ robots can patrol in time $I$ intervals of total length at most $(k-1) I+I / 2$ (because $I / 2$ is an upper bound on the total length of the visibilities) which is less than the length of the circle. Therefore, idle time $I$ will be achieved if and only if the two sums of diameters of visibilities of groups assigned to both circles are the same. But this corresponds exactly to the solution of the RESTRICTED PARTITION problem.

\subsection{Conclusion and Open Problems}

In this chapter, we investigated the problem of mobile robots with visibility patrolling a curve. We gave optimal patrolling algorithms for the case of circle patrolling and segment patrolling when all robots have the same maximum speed. We also discussed briefly the case of distinct speeds, thus showing that the fence patrolling problem for robots with distinct visibility ranges is entirely different than the case of point-visibility robots. However, we proposed an optimal algorithm for open fence patrolling by two robots with distinct speeds and visibility ranges. If the underlying domain in which the robots operate is a general graph, then the patrolling problem for robots with distinct visibility (and the same speed) ranges is shown to be NP-hard; this contrasts sharply with point-visibility robots which has been known to have a polynomial-time solution [35].

Some interesting open problems are investigating the approximation algorithms for patrolling general graphs with robots equipped with visibility. We may also 
consider on-line (i.e., the environment to be patrolled is unknown ), and off-line (i.e., the environment to be patrolled is known) patrolling algorithms for robots with distinct visibilities and speeds. 


\section{Chapter 5}

\section{Patrolling Trees by Homogeneous Mobile Robots}

\subsection{Introduction}

In previous chapters, we considered variant models of heterogeneous mobile robots (faulty robots, and robots with different visibility) patrolling a given region. Although it may look straightforward, there is still no optimal patrolling algorithm for mobile robots with identical speed and visibility patrolling a general graph. Therefore, in this chapter we investigate patrolling a tree $T$, i.e., connected acyclic graph, by a team of homogeneous mobile robots with identical speed and zero visibility. The main problem of interest is the following: assume $k$ robots having the same maximum speed are patrolling a tree $T$. Give a patrolling algorithm that optimizes the idle time on $T$, denoted by $I(T, k)$. 


\subsubsection{Preliminaries and Notation}

Consider a geometric graph with $n$ vertices and $m$ edges and $k$ mobile robots initially placed on vertices of the graph. Let $\mathcal{A}$ be a patrolling algorithm, scheduling the movement of the robots at the same constant speed. For any algorithm $\mathcal{A}$ and any graph $G$ let $I_{\mathcal{A}}(G, k)$ denote the idle time of the algorithm $\mathcal{A}$ for $k$ robots on this graph; namely the supremum of "the time between successive visits of the robots at point $x$ following algorithm $\mathcal{A}^{\prime \prime}$, where the supremum is taken over all the points $x$ of all edges of the graph $G$. Finally, we define

$$
I(G, k):=\inf _{\mathcal{A}} I_{\mathcal{A}}(G, k)
$$

where the infimum is over all possible patrolling algorithms $\mathcal{A}$ for $G$.

\subsubsection{Outline and Results}

There is an off-line schedule placing the $k$ robots at specific initial positions on a geometric tree $T$ such that if the robots move perpetually at speed 1 it will achieve the optimal idle time $\frac{2|T|}{k}$ where $|T|$ is the sum of the length of the edges of $T$. Our main contribution in Section 5.2 is to show that such a schedule is an optimal one, i.e. that no other schedule can achieve a smaller idle time. We extend this to the graph tree where the patrolling robots are allowed to change direction only at vertices of the tree. In Section 5.3, we consider the on-line setting and construct examples of trees with port numberings on the nodes such that the resulting rotorrouter algorithm has competitive ratio at least 4/3. In addition, in Section 5.3.2, we 
perform experimental work indicating that in a random setting the rotor-router on tree graphs is nearly optimal.

\subsection{Off-Line Patrolling Algorithm}

This section is concerned with the idle time for patrolling trees with a given number of robots. We distinguish patrolling on two types of domains: geometric trees, and graph trees. In the former, edge lengths are real numbers and during their traversal the robots may change direction anywhere on vertices as well as in the interior of edges. In the latter, edge lengths are positive integers and during their traversal the robots may change direction only on vertices. Note that the graph tree model will prove useful in our study of experimental results in Section 5.3.2.

\subsubsection{Patrolling of Geometric Trees}

Let $|T|$ denote the sum of lengths of the edges of $T$. The main theorem is the following.

Theorem 5.2.1 (Idle Time for Trees) For any tree $T$ and any number $k$ of robots,

$$
I(T, k)=\frac{2|T|}{k} .
$$

This idle time is attained when $k$ robots traverse the tree at their maximum speed along an Eulerian cycle (i.e., the Chinese Postman Tour of tree), while at the same time ensuring that, during the traversal, consecutive robots remain equidistant on this cycle. 
Before proving the theorem we will prove several lemmas that will lead to the main result. As noted above, we assume throughout the discussion of the proof below that during their traversal the robots may change direction anywhere on vertices as well as in the interior of edges. Let $L$ denote the line graph. The first lemma is well known.

Lemma 5.2.2 (Idle Time for Lines) For $k$ robots on a line $L, I(L, k)=\frac{2|L|}{k}$, where $|L|$ is the length of the line $L$.

For a tree $T$ let $|T|$ denote the sum of the lengths of its edges. First we prove the upper bound in the following lemma.

Lemma 5.2.3 (Upper Bound for Trees) For any tree $T$ and any number $k$ of robots, $I(T, k) \leq \frac{2|T|}{k}$.

Proof. (Lemma 5.2.3) The upper bound $I(T, k) \leq \frac{2|T|}{k}$ is obvious since we can arrange the robots so that they traverse an Eulerian tour of the tree (staying equidistant) which has length $2|T|$.

To prove the lower bound, first we define the following useful concept.

Definition 1 The cumulative idle time on a tree $T$ is defined as $F_{T}(k):=k I(T, k)$, where $I(T, k)$ is the optimal idle time for $k$ robots on the tree $T$.

The main idea for the proof of the lower bound $I(T, k) \geq \frac{2|T|}{k}$ is based on proving two properties, namely:

1. monotonicity of the cumulative idle time with respect to doubling the number of robots (see Inequality (5.3) and Lemma 5.2.4), and 
2. validity of the lower bound on caterpillar trees for $k$ sufficiently large (see Lemma 5.2.6).

A combination of these two ideas will lead to the proof of the actual result. To this end, first we consider the monotonicity of the cumulative idle time.

Lemma 5.2.4 (Monotonicity of the Cumulative Idle Time) For any number of robots $k$,

$$
F_{T}(2 k) \leq F_{T}(k)
$$

Proof. (Lemma 5.2.4) Consider a patrolling algorithm $\mathcal{A}$ attaining the optimal idle time $I(T, k)$. Let $r$ be a given robot. For any real number $t \geq 0$, observe a trajectory traversed by robot $r$ according to algorithm $\mathcal{A}$ during the time interval $[t, t+I(T, k)]$ (of duration $I(T, k)$ ). Since during the patrolling the robot moves with speed at most 1 , the positions of the robot at times $t$ and $t+I(T, k)$ cannot be at distance bigger than $I(T, k)$, where the distance is measured along the edges of the tree.

Consider the $k$ original robots as they are moving according to an optimal algorithm $\mathcal{A}$ patrolling the tree with idle time $I(T, k)$. We now double the original number $k$ of robots by inserting $k$ additional robots patrolling the tree. The insertion procedure is as follows. Let the original robots be $r_{1}, r_{2}, \ldots, r_{k}$. For the current discussion lets use the notation $I:=I(T, k)$. Take a snapshot of the robots at some time, say $t$. Look at the robots at time $t+I / 2$ as the robots move to new positions. Take $k$ new robots $r_{1}^{\prime}, r_{2}^{\prime}, \ldots, r_{k}^{\prime}$ and place them in the positions previously occupied by robots $r_{1}, r_{2}, \ldots, r_{k}$, respectively, at time $t$. Call robot $r_{i}^{\prime}$ the follower of robot $r_{i}$, and similarly robot $r_{i}$ the master of robot $r_{i}^{\prime}$. The $k$ new robots $r_{1}^{\prime}, r_{2}^{\prime}, \ldots, r_{k}^{\prime}$ are given the following trajectory: 
- from that time on, have each robot $r_{i}^{\prime}$ copy faithfully the trajectory of its master, for $i=1,2, \ldots, k$.

It is clear that if an arbitrary point $p$ is visited by a robot $r$ at any time $t$ it will be visited again by its follower at time $t+I / 2$. Therefore the idle time $I(T, 2 k)$ for $2 k$ robots is at most half the idle time $I(T, k)$ for $k$ robots, i.e., $I(T, 2 k) \leq I(T, k) / 2$. After multiplying both sides by $2 k$, this implies that $2 k I(T, 2 k) \leq k I(T, k)$. This proves Inequality (5.3) and hence also Lemma 5.2.4.

Next we define a generalization of the concept of caterpillar that will be useful for the proof.

Definition $2 A d$-caterpillar tree is a tree in which all the vertices are within distance $d$ of a central path of the tree, where $d \geq 1$.

Thus, the well-known concept of caterpillar is identical to 1-caterpillar. The following lemma provides a useful property that will be useful for the proof of the main theorem.

Lemma 5.2.5 If $T$ is a $d$-caterpillar with $d \geq 2$ then there is a $(d-1)$-caterpillar $T^{\prime}$ which is a subtree of $T$ such that all the vertices of $T$ are within distance 1 of a node of $T^{\prime}$.

Proof. (Lemma 5.2.5) The proof is simple. The subtree $T^{\prime}$ is obtained from $T$ by removing all its leaves. The required property for $T^{\prime}$ follows easily.

Lemma 5.2.6 (A Lower Bound for Caterpillar Trees) For any caterpillar (i.e., 1-caterpillar) tree $T$, and for any real number $\epsilon>0$ there is a sufficiently large integer $k_{0}$ such that $I(T, k) \geq \frac{2|T|}{k}-\frac{2 \epsilon}{k}$, for all $k \geq k_{0}$. 
Proof. (Lemma 5.2.6) First observe that when two robots meet they can exchange their patrolling roles. Therefore without loss of generality we may assume that no two robots ever cross each other at any point on the tree when coming from opposite directions. Using this, it follows that for any leaf node of the tree a robot always has to stay within distance at most $I(T, k) / 2$ from this leaf node so as to maintain idle time $I(T, k)$. Thus a robot must be dedicated to patrolling each leaf; this allows us to chop a segment of length $I(T, k) / 2$ from each leaf whose adjacent edge length is at least $I(T, k) / 2$ without affecting the idle time of the tree. By chopping such segments of length $I(T, k) / 2$ recursively we are left with star graphs (having as centers internal nodes of the tree) and respective edges of lengths at most $I(T, k) / 2$. Further, since the upper bound $I(T, k) \leq \frac{2|T|}{k}$ holds, the quantity $I(T, k) / 2$ (which is an upper bound on the lengths of the edges of the stars) can be made as small as we wish by making $k$ sufficiently large.

Let $P$ be the central path of the caterpillar tree. The graph resulting from the procedure above consists of $P$ together with "stars of small weight". Consider the sum, say $\Delta$, of all the degrees of all the stars in the tree. Clearly, $\Delta$ depends only on the given tree $T$ and is otherwise independent of the number $k$ of robots. In addition, the sum of the lengths of the edges of all the stars is at most $\Delta \frac{I_{k}(T)}{2} \leq \Delta \frac{|T|}{k}$. Therefore for any $\epsilon>0$ we can select $k_{0}$ sufficiently large so that the sum of the lengths of all the edges of all the stars is at most $\Delta \frac{|T|}{k_{0}} \leq \epsilon$.

It is clear from the above discussion that $|P|+\epsilon \geq|T| \geq|P|$, for all $k \geq k_{0}$. From Lemma 5.2.2 we know that $I(P, k)=\frac{2|P|}{k}$, for any $k$. Thus, it is obvious that for all $\epsilon>0$ there is an integer $k_{0}$ such that for all $k \geq k_{0}$ we have that $I(T, k) \geq I(P, k)=$ $\frac{2|P|}{k} \geq \frac{2|T|}{k}-\frac{2 \epsilon}{k}$. This proves Lemma 5.2.6. 
Now we are ready to prove the lower bound for caterpillars. We prove the following.

Lemma 5.2.7 (Idle Time for Caterpillar Trees) For any caterpillar (i.e., 1-caterpillar) tree $T$ and any number $k$ of robots, $I(T, k)=\frac{2|T|}{k}$.

Proof. (Lemma 5.2.7) The upper bound follows from Lemma 5.2.2. We now concentrate on the lower bound. Indeed, by Lemma 5.2.6 the lower bound $I(T, k) \geq$ $\frac{2|T|}{k}-\frac{2 \epsilon}{k}$, is valid for any $k \geq k_{0}$, where $\epsilon, k_{0}$ are selected as specified in Lemma 5.2.6. So assume that $k \leq k_{0}$. Choose an integer $i$ sufficiently large such that $k \leq k_{0} \leq 2^{i} k$. Now observe that the following inequalities are valid

$$
\begin{aligned}
I(T, k) & =\frac{F_{T}(k)}{k} \text { (by definition) } \\
& \geq \frac{F_{T}\left(2^{i} k\right)}{k}(\text { by Lemma } 5.2 .4) \\
& =\frac{F_{T}\left(2^{i} k\right)}{2^{i} k} \cdot \frac{2^{i} k}{k} \\
& =I\left(T, 2^{i} k\right) \cdot \frac{2^{i} k}{k} \text { (by definition) } \\
& \geq\left(\frac{2|T|}{2^{i} k}-\frac{2 \epsilon}{2^{i} k}\right) \cdot \frac{2^{i} k}{k} \text { (by Lemma 5.2.6) } \\
& =\frac{2|T|}{k}-\frac{2 \epsilon}{k} .
\end{aligned}
$$

The last inequality is valid for any integer $k$ and any real number $\epsilon>0$. By letting $\epsilon \rightarrow 0$ the proof of the lemma is complete.

We are now in a position to prove the main theorem (Theorem 5.2.1) which was given at the beginning of the paper. 
Proof. (Theorem 5.2.1) Without loss of generality we may assume that the tree is a $d$-caterpillar, for some $d \geq 2$ (in fact, every tree is a $d$-caterpillar, for some $d \geq 2$, provided $d$ is sufficiently large). Now the proof of Identity (5.2) proceeds by induction on $d$. Recall that Lemma 5.2.7 is precisely the base case $d=1$. Suppose the identity in the theorem is valid for $d-1$. By Lemma 5.2.5 the subtree $T^{\prime}$ is obtained from $T$ by removing all its leaves is a $(d-1)$-caterpillar. Clearly, Identity (5.2) is valid for $\left|T^{\prime}\right|$, namely $I\left(T^{\prime}, k\right)=\frac{2\left|T^{\prime}\right|}{k}$. Therefore by repeating the proof of Lemma 5.2.6 we can show that for any real number $\epsilon>0$ there is a sufficiently large integer $k_{0}$ such that $I(T, k) \geq \frac{2|T|}{k}-\frac{2 \epsilon}{k}$, for all $k \geq k_{0}$. In turn, using this last statement we repeat the proof of Lemma 5.2.7 to prove the desired identity. This completes the proof of Theorem 5.2.1.

\subsubsection{Patrolling of Graph Trees}

Unlike geometric trees, where a robot during patrolling may change direction at any location in the graph that is either an interior point on an edge or a vertex, in graph trees a robot may change direction only on vertices of the tree. The corresponding idle time on such a graph tree $T$ for $k$ robots is denoted by $I^{\prime}(T, k)$.

Next we provide a patrolling algorithm for the case when all the edges have integer lengths (not necessarily the same). Let $e_{1}, e_{2}, \ldots, e_{2(n-1)}$ be the sequence of edges in the order they occur in a given preorder traversal. Define a partition from a sequence of integers as follows.

1. Let $\sigma: r_{0}=0<r_{1}<\cdots<r_{k}=2(n-1)$ be a sequence of integers and define sets of edges as follows: $E_{i}^{\sigma}:=\left\{e_{r_{i}+1}, e_{r_{i}+2}, \ldots, e_{r_{i+1}}\right\}$, where $i+1<k$; (Thus, each $E_{i}^{\sigma}$ is a sequence of consecutive edges in the preorder traversal, i.e, $E_{i}^{\sigma}$ 
represents a partitioning of the tree edges where every edge is assumed to be replaced by two directed edges.)

2. For each $i$, let $\left|E_{i}^{\sigma}\right|:=\sum_{e \in E_{i}^{\sigma}}|e|$;

3. Define $\Delta^{\sigma}:=\max _{i \neq j}|| E_{i}^{\sigma}|-| E_{j}^{\sigma}||$.

In fact, $\Delta^{\sigma}$ is the maximum difference among the partitioning of the tree (in terms of sum of the edges of each partition.)

4. Now select a sequence $\sigma_{0}: r_{0}=0<r_{1}<\cdots<r_{k}=2(n-1)$ which provides the most balanced partition of the preorder traversal in the sense that it attains

$$
\Delta:=\min _{\sigma} \Delta^{\sigma}=\min _{\sigma} \max _{i \neq j}|| E_{i}^{\sigma}|-| E_{j}^{\sigma} \| .
$$

(Note that there could be more than one such sequence.) From now on when writing $E_{i}$ without a superscript we will be referring to $E_{i}^{\sigma_{0}}$, where $\sigma_{0}$ was defined in Item 4 above. In fact, we need to find the most balanced partitioning of the tree to minimize $\Delta^{\sigma}$.

We now define a patrolling strategy that we will analyze in detail in the sequel.

Definition 3 (Eulerian Strategy $\mathcal{E S}$ ) For $i=1,2, \ldots, k$ the $i$-th robot is initially placed at the first vertex of the sequence of edges determined by the set of edges $E_{i}$. The robots move forever with speed 1.

Theorem 5.2.8 Consider a graph tree $T$ with $n$ nodes and $n-1$ edges such that all the edges have integer lengths. For any $k$ robots: 
1. A lower bound on any patrolling strategy is $\left\lceil\frac{2|T|}{k}\right\rceil$.

2. The idle time of the Eulerian patrolling strategy $\mathcal{E S}$ on the tree $T$ satisfies $I_{\mathcal{E S}}^{\prime}(T, k) \leq$ $\left\lceil\frac{2|T|}{k}\right\rceil+\Delta$.

3. If $\Delta=0$ then $I_{\mathcal{E S}}^{\prime}(T, k)=\left\lceil\frac{2|T|}{k}\right\rceil$.

Proof. We prove the three parts of the theorem separately.

Proof of Part 1: The robots start their movement at time $t=0$. Since the robots have speeds equal to 1 and the edges are of integer lengths, the vertices of the tree are visited by the robots at integer times. We can prove the following claim.

Claim $1 I^{\prime}(T, k)$ is an integer.

Proof. Consider a patrolling algorithm, say $\mathcal{S}$, which achieves the optimal idle time, i.e $I^{\prime}(T, k)=I_{\mathcal{S}}(T, k)$. The robots are initially placed on vertices of the tree and start patrolling at time $t=0$. Thus, vertices of the graph can only be visited at integer times. Now consider an edge of the tree with vertices $u, v$. View the edge as a closed interval $[u, v]$ with endpoints $u, v$ and integer length $s$.

It is clear that interior points $p$ of the edge $[u, v]$ will have idle time of the form $\ell+x(p)$ or $\ell^{\prime}+(s-x(p))$, where $x(p)$ is the distance of $p$ from $u$ and $s-x(p)$ the distance of $p$ from $v$ and $\ell, \ell^{\prime}$ are integers. Since the idle time is the supremum of the idle times over all points $p$ in the edge $[u, v]$, this value will be an integer. This proves the claim.

As a consequence of the Claim, we see that the optimal idle time must be an integer. It follows from Theorem 5.2.1 that $I^{\prime}(T, k) \geq\left\lceil\frac{2|T|}{k}\right\rceil$.

Proof of Part 2: It is easy to see that the idle time of the Eulerian Strategy satisfies $I_{\mathcal{E S}}(T, k) \leq \max _{i}\left|E_{i}\right|=\left|E_{\max }\right|$, where $E_{\max }$ is the set with maximum $\left|E_{i}\right|$. Also 
observe that

$$
2|T|=\sum_{i=1}^{k}\left|E_{i}\right| \geq k\left|E_{\min }\right|
$$

where among the sets $E_{i}$ we consider the set $E_{\min }$ which attains the value $\min _{1 \leq i \leq k}\left|E_{i}\right|$. It follows that

$$
\begin{aligned}
I_{\mathcal{E S}}(T, k) & \leq\left|E_{\max }\right|(\text { by definition of } I(T, k)) \\
& \leq\left|E_{\min }\right|+\Delta\left(\text { since }\left|E_{\max }\right|-\left|E_{\min }\right| \leq \Delta\right) \\
& \leq\left\lceil\frac{2|T|}{k} \mid+\Delta(\text { by Inequality }(5.5))\right.
\end{aligned}
$$

Proof of Part 3: This is immediate from Part 2 since $\Delta=0$. This completes the proof of the theorem.

When $T$ is a line graph and $k=2$, then the two robots can be placed to start at the endpoints of the line. Therefore, in this case $\Delta=0$ and regular cyclic patrolling strategy provides an optimal algorithm even for arbitrary integer length of the edge. However, in case where all the edges have integer lengths (not necessarily the same) the robots may not be able to attain the optimal idle time $I(T, k)=\frac{2|T|}{k}$ (for geometric trees) if they all start on vertices of the tree. For example, consider three robots on a line $T$ with three vertices and two edges, one edge of length 1 and the other of length $m-1$. For the geometric line we have that $I(T, 3)=\frac{2 m}{3}$. However, it is easy to see that for the line graph $I^{\prime}(T, 3)=m-1$.

In conclusion, for graph trees where all the edges have the same lengths, the cyclic strategy achieves the optimal idle time $I^{\prime}(T, k)=\left\lceil\frac{2|T|}{k}\right\rceil$. On the other hand, for the case where the edges have different lengths, Eulerian strategy achieves the 
idle time $I_{\mathcal{E} \mathcal{S}}^{\prime}(T, k) \leq\left\lceil\frac{2|T|}{k}\right\rceil+\Delta$, assuming we obtained the most balanced partitioning of the tree. It is clear that in this case the idle is not necessarily optimal, unless the partitioning leads to $\Delta=0$.

\subsection{On-Line Patrolling Algorithm with Rotor-Router}

All patrolling strategies proposed in Section 5.2 are centralized algorithms in that the robots receive instructions from a central controller. In this section we describe a distributed mechanism, so-called rotor-router, which has been extensively studied in the literature as a deterministic alternative to "random walk". Besides being distributed, the rotor-router is an on-line algorithm (i.e., the environment to be patrolled is unknown), which makes it a practical tool for patrolling an unknown environment as well.

The basic idea of rotor-router is to set locally shared memories at the nodes of the graph. Subsequently, the robots will be updating these shared memories as they visit the vertices of the graph. For example, the single rotor-router algorithm described in [21] is illustrated in Algorithm 5.1. Let $u$ be a vertex of the tree and denote by $d(u)$ the degree of $u$. Label the edges adjacent to $u$ with the numbers $0,1, \ldots, d(u)-1$. At each vertex $u$, there is a pointer, denoted by $\operatorname{exitport}(u)$, indicating the edge (adjacent to $u$ ) that should be traversed by the next agent visiting $u$. A robot visiting $u$, updates exitport ( $u$ ) before leaving $u$.

Algorithm 5.2 describes parallel rotor-routers for $k$ robots, which basically works in the same way as the single rotor-router. However, the only notable issue is when more than one robot is visiting node $u$ at the same time (step). In this case, 


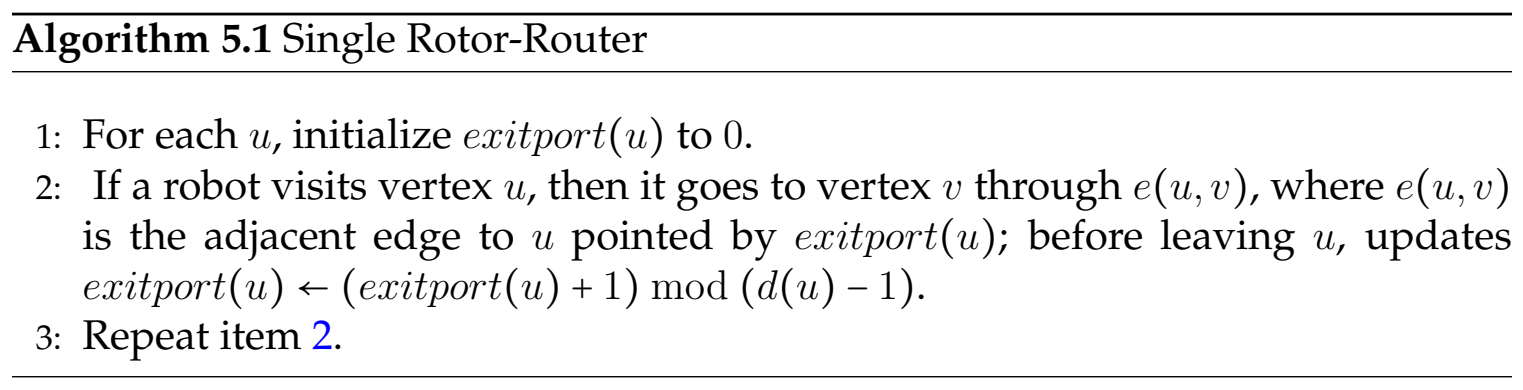

robots access exitport( $u)$ in a "mutual exclusion" way, that is robots access and update exitport $(u)$ one by one. Since all robots are identical, the order in which they access and update exitport $(u)$ does not affect the algorithm's performance.

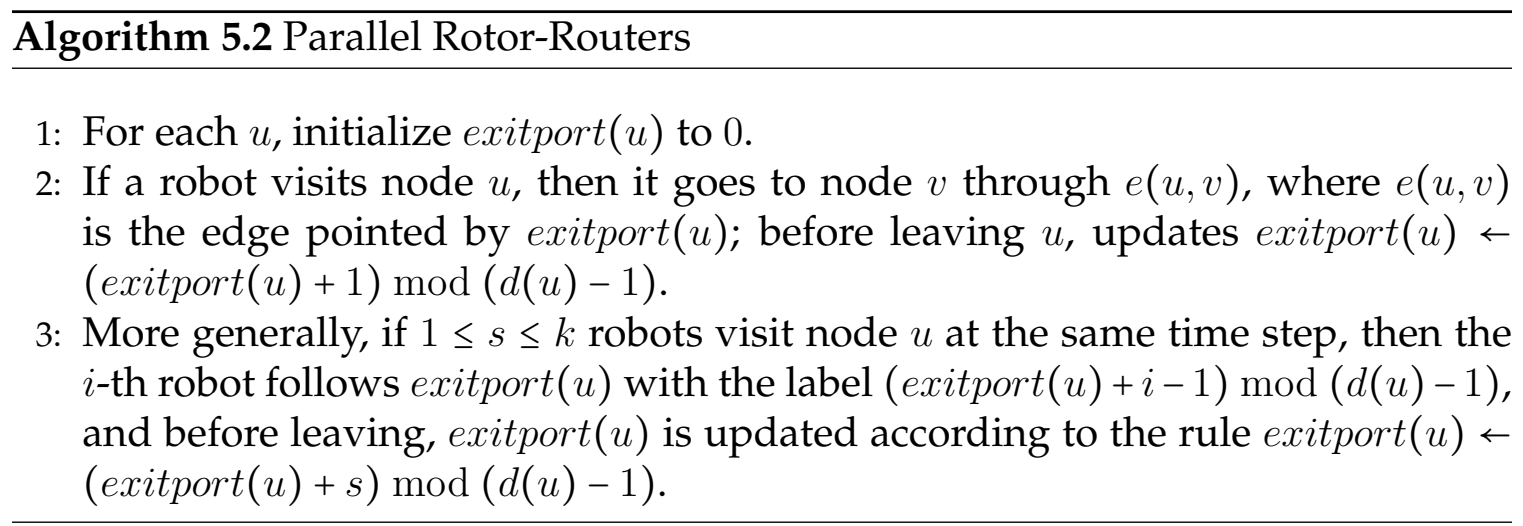

Before going further, we precisely define some of the most frequent concepts concerning the rotor-router algorithm used in the following sections.

Ports' pointers. Ports' pointers of node $u$, denoted by portspoint $(u)$, are local port numbers $0,1, \ldots, \operatorname{deg}(u)-1$ assigned to the adjacent edges of node $u$.

Exit port. Exit port at node u, denoted by exitport( $u$ ), keeps the port number of the edge (adjacent to $u$ ) that should be traversed by the next agent visiting node $u$. The exit ports are in fact the shared memories used by the robots in the rotor-router algorithm.

Robots' locations. Robots' locations at time step $t$, denoted by RobotsLoc(t), are 
the nodes where the robots are located at the end of time step $t$.

Initial configuration. Initial configuration of the (rotor-router) system, denoted by $I C$, is defined by ports' pointers assignment (for all nodes), the exit ports initialization (for all nodes), and the robots' initial locations at the beginning of the rotor-router algorithm, i.e, $t=0$.

System state. System state at time step $t$, denoted by $S_{t}$, is defined by the exit ports (at all nodes), and the robots' locations at time step $t$.

Stable state. Stable state is a system state that will repeat in future.

Since rotor-router is a deterministic model, independently from the initial configuration, after some transient time steps, the system reaches a stable state, in which the robots' locations and the exit ports repeats periodically. This behavior of rotor-router has been called periodic behavior of the system (see [21]). Interestingly, Yanovski et al. [96] showed that for a single robot the periodicity, i.e., the number of time steps until a stable state replicates, is only $2 m$ ( $m$ is the number of the edges). In regards of parallel rotor-routers, Chalopin et al. [21] showed that after stabilization, the graph is decomposed into some disjoint sub-cycles, where each of them is traversed independently by a sub-set of robots. This behavior of rotor-router has been called sub-cycle decomposition.

\subsubsection{Lower Bounds}

We know that the optimal algorithm for patrolling a graph tree of $n$ nodes, and

edges with equal lengths, satisfies $I^{\prime}(T, k)=\left\lceil\frac{2(n-1)}{k}\right\rceil$. The main problem we are interested in is whether the parallel rotor-router algorithm is optimal for $k$ robots on a graph tree with $n$ nodes? In this subsection we prove a lower bound on the 
asymptotic ratio of the rotor-router on a graph tree having the form of a star with three branches. The precise theorem is as follows.

Theorem 5.3.1 There is a star graph with $n+1$ vertices and an assignment of ports' pointers such that the competitive ratio of the rotor-router algorithm for two robots is at least $\frac{4}{3}$.

Proof. Consider the star graph depicted in Figure 5.1. It has $n+1$ vertices such that one vertex is at the center and each of its three branches has $\frac{n}{3}$ vertices. Let the three leaves of the star be named $A, B, C$.

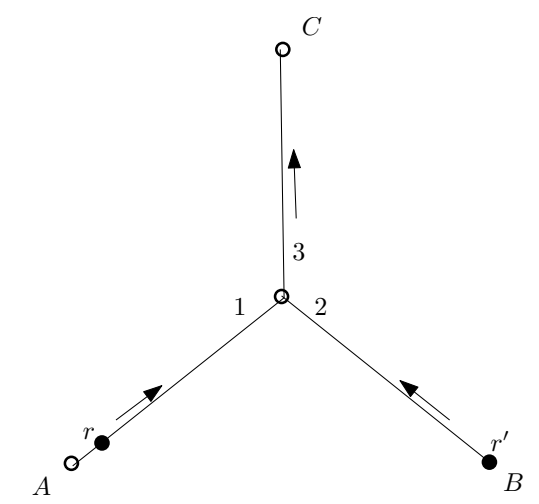

FIGURE 5.1: Proving a $4 / 3$ lower bound for patrolling the star graph by two robots $r, r^{\prime}$.

The ports on the vertices of the star are initially directed as follows. The ports' pointers at the central node are numbered 1,2,3 and oriented towards the leaves $A, B, C$, respectively. Further, the initial exit port at the center is 3 . The ports (both ports' pointers and exit ports) on all the vertices from the leaf $A$ (resp. $B$ ) to the center are oriented towards the center, while the ports (both ports' pointers and exit ports) on all the vertices from the leaf $C$ are oriented away from the center. 
First of all, observe that an optimal off-line schedule of the star graph for two robots is $\frac{2(n+1-1)}{2}=n$ as given by Theorem 5.2.1. We now propose the initialization of a rotor-router schedule and analyze its associated idle time.

Two robots $r, r^{\prime}$ start synchronously at the following two vertices of the graph. Robot $r$ starts at the vertex adjacent to the leaf $A$ while robot $r^{\prime}$ starts at the leaf $B$. The robots move at the same speed. The first robot to reach the center is $r$ which follows port 3 towards leaf $C$. When robot $r^{\prime}$ reaches the center a single time unit later it will be directed towards leaf $A$. Robot $r^{\prime}$ will follow port 1 at the center and head towards leaf $A$. Observe now that when robot $r^{\prime}$ is at $A$ robot $r$ is a vertex away from the leaf at $C$. Thus the two robots are in a periodic repetition of the schedules after time exactly $\frac{2 n}{3}$. It is easy to see that in the next periodic iteration of the rotor-router, robot $r$ will head towards leaf $B$ while robot $r^{\prime}$ towards leaf $A$. It follows that the idle time is exactly $\frac{4 n}{3}$. This gives rise to the $\frac{4}{3}$ competitive ratio of the rotor-router and completes the proof of the theorem.

In conclusion, we note that we could easily convert the star graph to a more general tree where each of the three "branches" of the star is replaced by a subtree attached to the center having the sum of lengths of all its edges equals to $\frac{n}{3}$. We do not know whether the competitive ratio of $\frac{4}{3}$ is the best possible value. In fact, it has been theoretically proved that rotor-router achieves the idle time $c I(T)$ for trees, where $c$ is at most 6 , and $I(T)$ is the optimal idle time of the centralized algorithm for trees [63]. However, we experimentally show that in most cases the rotor-router's idle time is optimal (in some cases it is off by 1), and there are only a few cases where the rotor-router's idle time is at most $\frac{4}{3}$ of the optimal idle time (of the centralized off-line algorithm). In other words, $c=4 / 3$ may be the least 
possible value for the competitive ratio (i.e., $\frac{4}{3} \leq c \leq 6$, where $c=6$ has been proved theoretically, while $c=\frac{4}{3}$ has been shown experimentally.

\subsubsection{Experimental Results}

In this section we provide experimental results based on the rotor-router model. We conducted experiments to compute cover time, lock-in time, and idle time, as well as periodicity for different random trees, star graphs, variable number of robots and initial configurations. For this purpose, we have implemented the rotor-router simulator in Java. The details of this simulator will be explained in Chapter 6. In order to run experiments, the simulator applied an algorithm (developed independently by David Aldous [5] and Andrei Broder [16]) to generate uniform random trees. Moreover, it uses a random number generator to generate uniform random initial configurations. In the following sub-sections, the results of the experiments are demonstrated by diagrams, but the detailed tables concerning the results of each experiment are added in the appendices.

\section{Random Trees}

For a given tree $T$ and a set of $k$ robots, in addition to the number of nodes and robots that affect the performance of rotor-router (measured by cover time, lockin time, and idle time, as well as periodicity), there are also some non-quantitative factors that may affect the system's performance. These non-quantitative factors are, the ratio of $m$ (number of edges) and $k$, initial configuration of the system, and tree structure. Therefore, we have run experiments on the rotor-router simulator to see 
how these factors may affect the performance of rotor-router on trees (all experimental designs and statistical computations of results are based on [57] Parts III and IV). We only considered trees in our experiments since we have obtained the optimal centralized algorithm for tree patrolling (see Section 5.2). In addition, it has been theoretically proved that rotor-router achieves $c . I(T)$ idle time for trees, where $c=6$, and $I(T)$ is the optimal idle time of the centralized algorithm [63].

In order to observe how the ratio of $m$ (i.e., $m=n-1$ for trees) and $k$ may affect the rotor-router performance, we considered settings for $k=1, \ldots, n$ robots for a given tree $T$ with $n$ nodes in all experiments. In order to see how various initial configurations may change the rotor-router performance, we have run experiments for 100 random initial configurations for each set of $k=1, \ldots, 50$ robots on a random tree $T$ with 50 nodes, i.e, 5,000 experiments in total. All relevant plots for these experiments are illustrated in Figure 5.2. Afterwards, in order to study the effect of tree structure, we ran experiments for 50 different random trees (each of them with 50 nodes), and 100 random initial configurations for each set of $k=1,2, \ldots, 50$ robots on each tree, i.e., 250,000 experiments in total. All respective plots for these experiments are displayed in Figure 5.3. In all displayed diagrams in Figures 5.2 and 5.3, the red, green, and blue plots represent the maximum, minimum, and average times respectively. The black vertical lines demonstrates the $95 \%$ confidence intervals for the cases of average time.

Cover Time. Cover time is the number of time steps until each node of the graph has been visited by at least one robot, regardless of the initial configuration of the system. Diagrams in Figures 5.2( $a$ ) and 5.3( $a$ ) show the cover time of rotor-router 

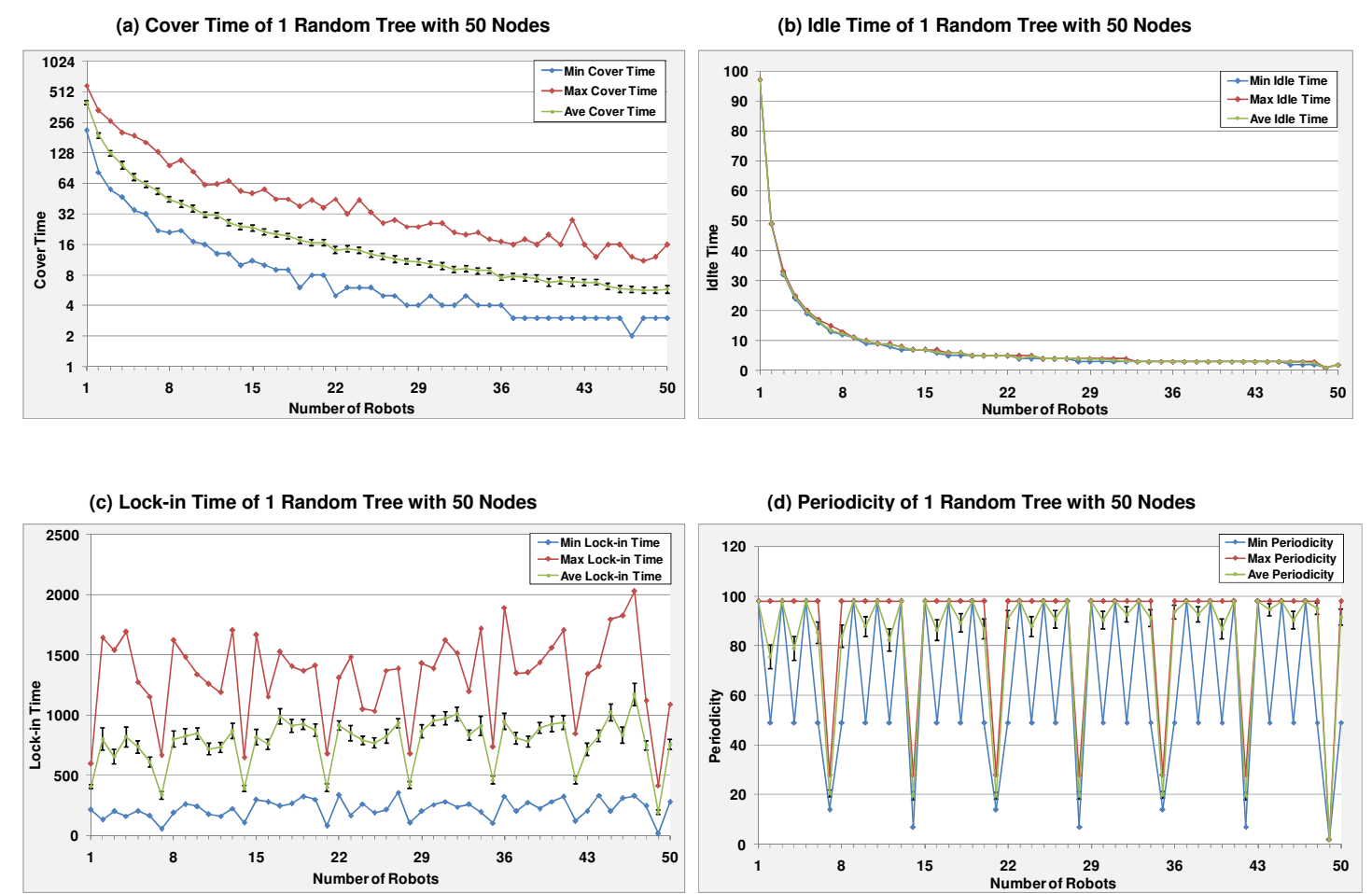

FIGURE 5.2: (a) cover time, (b) idle time, (c) lock-in time, and (d) periodicity, of rotor-router algorithm for a random tree with 50 nodes, and 100 distinctive initial configurations for each set of $k=1,2, \ldots, 50$ robots, drawn against the number of participating robots in each experiment.

algorithm for 100 distinctive initial configurations for each set of $k=1,2, \ldots, 50$ robots, running over 1 and 50 random trees respectively (all trees have 50 nodes).

Variant times on the red (maximum) and blue (minimum) plots on Diagram 5.2( $a$ ), show clearly how the initial configurations may affect the cover time. However, in Diagram 5.3(a) these variations may not be only due to variant initial configurations, but the different tree structures as well. Moreover, the noticeable difference 

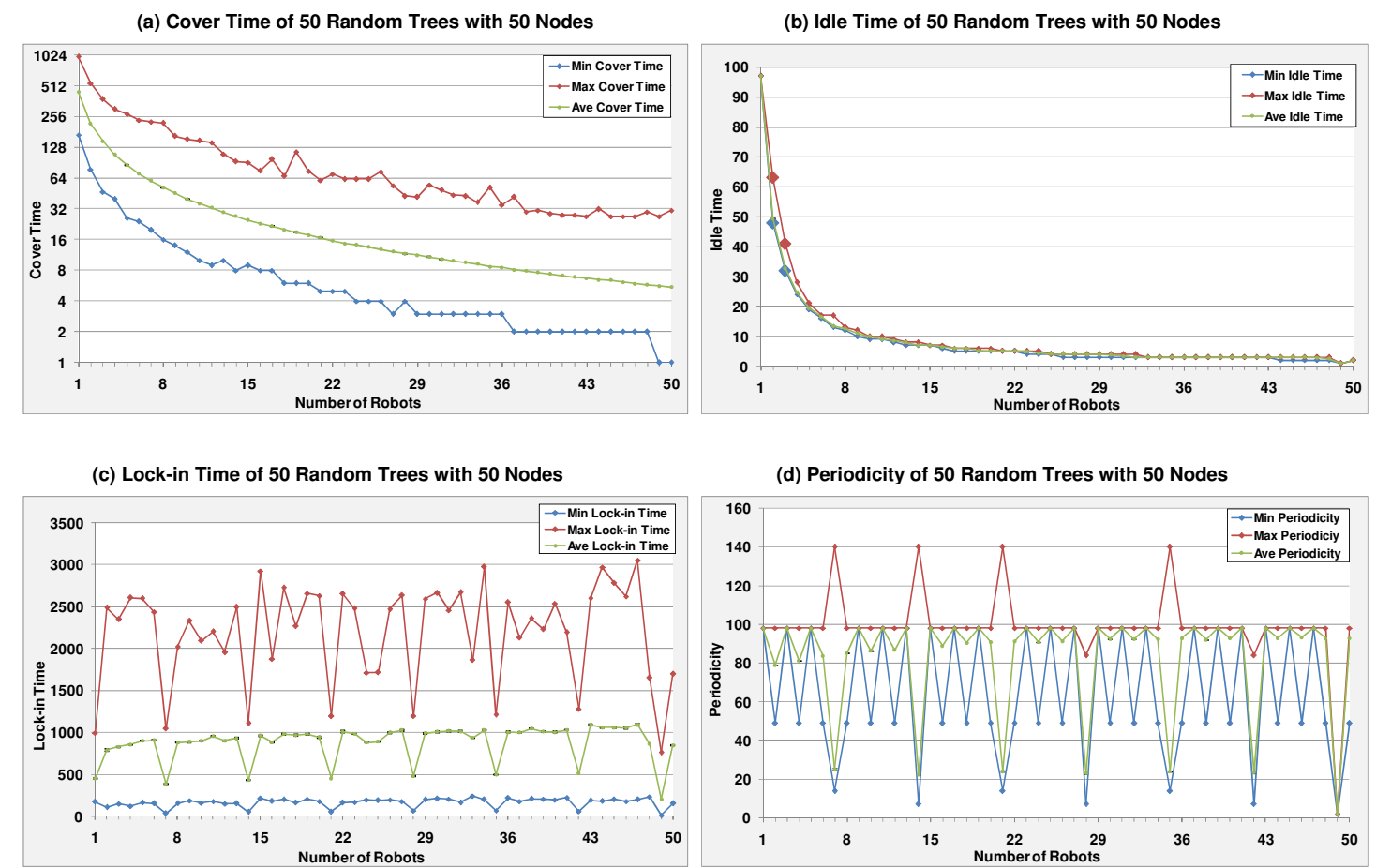

FIGURE 5.3: (a) cover time, (b) idle time, (c) lock-in time, and (d) periodicity of rotor-router algorithm on 50 random trees with 50 nodes, and 100 distinctive initial configurations for each set of $k=1,2, \ldots, 50$ robots, drawn against the number of participating robots in each experiment.

in values of plots (maximum, minimum, and average) on Diagrams 5.2(a) and 5.3( $a)$ is another proof of dependence of cover time on the tree structure. According to these diagrams, it seems that the ratio of $m$ and $k$ does not play a role for the case of cover time.

We may also notice that although adding more robots to the system improves (reduces) the cover time, at some point where $k>n / 2$ adding more robots does not 
change the cover time significantly. According to our results, even for the worse initial configurations and tree structures the cover time is $O(m D / k)$, where $D$ is the diameter of the tree.

Idle Time. Idle time is the number of time steps that an edge remains unvisited by any robots. The idle time of a tree $T$ is the maximum idle time over all its edges. To compute the idle time of tree $T$, we run rotor-router until the system stabilizes, when it will follow a periodic behavior afterward. Then, we run rotor-router for two more periodic cycles while keeping track of the visited edges. Thereafter, we compute the idle time of each edge, and then take the maximum one as the idle time of tree $T$.

Diagrams in Figures 5.2(b) and 5.3(b) display the idle time of rotor-router algorithm for 100 distinctive initial configurations for each set of $k=1,2, \ldots, 50$ robots running over 1 and 50 random trees respectively (all trees have 50 nodes). According to these diagrams, in most cases the idle time is almost $2 m / k$ (where $m=n-1$ ), which is optimal. However, there are a few cases (e.g., for $k=2,3$ in Diagram 5.3(b) where the data points are highlighted on the plots) where the idle time is slightly more than $2 m / k$ depending on the initial configuration of the system and structure of the tree (as we also discussed for the star tree in Section 5.3.1).

According to [21], after stabilization the system is decomposed into some disjoint sub-cycles. Apparently, the robots are also divided into subsets traversing each sub-cycle independently, while the number of robots in each sub-cycle is proportional to the number of edges in that sub-cycle. Consequently, the idle time remains almost constant in most cases regardless of the initial configurations and structure of the trees. Locating the red (maximum), blue (minimum) and green 
(average) plots closely on each other in Diagrams 5.2(b) and 5.3(b), confirms that the idle time is independent of the initial configurations and trees structure in most cases. However, apparently for some classes of trees (e.g., star graph), and number of robots (e.g. $k=2,3$ ) the initial configuration may lead the system to some sub-cycle decomposition where the number of robots and the edges in each subcycle are not balanced up, and consequently the idle time would not be optimal. According to our results only for $k=2,3$ the difference between maximum and minimum idle times is noticeable, where the maximum $I(T, k)$ is $\frac{4}{3}$ of minimum $I(T, k)$ (see Diagram 5.3(b)).

According to Diagrams 5.2(b) and 5.3(b), it seems that the ratio of $m$ and $k$ does not play a role for the case of idle time. It is also worthy to note that although adding more robots to the system improves (reduces) the idle time, at some point where $k>m / 2$ adding more robots does not change the idle time significantly.

Lock-in Time. Lock-in (stabilization) time is the number of time steps until the system reaches the first stable state, i.e., all the system states will periodically repeat after the lock-in time. Diagrams in Figures 5.2(c) and 5.3(c) show the lock-in time of the rotor-router algorithm for 100 distinctive initial configurations for each set of $k=1,2, \ldots, 50$ robots running over 1 and 50 random trees respectively (all trees have 50 nodes).

Digram 5.2(c) shows clearly that lock-in time depends on the initial configuration of the system since the red (maximum) and blue (minimum) plots display different values. However, on Diagram 5.3(c) these variations may be due to initial configurations as well as tree structures. Moreover, the variant values of the 
plots (maximum, minimum and average) on Diagrams 5.2(c) and 5.3(c) confirms the dependence of lock-in time on the tree structure as well.

The sinusoidal pattern of the lock-in time plots is a notable fact of Diagrams (c). According to these diagrams, it seems that the value of the greatest common divisor between $m$ and $k$ plays a significant role in the lock-in time. According to Diagrams (c), for data point where $m=49$ and $k$ are co-prime, we obtain the maximum lock-in times, while we have the minimum values when $m$ and $k$ have 7 as the greatest common divisor. Moreover, we may note that the lock-in time never goes beyond $O(m D)$ in our experiments.

Periodicity. Periodicity is the number of time steps between two consecutive stable states (after the system stabilizes). Diagrams in Figures 5.2(d) and 5.3( $d)$ display the periodicity of rotor-router for 100 distinctive initial configurations for each set of $k=1,2, \ldots, 50$ robots running over 1 and 50 random trees respectively (all trees have 50 nodes).

Variant values on the red (maximum) and blue (minimum) plots on Diagram 5.2(d) represents the dependence of periodicity on the initial configurations. However, these variations on Digram 5.3(d) may be due to initial configurations as well as tree structures. Moreover, the variant values of the plots (maximum, minimum, and average) on Diagrams 5.2( $d)$ and 5.3(d) show clearly the dependence of periodicity on the tree structure. Perhaps different initial configurations and tree structures lead the system to different sub-cycle decomposition and consequently different periodicity.

In addition to tree structure and initial configuration of the system, the value of the greatest common divisor between $m$ and $k$ plays a significant role in periodicity 
as well. This also justifies the sinusoidal pattern of the periodicity plots. We can observe on Diagrams (d), for the data points where $k$ and $m=49$ are co-prime, (e.g., for $k=1,3,5,9,11,13,19,23,25,27, \ldots)$ periodicity is $2 m$ apparently regardless of the initial configurations and tree structures. On the other hand, for the data points where $m$ and $k$ have 7 as the greatest common divisor (e.g., $k=7,14,21,28,35,42$ ) we observe that minimum and average periodicity is less than $m$. However, for the same data points $(k=7,14,21,28,35,42)$ on Digram 5.3(d) there are cases where the maximum periodicity is even more than $2 m$, which seems tree structure plays a bigger role in these cases. For other numbers of robots (e.g., $k=2,4,6,8,10, \ldots$ ) the periodicity is between $m$ and $2 m$ depending on the initial configurations and tree structure. Based on our experiments we may conclude that periodicity of rotorrouters is $O(m)$ for trees.

\section{Star Graphs}

In order to highlight the competitive ratio of the idle time clearly, we have run experiments for the star graphs named "Tripod" and "Clover" for the following settings:

1. We ran experiments for Tripod graph (Figure 5.1) with 31 nodes, 2 robots, and the same initial configuration as the one in the proof of Theorem 5.3.1. We obtained the idle time of 38 , which is almost $\frac{4}{3}(2 m / k)$, for this setting.

2. We ran experiments for Tripod graph with 31 nodes, and 2 robots in the starting positions as in the proof of Theorem 5.3.1, but for 100 distinctive random ports' pointers and exit ports. In total 100 experiments were conducted and 
maximum, minimum and average idle times of 38,30 , and 32 were obtained respectively.

3. We ran experiments for Tripod graph with 31 nodes, and 100 distinctive random initial configurations for each set of $k=1,2,3, \ldots, 30$ robots , i.e., 3, 000 experiments in total. We observed that the idle time was almost optimal for all cases except for $k=2$ where the maximum, minimum and average idle times were 39, 29 and 32 respectively over 100 random initial configurations.

4. First we ran experiments for Tripod graph with 106 nodes, and 100 random initial configurations for each set of $k=2,3, \ldots, 105$ robots, i.e., 10,500 experiments in total. Then, we replaced the tree branches of Tripod graph by random sub-trees and named the new graph "Clover". We ran experiments for Clover with 106 nodes, and 100 random initial configurations for each set of $k=2,3, \ldots, 105$ robots, i.e., 10, 500 experiments in total. The results of the experiments for these two types of star graphs are illustrated in Figures 5.4 and 5.5. As you may note, all the diagrams follow the same patterns as the diagrams in Figure 5.2 and 5.3. Likewise, the discussions we had for random trees apply for these star graphs.

In our experiments for Tripod and Clover, the maximum and minimum idle times were noticeably different for $k=2$ as we expected. For the Tripod graph the minimum and maximum idle times are receptively 105 (104 for Clover), and 139 (138 for Clover). This confirms the idea that for any graph similar to star graphs, which has one node in the center and three branches with length $\frac{n}{3}$ connected to 
it, the idle time may vary based on the initial configuration. However, as our experiments shows the idle time is $\frac{4}{3}(2 m / k)$ in the worse case.
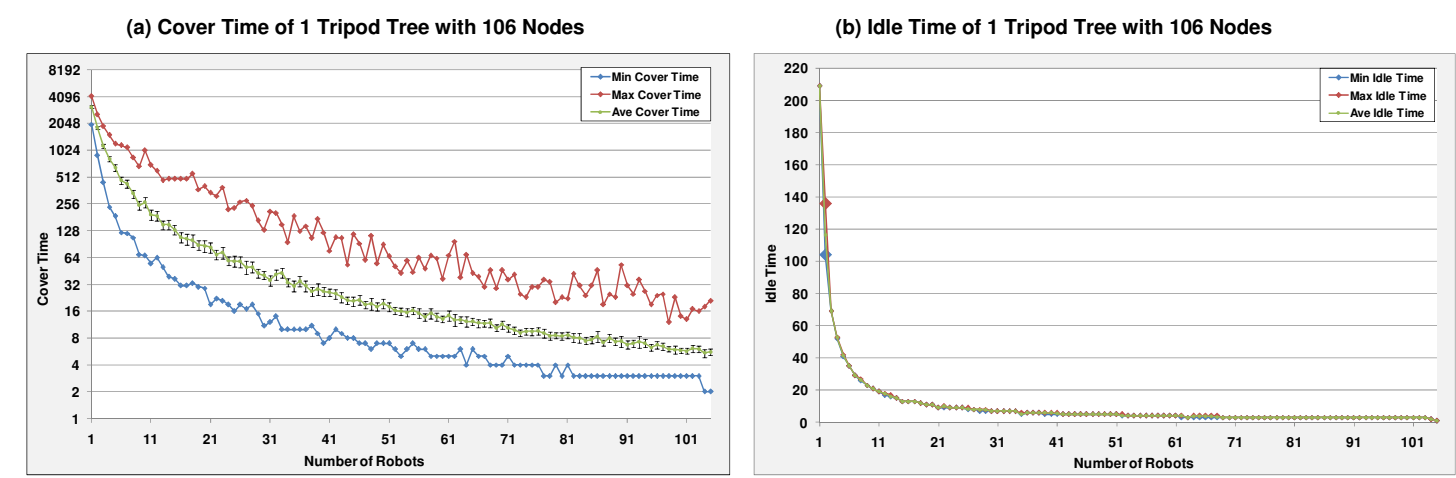

(c) Lock-in Time of 1 Tripod Tree with 106 Nodes
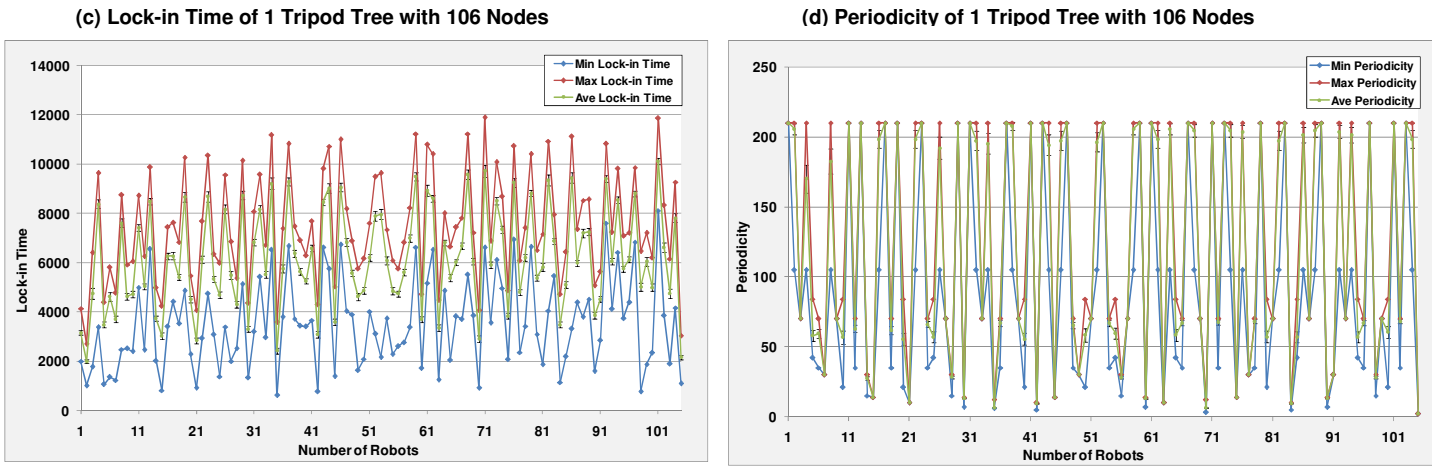

FIGURE 5.4: (a) cover time, (b) idle time, (c) lock-in time, and (d) periodicity of rotor-router algorithm for a Tripod tree with 106 nodes, and 100 random initial configurations for each set of $k=1,2, \ldots, 105$ robots, drawn against the number of participating robots.

\subsection{Conclusion and Open Problems}

In this chapter we investigated patrolling a connected tree $T$ by a team of $k$ mobile robots. We proposed an optimal off-line algorithm for $k$ patrolmen with the same 

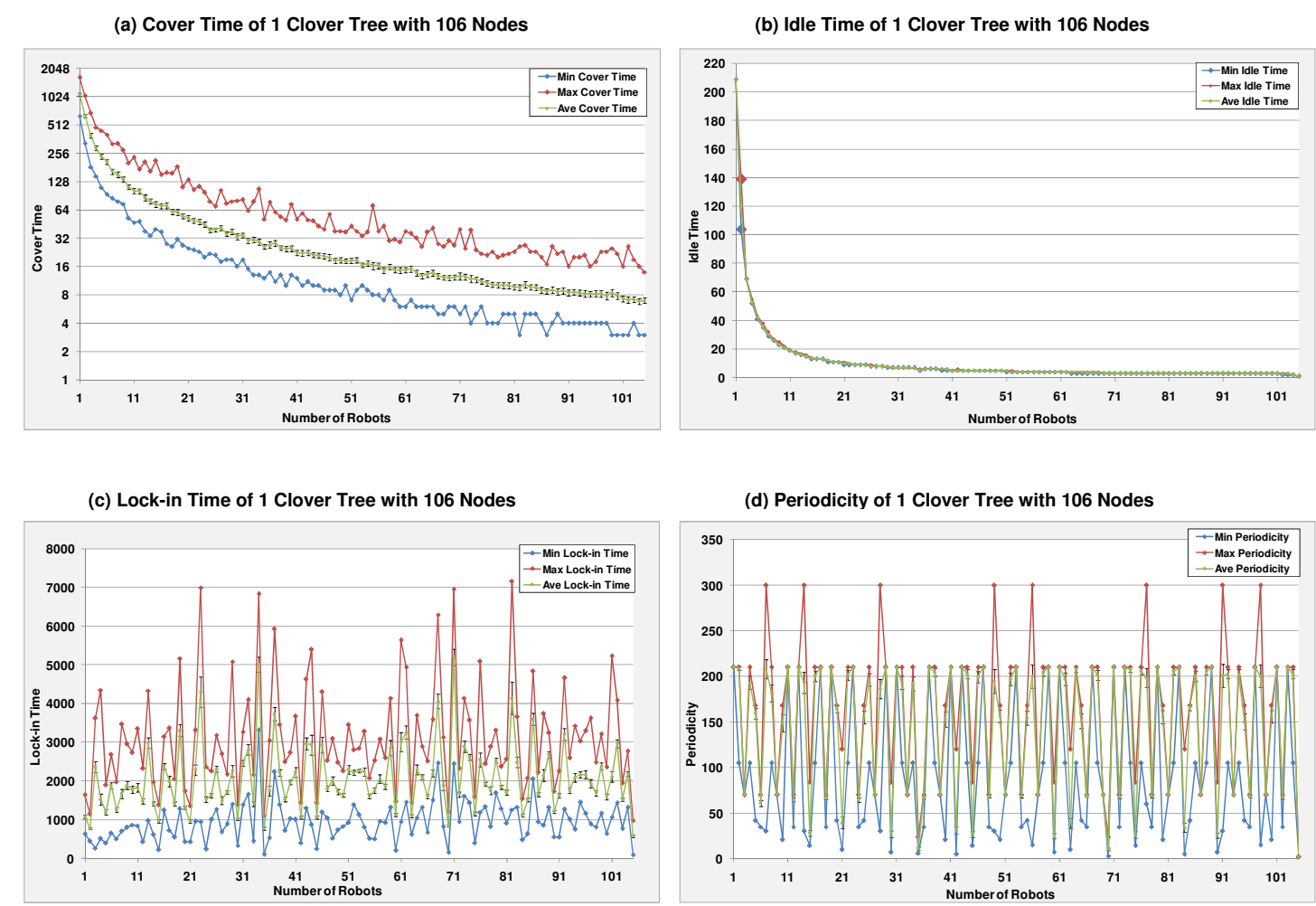

FIGURE 5.5: (a) cover time, (b) idle time, (c) lock-in time, and (d) periodicity of rotor-router algorithm for a random Clover tree with 106 nodes, and 100 random initial configurations for each set of $k=$ $1,2, \ldots, 105$ robots, drawn against the number of participating robots.

speed and zero visibility patrolling a geometric tree. We then extended our results to a graph tree. Since we obtained the optimal centralized patrolling algorithm for trees, we also studied distributed patrolling for trees considering the rotor-router mechanism. It has been theoretically proved that rotor-router achieves the idle time $c I(T)$ for trees, where $c$ is at most 6 , and $I(T)$ is the optimal idle time of the centralized algorithm for trees [63]. We experimentally showed that the resulting 
rotor-router has competitive ratio at least $4 / 3$. In other words, according to our experiments $c=4 / 3$ may be the least possible value.

Some open problems are investigating distributed patrolling for Eulerian or arbitrary graphs. It would be also interesting to investigate the rotor-router mechanism using heterogeneous robots, for instance, robots with different speeds and visibility or even faulty robots. Some other interesting open problems may be to study the off-line and on-line patrolling strategies for general graphs. 


\section{Chapter 6}

\section{Rotor-Router Simulator}

\subsection{Introduction}

In this chapter we discuss the details of rotor-router simulator implemented in Java. As we discussed in Chapter 5 (Section 5.3), to study the effects of initial configurations and tree structure on rotor-router performance, we need to run experiments because of the following reasons. First, for a given tree $T$ with $n$ nodes, and a team of $k$ robots, there are too many initial configurations to test. Secondly, for the given number of nodes $n$, there are many tree structures to consider. Finally, the behavior of parallel rotor-routers is very complicated, which makes it almost impossible to track without a computer program (to implement the algorithm), even for small numbers of $n$ and $k$.

\subsubsection{Preliminaries and Notation}

In order to explain the details of the rotor-router simulator, we first briefly provide some terminologies used in the simulation modeling (see [57] Part V for more details and examples), and then explain some frequently used notations in the next 
sections. Simulation is in fact a useful technique for analyzing the performance of computer system, when the system to be studied is not available, or is very complex. Every simulation applies a specific type of simulation model, which provides a tool to predict the performance or compare several alternatives of a system. As a simulation model, rotor-router is continuous-time, discrete-state (discrete-event), $d y$ namic, deterministic, nonlinear, and open. Consequently, rotor-router simulator is a discrete-state model simulation since it uses a discrete-state model of the system.

In a simulation model, the state variables are the variables whose values define the state of the system, and a change in the system state is called an event. In a continuous-time simulation model, the system states are defined at all times. However, if the state variables of the system states are discrete, the model is discrete-state (discrete-event). Notice that a discrete-event simulation may use continuous-time values. Moreover, the model is dynamic if time is a variable, and it is deterministic if its output can be predicted with certainty. In addition, the model is nonlinear if the output parameters are nonlinear function of the input parameters, and the model is open if the input parameters are independent and external to the model.

As discussed in Chapter 5, the basic idea of rotor-router is to set locally shared memories at the nodes of tree $T$. Then, the robots will be updating these shared memories as they visit the vertices of $T$ (see Section 5.3 for a detailed explanation of rotor-router algorithm). We denote by $V$ the set of verticies of $T$ such that $V=$ $\left\{v_{0}, v_{1}, \ldots, v_{n-1}\right\}$, and $|V|=n$. Let $v$ be a vertex of $T$, then $d(v)$ denotes the degree of $v$. We assume the ports' pointers at $v$, denoted by portspoint $(v)$, are initially labeled from $0,1, \ldots, \operatorname{deg}(v)-1$, i.e, portspoint $(v)=\{0,1, \ldots, \operatorname{deg}(v)-1\}$. The set of ports' pointers of all $v \in V$ are denoted by PortsPointers such that PortsPointers $=$ 
$\left\{\operatorname{portspoint}\left(v_{0}\right), \operatorname{portspoint}\left(v_{1}\right), \ldots, \operatorname{portspoint}\left(v_{n-1}\right)\right\}$. Exit port at $v$ is denoted by $\operatorname{exitport}(v)$, where $\operatorname{exitport}(v)=i$ and $i \in\{0,1, \ldots, \operatorname{deg}(v)-1\}$.

The state variables (whose values define the state of the system) of the rotorrouter model at time step $t$ are defined by the exit ports (at all the vertices), and robots' locations at time step $t$. Let ExitPorts $(t)$ denote the set of exit ports of all $v \in V$ at time step $t$, i.e., ExitPorts $(t)=\left\{\operatorname{exitport}\left(v_{0}\right), \operatorname{exitport}\left(v_{1}\right) \ldots, \operatorname{exitport}\left(v_{n-1}\right)\right\}$. Likewise, RobotsLoc(t) $=V^{\prime}$ denotes the robots' locations at time step $t$, where $V^{\prime} \subseteq V$ and $\left|V^{\prime}\right|=k$. Consequently, the system state at time step $t$ denoted by $S_{t}$ is defined by $S_{t}=\{\operatorname{RobotsLoc}(t)$, ExitPorts $(t)\}$. We denote $S_{0}$ as the initial state of the system, which is in fact the initial location of robots, and exit ports of all nodes at time step $t=0$, i.e., $S_{0}=\{\operatorname{RobotsLoc}(0), \operatorname{ExitPorts}(0)\}$.

As the performance criteria, the rotor-router simulator will compute the cover, lock-in, and idle time, as well as periodicity, respectively denoted by $C(T, I C)$, $L(T, I C), I(T, I C), P(T, I C)$, where $T$ denotes the tree in which the measurement parameters are evaluated considering the initial configuration $I C$. The initial configuration $I C$, is defined by the ports' pointers (of all nodes), and the system initial state, i.e., $I C=\left\{\right.$ PortsPointers,$\left.S_{0}\right\}$

Every simulator uses a random number generator (RNG), which is a physical or computational device to generate random numbers, in order to run experiments. Random numbers represent the value of a random variable uniformly distributed on $(0,1)$, which were originally generated by using different techniques. However, in modern approach, a computer (Pseudorandom number generator) is used to generate pseudorandom numbers. These pseudorandom numbers are in fact a sequence of deterministically generated values, but have the appearance of uniform $(0,1)$ 
random variables. One of the most common approach to generate pseudorandom number is to use an initial value called seed, and then compute the next values recursively (see [79] Chapter 3 for more details).

The rotor-router simulator uses random numbers in the process of generating random initial configurations, as well as tree structures. Java uses an instance of Random class to generate a stream of pseudorandom numbers. A method of this class, called nextInt(int $n$ ), returns an integer value between 0 (inclusive) and the specified value $n$ (exclusive). In the following procedures, we assume $\operatorname{Random}(n)$ is a function to generate a random integer number on $[0, n)$.

\subsection{Design of Rotor-Router Simulator}

A snapshot of the rotor-router simulator is demonstrated in Figure 6.1. This simulator has been designed in such a way that by choosing the appropriate parameters (“Number of Nodes”, “Number of Graphs”, “Max Number of Robots”, “Robots Factor", and "Number of Initial Configurations"), the user is able to run as many experiments as she/he is willing to instantly. For instance, in Figure 6.1, the user has selected to run experiments for 10 random trees with 50 nodes, and 100 distinctive initial configurations for each set of $k=1,2, \ldots, 50$ robots (since "Max Number

of Robots", and "Robots Factor" are receptively set to 50 and 1). Considering these settings, 50,000 experiments will be run in total, and the results will be saved into text files that can be used for further analysis (for instance, see the files attached in the appendices for the experiments ran in Chapter 5, Section 5.3 ).

Algorithm 6.1 illustrates the structure of the rotor-router simulator. The input 


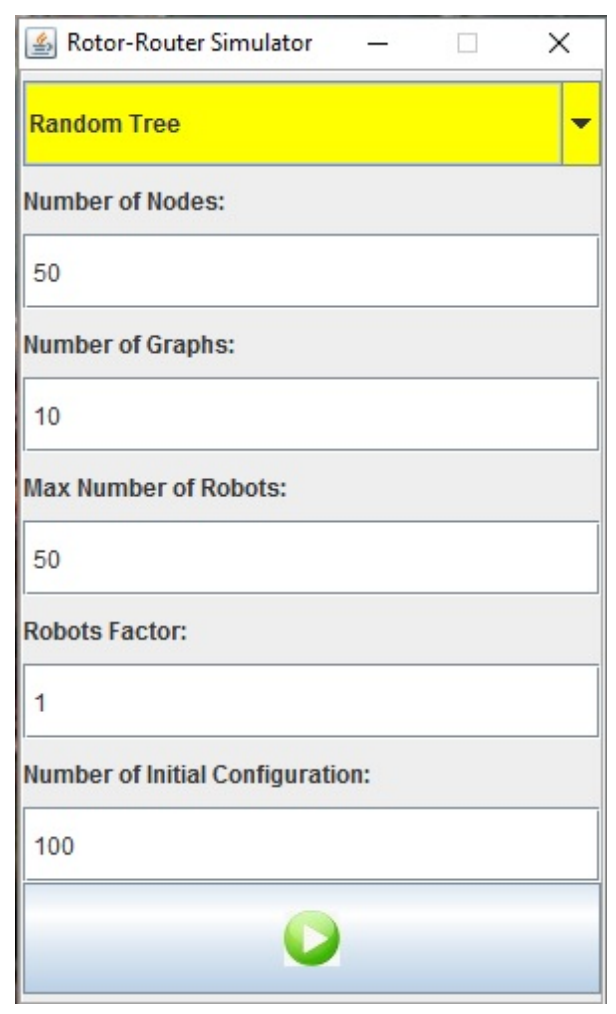

FIGURE 6.1: A snapshot of rotor-router simulator

parameters of the simulator are denoted by $n, \alpha, N u m T, N u m K$, and NumIC, which respectively represent "Number of Nodes", "Robots Factor", "Number of Graphs", "Max Number of Robots", and "Number of Initial Configurations". According to Algorithm 6.1, given the number of nodes $n$, the simulator generates a random tree $T$ by calling Algorithm 6.3. Then considering $T$, the number of robots is set to 1, i.e., $k=1$, and Algorithm 6.4 is called to generate a random initial configuration $I C$, for the given tree $T$ and $k$ robots. Afterwards, Algorithm 6.5 is called to apply the rotor-router algorithm on $T$ with initial configuration $I C$. As Algorithm 6.5 is running, the cover time $C(T, I C)$ is detected at some point, and finally the algorithm terminates when the system stabilizes. As the result of stabilization, 


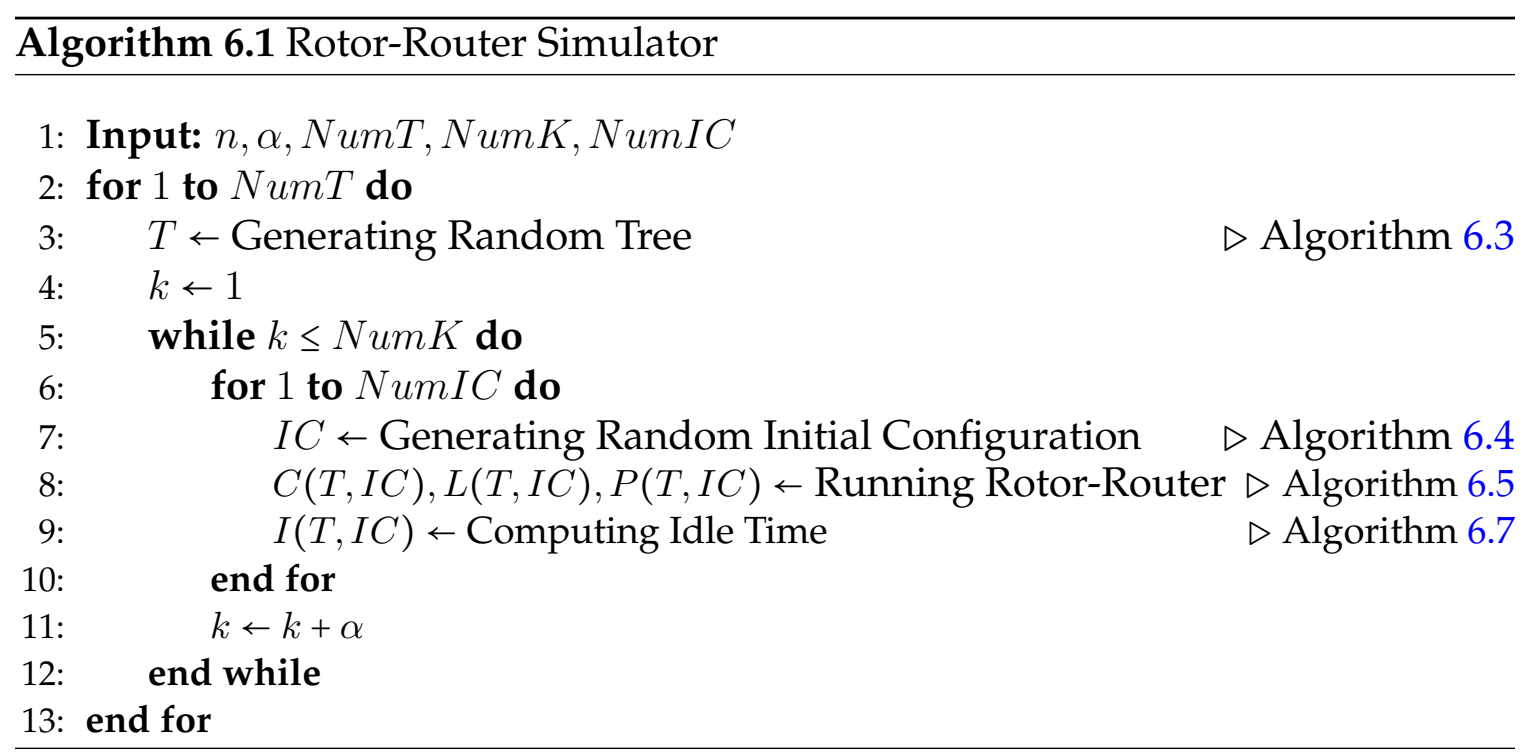

the lock-in time $L(T, I C)$ and periodicity $P(T, I C)$ are detected as well. Then, Algorithm 6.7 is called to compute the idle time $I(T, I C)$ for the tree $T$ and initial configuration $I C$.

After computing the idle time $I(T, I C)$ for $T$ with initial configuration $I C$, the simulator generates a new initial configuration, say $(I C)^{\prime}$, and runs rotor-router on $T$ considering $(I C)^{\prime}$. Once all NumIC initial configurations are generated for $k=1$, the number of robots is increased by $\alpha$, and rotor-router will be run on $T$ for new set of $k+\alpha$ robots for all NumIC initial configurations. Once all NumK number of robots are considered, a new random tree, say $T^{\prime}$, is generated and the whole process will be repeated for $T^{\prime}$. The simulator terminates once all NumT random trees are generated an tested. All the above mentioned algorithms will be explained in more details in the following sub sections. 


\subsection{Generating Uniform Random Trees}

In order to generate uniform random trees, i.e., with a given number of nodes $n$, every tree has the same chance to be generated, we consider the "Aldous-Broder algorithm" (developed independently by David Aldous [5] and Andrei Broder [16]). Initially, this algorithm was developed to generate a uniform random spanning tree of graph $G$. A spanning tree of graph $G$, is a subgraph that is a tree which includes all vertices of $G$. In fact, $G$ may have several spanning trees, and the one chosen randomly from among all the spanning trees with equal probability is called a uniform random spanning tree. Algorithm 6.2, illustrates the original Aldous-Broder algorithm.

Assuming $G$ is a complete graph (every vertex is connected to all other vertices), the rotor-router simulator applies Algorithm 6.3, which is based on the AldousBroder algorithm, to generate a uniform random tree $T$ with a given number of nodes $n$. According to this algorithm, a vertex $u$ is first selected randomly among all the vertices of $G$. Then, a new vertex $v$ is selected randomly, and the connecting edge $e(u, v)$ from $u$ to $v$ is traversed. If $v$ has not been visited before, $e(u, v)$ is added as one of the edges of spanning tree $T$. This process will be repeated until all vertices of $G$ have been visited.

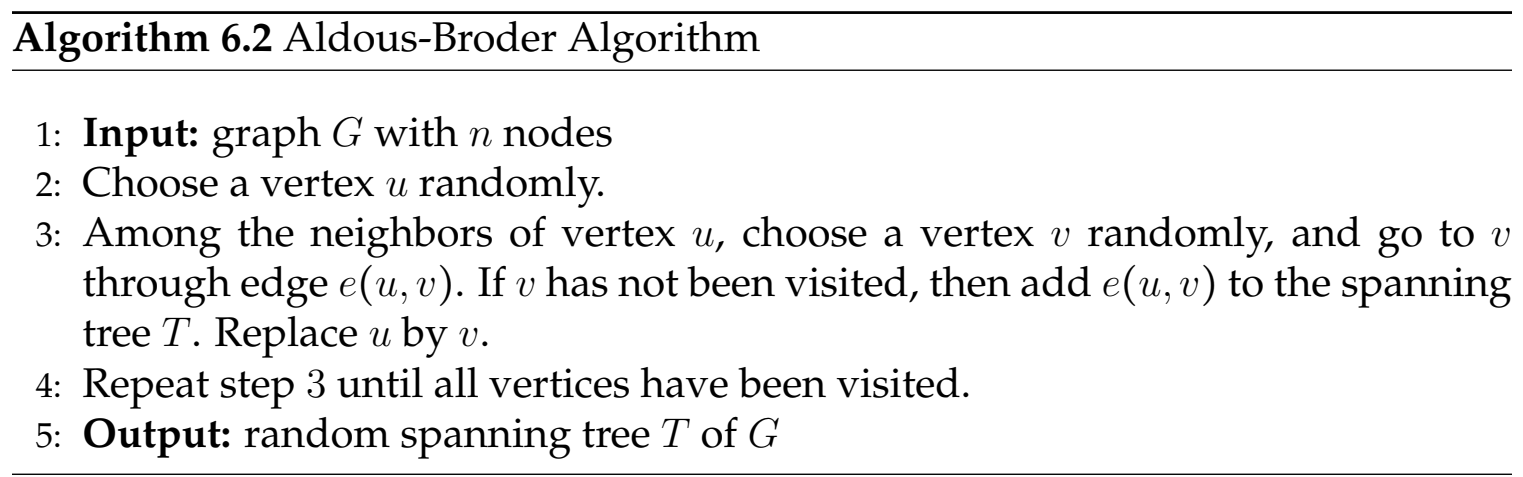




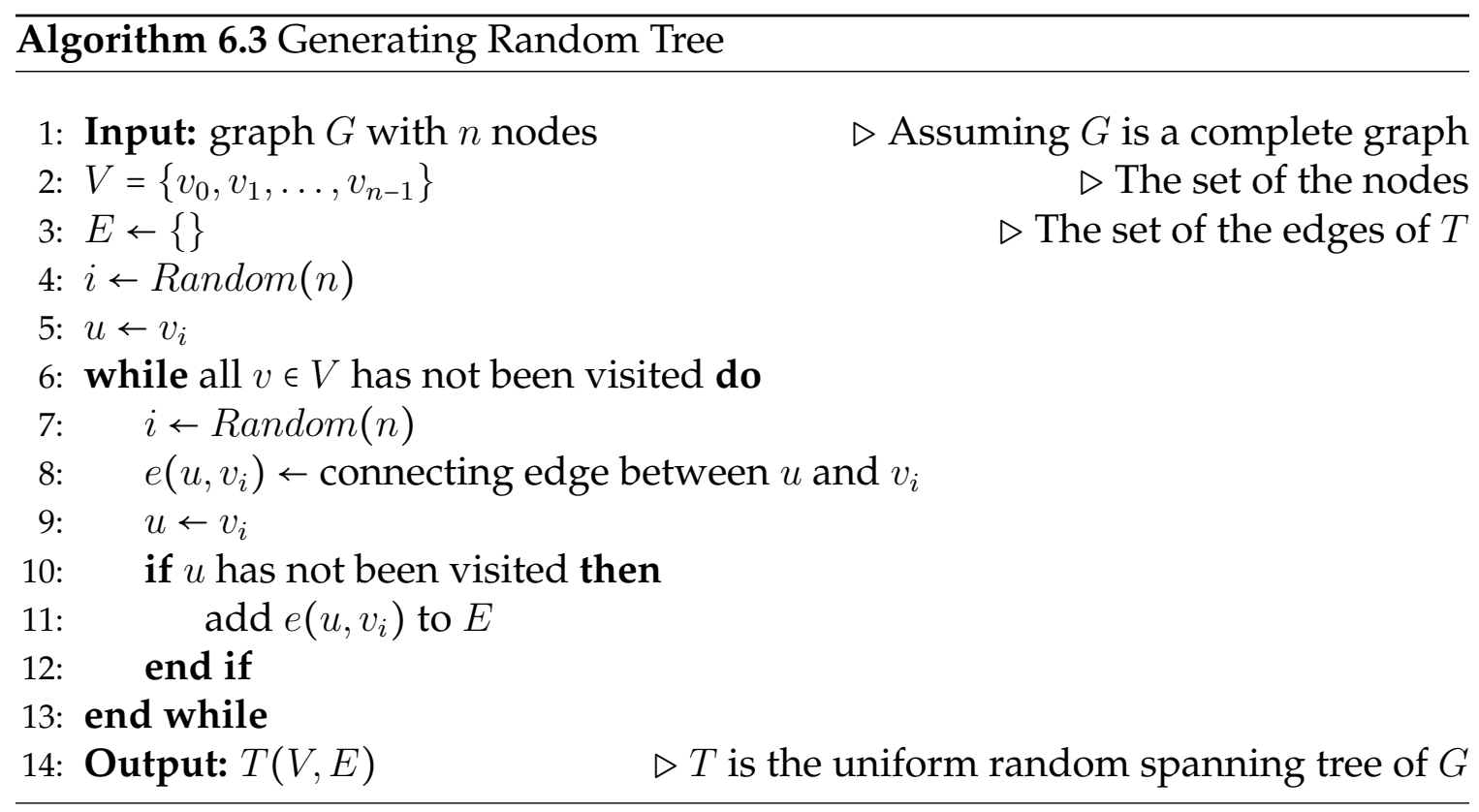

\subsection{Generating Uniform Random Initial Configurations}

The rotor-router simulator employs Algorithm 6.4 to generate a uniform random initial configuration $I C$, for given $T$ and $k$ robots. The initial configuration is defined by ports' pointers, and initial exit ports of all nodes, as well as initial locations of robots. In order to generate a random initial configuration, each of these parameters are generated randomly as follows.

Random Ports' Pointers. Considering portspoint $(v)=\{0,1, \ldots, \operatorname{deg}(v)-1\}$ as the initial ports' pointers of $v$, there are $\operatorname{deg}(v)$ ! permutations to choose randomly a sequence of ports pointers for node $v$. In order to choose a uniform random permutation of $\{0,1, \ldots, \operatorname{deg}(v)-1\}$ (see [79] Chapter 4), we set $j=\operatorname{deg}(v)$, and call $\operatorname{Random}(j)$ to choose a random number $i$ from $0,1, \ldots, j$. Then, numbers in position $i$ and $j$ 
are interchanged. Afterwards, a random number $i$ from $0, \ldots, j-1$ is selected and the numbers in position $i$ and $j-1$ are interchanged, and so on. This method guarantees that the ports' pointers of $v$, portspoint $(v)$, are chosen randomly among all possible permutations while each of them has equal chance to be chosen.

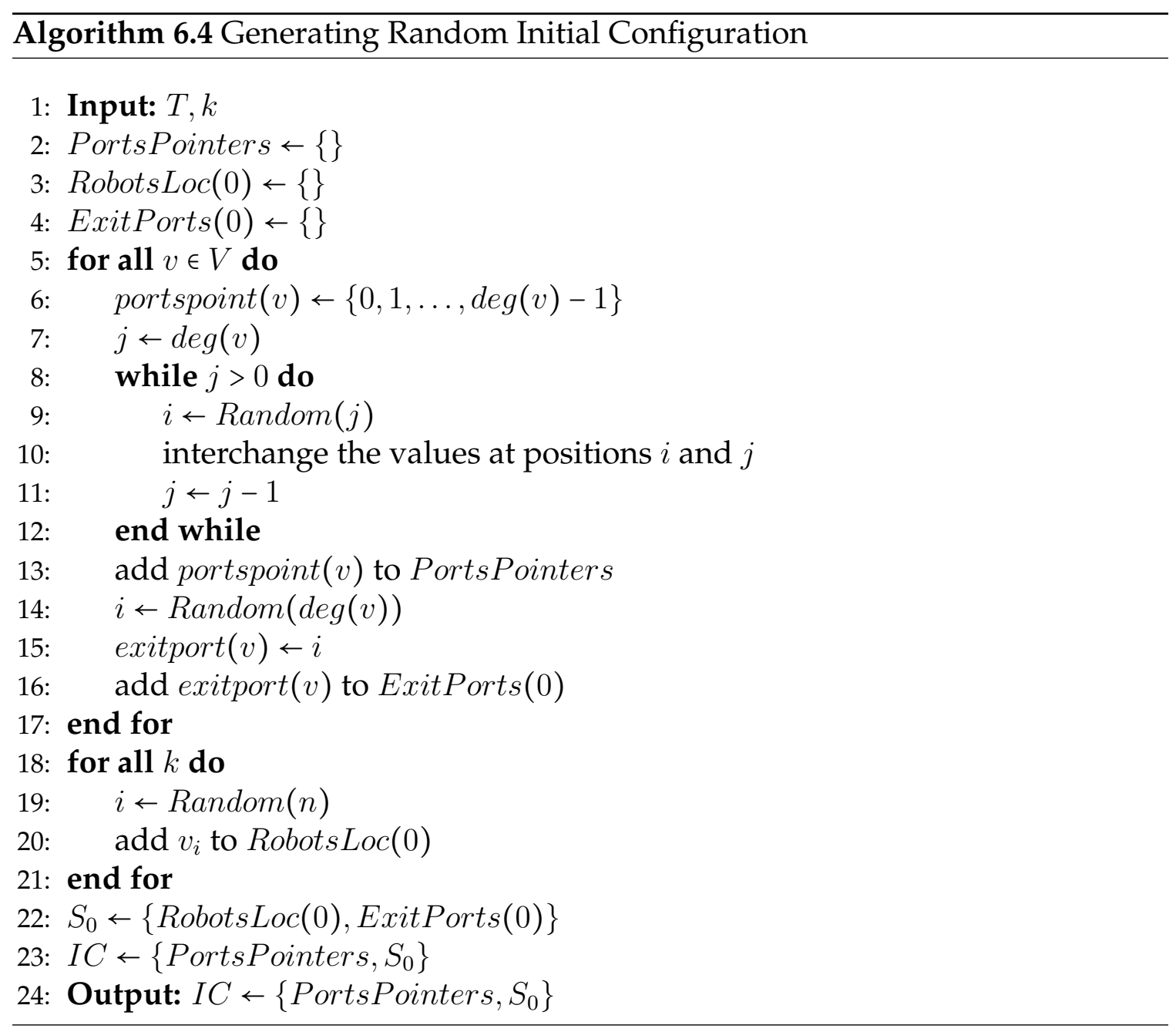

Random Exit Ports. Any port number from $0,1, \ldots, \operatorname{deg}(v)-1$ can be randomly selected as the exit port for node $v$. For this purpose, function $\operatorname{Random}(\operatorname{deg}(v))$ is 
called to generate a random number $i$ from 0 to $\operatorname{deg}(v)-1$. Then, $i$ is assigned as the exit port at $v$, i.e., $\operatorname{exitport}(v)=i$.

Random Robots' Locations. To initialize the system, all $k$ robots should be randomly placed on $k$ nodes of tree $T$ with $n$ nodes where $V=\left\{v_{0}, v_{1}, \ldots, v_{n-1}\right\}$. For this purpose, Algorithm 6.4 calls Random $(n)$ to generate a random number $i$ from 0 to $n-1$, and consider node $v_{i}$ as one of the initial location for the robots. This process will be repeated until $k$ nodes have been selected randomly as the initial locations for $k$ robots.

\subsection{Applying Rotor-Router}

After generating a random tree $T$ and initial configuration $I C=\left\{\right.$ PortsPointers,$\left.S_{0}\right\}$, the system is ready to apply the rotor-router algorithm. For this purpose, Algorithm 6.5 is called to run rotor-router, and compute the cover time $C(T, I C)$, lock-in time $L(T, I C)$, as well as periodicity $P(T, I C)$ for the given $T$ with initial configuration $I C$.

Considering $S_{0}=\{\operatorname{RobotsLoc}(0), \operatorname{ExitPorts}(0)\}$ as the initial state of the system, the robots start moving following the rotor-router mechanism by calling Algorithm 6.6. Algorithm 6.6 demonstrates the robots' movements within a time step $t$. We are assuming at the beginning of time step $t$, the system is at the state of the previous time step, that is $S_{t-1}$. Then, as the robots move within time step $t$, the system state' parameters (robots' locations and exit ports) are updated, and finally $S_{t}$ is generated at the end of time step $t$. For instance, at the beginning of time step 1 , the system state is $S_{0}$. Then, as the robots move within time step 1, the system 


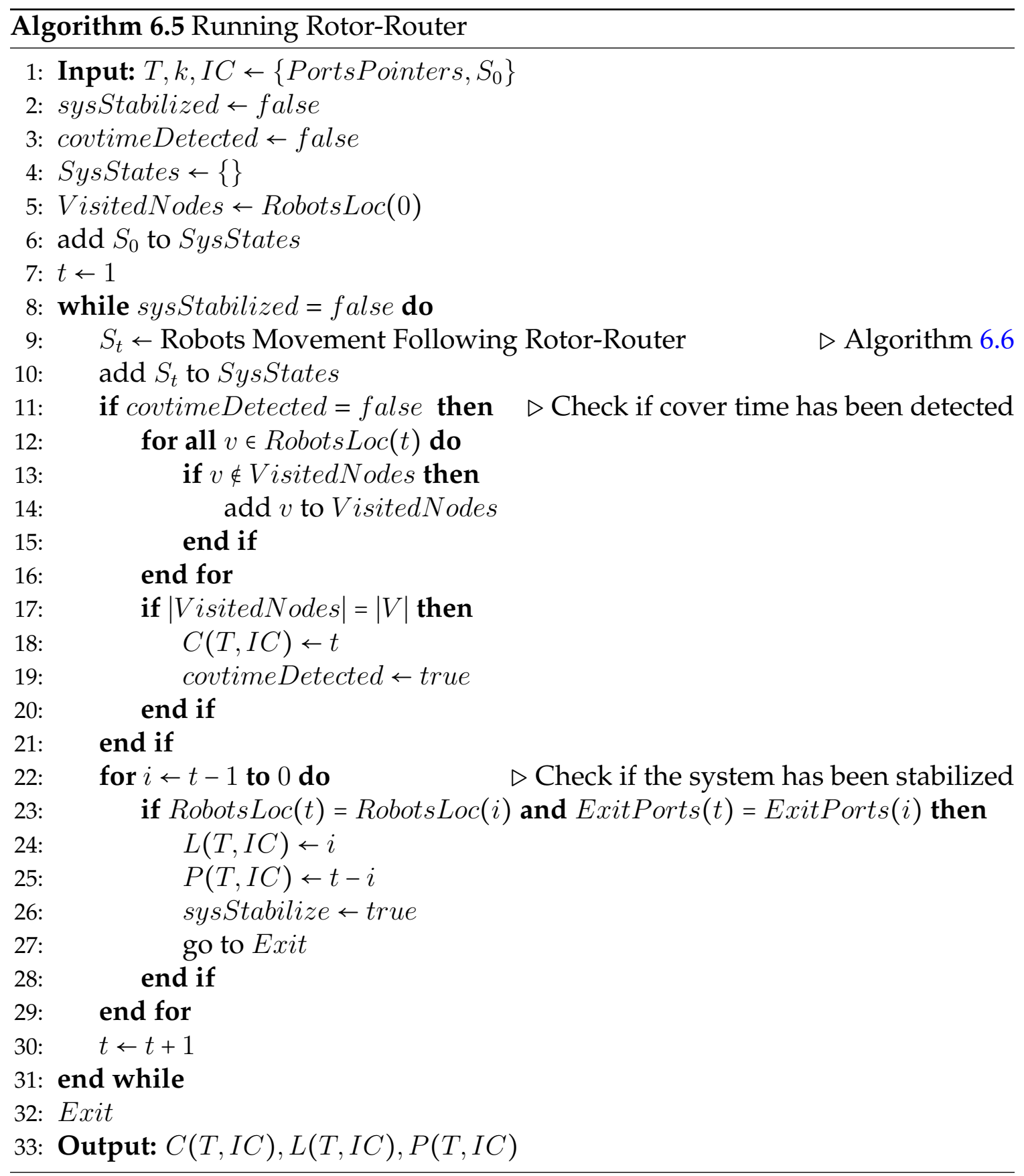


parameters RobotsLoc(0) and ExitPorts(0) are updated, and at the end of time step 1, $S_{1}=\{$ RobotsLoc(1), ExitPorts(1) $\}$ is generated. Then $S_{1}$ is the system state at the beginning of time step 2, and $S_{2}$ is generated at the end of time step 2 , and so on.

Rotor-router is a synchronized algorithm in such a way that all robots enter time step $t$ together, then all of them together enter the next time step $t+1$, and so on. Within time step $t$, each robot considers the edge $e(v, u)$ pointed by the exit post exitport $(v)$, where $v$ is the node where the robot is currently located at. Before leaving $v$, the robot updates exitport $(v)$ based on the rotor-router rules. If more than one robot is located at $v$, access to exitport $(v)$ is mutual exclusion, such that robots access and update exitport $(v)$ one by one. After considering the edge $e(v, u)$ and updating the exit port exitport(v), each robot moves to its new location $u$, which is the other end of $e(v, u)$.

In order to compute the cover time $C(T, I C)$, a list of all visited nodes from the beginning $(t=0)$ to time step $t$ are accumulated in a list denoted by VisitedNodes. At the end of each time step $t$, VisitedNodes is updated since robots have visited some new nodes within time step $t$. Consequently, if VisitedNodes contains all the nodes of $T$, i.e., VisitedNodes $=|V|$, the cover time has been detected at time step $t$, and $C(T, I C)=t$. Algorithm 6.5 uses a boolean variable, denoted by covtimeDetected, to stop looking for the cover time once it is detected.

In order to check stabilization, a list of all systems states denoted by SysStates, from the beginning $(t=0)$ to time step $t$ are reserved, i.e, SysStates $\left\{S_{0}, S_{1}, \ldots, S_{t}\right\}$. To see if the system has been stabilized at time step $t, S_{t}$ is compared with all the previous states at SysStates (from $S_{t-1}$ to $S_{0}$ ) until an equal state is found, or the 
list ends. Two states are equal if and only if the robots' locations, and exit ports of the states are equal. In other words, $S_{t}=S_{t^{\prime}} \Leftrightarrow \operatorname{ExitPorts}(t)=\operatorname{ExitPorts}\left(t^{\prime}\right)$ and $\operatorname{RobotsLoc}(t)=\operatorname{RobotsLoc}\left(t^{\prime}\right)$. Thus, if an equal state with the current state $S_{t}$ is detected such that $S_{t}=S_{t^{\prime}}$, where $0 \leq t^{\prime}<t$, the system has been stabilized at $t^{\prime}$, and the lock-in time and periodicity are detected, i.e., $L(T, I C)=t^{\prime}$ and $P(T, I C)=t-t^{\prime}$.

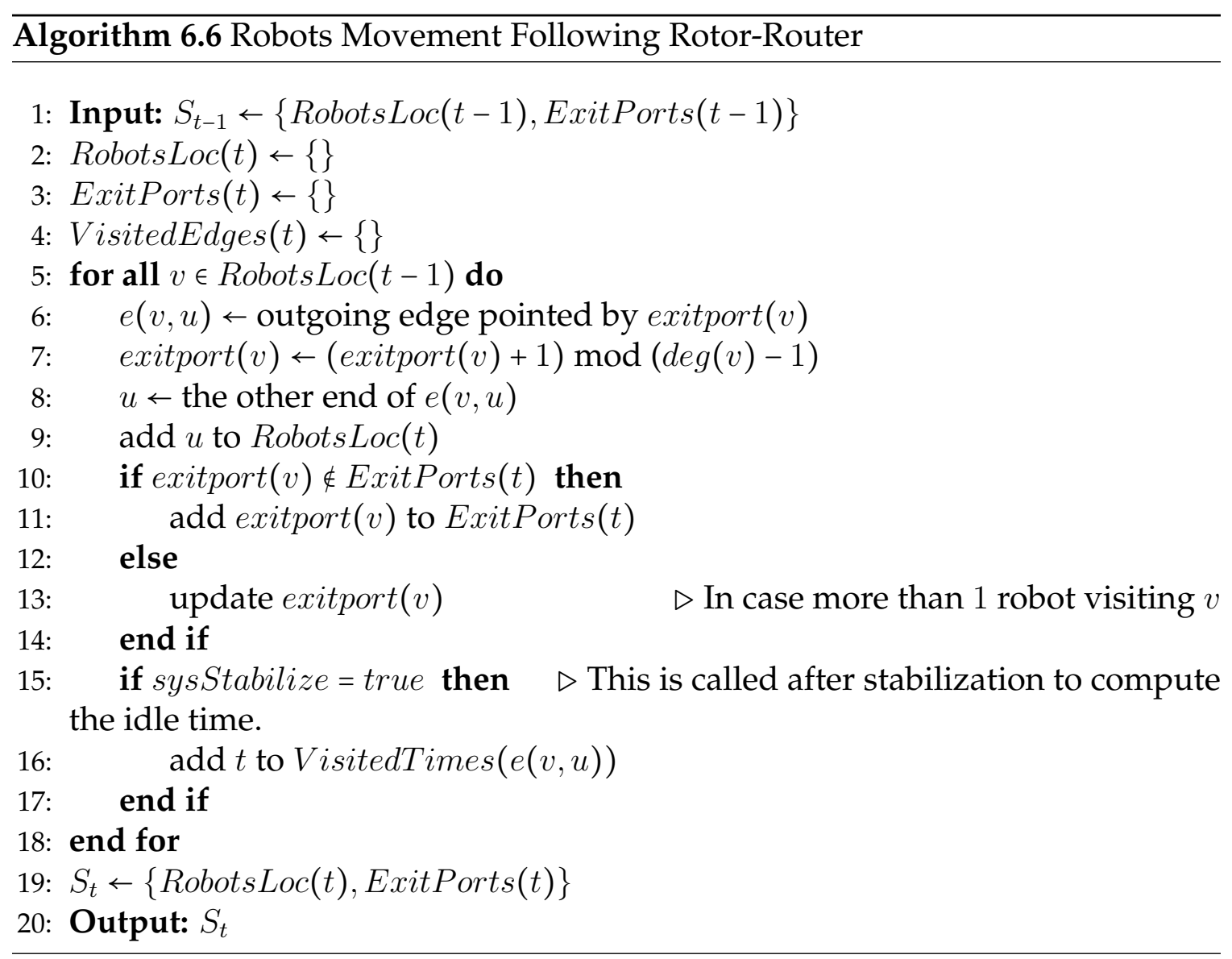




\subsection{Computing Idle Time}

Once the system stabilizes, we are able to compute the idle time since stabilization guarantees that the system states will be repeated periodically in the future, and consequently all the edges will be visited perpetually by the robots. The rotorrouter simulator applies Algorithm 6.7 to compute the idle time $I(T, I C)$ for tree $T$ considering initial configuration $I C$. The idle time of $T$ is in fact the maximum idle time among all its edge's idle times, and an edge idle time is the longest time interval between two consecutive visits to the edge.

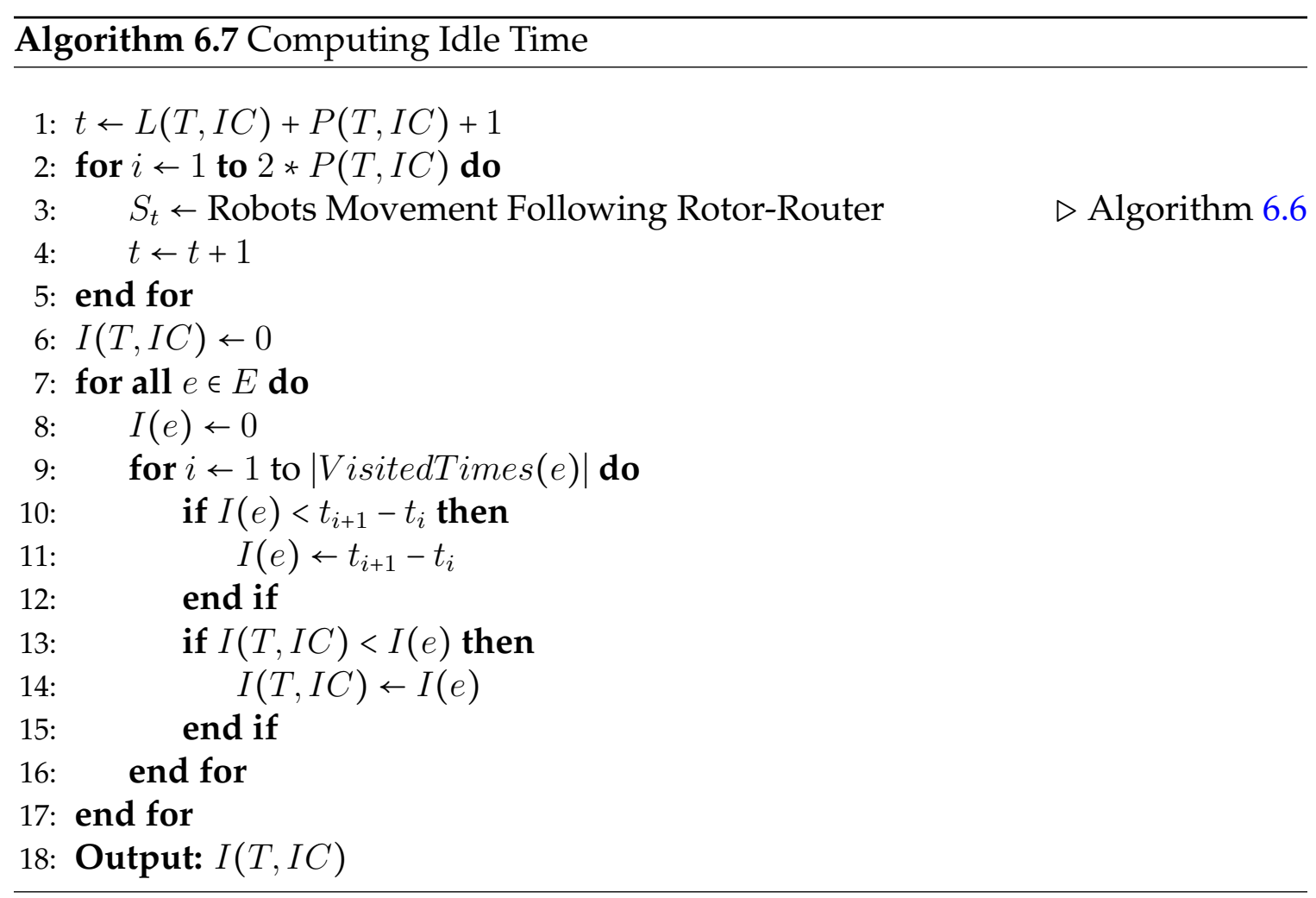

In order to compute the tree idle time $I(T, I C)$, we run rotor-router algorithm after stabilization for two consecutive stable periods (a time interval from time step $t$ to $t+P(T, I C)$ is considered as a stable period) to assure that each edge has been 
visited adequately to compute its idle time. As rotor-router is running, we keep a list of time steps for each edge $e$, denoted by visitedTimes(e), during which $e$ was visited. To compute the idle time of edge $e$, denoted by $I(e)$, we need to subtract every two consecutive time steps in visitedTimes(e) since it achieves the idle time of two consecutive visits of $e$. Therefore, we subtract all successive $t \in$ visitedTimes $(e)$, i.e., $t_{i}-t_{i-1}$ for all $i=1, \ldots, \mid$ visitedTimes $(e) \mid$, and take the maximum one as the edge's idle time $I(e)$. Finally, we take the maximum idle time among all the edges as the idle time of tree $I(T, I C)$.

\subsection{Conclusion and Open Problems}

In this chapter, we discussed the implementation of the rotor-router simulator implemented in Java. In order to study the effect of initial configurations and tree structure on the rotor-router algorithm, we needed to run experiments on the simulator due to the complexity of the algorithm, as well as the extremely large number of cases (initial configurations and tree structure) to be tested. To run experiments, the simulator uses the Aldous-Broder algorithm to generate uniform random trees for a given number of nodes $n$. It also applies appropriate procedures to generate uniform random initial configurations for a given tree $T$ and a set of $k$ robots. For each experiment on $T$ considering the initial configuration $I C$, the cover time $C(T, I C)$, lock-in time $L(T, I C)$, periodicity $P(T, I C)$, and idle time $I(T, I C)$ are computed. The results of each experiment are saved in external files for further analysis. The rotor-router simulator developed in this study can be further extended to incorporate new features. For instance, it will be interesting 
if the simulator detects the sub-cycle decomposition for a given tree $T$ and initial configuration $I C$. 


\section{Chapter 7}

\section{Conclusion and Future Work}

\subsection{Conclusion}

Patrolling is a task that occurs in many common daily activities whenever it is required to monitor a specific region, for example, the perimeter of a piece of land or a building, so as to investigate a feature of interest for the purposes of surveillance. Typically, in such a setting, patrolmen (robots) are assigned to monitor specified regions by moving perpetually at regular intervals through the areas assigned to them. The purpose of patrolling could be looking for any signs of specific problems (for example, responding to service calls, resolving disputes and/or making arrests, reporting crime, and conducting traffic enforcement) which need to be reported. Recently, patrolling problem has been of great interest not only because of its numerous real life applications, but also due to its close connection with known theoretical problems.

From the theoretical perspective, patrolling is tightly connected to very wellknown problems in computer science, such as "coverage", "exploration", and "art gallery" problems. For instance, patrolling may be considered as the continuous 
coverage (see, e.g., $[17,85,86])$, or the ongoing exploration of an unknown environment (see, e.g., [89, 90, 92]). Moreover, there are a wide variety of known problems that could be reformulated as patrolling tasks. For instance, "searching" a graph or polygon by a team of mobile robots, which are looking for a stationary or mobile intruder (see, e.g., $[47,95]$ ), may be considered as the randomized patrolling in an adversarial setting.

Many studies have been conducted to investigate the patrolling problem from theoretical and experimental perspectives. In general, two approaches can be found in the literature: cyclic strategy, where robots move in one direction around the cycle covering the environment, and partition strategy, in which the environment to be patrolled is partitioned into sections patrolled separately by individual robots (or subsets of robots). In all patrolling algorithms, the idleness has been considered as the fundamental measure for evaluating the efficiency of the proposed strategies. The idle time (idleness) is in fact, the maximum time (steps) when a point of the terrain remains unseen by any robot.

Minimizing the idle time is the main goal of all the proposed patrolling algorithms. Considering this common goal, many strategies have been proposed for performing patrolling tasks considering different settings. We classified (in Chapter 2) the existing patrolling strategies based on the environment to be patrolled, and the strategies used to solve the problem. According to our classification, every patrolling algorithm belongs to one of the following four categories: area patrolling, perimeter patrolling, randomized patrolling, and distributed patrolling. In area patrolling, the robots patrol every point of a two dimensional environment, however in perimeter patrolling only the boundary of the environment, represented 
by an open or closed fence (curve), is considered for patrolling. In a totally different adversarial setting, randomized patrolling is performed in the presence of an intruder which is attempting to penetrate the environment. In such a setting, applying deterministic algorithms may not be useful since an intelligent adversary may learn the patrolling strategy, and take advantage of the idle time for penetration. However, if the robots move non-deterministically, the choice of penetration position will be less trivial. In the majority of the proposed patrolling algorithms, a central controller leads the agents to carry out the patrolling task, but in a distributed (decentralized) setting, the robots perform the patrolling task in the absence of a central controller. In such a setting, the robots use different ways for communication, for example, sending messages, leaving tokens, bouncing and colliding, in order to perform patrolling task. Some of the known distributed patrolling strategies are based on swarm or ant-based algorithms, among which rotor-router [91] is one of the most popular ant-based methods.

We approached the patrolling problem considering variant robot models, and patrolling strategies to solve the problem. For the purpose of this study, we considered a continuous and homogeneous domain, in which every point of the domain has the same priority to be patrolled, and robots are able to move along the region using their maximum speed without the need to change their speed. In addition, we assumed a static region, in which there will be no change neither in the state (homogeneous or heterogeneous), nor in the configuration of the region within the execution of patrolling task. As the robot model, we considered a team of $k$ mobile robots, with no memory to remember their environments or the events happening around them. In addition, the robots have no communication tools, however they 
possess the synchronized clocks to perform their patrolling tasks synchronously. In terms of performance criteria, we considered the worse idle time, which is the maximum time when a point of the region is unseen by any robot. Considering these settings, we tackled the patrolling problem as follows: first, we studied patrolling by faulty mobile robots; secondly, we considered patrolling by robots equipped with visibility; and finally, we showed the optimality of an off-line patrolling strategy for trees in the centralized settings, as well as the efficiency of an on-line patrolling strategy in the decentralized (distributed) settings.

First (in Chapter 3), we studied a model of faulty (unreliable) robots with zero visibility, and the same speed. The faulty robots, which are assumed to be unknown, do not report their monitoring tasks to the central controller. We investigated patrolling open fences (segments), and arbitrary graphs by a team of $k$ mobile robots, among which $f$ of them are faulty. The goal of the patrolling task was visiting every point of the environment by a reliable robot as often as possible. A fault-tolerant patrolling strategy was proposed for the segments when $f<\frac{k-2}{2}$. This non-intuitive strategy relies on a decomposition of the set of robots into three groups with different patrolling tours, in a way dependent on $k$ and $f$. It was proved that the proposed algorithm is optimal when $f$ is odd, and near optimal when $f$ is even. Then, it was demonstrated that the cyclic strategy is optimal for Eulerian graphs for any ration of $f$ and $k$. Finally, it was proved that finding an optimal patrolling strategy for some arbitrary graphs is NP-hard. For this purpose, it was shown that if the idle time could be computed optimally, then we could solve "3-edge-coloring" of cubic graphs (a well known NP-hard problem [49]).

Secondly (in Chapter 4), we studied a model of reliable robots equipped with 
visibility, in which every robot $a_{i}$ has a range of visibility $r_{i}$, representing the distance from its current position, at which the robot can see in each direction along the curve. We considered patrolling the open and closed fences, as well as general graphs with the goal of minimizing the idle time. When all robots have the same speed but different visibility ranges, we showed that a version of the cyclic strategy is optimal for patrolling the closed fences. Likewise, it was demonstrated that the partition strategy achieves the optimal idle time for the open fences. Afterward, we briefly discussed the case of fence (either open or closed) patrolling by robots with distinct speeds and visibility ranges, to show that fence patrolling by robots with distinct visibility ranges is entirely different than the case of robots with zero visibility. Nevertheless, we proposed an optimal algorithm for patrolling the open fences by two robots with distinct speeds and visibility ranges. For the case when the environment is a general graph, we showed that the patrolling problem for robots with the same speed and distinct visibility ranges is NP-hard. This contrasts sharply with the case when robots have the same speed and zero visibility, which has been known to have a polynomial-time solution [35].

Although patrolling by a team of reliable robots with the same speeds and zero visibility may look straightforward, no optimal algorithm for patrolling general graphs has been proved yet. Therefore, in order to get closer to a solution for this problem, we took one step further and studied patrolling trees by a team of $k$ homogeneous mobile robots (i.e., reliable robots with the same speeds and zero visibility) in Chapter 5. We first studied patrolling a geometric tree $T$, where agents can change direction and speed anywhere on the vertices or interiors of edges. We 
proposed an optimal off-line (i.e., the environment to be patrolled is known) patrolling strategy, in which $k$ robots traverse the geometric tree at their maximum speed along an Eulerian cycle while they are equidistant. Then, we extended this optimal algorithm to the graph tree $T$, where the patrolling robots are allowed to change direction only at vertices of the tree. Afterwards, we utilized these results to study patrolling trees in a distributed (decentralized) setting by a team of $k$ mobile robots following the rotor-router mechanism. It has been theoretically proved that rotor-router achieves the idle time of $c . I(T)$ for trees, where $c=6$, and $I(T)$ is the optimal idle time of the centralized algorithm (proposed for tree T) [63]. However, we experimentally showed that the resulting rotor-router has a competitive ratio at least $4 / 3$. In other words, according to our experimental results, $c=4 / 3$ may be the least possible value.

Rotor-router algorithm was originally proposed as a deterministic alternative to "random walk" for the exploration of unknown environments. Besides being distributed, the rotor-router is also an on-line algorithm (i.e., the environment to be patrolled is unknown), which makes it a practical tool for patrolling an unknown environment as well. The basic idea of rotor-router is to set locally shared memories at the nodes of the tree $T$. Subsequently, the robots will be updating these shared memories as they visit the vertices of the tree. In fact, the periodic behavior (see $[21,96])$ of rotor-router makes it a practical tool for distributed patrolling. We simulated the rotor-router algorithm (called rotor-router simulator (Chapter 6)), in order to study the idle time of this distributed strategy for trees.

It has been shown (see, e.g., [12]) that in addition to the given number of nodes and robots ( $n$ and $k$, respectively), the following non-quantitative factors may also 
affect the rotor-router performance (and consequently the idle time achieved by this strategy): the initial configuration of the (rotor-router) system, which is defined by the port's pointers, initial exit ports, and initial locations of robots, as well as tree structures. Beside the complex behavior of parallel rotor-routers, there are also numerous number of initial configurations (for given $n$ and $k$ ), and tree structures (for given $n$ ) to consider. Therefore, we ran experiments on the rotor-router simulator for the randomly generated trees and initial configurations to observe the effects of these non-quantitative factors on the idle time.

Our experimental results showed that the distributed patrolling strategy (following rotor-router) achieves nearly optimal idle time for trees. In fact, rotor-router achieved the optimal idle time for the most number of tree structures and initial configurations over 250, 000 experiments. However, there were a few cases (tree structure and initial configurations) where the achieved idle time was not optimal. In these cases, the idle time was nearly $\frac{4}{3} I(T)$, where $I(T)$ is the optimal idle time of the centralized algorithm for trees. We may conclude that rotor-router is an on-line distributed patrolling algorithm which is certainly efficient for trees.

Although there have been many studies toward patrolling problem, there are still many open problems considering variants environments and robots models. In the following section, we list some of the questions raised during conducting this study. 


\subsection{Future Work}

There are many directions that can be pursued to research patrolling problems. These include, considering various robot models, for instance, faulty robots, robots equipped with visibility, or robots with different speeds, patrolling strategies used to solve the problem, for example, centralized vs. decentralized algorithms, as well as the region to be patrolled, for instance, homogeneous vs. heterogeneous regions, or known vs. unknown regions.

Patrolling in an unreliable setting, in which some of the robots may be faulty (i.e., they do not report to the central controller, and are unknown to the rest of team) is very realistic in real life applications of patrolling tasks. We proposed an optimal patrolling strategy for the segments, considering the faulty robots model,

and assuming that the maximum faulty robots ratio is slightly smaller than half the total number of all robots. One open question is to give optimal patrolling strategies for segments when the faulty robot ratio is high. There are many open questions concerning different models of faulty robots. For instance, robot failures may be dynamic, i.e., number of faulty robots may change, or failures may happen with a given probability.

Patrolling by robots with different speeds may be more applicable in real life scenarios since we may have a team of robots with different characteristics and capabilities. In these circumstances, proposing an efficient strategy requires using (or even not using) the robots and their speeds appropriately. The research toward this problem has progressed slowly since the problem is intrinsically challenging, even for regions as simple as line segments. The problems of finding optimal algorithms (if there are any) for any configuration of speed settings for $k>3$ robots patrolling 
an open or closed fence are yet to be solved. The problem for general graphs may be even more challenging. Although this problem is very difficult from a theoretical perspective, using simulation models may accelerate the progress toward finding efficient patrolling strategies for any number of robots with different speeds.

In the majority of patrolling problems, the robots model is assumed to be homogeneous (reliable robots with same speed and zero visibility). In some studies, on the other hand, the robots model has slightly changed, for instance, a team of $k$ reliable robots with distinct speeds and zero visibility. Nevertheless, more sophisticated models, where the robots have different speeds and visibility while some of them may be faulty, have not been studied yet. Moreover, most studies assume an ideal robots model, in that the robots have unlimited battery power, or never break down. However, all these constraints should be taken into account in the real life applications. In addition to these constrains, there may be an adversary attempting to penetrate the environment. The existence of an adversary may entirely change the problem settings.

The presence of an intelligent central controller in order to coordinate the robots, and assign them their patrolling tasks, may simplify the patrolling problem. However, in some circumstances having a central controller may be very expensive if not impossible. In such a setting, it is crucial that robots are enable to perform their patrolling tasks autonomously and scatterly. In the current study, we investigated the distributed patrolling following rotor-router strategies for trees. However, the problem of distributed patrolling for general graphs is still open. It would be also interesting to investigate the rotor-router mechanism for heterogeneous robots model, for instance, robots with different speeds and visibility ranges, or even 
faulty robots. Moreover, as we experimentally showed, the rotor-router mechanism achieves the nearly optimal idle time for trees (i.e., it achieves the optimal idle time for most tree structures, but not all of them). There may be some other distributed mechanisms that guarantee the optimal idle time for all tree structures.

In addition to the robots model, considering various environmental settings such as heterogeneous environments, dynamically changing environments, or unknown environments, may raise many interesting open problems. For instance, patrolling an environment where the difficulty of passing through its terrains vary from one terrain to another, for example, driving in muddy tracks vs. driving on paved roads. Another example is an environment, where different parts (regions) of it have different priorities to be patrolled. For instance, some areas of an environment (e.g., an international airport) may be required to be visited more frequently. In addition to the heterogeneous environmental settings, patrolling in dynamically changing environments could be very challenging. One example is patrolling in an environment with moving obstacles, for instance, patrolling in a shopping center where people are the moving obstacles. Finally, on-line patrolling when the environment to be patrolled is unknown could pose many interesting problems. Performing on-line patrolling is critical when providing a map of the environment is expensive (in terms of time or cost), or even impossible. For instance, providing a quick map of the interior parts of a damaged building after the earthquake may not be feasible, and thus on-line patrolling would become necessary. 
Appendices 


\section{Appendix A}

\section{Rotor-Router Experimental Results for a Random Tree}

The experimental results illustrated in Tables A.1, A.2, A.3, and A.4 are obtained by running experiments on the rotor-router simulator for a random tree with 50 nodes, and 100 random initial configurations for each set of $k=1,2, \ldots, 50$ robots, i.e., 5, 000 experiments were run in total. 


\begin{tabular}{|c|c|c|c|c|c|c|}
\hline $\mathrm{K}$ & $\begin{array}{l}\text { Minimum } \\
\text { CoverTime }\end{array}$ & $\begin{array}{l}\text { Maximum } \\
\text { CoverTime }\end{array}$ & $\begin{array}{l}\text { Average } \\
\text { CoverTime }\end{array}$ & $\begin{array}{l}\text { Standard } \\
\text { Deviation }\end{array}$ & \multicolumn{2}{|c|}{$\begin{array}{c}\text { 95\% Confidence } \\
\text { Interval }\end{array}$} \\
\hline 1 & 213 & 593 & 404.63 & 87.72 & 387.44 &, 421.82 \\
\hline 2 & 83 & 337 & 190.9 & 60.2 & 179.10 & ,202.70 \\
\hline 3 & 56 & 265 & 127.96 & 42.12 & 119.70 & ,136.22 \\
\hline 4 & 47 & 204 & 97.38 & 40.02 & 89.54 & ,105.22 \\
\hline 5 & 35 & 190 & 74.09 & 27.71 & 68.66 & ,79.52 \\
\hline 6 & 32 & 163 & 63.06 & 24.79 & 58.20 & ,67.92 \\
\hline 7 & 22 & 131 & 54.07 & 20.06 & 50.14 &, 58.00 \\
\hline 8 & 21 & 97 & 44.65 & 14.44 & 41.82 & ,47.48 \\
\hline 9 & 22 & 109 & 40.56 & 16.04 & 37.42 & ,43.70 \\
\hline 10 & 17 & 84 & 36.47 & 14.05 & 33.72 & ,39.22 \\
\hline 11 & 16 & 62 & 31.39 & 9.61 & 29.51 & ,33.27 \\
\hline 12 & 13 & 63 & 31.05 & 10.75 & 28.94 & ,33.16 \\
\hline 13 & 13 & 68 & 26.21 & 9.49 & 24.35 & ,28.07 \\
\hline 14 & 10 & 54 & 24.01 & 8.56 & 22.33 & ,25.69 \\
\hline 15 & 11 & 51 & 23.49 & 8.3 & 21.86 & ,25.12 \\
\hline 16 & 10 & 56 & 21.36 & 7.88 & 19.82 & ,22.90 \\
\hline 17 & 9 & 45 & 20.3 & 6.77 & 18.97 & ,21.63 \\
\hline 18 & 9 & 45 & 19.37 & 6.03 & 18.19 & ,20.55 \\
\hline 19 & 6 & 38 & 17.6 & 6.17 & 16.39 &, 18.81 \\
\hline 20 & 8 & 44 & 16.69 & 6.06 & 15.50 & ,17.88 \\
\hline 21 & 8 & 37 & 16.78 & 6.5 & 15.51 &, 18.05 \\
\hline 22 & 5 & 45 & 14.14 & 5.55 & 13.05 & ,15.23 \\
\hline 23 & 6 & 32 & 14.52 & 5.13 & 13.51 &, 15.53 \\
\hline 24 & 6 & 44 & 14.06 & 4.81 & 13.12 & 15.00, \\
\hline 25 & 6 & 33 & 12.92 & 4.68 & 12.00 & ,13.84 \\
\hline 26 & 5 & 26 & 12.28 & 4.2 & 11.46 & 13.10 \\
\hline 27 & 5 & 28 & 11.49 & 4.37 & 10.63 &, 12.35 \\
\hline 28 & 4 & 24 & 10.9 & 3.58 & 10.20 & ,11.60 \\
\hline 29 & 4 & 24 & 10.76 & 3.81 & 10.01 & ,11.51 \\
\hline 30 & 5 & 26 & 10.32 & 3.9 & 9.56 & ,11.08 \\
\hline 31 & 4 & 26 & 9.87 & 3.96 & 9.09 & ,10.65 \\
\hline 32 & 4 & 21 & 9.04 & 3.2 & 8.41 &, 9.67 \\
\hline 33 & 5 & 20 & 9.28 & 3.15 & 8.66 & ,9.90 \\
\hline 34 & 4 & 21 & 8.85 & 3.14 & 8.23 & 9.47 \\
\hline 35 & 4 & 18 & 8.87 & 2.97 & 8.29 & ,9.45 \\
\hline 36 & 4 & 17 & 7.52 & 2.41 & 7.05 & 7.99 \\
\hline 37 & 3 & 16 & 7.78 & 2.7 & 7.25 & ,8.31 \\
\hline 38 & 3 & 18 & 7.62 & 3.02 & 7.03 & ,8.21 \\
\hline 39 & 3 & 16 & 7.41 & 2.95 & 6.83 & 7.99 \\
\hline 40 & 3 & 20 & 6.8 & 2.89 & 6.23 & ,7.37 \\
\hline 41 & 3 & 16 & 7.03 & 2.77 & 6.49 & ,7.57 \\
\hline 42 & 3 & 28 & 6.82 & 3.34 & 6.17 & ,7.47 \\
\hline 43 & 3 & 16 & 6.77 & 2.64 & 6.25 & ,7.29 \\
\hline 44 & 3 & 12 & 6.79 & 2.16 & 6.37 & ,7.21 \\
\hline 45 & 3 & 16 & 6.18 & 2.26 & 5.74 & ,6.62 \\
\hline 46 & 3 & 16 & 5.83 & 2.36 & 5.37 & 6.29 \\
\hline 47 & 2 & 12 & 5.75 & 2.08 & 5.34 & ,6.16 \\
\hline 48 & 3 & 11 & 5.66 & 1.94 & 5.28 & 6.04 \\
\hline 49 & 3 & 12 & 5.69 & 2.1 & 5.28 & ,6.10 \\
\hline 50 & 3 & 16 & 5.77 & 2.46 & 5.29 & ,6.25 \\
\hline
\end{tabular}

TABLE A.1: Cover times obtained by applying rotor-router on a random tree with 50 nodes, and 100 distinctive initial configurations for each set of $k=1,2, \ldots, 50$ robots. 


\begin{tabular}{|c|c|c|c|c|c|c|}
\hline $\mathrm{K}$ & $\begin{array}{c}\text { Minimum } \\
\text { Lock-inTime }\end{array}$ & $\begin{array}{l}\text { Maximum } \\
\text { Lock-inTime }\end{array}$ & $\begin{array}{c}\text { Average } \\
\text { Lock-inTime }\end{array}$ & $\begin{array}{l}\text { Standard } \\
\text { Deviation }\end{array}$ & \multicolumn{2}{|c|}{$\begin{array}{c}95 \% \text { Confidence } \\
\text { Interval }\end{array}$} \\
\hline 1 & 215 & 595 & 405.89 & 87.62 & 388.72 & ,423.06 \\
\hline 2 & 132 & 1643 & 799.34 & 472.93 & 706.65 & ,892.03 \\
\hline 3 & 202 & 1539 & 652.72 & 304.87 & 592.96 & ,712.48 \\
\hline 4 & 161 & 1692 & 817.64 & 425.81 & 734.18 & ,901.10 \\
\hline 5 & 203 & 1276 & 735.49 & 253.1 & 685.88 &, 785.10 \\
\hline 6 & 162 & 1154 & 610.7 & 222.98 & 567.00 & ,654.40 \\
\hline 7 & 56 & 665 & 335.98 & 166.19 & 303.41 & ,368.55 \\
\hline 8 & 190 & 1620 & 800.54 & 331.82 & 735.50 & ,865.58 \\
\hline 9 & 261 & 1483 & 825.45 & 317.82 & 763.16 & ,887.74 \\
\hline 10 & 242 & 1339 & 848.94 & 243.75 & 801.16 & ,896.72 \\
\hline 11 & 176 & 1260 & 718.26 & 244.19 & 670.40 & ,766.12 \\
\hline 12 & 161 & 1188 & 732.27 & 223.69 & 688.43 &, 776.11 \\
\hline 13 & 224 & 1706 & 870.92 & 322.7 & 807.67 & ,934.17 \\
\hline 14 & 106 & 649 & 390.7 & 136.78 & 363.89 &, 417.51 \\
\hline 15 & 296 & 1666 & 815.51 & 330.75 & 750.68 & ,880.34 \\
\hline 16 & 280 & 1154 & 758.38 & 215.78 & 716.09 & 800.67 \\
\hline 17 & 247 & 1526 & 988.75 & 315.15 & 926.98 & ,1050.52 \\
\hline 18 & 265 & 1406 & 910.33 & 274.43 & 856.54 &, 964.12 \\
\hline 19 & 324 & 1367 & 923.39 & 222.05 & 879.87 & ,966.91 \\
\hline 20 & 300 & 1410 & 876.31 & 259.93 & 825.36 & ,927.26 \\
\hline 21 & 81 & 680 & 397.59 & 151.26 & 367.94 & ,427.24 \\
\hline 22 & 335 & 1309 & 913.36 & 194.82 & 875.18 & ,951.54 \\
\hline 23 & 167 & 1482 & 849.41 & 308.91 & 788.86 & ,909.96 \\
\hline 24 & 262 & 1050 & 789.23 & 178.68 & 754.21 & ,824.25 \\
\hline 25 & 189 & 1034 & 768.03 & 216.69 & 725.56 & 810.50 \\
\hline 26 & 213 & 1371 & 822.27 & 287.9 & 765.84 & ,878.70 \\
\hline 27 & 359 & 1387 & 934.26 & 191.5 & 896.73 & ,971.79 \\
\hline 28 & 107 & 681 & 421.81 & 145.21 & 393.35 & ,450.27 \\
\hline 29 & 202 & 1430 & 866.33 & 279.74 & 811.50 & ,921.16 \\
\hline 30 & 255 & 1388 & 954.3 & 224.5 & 910.30 & ,998.30 \\
\hline 31 & 282 & 1621 & 973.14 & 286.37 & 917.01 & ,1029.27 \\
\hline 32 & 237 & 1512 & 1008.72 & 284.87 & 952.89 &, 1064.55 \\
\hline 33 & 258 & 1194 & 834.89 & 206.88 & 794.34 &, 875.44 \\
\hline 34 & 196 & 1717 & 911.85 & 407.29 & 832.02 & ,991.68 \\
\hline 35 & 103 & 738 & 459.76 & 158.35 & 428.72 & ,490.80 \\
\hline 36 & 326 & 1893 & 946.47 & 333.69 & 881.07 & ,1011.87 \\
\hline 37 & 201 & 1349 & 809.07 & 252.55 & 759.57 & ,858.57 \\
\hline 38 & 275 & 1353 & 781.38 & 226.73 & 736.94 & ,825.82 \\
\hline 39 & 223 & 1438 & 893.94 & 240.55 & 846.79 & ,941.09 \\
\hline 40 & 281 & 1560 & 925.48 & 317.68 & 863.22 & ,987.74 \\
\hline 41 & 321 & 1705 & 937.75 & 302.55 & 878.45 & ,997.05 \\
\hline 42 & 121 & 848 & 462.62 & 166.59 & 429.97 & ,495.27 \\
\hline 43 & 203 & 1340 & 715.21 & 248.87 & 666.43 & ,763.99 \\
\hline 44 & 333 & 1406 & 824.59 & 241.56 & 777.24 & ,871.94 \\
\hline 45 & 201 & 1798 & 1024.38 & 357.42 & 954.33 & ,1094.43 \\
\hline 46 & 310 & 1826 & 832.53 & 340.06 & 765.88 & ,899.18 \\
\hline 47 & 329 & 2028 & 1170.31 & 463.32 & 1079.50 & ,1261.12 \\
\hline 48 & 246 & 1118 & 749.61 & 194.73 & 711.44 & ,787.78 \\
\hline 49 & 17 & 416 & 192.84 & 110.19 & 171.24 & ,214.44 \\
\hline 50 & 277 & 1091 & 757.03 & 225.91 & 712.75 & ,801.31 \\
\hline
\end{tabular}

TABLE A.2: Lock-in times obtained by applying rotor-router on a random tree with 50 nodes, and 100 distinctive initial configurations for each set of $k=1,2, \ldots, 50$ robots. 


\begin{tabular}{|c|c|c|c|c|c|c|}
\hline$K$ & $\begin{array}{l}\text { Minimum } \\
\text { Periodicity }\end{array}$ & $\begin{array}{l}\text { Maximum } \\
\text { Periodicity }\end{array}$ & $\begin{array}{c}\text { Average } \\
\text { Periodicity }\end{array}$ & $\begin{array}{l}\text { Standard } \\
\text { Deviation }\end{array}$ & $\begin{array}{r}95 \% \mathrm{C} \\
\text { In }\end{array}$ & $\begin{array}{l}\text { idence } \\
\text { al }\end{array}$ \\
\hline 1 & 98 & 98 & 98 & 0 & 98.00 & ,98.00 \\
\hline 2 & 49 & 98 & 75.46 & 24.54 & 70.65 & ,80.27 \\
\hline 3 & 98 & 98 & 98 & 0 & 98.00 & ,98.00 \\
\hline 4 & 49 & 98 & 78.89 & 24.02 & 74.18 & 83.60 \\
\hline 5 & 98 & 98 & 98 & 0 & 98.00 & ,98.00 \\
\hline 6 & 49 & 98 & 85.26 & 21.6 & 81.03 & 89.49 \\
\hline 7 & 14 & 28 & 20.44 & 7.01 & 19.07 & 21.81 \\
\hline 8 & 49 & 98 & 83.79 & 22.35 & 79.41 & ,88.17 \\
\hline 9 & 98 & 98 & 98 & 0 & 98.00 & ,98.00 \\
\hline 10 & 49 & 98 & 87.71 & 20.06 & 83.78 & ,91.64 \\
\hline 11 & 98 & 98 & 98 & 0 & 98.00 & ,98.00 \\
\hline 12 & 49 & 98 & 82.32 & 22.97 & 77.82 & ,86.82 \\
\hline 13 & 98 & 98 & 98 & 0 & 98.00 & ,98.00 \\
\hline 14 & 7 & 28 & 19.6 & 8.26 & 17.98 & 21.22 \\
\hline 15 & 98 & 98 & 98 & 0 & 98.00 & ,98.00 \\
\hline 16 & 49 & 98 & 86.24 & 21.03 & 82.12 & ,90.36 \\
\hline 17 & 98 & 98 & 98 & 0 & 98.00 & ,98.00 \\
\hline 18 & 49 & 98 & 89.18 & 18.92 & 85.47 & ,92.89 \\
\hline 19 & 98 & 98 & 98 & 0 & 98.00 & ,98.00 \\
\hline 20 & 49 & 98 & 86.73 & 20.72 & 82.67 & ,90.79 \\
\hline 21 & 14 & 28 & 19.46 & 6.86 & 18.11 & 20.81 \\
\hline 22 & 49 & 98 & 90.65 & 17.58 & 87.20 &, 94.10 \\
\hline 23 & 98 & 98 & 98 & 0 & 98.00 & ,98.00 \\
\hline 24 & 49 & 98 & 87.71 & 20.06 & 83.78 & ,91.64 \\
\hline 25 & 98 & 98 & 98 & 0 & 98.00 & ,98.00 \\
\hline 26 & 49 & 98 & 90.65 & 17.58 & 87.20 & ,94.10 \\
\hline 27 & 98 & 98 & 98 & 0 & 98.00 & ,98.00 \\
\hline 28 & 7 & 28 & 19.6 & 8.08 & 18.02 & 21.18 \\
\hline 29 & 98 & 98 & 98 & 0 & 98.00 & ,98.00 \\
\hline 30 & 49 & 98 & 90.16 & 18.05 & 86.62 & ,93.70 \\
\hline 31 & 98 & 98 & 98 & 0 & 98.00 & ,98.00 \\
\hline 32 & 49 & 98 & 92.61 & 15.41 & 89.59 & 95.63 \\
\hline 33 & 98 & 98 & 98 & 0 & 98.00 & ,98.00 \\
\hline 34 & 49 & 98 & 91.14 & 17.09 & 87.79 & ,94.49 \\
\hline 35 & 14 & 28 & 19.88 & 6.94 & 18.52 & 21.24 \\
\hline 36 & 49 & 98 & 93.59 & 14.09 & 90.83 & ,96.35 \\
\hline 37 & 98 & 98 & 98 & 0 & 98.00 & ,98.00 \\
\hline 38 & 49 & 98 & 92.61 & 15.41 & 89.59 & ,95.63 \\
\hline 39 & 98 & 98 & 98 & 0 & 98.00 & 98.00 \\
\hline 40 & 49 & 98 & 86.73 & 20.72 & 82.67 & 90.79 \\
\hline 41 & 98 & 98 & 98 & 0 & 98.00 & 98.00 \\
\hline 42 & 7 & 28 & 19.46 & 7.87 & 17.92 & 21.00 \\
\hline 43 & 98 & 98 & 98 & 0 & 98.00 & 98.00 \\
\hline 44 & 49 & 98 & 94.57 & 12.57 & 92.11 & ,97.03 \\
\hline 45 & 98 & 98 & 98 & 0 & 98.00 & 98.00 \\
\hline 46 & 49 & 98 & 90.16 & 18.05 & 86.62 & ,93.70 \\
\hline 47 & 98 & 98 & 98 & 0 & 98.00 & ,98.00 \\
\hline 48 & 49 & 98 & 95.06 & 11.7 & 92.77 & ,97.35 \\
\hline 49 & 2 & 2 & 2 & 0 & 2.00 & ,2.00 \\
\hline 50 & 49 & 98 & 91.63 & 16.56 & 88.38 & ,94.88 \\
\hline
\end{tabular}

TABLE A.3: Periodicities obtained by applying rotor-router on a random tree with 50 nodes, and 100 distinctive initial configurations for each set of $k=1,2, \ldots, 50$ robots. 


\begin{tabular}{|c|c|c|c|c|c|c|}
\hline K & $\begin{array}{l}\text { Minimum } \\
\text { IdleTime }\end{array}$ & $\begin{array}{l}\text { Maximum } \\
\text { IdleTime }\end{array}$ & $\begin{array}{l}\text { Average } \\
\text { IdleTime }\end{array}$ & $\begin{array}{l}\text { Standard } \\
\text { Deviation }\end{array}$ & \multicolumn{2}{|c|}{$\begin{array}{c}\text { 95\% Confidence } \\
\text { Interval }\end{array}$} \\
\hline 1 & 97 & 97 & 97 & 0 & 97.00 &, 97.00 \\
\hline 2 & 49 & 49 & 49 & 0 & 49.00 & ,49.00 \\
\hline 3 & 32 & 33 & 32.29 & 0.46 & 32.20 & ,32.38 \\
\hline 4 & 24 & 25 & 24.47 & 0.5 & 24.37 & ,24.57 \\
\hline 5 & 19 & 20 & 19.65 & 0.48 & 19.56 &, 19.74 \\
\hline 6 & 16 & 17 & 16.38 & 0.49 & 16.28 & ,16.48 \\
\hline 7 & 13 & 15 & 13.47 & 0.52 & 13.37 & ,13.57 \\
\hline 8 & 12 & 13 & 12.29 & 0.46 & 12.20 &, 12.38 \\
\hline 9 & 11 & 11 & 11 & 0 & 11.00 & ,11.00 \\
\hline 10 & 9 & 10 & 9.95 & 0.22 & 9.91 & 9.99 \\
\hline 11 & 9 & 9 & 9 & 0 & 9.00 & ,9.00 \\
\hline 12 & 8 & 9 & 8.66 & 0.48 & 8.57 & ,8.75 \\
\hline 13 & 7 & 8 & 7.9 & 0.3 & 7.84 & ,7.96 \\
\hline 14 & 7 & 7 & 7 & 0 & 7.00 &, 7.00 \\
\hline 15 & 7 & 7 & 7 & 0 & 7.00 &, 7.00 \\
\hline 16 & 6 & 7 & 6.24 & 0.43 & 6.16 & ,6.32 \\
\hline 17 & 5 & 6 & 5.99 & 0.1 & 5.97 & 6.01 \\
\hline 18 & 5 & 6 & 5.88 & 0.33 & 5.82 &, 5.94 \\
\hline 19 & 5 & 5 & 5 & 0 & 5.00 &, 5.00 \\
\hline 20 & 5 & 5 & 5 & 0 & 5.00 &, 5.00 \\
\hline 21 & 5 & 5 & 5 & 0 & 5.00 &, 5.00 \\
\hline 22 & 5 & 5 & 5 & 0 & 5.00 &, 5.00 \\
\hline 23 & 4 & 5 & 4.21 & 0.41 & 4.13 & ,4.29 \\
\hline 24 & 4 & 5 & 4.79 & 0.41 & 4.71 & ,4.87 \\
\hline 25 & 4 & 4 & 4 & 0 & 4.00 & ,4.00 \\
\hline 26 & 4 & 4 & 4 & 0 & 4.00 & ,4.00 \\
\hline 27 & 4 & 4 & 4 & 0 & 4.00 & ,4.00 \\
\hline 28 & 3 & 4 & 3.98 & 0.14 & 3.95 & ,4.01 \\
\hline 29 & 3 & 4 & 3.91 & 0.29 & 3.85 & ,3.97 \\
\hline 30 & 3 & 4 & 3.71 & 0.46 & 3.62 & ,3.80 \\
\hline 31 & 3 & 4 & 3.49 & 0.5 & 3.39 & ,3.59 \\
\hline 32 & 3 & 4 & 3.11 & 0.31 & 3.05 & ,3.17 \\
\hline 33 & 3 & 3 & 3 & 0 & 3.00 & ,3.00 \\
\hline 34 & 3 & 3 & 3 & 0 & 3.00 & ,3.00 \\
\hline 35 & 3 & 3 & 3 & 0 & 3.00 & ,3.00 \\
\hline 36 & 3 & 3 & 3 & 0 & 3.00 & ,3.00 \\
\hline 37 & 3 & 3 & 3 & 0 & 3.00 & ,3.00 \\
\hline 38 & 3 & 3 & 3 & 0 & 3.00 & ,3.00 \\
\hline 39 & 3 & 3 & 3 & 0 & 3.00 & ,3.00 \\
\hline 40 & 3 & 3 & 3 & 0 & 3.00 & ,3.00 \\
\hline 41 & 3 & 3 & 3 & 0 & 3.00 & ,3.00 \\
\hline 42 & 3 & 3 & 3 & 0 & 3.00 & ,3.00 \\
\hline 43 & 3 & 3 & 3 & 0 & 3.00 & ,3.00 \\
\hline 44 & 3 & 3 & 3 & 0 & 3.00 & ,3.00 \\
\hline 45 & 3 & 3 & 3 & 0 & 3.00 & ,3.00 \\
\hline 46 & 2 & 3 & 2.85 & 0.36 & 2.78 & ,2.92 \\
\hline 47 & 2 & 3 & 2.91 & 0.29 & 2.85 & ,2.97 \\
\hline 48 & 2 & 3 & 2.6 & 0.49 & 2.50 & , 2.70 \\
\hline 49 & 1 & 1 & 1 & 0 & 1.00 & ,1.00 \\
\hline 50 & 2 & 2 & 2 & 0 & 2.00 & ,2.00 \\
\hline
\end{tabular}

TABLE A.4: Idle times obtained by applying rotor-router on a random tree with 50 nodes, and 100 distinctive initial configurations for each set of $k=1,2, \ldots, 50$ robots. 


\section{Appendix B}

\section{Rotor-Router Experimental Results for 50 Random Trees}

The experimental results illustrated in Tables B.1, B.2, B.3, and B.4 are obtained by running experiments on the rotor-router simulator for 50 random trees with 50 nodes, and 100 random initial configurations for each set of $k=1,2, \ldots, 50$ robots, i.e., 250, 000 experiments were run in total. 


\begin{tabular}{|c|c|c|c|c|c|c|}
\hline $\mathbf{K}$ & $\begin{array}{l}\text { Minimum } \\
\text { CoverTime }\end{array}$ & $\begin{array}{l}\text { Maximum } \\
\text { CoverTime }\end{array}$ & $\begin{array}{c}\text { Average } \\
\text { CoverTime }\end{array}$ & $\begin{array}{l}\text { Standard } \\
\text { Deviation }\end{array}$ & \multicolumn{2}{|c|}{$\begin{array}{c}\text { 95\% Confidence } \\
\text { Interval }\end{array}$} \\
\hline 1 & 170 & 994 & 445.72 & 59.11 & 444.09 & ,447.36 \\
\hline 2 & 78 & 544 & 218.69 & 25.89 & 217.97 & 219.41 \\
\hline 3 & 47 & 382 & 147.84 & 12.15 & 147.50 & ,148.18 \\
\hline 4 & 40 & 304 & 109.16 & 8.59 & 108.93 & , 109.40 \\
\hline 5 & 26 & 271 & 85.94 & 7.13 & 85.74 & ,86.14 \\
\hline 6 & 24 & 237 & 70.67 & 4.84 & 70.54 & ,70.80 \\
\hline 7 & 20 & 228 & 60.35 & 4.01 & 60.24 &, 60.47 \\
\hline 8 & 16 & 222 & 51.94 & 3.15 & 51.85 & ,52.03 \\
\hline 9 & 14 & 165 & 45.73 & 3.07 & 45.65 & ,45.82 \\
\hline 10 & 12 & 154 & 39.95 & 2.57 & 39.88 & ,40.03 \\
\hline 11 & 10 & 149 & 36.31 & 2.07 & 36.25 & ,36.37 \\
\hline 12 & 9 & 143 & 32.81 & 1.81 & 32.76 & ,32.86 \\
\hline 13 & 10 & 110 & 29.64 & 1.62 & 29.60 & 29.69 \\
\hline 14 & 8 & 93 & 27.14 & 1.67 & 27.10 & ,27.19 \\
\hline 15 & 9 & 91 & 24.95 & 1.31 & 24.92 & 24.99 \\
\hline 16 & 8 & 76 & 23.11 & 1.25 & 23.07 & ,23.14 \\
\hline 17 & 8 & 98 & 21.74 & 1.31 & 21.71 & ,21.78 \\
\hline 18 & 6 & 67 & 20.05 & 0.9 & 20.02 & 20.07 \\
\hline 19 & 6 & 116 & 18.93 & 1.04 & 18.90 & ,18.96 \\
\hline 20 & 6 & 75 & 17.81 & 0.92 & 17.79 & ,17.84 \\
\hline 21 & 5 & 61 & 16.77 & 1 & 16.74 & ,16.79 \\
\hline 22 & 5 & 70 & 15.63 & 0.77 & 15.61 & ,15.65 \\
\hline 23 & 5 & 63 & 14.72 & 0.69 & 14.70 &, 14.74 \\
\hline 24 & 4 & 63 & 14.32 & 0.69 & 14.30 &, 14.34 \\
\hline 25 & 4 & 63 & 13.55 & 0.71 & 13.53 & , 13.57 \\
\hline 26 & 4 & 74 & 12.84 & 0.68 & 12.82 & ,12.86 \\
\hline 27 & 3 & 54 & 12.26 & 0.46 & 12.25 & ,12.27 \\
\hline 28 & 4 & 43 & 11.65 & 0.58 & 11.63 & ,11.66 \\
\hline 29 & 3 & 42 & 11.29 & 0.52 & 11.27 & ,11.30 \\
\hline 30 & 3 & 55 & 10.86 & 0.51 & 10.85 & ,10.88 \\
\hline 31 & 3 & 49 & 10.32 & 0.58 & 10.30 & ,10.34 \\
\hline 32 & 3 & 44 & 9.89 & 0.45 & 9.88 & ,9.90 \\
\hline 33 & 3 & 43 & 9.56 & 0.41 & 9.55 & ,9.57 \\
\hline 34 & 3 & 37 & 9.25 & 0.4 & 9.24 & ,9.26 \\
\hline 35 & 3 & 52 & 8.71 & 0.46 & 8.70 & ,8.73 \\
\hline 36 & 3 & 35 & 8.59 & 0.51 & 8.58 & ,8.61 \\
\hline 37 & 2 & 42 & 8.14 & 0.31 & 8.13 & ,8.14 \\
\hline 38 & 2 & 30 & 7.89 & 0.39 & 7.88 & ,7.90 \\
\hline 39 & 2 & 31 & 7.62 & 0.32 & 7.61 & ,7.63 \\
\hline 40 & 2 & 29 & 7.4 & 0.39 & 7.39 &, 7.41 \\
\hline 41 & 2 & 28 & 7.1 & 0.32 & 7.09 & ,7.11 \\
\hline 42 & 2 & 28 & 6.88 & 0.34 & 6.87 & ,6.89 \\
\hline 43 & 2 & 27 & 6.7 & 0.35 & 6.69 &, 6.71 \\
\hline 44 & 2 & 32 & 6.49 & 0.28 & 6.48 &, 6.50 \\
\hline 45 & 2 & 27 & 6.4 & 0.27 & 6.39 &, 6.41 \\
\hline 46 & 2 & 27 & 6.15 & 0.31 & 6.14 &, 6.16 \\
\hline 47 & 2 & 27 & 5.96 & 0.3 & 5.95 &, 5.97 \\
\hline 48 & 2 & 30 & 5.8 & 0.23 & 5.79 &, 5.80 \\
\hline 49 & 1 & 27 & 5.65 & 0.26 & 5.65 &, 5.66 \\
\hline 50 & 1 & 31 & 5.47 & 0.25 & 5.46 &, 5.47 \\
\hline
\end{tabular}

TABLE B.1: Cover times obtained by applying rotor-router on 50 random trees with 50 nodes, and 100 distinctive initial configurations for each set of $k=1,2, \ldots, 50$ robots. 


\begin{tabular}{|c|c|c|c|c|c|c|}
\hline \multirow{2}{*}{$\begin{array}{r}\mathbf{K} \\
1\end{array}$} & \multirow{2}{*}{$\begin{array}{c}\text { Minimum } \\
\text { Lock-inTime } \\
171\end{array}$} & \multirow{2}{*}{$\begin{array}{c}\text { Maximum } \\
\text { Lock-inTime } \\
995\end{array}$} & \multirow{2}{*}{$\begin{array}{c}\text { Average } \\
\text { Lock-inTime } \\
446.99\end{array}$} & \multirow{2}{*}{$\begin{array}{c}\text { Standard } \\
\text { Deviation } \\
59.16\end{array}$} & \multicolumn{2}{|c|}{$\begin{array}{c}\text { 95\% Confidence } \\
\text { Interval }\end{array}$} \\
\hline & & & & & 445.35 & ,448.63 \\
\hline 2 & 107 & 2486 & 788.63 & 390.92 & 777.80 & ,799.47 \\
\hline 3 & 148 & 2349 & 825.55 & 278.87 & 817.82 & ,833.28 \\
\hline 4 & 120 & 2606 & 853.53 & 264.22 & 846.21 & 860.86 \\
\hline 5 & 163 & 2597 & 896.91 & 261.76 & 889.66 & ,904.17 \\
\hline 6 & 153 & 2435 & 906.65 & 266.56 & 899.26 & ,914.04 \\
\hline 7 & 32 & 1042 & 383.84 & 99.9 & 381.07 & ,386.61 \\
\hline 8 & 151 & 2020 & 879 & 209.66 & 873.19 & ,884.81 \\
\hline 9 & 185 & 2329 & 887.39 & 259.03 & 880.21 & ,894.57 \\
\hline 10 & 159 & 2093 & 896.99 & 220.29 & 890.89 & ,903.10 \\
\hline 11 & 176 & 2201 & 951.71 & 232.62 & 945.27 &, 958.16 \\
\hline 12 & 149 & 1956 & 902.09 & 197 & 896.63 & ,907.55 \\
\hline 13 & 150 & 2493 & 929.94 & 305.15 & 921.48 & ,938.40 \\
\hline 14 & 51 & 1111 & 428.68 & 116.39 & 425.46 & ,431.91 \\
\hline 15 & 211 & 2923 & 958.72 & 253.43 & 951.69 &, 965.74 \\
\hline 16 & 181 & 1877 & 882.09 & 178.41 & 877.14 & ,887.03 \\
\hline 17 & 202 & 2725 & 981 & 338.37 & 971.62 & ,990.37 \\
\hline 18 & 160 & 2265 & 966.58 & 308.27 & 958.03 &, 975.12 \\
\hline 19 & 205 & 2658 & 978.89 & 322.46 & 969.95 & ,987.83 \\
\hline 20 & 176 & 2629 & 943.12 & 353.23 & 933.32 & ,952.91 \\
\hline 21 & 55 & 1194 & 453 & 118.19 & 449.72 & ,456.27 \\
\hline 22 & 162 & 2653 & 1008.76 & 347.86 & 999.12 & ,1018.40 \\
\hline 23 & 167 & 2477 & 981.74 & 307.51 & 973.22 &, 990.27 \\
\hline 24 & 193 & 1709 & 876.4 & 163.65 & 871.86 & ,880.93 \\
\hline 25 & 189 & 1712 & 885.08 & 164 & 880.54 & ,889.63 \\
\hline 26 & 193 & 2471 & 996.56 & 307.54 & 988.03 & ,1005.08 \\
\hline 27 & 176 & 2631 & 1024.99 & 348.6 & 1015.33 & ,1034.65 \\
\hline 28 & 65 & 1191 & 478.87 & 125.85 & 475.38 & ,482.36 \\
\hline 29 & 199 & 2591 & 986.52 & 359.98 & 976.54 & ,996.50 \\
\hline 30 & 211 & 2663 & 1007.48 & 324.31 & 998.50 & ,1016.47 \\
\hline 31 & 203 & 2456 & 1017.74 & 323.98 & 1008.76 & ,1026.72 \\
\hline 32 & 164 & 2672 & 1009.47 & 342 & 999.99 & ,1018.94 \\
\hline 33 & 240 & 1862 & 934.38 & 194.86 & 928.98 & ,939.78 \\
\hline 34 & 196 & 2974 & 1027.41 & 275.54 & 1019.77 & ,1035.04 \\
\hline 35 & 66 & 1211 & 496.18 & 132.6 & 492.51 & ,499.86 \\
\hline 36 & 216 & 2551 & 1002.97 & 334.55 & 993.70 & ,1012.25 \\
\hline 37 & 177 & 2127 & 997.11 & 228.67 & 990.77 &, 1003.45 \\
\hline 38 & 207 & 2359 & 1044.09 & 263.4 & 1036.79 & ,1051.39 \\
\hline 39 & 203 & 2231 & 1008.34 & 259.31 & 1001.15 & ,1015.53 \\
\hline 40 & 195 & 2536 & 1003.42 & 286.79 & 995.47 & ,1011.36 \\
\hline 41 & 221 & 2193 & 1025.95 & 247.66 & 1019.09 & ,1032.82 \\
\hline 42 & 57 & 1278 & 508.97 & 133.66 & 505.27 &, 512.68 \\
\hline 43 & 187 & 2598 & 1089.6 & 301.36 & 1081.24 & ,1097.95 \\
\hline 44 & 182 & 2968 & 1059.52 & 321.52 & 1050.61 & ,1068.43 \\
\hline 45 & 201 & 2781 & 1055.86 & 307.64 & 1047.33 & ,1064.39 \\
\hline 46 & 177 & 2620 & 1050.95 & 352.08 & 1041.19 & ,1060.71 \\
\hline 47 & 196 & 3052 & 1095.16 & 458.84 & 1082.44 & ,1107.88 \\
\hline 48 & 232 & 1650 & 857.76 & 145.39 & 853.73 & ,861.79 \\
\hline 49 & 11 & 762 & 202.45 & 55.87 & 200.90 & 203.99 \\
\hline 50 & 154 & 1694 & 845.47 & 142.21 & 841.53 & ,849.42 \\
\hline
\end{tabular}

TABLE B.2: Lock-in times obtained by applying rotor-router on 50 random trees with 50 nodes, and 100 distinctive initial configurations for each set of $k=1,2, \ldots, 50$ robots. 


\begin{tabular}{|c|c|c|c|c|c|c|}
\hline $\mathrm{K}$ & $\begin{array}{l}\text { Minimum } \\
\text { Periodicity }\end{array}$ & $\begin{array}{l}\text { Maximum } \\
\text { Periodicity }\end{array}$ & $\begin{array}{c}\text { Average } \\
\text { Periodicity }\end{array}$ & $\begin{array}{l}\text { Standard } \\
\text { Deviation }\end{array}$ & \multicolumn{2}{|c|}{$\begin{array}{c}95 \% \text { Confidence } \\
\text { Interval }\end{array}$} \\
\hline 1 & 98 & 98 & 98 & 0 & 98.00 & 98.00 \\
\hline 2 & 49 & 98 & 78.83 & 9.3 & 78.57 & ,79.09 \\
\hline 3 & 98 & 98 & 98 & 0 & 98.00 & ,98.00 \\
\hline 4 & 49 & 98 & 81.08 & 4.01 & 80.96 & ,81.19 \\
\hline 5 & 98 & 98 & 98 & 0 & 98.00 & ,98.00 \\
\hline 6 & 49 & 98 & 83.77 & 2.27 & 83.71 & 83.83, \\
\hline 7 & 14 & 140 & 25.24 & 10.56 & 24.95 & 25.54 \\
\hline 8 & 49 & 98 & 85.14 & 1.8 & 85.09 & ,85.19 \\
\hline 9 & 98 & 98 & 98 & 0 & 98.00 & ,98.00 \\
\hline 10 & 49 & 98 & 86.35 & 2.42 & 86.28 & ,86.41 \\
\hline 11 & 98 & 98 & 98 & 0 & 98.00 & ,98.00 \\
\hline 12 & 49 & 98 & 86.62 & 2.11 & 86.56 & ,86.68 \\
\hline 13 & 98 & 98 & 98 & 0 & 98.00 & ,98.00 \\
\hline 14 & 7 & 140 & 22.33 & 8.4 & 22.10 & 22.56 \\
\hline 15 & 98 & 98 & 98 & 0 & 98.00 & ,98.00 \\
\hline 16 & 49 & 98 & 88.94 & 1.96 & 88.88 & 88.99, \\
\hline 17 & 98 & 98 & 98 & 0 & 98.00 & ,98.00 \\
\hline 18 & 49 & 98 & 90.42 & 2.62 & 90.35 & ,90.50 \\
\hline 19 & 98 & 98 & 98 & 0 & 98.00 & ,98.00 \\
\hline 20 & 49 & 98 & 90.9 & 2.51 & 90.83 & ,90.96 \\
\hline 21 & 14 & 140 & 24.05 & 9.38 & 23.79 & 24.31 \\
\hline 22 & 49 & 98 & 90.95 & 2.74 & 90.88 & ,91.03 \\
\hline 23 & 98 & 98 & 98 & 0 & 98.00 & ,98.00 \\
\hline 24 & 49 & 98 & 90.77 & 1.93 & 90.71 & ,90.82 \\
\hline 25 & 98 & 98 & 98 & 0 & 98.00 & ,98.00 \\
\hline 26 & 49 & 98 & 91.2 & 2.16 & 91.14 & ,91.26 \\
\hline 27 & 98 & 98 & 98 & 0 & 98.00 & ,98.00 \\
\hline 28 & 7 & 84 & 22.9 & 8.77 & 22.66 & ,23.14 \\
\hline 29 & 98 & 98 & 98 & 0 & 98.00 & ,98.00 \\
\hline 30 & 49 & 98 & 92.35 & 2.29 & 92.28 & ,92.41 \\
\hline 31 & 98 & 98 & 98 & 0 & 98.00 & ,98.00 \\
\hline 32 & 49 & 98 & 92.32 & 2.37 & 92.25 &, 92.38 \\
\hline 33 & 98 & 98 & 98 & 0 & 98.00 & ,98.00 \\
\hline 34 & 49 & 98 & 92.26 & 1.77 & 92.21 & ,92.31 \\
\hline 35 & 14 & 140 & 24 & 9.38 & 23.74 & ,24.26 \\
\hline 36 & 49 & 98 & 92.84 & 2.01 & 92.78 & ,92.89 \\
\hline 37 & 98 & 98 & 98 & 0 & 98.00 & ,98.00 \\
\hline 38 & 49 & 98 & 91.96 & 1.64 & 91.92 & ,92.01 \\
\hline 39 & 98 & 98 & 98 & 0 & 98.00 & ,98.00 \\
\hline 40 & 49 & 98 & 92.6 & 1.87 & 92.55 & ,92.65 \\
\hline 41 & 98 & 98 & 98 & 0 & 98.00 & ,98.00 \\
\hline 42 & 7 & 84 & 23.33 & 8.99 & 23.08 & 23.58 \\
\hline 43 & 98 & 98 & 98 & 0 & 98.00 & ,98.00 \\
\hline 44 & 49 & 98 & 92.79 & 1.6 & 92.74 & ,92.83 \\
\hline 45 & 98 & 98 & 98 & 0 & 98.00 & ,98.00 \\
\hline 46 & 49 & 98 & 93.29 & 2.01 & 93.23 & ,93.34 \\
\hline 47 & 98 & 98 & 98 & 0 & 98.00 &, 98.00 \\
\hline 48 & 49 & 98 & 92.85 & 1.62 & 92.80 & ,92.89 \\
\hline 49 & 2 & 2 & 2 & 0 & 2.00 & ,2.00 \\
\hline 50 & 49 & 98 & 92.64 & 1.68 & 92.59 & ,92.69 \\
\hline
\end{tabular}

TABLE B.3: Periodicities obtained by applying rotor-router on 50 random trees with 50 nodes, and 100 distinctive initial configurations for each set of $k=1,2, \ldots, 50$ robots. 


\begin{tabular}{|c|c|c|c|c|c|c|}
\hline $\mathbf{K}$ & $\begin{array}{l}\text { Minimum } \\
\text { IdleTime } \\
\end{array}$ & $\begin{array}{l}\text { Maximum } \\
\text { IdleTime }\end{array}$ & $\begin{array}{l}\text { Average } \\
\text { IdleTime }\end{array}$ & $\begin{array}{l}\text { Standard } \\
\text { Deviation }\end{array}$ & \multicolumn{2}{|c|}{$\begin{array}{c}\text { 95\% Confidence } \\
\text { Interval }\end{array}$} \\
\hline 1 & 97 & 97 & 97 & 0 & 97.00 & ,97.00 \\
\hline 2 & 48 & 63 & 49.42 & 1.36 & 49.38 & ,49.46 \\
\hline 3 & 32 & 41 & 32.79 & 0.41 & 32.78 & ,32.81 \\
\hline 4 & 24 & 28 & 24.6 & 0.3 & 24.59 & 24.61 \\
\hline 5 & 19 & 21 & 19.33 & 0.31 & 19.32 & ,19.34 \\
\hline 6 & 16 & 17 & 16.54 & 0.27 & 16.54 & , 16.55 \\
\hline 7 & 13 & 17 & 13.47 & 0.27 & 13.46 & ,13.48 \\
\hline 8 & 12 & 13 & 12.49 & 0.27 & 12.48 & ,12.50 \\
\hline 9 & 10 & 12 & 10.99 & 0.07 & 10.99 & ,10.99 \\
\hline 10 & 9 & 10 & 9.9 & 0.25 & 9.90 & ,9.91 \\
\hline 11 & 9 & 10 & 9 & 0.01 & 9.00 & ,9.00 \\
\hline 12 & 8 & 9 & 8.48 & 0.24 & 8.47 & ,8.49 \\
\hline 13 & 7 & 8 & 7.87 & 0.14 & 7.86 & ,7.87 \\
\hline 14 & 7 & 8 & 7 & 0.01 & 7.00 & ,7.00 \\
\hline 15 & 7 & 7 & 7 & 0 & 7.00 & ,7.00 \\
\hline 16 & 6 & 7 & 6.44 & 0.25 & 6.43 & ,6.45 \\
\hline 17 & 5 & 6 & 6 & 0 & 6.00 & ,6.00 \\
\hline 18 & 5 & 6 & 5.9 & 0.03 & 5.90 & ,5.90 \\
\hline 19 & 5 & 6 & 5.16 & 0.16 & 5.15 &, 5.16 \\
\hline 20 & 5 & 6 & 5 & 0 & 5.00 & ,5.00 \\
\hline 21 & 5 & 5 & 5 & 0 & 5.00 &, 5.00 \\
\hline 22 & 5 & 5 & 5 & 0 & 5.00 &, 5.00 \\
\hline 23 & 4 & 5 & 4.98 & 0.11 & 4.98 & ,4.99 \\
\hline 24 & 4 & 5 & 4.44 & 0.25 & 4.43 & ,4.45 \\
\hline 25 & 4 & 4 & 4 & 0 & 4.00 & ,4.00 \\
\hline 26 & 3 & 4 & 4 & 0 & 4.00 & ,4.00 \\
\hline 27 & 3 & 4 & 4 & 0.01 & 4.00 & ,4.00 \\
\hline 28 & 3 & 4 & 3.95 & 0.07 & 3.95 & ,3.96 \\
\hline 29 & 3 & 4 & 3.92 & 0.03 & 3.92 & ,3.92 \\
\hline 30 & 3 & 4 & 3.77 & 0.05 & 3.77 & ,3.78 \\
\hline 31 & 3 & 4 & 3.43 & 0.2 & 3.43 & ,3.44 \\
\hline 32 & 3 & 4 & 3.05 & 0.07 & 3.05 & ,3.06 \\
\hline 33 & 3 & 3 & 3 & 0 & 3.00 & ,3.00 \\
\hline 34 & 3 & 3 & 3 & 0 & 3.00 & ,3.00 \\
\hline 35 & 3 & 3 & 3 & 0 & 3.00 & ,3.00 \\
\hline 36 & 3 & 3 & 3 & 0 & 3.00 & ,3.00 \\
\hline 37 & 3 & 3 & 3 & 0 & 3.00 & ,3.00 \\
\hline 38 & 3 & 3 & 3 & 0 & 3.00 & ,3.00 \\
\hline 39 & 3 & 3 & 3 & 0 & 3.00 & ,3.00 \\
\hline 40 & 3 & 3 & 3 & 0 & 3.00 & ,3.00 \\
\hline 41 & 3 & 3 & 3 & 0 & 3.00 & ,3.00 \\
\hline 42 & 3 & 3 & 3 & 0 & 3.00 & ,3.00 \\
\hline 43 & 3 & 3 & 3 & 0 & 3.00 & ,3.00 \\
\hline 44 & 2 & 3 & 2.99 & 0.04 & 2.99 & ,3.00 \\
\hline 45 & 2 & 3 & 3 & 0.02 & 3.00 & ,3.00 \\
\hline 46 & 2 & 3 & 2.97 & 0.1 & 2.97 & ,2.97 \\
\hline 47 & 2 & 3 & 2.89 & 0.17 & 2.88 & ,2.89 \\
\hline 48 & 2 & 3 & 2.46 & 0.24 & 2.46 & ,2.47 \\
\hline 49 & 1 & 1 & 1 & 0 & 1.00 & , 1.00 \\
\hline 50 & 2 & 2 & 2 & 0 & 2.00 & ,2.00 \\
\hline
\end{tabular}

TABLE B.4: Idle times obtained by applying rotor-router on 50 random trees with 50 nodes, and 100 distinctive initial configurations for each set of $k=1,2, \ldots, 50$ robots. 


\section{Appendix C}

\section{Rotor-Router Experimental Results for a Random Clover Tree}

The experimental results illustrated in Tables C.1, C.2, C.3, C.4, C.5, C.6, C.7, and C.8 are obtained by running experiments on the rotor-router simulator for a random Clover tree with 106 nodes, and 100 random initial configurations for each set of $k=1,2, \ldots, 105$ robots, i.e., 10, 500 experiments were run in total. For the sake of clarity, we display the results obtained for each of the measurement parameters (cover time, lock-in time, periodicity, and idle time) in two separated tables: one for $k=1, \ldots, 52$, and another for $k=53, \ldots, 105$. 


\begin{tabular}{|c|c|c|c|c|c|c|}
\hline \multirow[t]{2}{*}{$\mathrm{K}$} & \multirow{2}{*}{$\begin{array}{l}\text { Minimum } \\
\text { CoverTime }\end{array}$} & \multirow{2}{*}{$\begin{array}{l}\text { Maximum } \\
\text { CoverTime }\end{array}$} & \multirow{2}{*}{$\begin{array}{c}\text { Average } \\
\text { CoverTime }\end{array}$} & \multirow{2}{*}{$\begin{array}{l}\text { Standard } \\
\text { Deviation }\end{array}$} & \multicolumn{2}{|c|}{$95 \%$ Confidence } \\
\hline & & & & & & \\
\hline 1 & 634 & 1635 & 1084.01 & 214.42 & 1041.98 & ,1126.04 \\
\hline 2 & 327 & 1035 & 642.75 & 136.23 & 616.05 &, 669.45 \\
\hline 3 & 183 & 683 & 393.98 & 111.95 & 372.04 & ,415.92 \\
\hline 4 & 146 & 483 & 290.24 & 82.16 & 274.14 & ,306.34 \\
\hline 5 & 111 & 444 & 238.15 & 75.27 & 223.40 & ,252.90 \\
\hline $\mathrm{K}$ & 93 & 403 & 207.3 & 65.47 & 194.47 & ,220.13 \\
\hline 6 & 85 & 322 & 164.3 & 58.23 & 152.89 & ,175.71 \\
\hline 7 & 79 & 324 & 153.13 & 57.35 & 141.89 & ,164.37 \\
\hline 8 & 74 & 276 & 135.28 & 47.62 & 125.95 & ,144.61 \\
\hline 9 & 52 & 201 & 112.26 & 34.54 & 105.49 & ,119.03 \\
\hline 10 & 47 & 231 & 101.97 & 34.51 & 95.21 & , 108.73 \\
\hline $\mathrm{K}$ & 48 & 173 & 101.25 & 31.66 & 95.04 &, 107.46 \\
\hline 11 & 38 & 207 & 86.54 & 30.61 & 80.54 &, 92.54 \\
\hline 12 & 34 & 164 & 79.6 & 25.93 & 74.52 & ,84.68 \\
\hline 13 & 40 & 212 & 73.83 & 29.84 & 67.98 &, 79.68 \\
\hline 14 & 37 & 152 & 71.13 & 22.92 & 66.64 & ,75.62 \\
\hline 15 & 28 & 159 & 71.23 & 27.98 & 65.75 &, 76.71 \\
\hline $\mathrm{K}$ & 26 & 157 & 61.13 & 20.72 & 57.07 &, 65.19 \\
\hline 16 & 31 & 186 & 60.65 & 24.51 & 55.85 &, 65.45 \\
\hline 17 & 27 & 112 & 54.95 & 16.47 & 51.72 &, 58.18 \\
\hline 18 & 25 & 134 & 52.23 & 18.76 & 48.55 &, 55.91 \\
\hline 19 & 24 & 105 & 49.81 & 16.1 & 46.65 &, 52.97 \\
\hline 20 & 23 & 114 & 47.96 & 15.75 & 44.87 &, 51.05 \\
\hline 24 & 20 & 98 & 44.67 & 14.9 & 41.75 & ,47.59 \\
\hline 25 & 22 & 79 & 39.24 & 10.58 & 37.17 &, 41.31 \\
\hline 26 & 21 & 70 & 39.44 & 11.09 & 37.27 &, 41.61 \\
\hline 27 & 18 & 104 & 41.53 & 15.15 & 38.56 &, 44.50 \\
\hline 28 & 19 & 75 & 35.56 & 10.3 & 33.54 & ,37.58 \\
\hline 29 & 19 & 79 & 37.4 & 12.2 & 35.01 & ,39.79 \\
\hline 30 & 16 & 80 & 33.4 & 11.4 & 31.17 & ,35.63 \\
\hline 31 & 19 & 82 & 34.4 & 9.42 & 32.55 &, 36.25 \\
\hline 32 & 15 & 63 & 30.2 & 9.68 & 28.30 &, 32.10 \\
\hline 33 & 13 & 78 & 30.93 & 10.7 & 28.83 & ,33.03 \\
\hline 34 & 13 & 106 & 29.38 & 10.98 & 27.23 &, 31.53 \\
\hline 35 & 12 & 51 & 26.19 & 7.27 & 24.76 & ,27.62 \\
\hline 36 & 14 & 77 & 27.18 & 10.73 & 25.08 & ,29.28 \\
\hline 37 & 11 & 61 & 28.36 & 9.94 & 26.41 &, 30.31 \\
\hline 38 & 13 & 54 & 25.43 & 7.55 & 23.95 & ,26.91 \\
\hline 39 & 10 & 50 & 24.69 & 8.1 & 23.10 & ,26.28 \\
\hline 40 & 13 & 74 & 25.09 & 9.42 & 23.24 & ,26.94 \\
\hline 41 & 12 & 51 & 22.39 & 7.48 & 20.92 & ,23.86 \\
\hline 42 & 10 & 59 & 22.24 & 7.52 & 20.77 & ,23.71 \\
\hline 43 & 11 & 50 & 22.42 & 7.12 & 21.02 & ,23.82 \\
\hline 44 & 10 & 49 & 21.45 & 7.09 & 20.06 & ,22.84 \\
\hline 45 & 10 & 43 & 20.91 & 7.02 & 19.53 & ,22.29 \\
\hline 46 & 9 & 40 & 20.6 & 6.43 & 19.34 & ,21.86 \\
\hline 47 & 9 & 58 & 19.98 & 8.13 & 18.39 & ,21.57 \\
\hline 48 & 9 & 38 & 18.51 & 6.71 & 17.19 &, 19.83 \\
\hline 49 & 8 & 38 & 18.74 & 6.37 & 17.49 &, 19.99 \\
\hline 50 & 10 & 37 & 18.29 & 5.59 & 17.19 & ,19.39 \\
\hline 51 & 7 & 43 & 18.54 & 6.17 & 17.33 & ,19.75 \\
\hline 52 & 9 & 38 & 18.7 & 6 & 17.52 & ,19.88 \\
\hline
\end{tabular}

TABLE C.1: Cover times obtained by applying rotor-router on a random Clover tree with 106 nodes, and 100 random initial configurations for each set of $k=1, \ldots, 52$. 


\begin{tabular}{|c|c|c|c|c|c|c|}
\hline $\mathrm{K}$ & $\begin{array}{l}\text { Minimum } \\
\text { CoverTime }\end{array}$ & $\begin{array}{l}\text { Maximum } \\
\text { CoverTime }\end{array}$ & $\begin{array}{c}\text { Average } \\
\text { CoverTime }\end{array}$ & $\begin{array}{l}\text { Standard } \\
\text { Deviation }\end{array}$ & \multicolumn{2}{|c|}{$\begin{array}{c}\text { 95\% Confidence } \\
\text { Interval }\end{array}$} \\
\hline 53 & 10 & 34 & 16.52 & 4.83 & 15.57 &, 17.47 \\
\hline 54 & 9 & 37 & 17.24 & 5.69 & 16.12 & ,18.36 \\
\hline 55 & 8 & 71 & 16.36 & 7.71 & 14.85 & 17.87 \\
\hline 56 & 8 & 38 & 16.5 & 6.12 & 15.30 & , 17.70 \\
\hline 57 & 7 & 43 & 14.68 & 5.67 & 13.57 & 15.79 \\
\hline 58 & 9 & 30 & 15.94 & 4.88 & 14.98 & 16.90 \\
\hline 59 & 7 & 31 & 14.72 & 4.43 & 13.85 & ,15.59 \\
\hline 60 & 6 & 29 & 14.61 & 5.13 & 13.61 &, 15.61 \\
\hline 61 & 6 & 38 & 14.73 & 5.37 & 13.68 & ,15.78 \\
\hline 62 & 7 & 36 & 15.08 & 5.45 & 14.01 & ,16.15 \\
\hline 63 & 6 & 32 & 13.79 & 5.19 & 12.77 & ,14.81 \\
\hline 64 & 6 & 26 & 12.72 & 4.2 & 11.90 & ,13.54 \\
\hline 65 & 6 & 37 & 13.28 & 5 & 12.30 & ,14.26 \\
\hline 66 & 6 & 41 & 13.69 & 5.12 & 12.69 & 14.69 \\
\hline 67 & 5 & 28 & 12.7 & 4.47 & 11.82 & ,13.58 \\
\hline 68 & 5 & 26 & 12.27 & 3.67 & 11.55 & ,12.99 \\
\hline 69 & 6 & 30 & 12.14 & 4.05 & 11.35 &, 12.93 \\
\hline 70 & 6 & 27 & 12.37 & 4.38 & 11.51 & ,13.23 \\
\hline 71 & 5 & 40 & 12.64 & 5.32 & 11.60 & ,13.68 \\
\hline 72 & 6 & 25 & 12.47 & 4.5 & 11.59 & ,13.35 \\
\hline 73 & 4 & 39 & 11.89 & 5.33 & 10.85 & ,12.93 \\
\hline 74 & 5 & 24 & 11.73 & 4.29 & 10.89 & ,12.57 \\
\hline 75 & 6 & 22 & 11.14 & 3.7 & 10.41 & 11.87 \\
\hline 76 & 4 & 21 & 10.67 & 3.66 & 9.95 & 11.39 \\
\hline 77 & 4 & 23 & 10.28 & 3.34 & 9.62 & 10.94 \\
\hline 78 & 4 & 20 & 10.2 & 3.27 & 9.56 & 10.84 \\
\hline 79 & 5 & 21 & 10.06 & 3.54 & 9.37 & 10.75 \\
\hline 80 & 5 & 22 & 10.06 & 3.85 & 9.31 & 10.81 \\
\hline 81 & 5 & 23 & 9.68 & 3.58 & 8.98 & , 10.38 \\
\hline 82 & 3 & 26 & 9.61 & 3.66 & 8.89 & , 10.33 \\
\hline 83 & 5 & 27 & 10.32 & 4.04 & 9.53 & ,11.11 \\
\hline 84 & 5 & 23 & 9.72 & 3.22 & 9.09 & ,10.35 \\
\hline 85 & 5 & 23 & 9.59 & 3.37 & 8.93 & 10.25 \\
\hline 86 & 4 & 20 & 8.98 & 3.04 & 8.38 & ,9.58 \\
\hline 87 & 3 & 17 & 8.79 & 2.61 & 8.28 & 9.30 \\
\hline 88 & 4 & 26 & 9.04 & 3.75 & 8.30 & ,9.78 \\
\hline 89 & 5 & 22 & 8.68 & 3.65 & 7.96 &, 9.40 \\
\hline 90 & 4 & 23 & 8.86 & 3.46 & 8.18 &, 9.54 \\
\hline 91 & 4 & 16 & 8.42 & 2.91 & 7.85 & ,8.99 \\
\hline 92 & 4 & 20 & 8.59 & 3.45 & 7.91 &, 9.27 \\
\hline 93 & 4 & 20 & 8.43 & 3.36 & 7.77 & 9.09 \\
\hline 94 & 4 & 21 & 8.21 & 3.35 & 7.55 & 8.87 \\
\hline 95 & 4 & 16 & 8.25 & 2.75 & 7.71 & 8.79 \\
\hline 96 & 4 & 18 & 8.28 & 3.12 & 7.67 & ,8.89 \\
\hline 97 & 4 & 23 & 8.23 & 3.31 & 7.58 & ,8.88 \\
\hline 98 & 4 & 23 & 7.85 & 3.61 & 7.14 & ,8.56 \\
\hline 99 & 3 & 25 & 8.44 & 3.66 & 7.72 &, 9.16 \\
\hline 100 & 3 & 22 & 7.84 & 3.1 & 7.23 & ,8.45 \\
\hline 101 & 3 & 16 & 7.35 & 2.68 & 6.82 & ,7.88 \\
\hline 102 & 3 & 26 & 7.08 & 2.97 & 6.50 & ,7.66 \\
\hline 103 & 4 & 19 & 7.21 & 2.61 & 6.70 & ,7.72 \\
\hline 104 & 3 & 16 & 6.84 & 2.71 & 6.31 & ,7.37 \\
\hline 105 & 3 & 14 & 6.98 & 2.34 & 6.52 &, 7.44 \\
\hline
\end{tabular}

TABLE C.2: Cover times obtained by applying rotor-router on a random Clover tree with 106 nodes, and 100 random initial configurations for each set of $k=53, \ldots, 105$. 


\begin{tabular}{|c|c|c|c|c|c|c|}
\hline $\mathrm{K}$ & $\begin{array}{c}\text { Minimum } \\
\text { Lock-inTime }\end{array}$ & $\begin{array}{l}\text { Maximum } \\
\text { Lock-inTime }\end{array}$ & $\begin{array}{c}\text { Average } \\
\text { Lock-inTime }\end{array}$ & $\begin{array}{l}\text { Standard } \\
\text { Deviation }\end{array}$ & $\begin{array}{r}95 \% \mathrm{C} \\
\text { Int }\end{array}$ & $\begin{array}{l}\text { fidence } \\
\text { val }\end{array}$ \\
\hline 1 & 636 & 1636 & 1085.13 & 214.38 & 1043.11 & ,1127.15 \\
\hline 2 & 437 & 1138 & 766.42 & 136.84 & 739.60 & ,793.24 \\
\hline 3 & 263 & 3620 & 2361.75 & 750.38 & 2214.68 & ,2508.82 \\
\hline 4 & 513 & 4352 & 1523.51 & 738.3 & 1378.80 & ,1668.22 \\
\hline 5 & 391 & 1895 & 1188.28 & 343.76 & 1120.90 & ,1255.66 \\
\hline 6 & 652 & 2683 & 1886.91 & 400.32 & 1808.45 & ,1965.37 \\
\hline 7 & 502 & 1975 & 1252.02 & 323.9 & 1188.54 & ,1315.50 \\
\hline 8 & 699 & 3470 & 1673.9 & 623.02 & 1551.79 & ,1796.01 \\
\hline 9 & 799 & 2955 & 1887.21 & 521.91 & 1784.92 & , 1989.50 \\
\hline 10 & 860 & 2738 & 1779.12 & 448.21 & 1691.27 & ,1866.97 \\
\hline 11 & 845 & 3349 & 1826.48 & 557.99 & 1717.11 & ,1935.85 \\
\hline 12 & 420 & 2323 & 1479.36 & 389.76 & 1402.97 & ,1555.75 \\
\hline 13 & 967 & 4330 & 2992.19 & 700.73 & 2854.85 & ,3129.53 \\
\hline 14 & 613 & 1971 & 1406.19 & 263.38 & 1354.57 & ,1457.81 \\
\hline 15 & 218 & 1383 & 950.27 & 218.67 & 907.41 & ,993.13 \\
\hline 16 & 1245 & 3154 & 2383.52 & 403.68 & 2304.40 & ,2462.64 \\
\hline 17 & 724 & 3368 & 1992.18 & 665.19 & 1861.80 & ,2122.56 \\
\hline 18 & 541 & 2059 & 1414.86 & 276.08 & 1360.75 & ,1468.97 \\
\hline 19 & 1278 & 5169 & 3308.99 & 771.66 & 3157.75 & ,3460.23 \\
\hline 20 & 426 & 1753 & 1314.37 & 244.55 & 1266.44 & ,1362.30 \\
\hline 21 & 427 & 1360 & 954.82 & 201.19 & 915.39 &, 994.25 \\
\hline 22 & 960 & 3314 & 2291.53 & 696.44 & 2155.03 & ,2428.03 \\
\hline 23 & 946 & 6997 & 4301.7 & 2032.25 & 3903.38 & ,4700.02 \\
\hline 24 & 236 & 2366 & 1523.44 & 360.59 & 1452.77 & ,1594.11 \\
\hline 25 & 1003 & 2250 & 1624.24 & 266.04 & 1572.10 & ,1676.38 \\
\hline 26 & 1273 & 3187 & 2309.88 & 425.52 & 2226.48 & ,2393.28 \\
\hline 27 & 682 & 2700 & 1496.34 & 497.22 & 1398.89 & ,1593.79 \\
\hline 28 & 882 & 2168 & 1712.84 & 227.61 & 1668.23 & , 1757.45 \\
\hline 29 & 1411 & 5085 & 2251.45 & 732.6 & 2107.86 & ,2395.04 \\
\hline 30 & 332 & 1386 & 1018.06 & 183.35 & 982.12 & 1054.00 \\
\hline 31 & 1383 & 3270 & 2459.62 & 378.39 & 2385.46 & ,2533.78 \\
\hline 32 & 1667 & 4097 & 2810.63 & 702.94 & 2672.85 & ,2948.41 \\
\hline 33 & 438 & 2159 & 1415.51 & 350.68 & 1346.78 & ,1484.24 \\
\hline 34 & 3316 & 6837 & 5017.8 & 1009.52 & 4819.93 &, 5215.67 \\
\hline 35 & 106 & 1098 & 767.76 & 197.29 & 729.09 & 806.43 \\
\hline 36 & 528 & 3040 & 1747.13 & 643.15 & 1621.07 & ,1873.19 \\
\hline 37 & 2242 & 5935 & 3736.7 & 845.93 & 3570.90 & ,3902.50 \\
\hline 38 & 1391 & 3459 & 2217.05 & 445.21 & 2129.79 & ,2304.31 \\
\hline 39 & 718 & 2495 & 1526.88 & 325.09 & 1463.16 & ,1590.60 \\
\hline 40 & 1028 & 2736 & 1962.45 & 294.87 & 1904.66 & ,2020.24 \\
\hline 41 & 1004 & 3678 & 2223.23 & 653.72 & 2095.10 & ,2351.36 \\
\hline 42 & 401 & 1440 & 1054.65 & 210 & 1013.49 & ,1095.81 \\
\hline 43 & 1306 & 4627 & 2965.96 & 799.18 & 2809.32 & ,3122.60 \\
\hline 44 & 870 & 5399 & 2933.22 & 1295.72 & 2679.26 & ,3187.18 \\
\hline 45 & 246 & 1430 & 1055.54 & 214.8 & 1013.44 & ,1097.64 \\
\hline 46 & 1197 & 4302 & 2930.59 & 1019 & 2730.87 & ,3130.31 \\
\hline 47 & 1043 & 2524 & 1803.67 & 339.29 & 1737.17 & , 1870.17 \\
\hline 48 & 517 & 3093 & 2001.87 & 557.32 & 1892.63 & ,2111.11 \\
\hline 49 & 734 & 2476 & 1722.74 & 361.17 & 1651.95 & ,1793.53 \\
\hline 50 & 829 & 2266 & 1629.28 & 266.7 & 1577.01 & ,1681.55 \\
\hline 51 & 920 & 3457 & 2288.87 & 579.11 & 2175.37 & ,2402.37 \\
\hline 52 & 1386 & 2801 & 2210.41 & 334.67 & 2144.82 & ,2276.00 \\
\hline
\end{tabular}

TABLE C.3: Lock-in times obtained by applying rotor-router on a random Clover tree with 106 nodes, and 100 random initial configurations for each set of $k=1, \ldots, 52$. 


\begin{tabular}{|c|c|c|c|c|c|c|}
\hline $\mathrm{K}$ & $\begin{array}{c}\text { Minimum } \\
\text { Lock-inTime }\end{array}$ & $\begin{array}{c}\text { Maximum } \\
\text { Lock-inTime }\end{array}$ & $\begin{array}{c}\text { Average } \\
\text { Lock-inTime }\end{array}$ & $\begin{array}{l}\text { Standard } \\
\text { Deviation }\end{array}$ & \multicolumn{2}{|c|}{$\begin{array}{c}95 \% \text { Confidence } \\
\text { Interval }\end{array}$} \\
\hline 53 & 1127 & 2845 & 2268.04 & 291.35 & 2210.94 & ,2325.14 \\
\hline 54 & 808 & 3282 & 2236.47 & 543.33 & 2129.98 & ,2342.96 \\
\hline 55 & 510 & 2073 & 1602.02 & 293.18 & 1544.56 & ,1659.48 \\
\hline 56 & 505 & 2525 & 1752.9 & 340.41 & 1686.18 & ,1819.62 \\
\hline 57 & 963 & 3074 & 2057.5 & 573.08 & 1945.18 & ,2169.82 \\
\hline 58 & 921 & 2598 & 1847.92 & 350.08 & 1779.30 & ,1916.54 \\
\hline 59 & 1310 & 4145 & 2858.8 & 983.99 & 2665.94 &, 3051.66 \\
\hline 60 & 214 & 1478 & 1124.85 & 225.97 & 1080.56 &, 1169.14 \\
\hline 61 & 947 & 5645 & 3005.98 & 1278.07 & 2755.48 & ,3256.48 \\
\hline 62 & 1462 & 4935 & 3257.2 & 877.05 & 3085.30 & ,3429.10 \\
\hline 63 & 615 & 1443 & 1095.56 & 180.61 & 1060.16 &, 1130.96 \\
\hline 64 & 1049 & 3695 & 2290.44 & 654.23 & 2162.21 & ,2418.67 \\
\hline 65 & 1322 & 2894 & 2102.94 & 309.32 & 2042.31 & ,2163.57 \\
\hline 66 & 676 & 2513 & 1599.66 & 299.99 & 1540.86 & ,1658.46 \\
\hline 67 & 1497 & 3594 & 2190.56 & 434.92 & 2105.32 & ,2275.80 \\
\hline 68 & 2460 & 6300 & 4055.48 & 961.31 & 3867.06 & ,4243.90 \\
\hline 69 & 816 & 3133 & 1879.4 & 632.38 & 1755.45 & ,2003.35 \\
\hline 70 & 146 & 1203 & 845.5 & 201.06 & 806.09 & ,884.91 \\
\hline 71 & 2440 & 6963 & 5204 & 1084.19 & 4991.50 &, 5416.50 \\
\hline 72 & 948 & 2319 & 1652.45 & 328.37 & 1588.09 & ,1716.81 \\
\hline 73 & 1616 & 4146 & 2890.83 & 743.26 & 2745.15 &, 3036.51 \\
\hline 74 & 1438 & 3577 & 2613.56 & 391.35 & 2536.86 & ,2690.26 \\
\hline 75 & 398 & 1593 & 1151 & 237.99 & 1104.35 & ,1197.65 \\
\hline 76 & 1186 & 5093 & 2545.81 & 897.34 & 2369.93 & ,2721.69 \\
\hline 77 & 1331 & 2445 & 1922.19 & 252.45 & 1872.71 &, 1971.67 \\
\hline 78 & 817 & 2887 & 1752.57 & 558.79 & 1643.05 & ,1862.09 \\
\hline 79 & 1692 & 3321 & 2532.84 & 322.77 & 2469.58 & ,2596.10 \\
\hline 80 & 1287 & 2377 & 1843.06 & 245.52 & 1794.94 & ,1891.18 \\
\hline 81 & 905 & 2561 & 1698.22 & 365.48 & 1626.59 & ,1769.85 \\
\hline 82 & 1256 & 7171 & 4134.02 & 2147.54 & 3713.10 & ,4554.94 \\
\hline 83 & 1325 & 3664 & 2483.89 & 693.01 & 2348.06 & 2619.72 \\
\hline 84 & 475 & 1538 & 1110.14 & 202 & 1070.55 & ,1149.73 \\
\hline 85 & 632 & 2073 & 1516.27 & 265.55 & 1464.22 & ,1568.32 \\
\hline 86 & 2052 & 4841 & 3597.95 & 773.54 & 3446.34 & ,3749.56 \\
\hline 87 & 944 & 2226 & 1647.29 & 270.79 & 1594.22 & , 1700.36 \\
\hline 88 & 854 & 3737 & 2141.8 & 792.93 & 1986.38 & ,2297.22 \\
\hline 89 & 1319 & 3242 & 2679.94 & 420.5 & 2597.52 & ,2762.36 \\
\hline 90 & 556 & 1732 & 1207.27 & 212.2 & 1165.68 & ,1248.86 \\
\hline 91 & 540 & 2259 & 1626.56 & 271.62 & 1573.32 & ,1679.80 \\
\hline 92 & 1267 & 4664 & 3212.92 & 782.95 & 3059.46 & ,3366.38 \\
\hline 93 & 1006 & 2596 & 1747.77 & 346.38 & 1679.88 & , 1815.66 \\
\hline 94 & 754 & 3426 & 2087.49 & 569.56 & 1975.86 & ,2199.12 \\
\hline 95 & 1453 & 3034 & 2160.69 & 422.12 & 2077.95 & ,2243.43 \\
\hline 96 & 1155 & 3293 & 2158.14 & 509.59 & 2058.26 & ,2258.02 \\
\hline 97 & 888 & 3627 & 1934.21 & 587.83 & 1818.99 & ,2049.43 \\
\hline 98 & 809 & 2483 & 1687.26 & 332.89 & 1622.01 & ,1752.51 \\
\hline 99 & 1159 & 3209 & 2384.83 & 400.98 & 2306.24 & ,2463.42 \\
\hline 100 & 639 & 2354 & 1596.33 & 398.56 & 1518.21 & ,1674.45 \\
\hline 101 & 1054 & 5231 & 2107.75 & 715.67 & 1967.48 & ,2248.02 \\
\hline 102 & 1441 & 4082 & 2974.71 & 525.12 & 2871.79 & ,3077.63 \\
\hline 103 & 771 & 1947 & 1515.49 & 214.01 & 1473.54 & ,1557.44 \\
\hline 104 & 1313 & 2766 & 2187.44 & 317.21 & 2125.27 & ,2249.61 \\
\hline 105 & 94 & 983 & 578.56 & 173.9 & 544.48 &, 612.64 \\
\hline
\end{tabular}

TABLE C.4: Lock-in times obtained by applying rotor-router on a random Clover tree with 106 nodes, and 100 random initial configurations for each set of $k=53, \ldots, 105$. 


\begin{tabular}{|c|c|c|c|c|c|c|}
\hline $\mathrm{K}$ & $\begin{array}{l}\text { Minimum } \\
\text { Periodicity }\end{array}$ & $\begin{array}{l}\text { Maximum } \\
\text { Periodicity }\end{array}$ & $\begin{array}{l}\text { Average } \\
\text { Periodicity }\end{array}$ & $\begin{array}{l}\text { Standard } \\
\text { Deviation }\end{array}$ & $\begin{array}{r}95 \% \mathrm{C} \\
\ln \end{array}$ & $\begin{array}{l}\text { idence } \\
\text { val }\end{array}$ \\
\hline 1 & 210 & 210 & 210 & 0 & 210.00 & ,210.00 \\
\hline 2 & 105 & 210 & 208.95 & 10.5 & 206.89 & ,211.01 \\
\hline 3 & 70 & 70 & 70 & 0 & 70.00 &, 70.00 \\
\hline 4 & 105 & 210 & 193.2 & 38.69 & 185.62 & 200.78 \\
\hline 5 & 42 & 168 & 159.18 & 30.61 & 153.18 & , 165.18 \\
\hline 6 & 35 & 70 & 60.9 & 15.43 & 57.88 &, 63.92 \\
\hline 7 & 30 & 300 & 207.9 & 54.15 & 197.29 & ,218.51 \\
\hline 8 & 105 & 210 & 181.65 & 46.85 & 172.47 & ,190.83 \\
\hline 9 & 70 & 70 & 70 & 0 & 70.00 &, 70.00 \\
\hline 10 & 21 & 168 & 148.68 & 48.28 & 139.22 & ,158.14 \\
\hline 11 & 210 & 210 & 210 & 0 & 210.00 &, 210.00 \\
\hline 12 & 35 & 70 & 64.75 & 12.56 & 62.29 &, 67.21 \\
\hline 13 & 210 & 210 & 210 & 0 & 210.00 & ,210.00 \\
\hline 14 & 30 & 300 & 193.35 & 58.73 & 181.84 & ,204.86 \\
\hline 15 & 14 & 84 & 28.42 & 16.34 & 25.22 & ,31.62 \\
\hline 16 & 105 & 210 & 200.55 & 30.2 & 194.63 & ,206.47 \\
\hline 17 & 210 & 210 & 210 & 0 & 210.00 & ,210.00 \\
\hline 18 & 35 & 70 & 66.5 & 10.55 & 64.43 & 68.57 \\
\hline 19 & 210 & 210 & 210 & 0 & 210.00 & ,210.00 \\
\hline 20 & 42 & 168 & 164.64 & 19.51 & 160.82 & , 168.46 \\
\hline 21 & 10 & 120 & 39.3 & 34.06 & 32.62 &, 45.98 \\
\hline 22 & 105 & 210 & 201.6 & 28.63 & 195.99 & ,207.21 \\
\hline 23 & 210 & 210 & 210 & 0 & 210.00 & ,210.00 \\
\hline 24 & 35 & 70 & 64.4 & 12.9 & 61.87 & 66.93 \\
\hline 25 & 42 & 168 & 154.98 & 36.16 & 147.89 & ,162.07 \\
\hline 26 & 105 & 210 & 196.35 & 35.49 & 189.39 & ,203.31 \\
\hline 27 & 70 & 70 & 70 & 0 & 70.00 &, 70.00 \\
\hline 28 & 30 & 300 & 186.15 & 54.44 & 175.48 & , 196.82 \\
\hline 29 & 210 & 210 & 210 & 0 & 210.00 & ,210.00 \\
\hline 30 & 7 & 84 & 27.09 & 17.93 & 23.58 &, 30.60 \\
\hline 31 & 210 & 210 & 210 & 0 & 210.00 & ,210.00 \\
\hline 32 & 105 & 210 & 193.2 & 38.69 & 185.62 & ,200.78 \\
\hline 33 & 70 & 70 & 70 & 0 & 70.00 &, 70.00 \\
\hline 34 & 105 & 210 & 192.15 & 39.64 & 184.38 & ,199.92 \\
\hline 35 & 6 & 24 & 10.74 & 7.64 & 9.24 & , 12.24 \\
\hline 36 & 35 & 70 & 67.55 & 8.98 & 65.79 &, 69.31 \\
\hline 37 & 210 & 210 & 210 & 0 & 210.00 & ,210.00 \\
\hline 38 & 105 & 210 & 204.75 & 23 & 200.24 & ,209.26 \\
\hline 39 & 70 & 70 & 70 & 0 & 70.00 &, 70.00 \\
\hline 40 & 21 & 168 & 152.88 & 41.47 & 144.75 & ,161.01 \\
\hline 41 & 210 & 210 & 210 & 0 & 210.00 & ,210.00 \\
\hline 42 & 5 & 120 & 30.85 & 20.89 & 26.76 & ,34.94 \\
\hline 43 & 210 & 210 & 210 & 0 & 210.00 & ,210.00 \\
\hline 44 & 105 & 210 & 202.65 & 26.93 & 197.37 & ,207.93 \\
\hline 45 & 14 & 84 & 26.88 & 16.73 & 23.60 & ,30.16 \\
\hline 46 & 105 & 210 & 199.5 & 31.66 & 193.29 & ,205.71 \\
\hline 47 & 210 & 210 & 210 & 0 & 210.00 & ,210.00 \\
\hline 48 & 35 & 70 & 68.25 & 7.67 & 66.75 & 69.75 \\
\hline 49 & 30 & 300 & 195 & 66.67 & 181.93 & ,208.07 \\
\hline 50 & 21 & 168 & 156.45 & 35.49 & 149.49 & , 163.41 \\
\hline 51 & 70 & 70 & 70 & 0 & 70.00 & ,70.00 \\
\hline 52 & 105 & 210 & 197.4 & 34.29 & 190.68 & ,204.12 \\
\hline
\end{tabular}

TABLE C.5: Periodicities obtained by applying rotor-router on a random Clover tree with 106 nodes, and 100 random initial configurations for each set of $k=1, \ldots, 52$. 


\begin{tabular}{|c|c|c|c|c|c|c|}
\hline $\mathbf{K}$ & $\begin{array}{l}\text { Minimum } \\
\text { Periodicity }\end{array}$ & $\begin{array}{l}\text { Maximum } \\
\text { Periodicity }\end{array}$ & $\begin{array}{l}\text { Average } \\
\text { Periodicity }\end{array}$ & $\begin{array}{l}\text { Standard } \\
\text { Deviation }\end{array}$ & \multicolumn{2}{|c|}{$\begin{array}{c}\text { 95\% Confidence } \\
\text { Interval }\end{array}$} \\
\hline 53 & 210 & 210 & 210 & 0 & 210.00 & 210.00, \\
\hline 54 & 35 & 70 & 68.6 & 6.89 & 67.25 & 69.95 \\
\hline 55 & 42 & 168 & 154.56 & 38.63 & 146.99 & 162.13, \\
\hline 56 & 15 & 300 & 200.25 & 64.36 & 187.63 & ,212.87 \\
\hline 57 & 70 & 70 & 70 & 0 & 70.00 & ,70.00 \\
\hline 58 & 105 & 210 & 204.75 & 23 & 200.24 & ,209.26 \\
\hline 59 & 210 & 210 & 210 & 0 & 210.00 & 210.00 \\
\hline 60 & 7 & 84 & 24.85 & 15.36 & 21.84 & ,27.86 \\
\hline 61 & 210 & 210 & 210 & 0 & 210.00 & 210.00 \\
\hline 62 & 105 & 210 & 197.4 & 34.29 & 190.68 & ,204.12 \\
\hline 63 & 10 & 120 & 38.9 & 27.19 & 33.57 & ,44.23 \\
\hline 64 & 105 & 210 & 207.9 & 14.77 & 205.00 & 210.80, \\
\hline 65 & 42 & 168 & 151.2 & 42.21 & 142.93 & 159.47, \\
\hline 66 & 35 & 70 & 68.95 & 6 & 67.77 & ,70.13 \\
\hline 67 & 210 & 210 & 210 & 0 & 210.00 & 210.00 \\
\hline 68 & 105 & 210 & 201.6 & 28.63 & 195.99 & ,207.21 \\
\hline 69 & 70 & 70 & 70 & 0 & 70.00 &, 70.00 \\
\hline 70 & 3 & 24 & 10.5 & 7.76 & 8.98 & 12.02 \\
\hline 71 & 210 & 210 & 210 & 0 & 210.00 & 210.00 \\
\hline 72 & 35 & 70 & 67.9 & 8.35 & 66.26 & ,69.54 \\
\hline 73 & 210 & 210 & 210 & 0 & 210.00 & ,210.00 \\
\hline 74 & 105 & 210 & 207.9 & 14.77 & 205.00 & ,210.80 \\
\hline 75 & 14 & 84 & 26.32 & 18.59 & 22.68 & ,29.96 \\
\hline 76 & 105 & 210 & 205.8 & 20.68 & 201.75 & 209.85, \\
\hline 77 & 60 & 300 & 198.6 & 52.36 & 188.34 & ,208.86 \\
\hline 78 & 35 & 70 & 68.6 & 6.89 & 67.25 & ,69.95 \\
\hline 79 & 210 & 210 & 210 & 0 & 210.00 & 210.00 \\
\hline 80 & 21 & 168 & 155.61 & 37.65 & 148.23 & 162.99, \\
\hline 81 & 70 & 70 & 70 & 0 & 70.00 & ,70.00 \\
\hline 82 & 105 & 210 & 205.8 & 20.68 & 201.75 & 209.85 \\
\hline 83 & 210 & 210 & 210 & 0 & 210.00 & 210.00 \\
\hline 84 & 5 & 120 & 34.2 & 25.66 & 29.17 & ,39.23 \\
\hline 85 & 42 & 168 & 165.9 & 15.07 & 162.95 & 168.85, \\
\hline 86 & 105 & 210 & 200.55 & 30.2 & 194.63 & ,206.47 \\
\hline 87 & 70 & 70 & 70 & 0 & 70.00 & ,70.00 \\
\hline 88 & 105 & 210 & 205.8 & 20.68 & 201.75 & 209.85 \\
\hline 89 & 210 & 210 & 210 & 0 & 210.00 & 210.00 \\
\hline 90 & 7 & 84 & 25.27 & 14.89 & 22.35 & ,28.19 \\
\hline 91 & 30 & 300 & 200.7 & 64.9 & 187.98 & ,213.42 \\
\hline 92 & 105 & 210 & 202.65 & 26.93 & 197.37 & 207.93 \\
\hline 93 & 70 & 70 & 70 & 0 & 70.00 & ,70.00 \\
\hline 94 & 105 & 210 & 202.65 & 26.93 & 197.37 & ,207.93 \\
\hline 95 & 42 & 168 & 150.36 & 43.94 & 141.75 & 158.97, \\
\hline 96 & 35 & 70 & 68.25 & 7.67 & 66.75 & ,69.75 \\
\hline 97 & 210 & 210 & 210 & 0 & 210.00 & 210.00, \\
\hline 98 & 15 & 300 & 200.25 & 63.08 & 187.89 & ,212.61 \\
\hline 99 & 70 & 70 & 70 & 0 & 70.00 &, 70.00 \\
\hline 100 & 21 & 168 & 155.82 & 35.84 & 148.80 & ,162.84 \\
\hline 101 & 210 & 210 & 210 & 0 & 210.00 & 210.00 \\
\hline 102 & 35 & 70 & 67.2 & 9.54 & 65.33 & 69.07 \\
\hline 103 & 210 & 210 & 210 & 0 & 210.00 & ,210.00 \\
\hline 104 & 105 & 210 & 202.65 & 26.93 & 197.37 & ,207.93 \\
\hline 105 & 2 & 2 & 2 & 0 & 2.00 & ,2.00 \\
\hline
\end{tabular}

TABLE C.6: Periodicities obtained by applying rotor-router on a random Clover tree with 106 nodes, and 100 random initial configurations for each set of $k=53, \ldots, 105$. 


\begin{tabular}{|c|c|c|c|c|c|c|}
\hline \multirow[b]{2}{*}{$\mathbf{K}$} & \multirow{2}{*}{$\begin{array}{l}\text { Minimum } \\
\text { IdleTime }\end{array}$} & \multirow{2}{*}{$\begin{array}{l}\text { Maximum } \\
\text { IdleTime }\end{array}$} & \multirow{2}{*}{$\begin{array}{l}\text { Average } \\
\text { IdleTime }\end{array}$} & \multirow{2}{*}{$\begin{array}{l}\text { Standard } \\
\text { Deviation }\end{array}$} & \multirow{2}{*}{\multicolumn{2}{|c|}{$\begin{array}{c}\text { 95\% Confidence } \\
\text { Interval }\end{array}$}} \\
\hline & & & & & & \\
\hline 1 & 209 & 209 & 209 & 0 & 209.00 & 209.00 \\
\hline 2 & 104 & 139 & 119.89 & 9.53 & 118.02 & ,121.76 \\
\hline 3 & 69 & 69 & 69 & 0 & 69.00 & ,69.00 \\
\hline 4 & 52 & 55 & 52.68 & 0.8 & 52.52 &, 52.84 \\
\hline 5 & 41 & 43 & 42.37 & 0.58 & 42.26 & ,42.48 \\
\hline 6 & 35 & 38 & 35.14 & 0.47 & 35.05 & ,35.23 \\
\hline 7 & 29 & 32 & 30.46 & 0.66 & 30.33 & ,30.59 \\
\hline 8 & 26 & 27 & 26.05 & 0.22 & 26.01 & ,26.09 \\
\hline 9 & 23 & 25 & 23.07 & 0.29 & 23.01 & ,23.13 \\
\hline 10 & 21 & 22 & 21.09 & 0.29 & 21.03 & ,21.15 \\
\hline 11 & 19 & 19 & 19 & 0 & 19.00 & ,19.00 \\
\hline 12 & 17 & 18 & 17.44 & 0.5 & 17.34 & ,17.54 \\
\hline 13 & 16 & 17 & 16.24 & 0.43 & 16.16 & ,16.32 \\
\hline 14 & 15 & 16 & 15.1 & 0.3 & 15.04 & ,15.16 \\
\hline 15 & 13 & 14 & 13.58 & 0.5 & 13.48 & ,13.68 \\
\hline 16 & 13 & 13 & 13 & 0 & 13.00 & ,13.00 \\
\hline 17 & 13 & 13 & 13 & 0 & 13.00 & , 13.00 \\
\hline 18 & 11 & 12 & 11.99 & 0.1 & 11.97 & ,12.01 \\
\hline 19 & 11 & 11 & 11 & 0 & 11.00 & , 11.00 \\
\hline 20 & 11 & 11 & 11 & 0 & 11.00 & ,11.00 \\
\hline 21 & 9 & 11 & 9.67 & 0.49 & 9.57 & ,9.77 \\
\hline 22 & 9 & 10 & 9.99 & 0.1 & 9.97 & ,10.01 \\
\hline 23 & 9 & 9 & 9 & 0 & 9.00 & ,9.00 \\
\hline 24 & 9 & 9 & 9 & 0 & 9.00 & ,9.00 \\
\hline 25 & 9 & 9 & 9 & 0 & 9.00 & ,9.00 \\
\hline 26 & 8 & 9 & 8.02 & 0.14 & 7.99 & ,8.05 \\
\hline 27 & 8 & 8 & 8 & 0 & 8.00 & ,8.00 \\
\hline 28 & 8 & 8 & 8 & 0 & 8.00 & ,8.00 \\
\hline 29 & 7 & 8 & 7.73 & 0.45 & 7.64 & ,7.82 \\
\hline 30 & 7 & 7 & 7 & 0 & 7.00 & ,7.00 \\
\hline 31 & 7 & 7 & 7 & 0 & 7.00 & ,7.00 \\
\hline 32 & 7 & 7 & 7 & 0 & 7.00 & ,7.00 \\
\hline 33 & 7 & 7 & 7 & 0 & 7.00 & ,7.00 \\
\hline 34 & 7 & 7 & 7 & 0 & 7.00 & ,7.00 \\
\hline 35 & 5 & 6 & 5.31 & 0.46 & 5.22 &, 5.40 \\
\hline 36 & 6 & 6 & 6 & 0 & 6.00 & ,6.00 \\
\hline 37 & 6 & 6 & 6 & 0 & 6.00 & ,6.00 \\
\hline 38 & 6 & 6 & 6 & 0 & 6.00 & ,6.00 \\
\hline 39 & 5 & 6 & 5.95 & 0.22 & 5.91 & ,5.99 \\
\hline 40 & 5 & 6 & 5.83 & 0.38 & 5.76 &, 5.90 \\
\hline 41 & 5 & 5 & 5 & 0 & 5.00 &, 5.00 \\
\hline 42 & 5 & 6 & 5.02 & 0.14 & 4.99 & ,5.05 \\
\hline 43 & 5 & 5 & 5 & 0 & 5.00 &, 5.00 \\
\hline 44 & 5 & 5 & 5 & 0 & 5.00 & ,5.00 \\
\hline 45 & 5 & 5 & 5 & 0 & 5.00 & ,5.00 \\
\hline 46 & 5 & 5 & 5 & 0 & 5.00 &, 5.00 \\
\hline 47 & 5 & 5 & 5 & 0 & 5.00 & ,5.00 \\
\hline 48 & 5 & 5 & 5 & 0 & 5.00 & ,5.00 \\
\hline 49 & 5 & 5 & 5 & 0 & 5.00 & ,5.00 \\
\hline 50 & 5 & 5 & 5 & 0 & 5.00 &, 5.00 \\
\hline 51 & 4 & 5 & 4.81 & 0.39 & 4.73 & ,4.89 \\
\hline 52 & 4 & 5 & 4.04 & 0.2 & 4.00 & ,4.08 \\
\hline
\end{tabular}

TABLE C.7: Idle times obtained by applying rotor-router on a random Clover tree with 106 nodes, and 100 random initial configurations for each set of $k=1, \ldots, 52$. 


\begin{tabular}{|c|c|c|c|c|c|c|}
\hline K & $\begin{array}{l}\text { Minimum } \\
\text { IdleTime }\end{array}$ & $\begin{array}{l}\text { Maximum } \\
\text { IdleTime }\end{array}$ & $\begin{array}{l}\text { Average } \\
\text { IdleTime }\end{array}$ & $\begin{array}{l}\text { Standard } \\
\text { Deviation }\end{array}$ & \multicolumn{2}{|c|}{$\begin{array}{c}\text { 95\% Confidence } \\
\text { Interval }\end{array}$} \\
\hline 53 & 4 & 4 & 4 & 0 & 4.00 & ,4.00 \\
\hline 54 & 4 & 4 & 4 & 0 & 4.00 & 4.00 \\
\hline 55 & 4 & 4 & 4 & 0 & 4.00 & ,4.00 \\
\hline 56 & 4 & 4 & 4 & 0 & 4.00 & ,4.00 \\
\hline 57 & 4 & 4 & 4 & 0 & 4.00 & ,4.00 \\
\hline 58 & 4 & 4 & 4 & 0 & 4.00 & ,4.00 \\
\hline 59 & 4 & 4 & 4 & 0 & 4.00 & ,4.00 \\
\hline 60 & 4 & 4 & 4 & 0 & 4.00 & , 4.00 \\
\hline 61 & 4 & 4 & 4 & 0 & 4.00 & ,4.00 \\
\hline 62 & 4 & 4 & 4 & 0 & 4.00 & ,4.00 \\
\hline 63 & 3 & 4 & 3.78 & 0.42 & 3.70 & ,3.86 \\
\hline 64 & 3 & 4 & 3.92 & 0.27 & 3.87 & ,3.97 \\
\hline 65 & 3 & 4 & 3.9 & 0.3 & 3.84 & ,3.96 \\
\hline 66 & 3 & 4 & 3.6 & 0.49 & 3.50 & ,3.70 \\
\hline 67 & 3 & 4 & 3.59 & 0.49 & 3.49 & ,3.69 \\
\hline 68 & 3 & 4 & 3.41 & 0.49 & 3.31 & ,3.51 \\
\hline 69 & 3 & 3 & 3 & 0 & 3.00 & ,3.00 \\
\hline 70 & 3 & 3 & 3 & 0 & 3.00 & ,3.00 \\
\hline 71 & 3 & 3 & 3 & 0 & 3.00 & ,3.00 \\
\hline 72 & 3 & 3 & 3 & 0 & 3.00 & ,3.00 \\
\hline 73 & 3 & 3 & 3 & 0 & 3.00 & ,3.00 \\
\hline 74 & 3 & 3 & 3 & 0 & 3.00 & ,3.00 \\
\hline 75 & 3 & 3 & 3 & 0 & 3.00 & ,3.00 \\
\hline 76 & 3 & 3 & 3 & 0 & 3.00 & ,3.00 \\
\hline 77 & 3 & 3 & 3 & 0 & 3.00 & ,3.00 \\
\hline 78 & 3 & 3 & 3 & 0 & 3.00 & ,3.00 \\
\hline 79 & 3 & 3 & 3 & 0 & 3.00 & ,3.00 \\
\hline 80 & 3 & 3 & 3 & 0 & 3.00 & ,3.00 \\
\hline 81 & 3 & 3 & 3 & 0 & 3.00 & ,3.00 \\
\hline 82 & 3 & 3 & 3 & 0 & 3.00 & ,3.00 \\
\hline 83 & 3 & 3 & 3 & 0 & 3.00 & ,3.00 \\
\hline 84 & 3 & 3 & 3 & 0 & 3.00 & ,3.00 \\
\hline 85 & 3 & 3 & 3 & 0 & 3.00 & ,3.00 \\
\hline 86 & 3 & 3 & 3 & 0 & 3.00 & ,3.00 \\
\hline 87 & 3 & 3 & 3 & 0 & 3.00 & ,3.00 \\
\hline 88 & 3 & 3 & 3 & 0 & 3.00 & ,3.00 \\
\hline 89 & 3 & 3 & 3 & 0 & 3.00 & ,3.00 \\
\hline 90 & 3 & 3 & 3 & 0 & 3.00 & ,3.00 \\
\hline 91 & 3 & 3 & 3 & 0 & 3.00 & ,3.00 \\
\hline 92 & 3 & 3 & 3 & 0 & 3.00 & ,3.00 \\
\hline 93 & 3 & 3 & 3 & 0 & 3.00 & ,3.00 \\
\hline 94 & 3 & 3 & 3 & 0 & 3.00 & ,3.00 \\
\hline 95 & 3 & 3 & 3 & 0 & 3.00 & ,3.00 \\
\hline 96 & 3 & 3 & 3 & 0 & 3.00 & ,3.00 \\
\hline 97 & 3 & 3 & 3 & 0 & 3.00 & ,3.00 \\
\hline 98 & 3 & 3 & 3 & 0 & 3.00 & ,3.00 \\
\hline 99 & 3 & 3 & 3 & 0 & 3.00 & ,3.00 \\
\hline 100 & 3 & 3 & 3 & 0 & 3.00 & ,3.00 \\
\hline 101 & 3 & 3 & 3 & 0 & 3.00 & ,3.00 \\
\hline 102 & 2 & 3 & 2.69 & 0.46 & 2.60 & ,2.78 \\
\hline 103 & 2 & 3 & 2.89 & 0.31 & 2.83 & ,2.95 \\
\hline 104 & 2 & 2 & 2 & 0 & 2.00 & ,2.00 \\
\hline 105 & 1 & 1 & 1 & 0 & 1.00 & , 1.00 \\
\hline
\end{tabular}

TABLE C.8: Idle times obtained by applying rotor-router on a random Clover tree with 106 nodes, and 100 random initial configurations for each set of $k=53, \ldots, 105$. 


\section{Appendix D}

\section{Rotor-Router Experimental Results for a Tripod Tree}

The experimental results illustrated in Tables D.1, D.2, D.3, D.4, D.5, D.6, D.7, and D.8 are obtained by running experiments on the rotor-router simulator for a Tripod tree with 106 nodes, and 100 random initial configurations for each set of $k=1,2, \ldots, 105$ robots, i.e., 10,500 experiments were run in total. For the sake of clarity, we display the results obtained for each of the measurement parameters (cover time, lock-in time, periodicity, and idle time) in two separated tables: one for $k=1, \ldots, 52$, and another for $k=53, \ldots, 105$. 


\begin{tabular}{|c|c|c|c|c|c|c|}
\hline $\mathrm{K}$ & $\begin{array}{l}\text { Minimum } \\
\text { CoverTime }\end{array}$ & $\begin{array}{l}\text { Maximum } \\
\text { CoverTime }\end{array}$ & $\begin{array}{c}\text { Average } \\
\text { CoverTime }\end{array}$ & $\begin{array}{l}\text { Standard } \\
\text { Deviation }\end{array}$ & \multicolumn{2}{|c|}{$\begin{array}{c}95 \% \text { Confidence } \\
\text { Interval }\end{array}$} \\
\hline 1 & 1994 & 4132 & 3150.59 & 479.51 & 3056.61 & ,3244.57 \\
\hline 2 & 896 & 2588 & 1825.4 & 392.77 & 1748.42 & ,1902.38 \\
\hline 3 & 444 & 1894 & 1136.9 & 312.56 & 1075.64 & ,1198.16 \\
\hline 4 & 237 & 1512 & 821.07 & 303.77 & 761.53 & ,880.61 \\
\hline 5 & 186 & 1212 & 653.06 & 285.06 & 597.19 & ,708.93 \\
\hline 6 & 122 & 1168 & 469.32 & 220.55 & 426.09 &, 512.55 \\
\hline 7 & 120 & 1102 & 433.03 & 233.8 & 387.20 & ,478.86 \\
\hline 8 & 107 & 848 & 334.25 & 177.34 & 299.49 & ,369.01 \\
\hline 9 & 69 & 671 & 247.84 & 132.89 & 221.79 & 273.89 \\
\hline 10 & 67 & 1024 & 267.03 & 179.27 & 231.89 & ,302.17 \\
\hline 11 & 55 & 699 & 194.42 & 129.13 & 169.11 & ,219.73 \\
\hline 12 & 64 & 599 & 188.35 & 120.88 & 164.66 & ,212.04 \\
\hline 13 & 50 & 473 & 149.76 & 86.61 & 132.78 &, 166.74 \\
\hline 14 & 39 & 489 & 150.32 & 95.09 & 131.68 & ,168.96 \\
\hline 15 & 37 & 491 & 131.73 & 82.66 & 115.53 & ,147.93 \\
\hline 16 & 31 & 488 & 108.15 & 71.26 & 94.18 & ,122.12 \\
\hline 17 & 31 & 487 & 104.22 & 75.13 & 89.49 & ,118.95 \\
\hline 18 & 33 & 557 & 100 & 79.52 & 84.41 & ,115.59 \\
\hline 19 & 30 & 372 & 88.75 & 55.24 & 77.92 & ,99.58 \\
\hline 20 & 29 & 404 & 86.34 & 62.33 & 74.12 & ,98.56 \\
\hline 21 & 19 & 341 & 83.41 & 56.92 & 72.25 &, 94.57 \\
\hline 22 & 22 & 314 & 68.92 & 43.89 & 60.32 & ,77.52 \\
\hline 23 & 21 & 394 & 73.04 & 57.44 & 61.78 & ,84.30 \\
\hline 24 & 19 & 222 & 59.04 & 35.28 & 52.12 & ,65.96 \\
\hline 25 & 16 & 230 & 58.52 & 42.96 & 50.10 & ,66.94 \\
\hline 26 & 19 & 269 & 57.52 & 40.08 & 49.66 & ,65.38 \\
\hline 27 & 17 & 281 & 49.85 & 42.54 & 41.51 &, 58.19 \\
\hline 28 & 19 & 242 & 50.2 & 35.09 & 43.32 &, 57.08 \\
\hline 29 & 15 & 166 & 42.88 & 24.74 & 38.03 &, 47.73 \\
\hline 30 & 11 & 130 & 40.52 & 21.26 & 36.35 &, 44.69 \\
\hline 31 & 12 & 208 & 35.66 & 23.66 & 31.02 &, 40.30 \\
\hline 32 & 14 & 202 & 41.37 & 28.85 & 35.71 & ,47.03 \\
\hline 33 & 10 & 150 & 43.26 & 28.23 & 37.73 &, 48.79 \\
\hline 34 & 10 & 95 & 33.65 & 17.6 & 30.20 & ,37.10 \\
\hline 35 & 10 & 187 & 30.78 & 21.81 & 26.50 & ,35.06 \\
\hline 36 & 10 & 127 & 34.81 & 23.78 & 30.15 & ,39.47 \\
\hline 37 & 10 & 145 & 31 & 22.13 & 26.66 & ,35.34 \\
\hline 38 & 11 & 106 & 26.76 & 14.89 & 23.84 & ,29.68 \\
\hline 39 & 9 & 173 & 28.58 & 22.06 & 24.26 & ,32.90 \\
\hline 40 & 7 & 122 & 26.75 & 15.56 & 23.70 & ,29.80 \\
\hline 41 & 8 & 76 & 26.15 & 13.69 & 23.47 & ,28.83 \\
\hline 42 & 10 & 108 & 25.15 & 16.07 & 22.00 & ,28.30 \\
\hline 43 & 9 & 106 & 23.02 & 15.15 & 20.05 & ,25.99 \\
\hline 44 & 8 & 53 & 21.23 & 9.95 & 19.28 & ,23.18 \\
\hline 45 & 8 & 117 & 20.76 & 12.9 & 18.23 & ,23.29 \\
\hline 46 & 7 & 92 & 21.53 & 13.25 & 18.93 & ,24.13 \\
\hline 47 & 7 & 60 & 18.98 & 9.53 & 17.11 & ,20.85 \\
\hline 48 & 6 & 112 & 19.56 & 14.92 & 16.64 & ,22.48 \\
\hline 49 & 7 & 55 & 17.95 & 9.42 & 16.10 & ,19.80 \\
\hline 50 & 7 & 89 & 19.5 & 12.34 & 17.08 & ,21.92 \\
\hline 51 & 7 & 66 & 18.01 & 10.29 & 15.99 & ,20.03 \\
\hline 52 & 6 & 51 & 16.37 & 8.19 & 14.76 & ,17.98 \\
\hline
\end{tabular}

TABLE D.1: Cover times obtained by applying rotor-router on a Tripod tree with 106 nodes, and 100 random initial configurations for each set of $k=1, \ldots, 52$. 


\begin{tabular}{|c|c|c|c|c|c|c|}
\hline $\mathbf{K}$ & $\begin{array}{l}\text { Minimum } \\
\text { CoverTime }\end{array}$ & $\begin{array}{l}\text { Maximum } \\
\text { CoverTime }\end{array}$ & $\begin{array}{c}\text { Average } \\
\text { CoverTime }\end{array}$ & $\begin{array}{l}\text { Standard } \\
\text { Deviation }\end{array}$ & \multicolumn{2}{|c|}{$\begin{array}{c}\text { 95\% Confidence } \\
\text { Interval }\end{array}$} \\
\hline 53 & 5 & 43 & 15.93 & 7.27 & 14.51 & , 17.35 \\
\hline 54 & 6 & 59 & 15.56 & 7.97 & 14.00 & ,17.12 \\
\hline 55 & 7 & 44 & 16.33 & 7.48 & 14.86 & ,17.80 \\
\hline 56 & 6 & 64 & 15.29 & 8.85 & 13.55 & ,17.03 \\
\hline 57 & 6 & 48 & 13.74 & 6.92 & 12.38 & ,15.10 \\
\hline 58 & 5 & 67 & 15.2 & 9.92 & 13.26 & ,17.14 \\
\hline 59 & 5 & 63 & 13.87 & 7.74 & 12.35 & ,15.39 \\
\hline 60 & 5 & 37 & 13.08 & 6.08 & 11.89 & , 14.27 \\
\hline 61 & 5 & 68 & 14.31 & 9.39 & 12.47 & ,16.15 \\
\hline 62 & 5 & 97 & 12.77 & 9.88 & 10.83 & ,14.71 \\
\hline 63 & 6 & 38 & 12.92 & 5.88 & 11.77 & ,14.07 \\
\hline 64 & 4 & 69 & 12.29 & 8.14 & 10.69 & ,13.89 \\
\hline 65 & 6 & 43 & 12.28 & 6.15 & 11.07 & ,13.49 \\
\hline 66 & 5 & 39 & 11.82 & 6.23 & 10.60 & ,13.04 \\
\hline 67 & 5 & 30 & 11.66 & 5.36 & 10.61 & ,12.71 \\
\hline 68 & 4 & 46 & 11.82 & 6.52 & 10.54 & ,13.10 \\
\hline 69 & 4 & 29 & 10.36 & 4.51 & 9.48 & ,11.24 \\
\hline 70 & 4 & 46 & 11.38 & 6.04 & 10.20 & ,12.56 \\
\hline 71 & 5 & 36 & 10.39 & 5.1 & 9.39 & ,11.39 \\
\hline 72 & 4 & 41 & 9.84 & 4.91 & 8.88 & 10.80, \\
\hline 73 & 4 & 25 & 9.14 & 3.74 & 8.41 & ,9.87 \\
\hline 74 & 4 & 23 & 9.48 & 4.21 & 8.65 & ,10.31 \\
\hline 75 & 4 & 30 & 9.48 & 4.49 & 8.60 & ,10.36 \\
\hline 76 & 4 & 30 & 9.62 & 4.96 & 8.65 & 10.59, \\
\hline 77 & 3 & 36 & 9.09 & 5.11 & 8.09 & 10.09, \\
\hline 78 & 3 & 34 & 8.46 & 4.42 & 7.59 & ,9.33 \\
\hline 79 & 4 & 20 & 8.6 & 3.21 & 7.97 & ,9.23 \\
\hline 80 & 3 & 23 & 8.44 & 3.92 & 7.67 & ,9.21 \\
\hline 81 & 4 & 22 & 8.64 & 3.47 & 7.96 & ,9.32 \\
\hline 82 & 3 & 42 & 8.09 & 4.66 & 7.18 & ,9.00 \\
\hline 83 & 3 & 31 & 8.05 & 4.68 & 7.13 & ,8.97 \\
\hline 84 & 3 & 24 & 7.45 & 3.57 & 6.75 & ,8.15 \\
\hline 85 & 3 & 31 & 7.65 & 3.91 & 6.88 & ,8.42 \\
\hline 86 & 3 & 46 & 8.36 & 6.09 & 7.17 & ,9.55 \\
\hline 87 & 3 & 19 & 7.11 & 2.82 & 6.56 & ,7.66 \\
\hline 88 & 3 & 25 & 8.06 & 4.26 & 7.23 & 8.89 \\
\hline 89 & 3 & 23 & 7.33 & 3.58 & 6.63 & ,8.03 \\
\hline 90 & 3 & 53 & 7.35 & 5.49 & 6.27 & 8.43 \\
\hline 91 & 3 & 31 & 6.79 & 3.55 & 6.10 & 7.48 \\
\hline 92 & 3 & 25 & 6.98 & 3.42 & 6.31 & ,7.65 \\
\hline 93 & 3 & 36 & 7.28 & 5.25 & 6.25 & ,8.31 \\
\hline 94 & 3 & 27 & 7 & 3.59 & 6.30 & ,7.70 \\
\hline 95 & 3 & 19 & 6.3 & 2.7 & 5.77 & ,6.83 \\
\hline 96 & 3 & 24 & 6.71 & 3.45 & 6.03 & ,7.39 \\
\hline 97 & 3 & 25 & 6.44 & 3.19 & 5.82 & ,7.06 \\
\hline 98 & 3 & 12 & 6.01 & 2.08 & 5.60 &, 6.42 \\
\hline 99 & 3 & 23 & 5.95 & 3.08 & 5.35 & ,6.55 \\
\hline 100 & 3 & 14 & 5.81 & 2.03 & 5.41 & ,6.21 \\
\hline 101 & 3 & 13 & 5.74 & 2.28 & 5.29 &, 6.19 \\
\hline 102 & 3 & 17 & 6.09 & 2.81 & 5.54 &, 6.64 \\
\hline 103 & 3 & 16 & 6.05 & 2.49 & 5.56 & ,6.54 \\
\hline 104 & 2 & 18 & 5.44 & 2.8 & 4.89 & ,5.99 \\
\hline 105 & 2 & 21 & 5.63 & 2.54 & 5.13 & ,6.13 \\
\hline
\end{tabular}

TABLE D.2: Cover times obtained by applying rotor-router on a Tripod tree with 106 nodes, and 100 random initial configurations for each set of $k=53, \ldots, 105$. 


\begin{tabular}{|c|c|c|c|c|c|c|}
\hline $\mathbf{K}$ & $\begin{array}{c}\text { Minimum } \\
\text { Lock-inTime }\end{array}$ & $\begin{array}{c}\text { Maximum } \\
\text { Lock-inTime }\end{array}$ & $\begin{array}{c}\text { Average } \\
\text { Lock-inTime }\end{array}$ & $\begin{array}{l}\text { Standard } \\
\text { Deviation }\end{array}$ & \multicolumn{2}{|c|}{$\begin{array}{c}\text { 95\% Confidence } \\
\text { Interval }\end{array}$} \\
\hline 1 & 1996 & 4134 & 3152.32 & 479.25 & 3058.39 & ,3246.25 \\
\hline 2 & 1005 & 2699 & 1987.18 & 405.38 & 1907.72 & ,2066.64 \\
\hline 3 & 1767 & 6406 & 4729.53 & 1134.7 & 4507.13 & ,4951.93 \\
\hline 4 & 3369 & 9638 & 8375.61 & 908.11 & 8197.62 & ,8553.60 \\
\hline 5 & 1054 & 4377 & 3472.27 & 628.63 & 3349.06 & ,3595.48 \\
\hline 6 & 1352 & 5797 & 4615.41 & 875.97 & 4443.72 & ,4787.10 \\
\hline 7 & 1220 & 4773 & 3700.36 & 717.26 & 3559.78 & ,3840.94 \\
\hline 8 & 2469 & 8745 & 7600.84 & 856.64 & 7432.94 & ,7768.74 \\
\hline 9 & 2503 & 5914 & 4621.85 & 711.94 & 4482.31 & ,4761.39 \\
\hline 10 & 2387 & 6051 & 4721.05 & 682.41 & 4587.30 & ,4854.80 \\
\hline 11 & 4987 & 8711 & 7412.11 & 644.77 & 7285.73 & ,7538.49 \\
\hline 12 & 2456 & 6242 & 5025.9 & 617.27 & 4904.91 &, 5146.89 \\
\hline 13 & 6540 & 9875 & 8485.15 & 732.12 & 8341.65 & ,8628.65 \\
\hline 14 & 2014 & 4976 & 3731.49 & 526.54 & 3628.29 & ,3834.69 \\
\hline 15 & 807 & 4225 & 3034.51 & 690.1 & 2899.25 & ,3169.77 \\
\hline 16 & 3404 & 7434 & 6264.37 & 670.87 & 6132.88 & ,6395.86 \\
\hline 17 & 4410 & 7623 & 6263.51 & 723.49 & 6121.71 & ,6405.31 \\
\hline 18 & 3518 & 6833 & 5407.15 & 618.11 & 5286.00 &, 5528.30 \\
\hline 19 & 4848 & 10272 & 8662.54 & 968.11 & 8472.79 & ,8852.29 \\
\hline 20 & 2275 & 5461 & 4503.13 & 659.35 & 4373.90 & ,4632.36 \\
\hline 21 & 913 & 4064 & 2832.76 & 653.82 & 2704.61 & 2960.91 \\
\hline 22 & 2919 & 7679 & 6122.72 & 770.08 & 5971.78 & ,6273.66 \\
\hline 23 & 4740 & 10352 & 8685.97 & 1062.17 & 8477.78 & 8894.16 \\
\hline 24 & 3071 & 6342 & 5332.49 & 596.62 & 5215.55 &, 5449.43 \\
\hline 25 & 1373 & 5983 & 4676.14 & 709.31 & 4537.12 & ,4815.16 \\
\hline 26 & 3391 & 9554 & 8161 & 892.27 & 7986.12 & ,8335.88 \\
\hline 27 & 1997 & 6852 & 5480.07 & 787.69 & 5325.68 &, 5634.46 \\
\hline 28 & 2510 & 5362 & 4291 & 667.87 & 4160.10 & ,4421.90 \\
\hline 29 & 5117 & 10131 & 8730.31 & 848.77 & 8563.95 & ,8896.67 \\
\hline 30 & 1323 & 4358 & 3299.42 & 635.2 & 3174.92 & ,3423.92 \\
\hline 31 & 3197 & 8075 & 6826.91 & 694.35 & 6690.82 & ,6963.00 \\
\hline 32 & 5412 & 9576 & 8163.24 & 761.04 & 8014.08 & 8312.40 \\
\hline 33 & 2967 & 6709 & 5507.39 & 722.19 & 5365.84 &, 5648.94 \\
\hline 34 & 6509 & 11171 & 9200.48 & 1204.52 & 8964.39 & ,9436.57 \\
\hline 35 & 611 & 3574 & 2420.79 & 626.23 & 2298.05 & ,2543.53 \\
\hline 36 & 3797 & 7394 & 5747.09 & 813.29 & 5587.69 &, 5906.49 \\
\hline 37 & 6666 & 10812 & 9276.73 & 842.87 & 9111.53 & ,9441.93 \\
\hline 38 & 3717 & 7466 & 6360.63 & 699.09 & 6223.61 & 6497.65 \\
\hline 39 & 3431 & 6895 & 5628.62 & 716.92 & 5488.10 &, 5769.14 \\
\hline 40 & 3401 & 6286 & 5236.13 & 547.13 & 5128.89 &, 5343.37 \\
\hline 41 & 3655 & 7678 & 6576.14 & 697.81 & 6439.37 & ,6712.91 \\
\hline 42 & 764 & 4288 & 3084.29 & 712.03 & 2944.73 & ,3223.85 \\
\hline 43 & 6601 & 9805 & 8461.67 & 688.3 & 8326.76 & ,8596.58 \\
\hline 44 & 5746 & 10715 & 9022.99 & 1012.71 & 8824.50 & ,9221.48 \\
\hline 45 & 1389 & 5019 & 3583.62 & 656.94 & 3454.86 & ,3712.38 \\
\hline 46 & 6724 & 11005 & 9056.68 & 953.67 & 8869.76 & ,9243.60 \\
\hline 47 & 4021 & 8183 & 6806.41 & 830.84 & 6643.56 & ,6969.26 \\
\hline 48 & 3889 & 6877 & 5570.63 & 647 & 5443.82 &, 5697.44 \\
\hline 49 & 1631 & 5750 & 4610.4 & 756.33 & 4462.16 &, 4758.64 \\
\hline 50 & 2082 & 6164 & 4866.93 & 659.95 & 4737.58 & ,4996.28 \\
\hline 51 & 4010 & 7604 & 6196.81 & 736.18 & 6052.52 & ,6341.10 \\
\hline 52 & 3107 & 9485 & 7879.53 & 980.1 & 7687.43 & 8071.63 \\
\hline
\end{tabular}

TABLE D.3: Lock-in times obtained by applying rotor-router on a Tripod tree with 106 nodes, and 100 random initial configurations for each set of $k=1, \ldots, 52$. 


\begin{tabular}{|c|c|c|c|c|c|c|}
\hline $\mathbf{K}$ & $\begin{array}{c}\text { Minimum } \\
\text { Lock-inTime }\end{array}$ & $\begin{array}{c}\text { Maximum } \\
\text { Lock-inTime }\end{array}$ & $\begin{array}{c}\text { Average } \\
\text { Lock-inTime }\end{array}$ & $\begin{array}{l}\text { Standard } \\
\text { Deviation }\end{array}$ & \multicolumn{2}{|c|}{$\begin{array}{c}95 \% \text { Confidence } \\
\text { Interval }\end{array}$} \\
\hline 53 & 2166 & 9634 & 7956.41 & 1097.58 & 7741.28 & ,8171.54 \\
\hline 54 & 3728 & 7338 & 6070.95 & 802.13 & 5913.73 & ,6228.17 \\
\hline 55 & 2272 & 6084 & 4831.7 & 756.83 & 4683.36 & ,4980.04 \\
\hline 56 & 2607 & 5738 & 4730.57 & 640.61 & 4605.01 & ,4856.13 \\
\hline 57 & 2755 & 6821 & 5604.61 & 683.62 & 5470.62 &, 5738.60 \\
\hline 58 & 3367 & 8227 & 6986.42 & 727.87 & 6843.76 & ,7129.08 \\
\hline 59 & 6619 & 11224 & 9492.21 & 839.66 & 9327.64 &, 9656.78 \\
\hline 60 & 1711 & 4717 & 3689.86 & 641.22 & 3564.18 & ,3815.54 \\
\hline 61 & 5172 & 10782 & 8919.09 & 1121.95 & 8699.19 & ,9138.99 \\
\hline 62 & 6515 & 10406 & 8593.76 & 721.97 & 8452.25 & ,8735.27 \\
\hline 63 & 1242 & 4478 & 3337.76 & 692.62 & 3202.01 & ,3473.51 \\
\hline 64 & 4864 & 8008 & 6804.92 & 629.63 & 6681.51 & ,6928.33 \\
\hline 65 & 2045 & 6643 & 5379.91 & 785.55 & 5225.94 & ,5533.88 \\
\hline 66 & 3823 & 7433 & 6008.47 & 578.95 & 5895.00 &, 6121.94 \\
\hline 67 & 3697 & 7804 & 6663.72 & 671.5 & 6532.11 & ,6795.33 \\
\hline 68 & 5510 & 11221 & 9568.44 & 974.05 & 9377.53 & ,9759.35 \\
\hline 69 & 3839 & 7219 & 6044.09 & 710.7 & 5904.79 & ,6183.39 \\
\hline 70 & 918 & 4060 & 2909.14 & 660.96 & 2779.59 & ,3038.69 \\
\hline 71 & 6602 & 11885 & 9714.46 & 1215.6 & 9476.20 & ,9952.72 \\
\hline 72 & 3552 & 6864 & 5909.68 & 662.57 & 5779.82 & ,6039.54 \\
\hline 73 & 6111 & 10085 & 8503.66 & 754.92 & 8355.70 & ,8651.62 \\
\hline 74 & 4959 & 8690 & 7347.15 & 740.7 & 7201.97 & ,7492.33 \\
\hline 75 & 2070 & 4857 & 3833.3 & 610.47 & 3713.65 & ,3952.95 \\
\hline 76 & 6930 & 10735 & 9250.78 & 911.26 & 9072.17 & 9429.39 \\
\hline 77 & 2349 & 6074 & 4786.92 & 656.21 & 4658.30 & ,4915.54 \\
\hline 78 & 3420 & 7407 & 6199.22 & 663.29 & 6069.21 & ,6329.23 \\
\hline 79 & 6629 & 10403 & 8820.72 & 642.03 & 8694.88 & ,8946.56 \\
\hline 80 & 3091 & 6501 & 5373.36 & 589.27 & 5257.86 & ,5488.86 \\
\hline 81 & 1869 & 7160 & 5807.97 & 847.46 & 5641.87 & ,5974.07 \\
\hline 82 & 4038 & 10920 & 9337.85 & 1155.39 & 9111.39 & ,9564.31 \\
\hline 83 & 5451 & 7939 & 6873.04 & 609.36 & 6753.61 & ,6992.47 \\
\hline 84 & 1130 & 4698 & 3481.06 & 596.18 & 3364.21 & ,3597.91 \\
\hline 85 & 2192 & 6427 & 5103.33 & 697.94 & 4966.53 &, 5240.13 \\
\hline 86 & 3313 & 11113 & 9434.52 & 1072.96 & 9224.22 & ,9644.82 \\
\hline 87 & 4383 & 7357 & 5975.35 & 660.71 & 5845.85 & ,6104.85 \\
\hline 88 & 3782 & 8516 & 7191.97 & 906.85 & 7014.23 & ,7369.71 \\
\hline 89 & 4514 & 8559 & 7239.2 & 740.48 & 7094.07 & ,7384.33 \\
\hline 90 & 1587 & 5071 & 3845.18 & 664.81 & 3714.88 & 3975.48 \\
\hline 91 & 2831 & 5618 & 4507.74 & 612.96 & 4387.60 & ,4627.88 \\
\hline 92 & 7601 & 10821 & 9397.83 & 739.08 & 9252.97 & ,9542.69 \\
\hline 93 & 4109 & 7236 & 6046.81 & 684.84 & 5912.58 & ,6181.04 \\
\hline 94 & 6411 & 9820 & 8547.55 & 712.37 & 8407.93 & ,8687.17 \\
\hline 95 & 3722 & 7079 & 5745.21 & 628.22 & 5622.08 &, 5868.34 \\
\hline 96 & 4388 & 7194 & 6110.8 & 601.57 & 5992.89 & ,6228.71 \\
\hline 97 & 6832 & 9859 & 8799.22 & 578.95 & 8685.75 & ,8912.69 \\
\hline 98 & 770 & 6454 & 5016.68 & 830.71 & 4853.86 &, 5179.50 \\
\hline 99 & 1877 & 7197 & 6038.87 & 903.56 & 5861.77 & ,6215.97 \\
\hline 100 & 2341 & 6182 & 4992.93 & 704.62 & 4854.82 &, 5131.04 \\
\hline 101 & 8102 & 11856 & 10116.15 & 736.32 & 9971.83 & ,10260.47 \\
\hline 102 & 3843 & 8318 & 6616.14 & 941.17 & 6431.67 & ,6800.61 \\
\hline 103 & 1896 & 6146 & 4745.71 & 948.61 & 4559.78 & ,4931.64 \\
\hline 104 & 4157 & 9264 & 7812.93 & 964.27 & 7623.93 & ,8001.93 \\
\hline 105 & 1091 & 3027 & 2151.06 & 449.56 & 2062.95 & 2239.17 \\
\hline
\end{tabular}

TABLE D.4: Lock-in times obtained by applying rotor-router on a Tripod tree with 106 nodes, and 100 random initial configurations for each set of $k=53, \ldots, 105$. 


\begin{tabular}{|c|c|c|c|c|c|c|}
\hline $\mathrm{K}$ & $\begin{array}{l}\text { Minimum } \\
\text { Periodicity }\end{array}$ & $\begin{array}{l}\text { Maximum } \\
\text { Periodicity }\end{array}$ & $\begin{array}{c}\text { Average } \\
\text { Periodicity }\end{array}$ & $\begin{array}{l}\text { Standard } \\
\text { Deviation }\end{array}$ & \multicolumn{2}{|c|}{$\begin{array}{c}\text { 95\% Confidence } \\
\text { Interval }\end{array}$} \\
\hline 1 & 210 & 210 & 210 & 0 & 210.00 & 210.00 \\
\hline 2 & 105 & 210 & 205.8 & 20.68 & 201.75 & 209.85 \\
\hline 3 & 70 & 70 & 70 & 0 & 70.00 & ,70.00 \\
\hline 4 & 105 & 210 & 170.1 & 51.22 & 160.06 & , 180.14 \\
\hline 5 & 42 & 84 & 57.96 & 20.49 & 53.94 &, 61.98 \\
\hline 6 & 35 & 70 & 59.5 & 16.12 & 56.34 &, 62.66 \\
\hline 7 & 30 & 30 & 30 & 0 & 30.00 & ,30.00 \\
\hline 8 & 105 & 210 & 182.7 & 46.29 & 173.63 & 191.77 \\
\hline 9 & 70 & 70 & 70 & 0 & 70.00 &, 70.00 \\
\hline 10 & 21 & 84 & 56.91 & 24.12 & 52.18 &, 61.64 \\
\hline 11 & 210 & 210 & 210 & 0 & 210.00 & 210.00 \\
\hline 12 & 35 & 70 & 63 & 14.07 & 60.24 &, 65.76 \\
\hline 13 & 210 & 210 & 210 & 0 & 210.00 & ,210.00 \\
\hline 14 & 15 & 30 & 27.45 & 5.66 & 26.34 & ,28.56 \\
\hline 15 & 14 & 14 & 14 & 0 & 14.00 & ,14.00 \\
\hline 16 & 105 & 210 & 198.45 & 33.02 & 191.98 & 204.92 \\
\hline 17 & 210 & 210 & 210 & 0 & 210.00 & 210.00 \\
\hline 18 & 35 & 70 & 61.95 & 14.8 & 59.05 &, 64.85 \\
\hline 19 & 210 & 210 & 210 & 0 & 210.00 & 210.00 \\
\hline 20 & 21 & 84 & 55.44 & 23.98 & 50.74 &, 60.14 \\
\hline 21 & 10 & 10 & 10 & 0 & 10.00 & ,10.00 \\
\hline 22 & 105 & 210 & 198.45 & 33.02 & 191.98 & 204.92 \\
\hline 23 & 210 & 210 & 210 & 0 & 210.00 & 210.00 \\
\hline 24 & 35 & 70 & 66.15 & 11.01 & 63.99 &, 68.31 \\
\hline 25 & 42 & 84 & 57.12 & 20.26 & 53.15 & ,61.09 \\
\hline 26 & 105 & 210 & 192.15 & 39.64 & 184.38 & 199.92 \\
\hline 27 & 70 & 70 & 70 & 0 & 70.00 & ,70.00 \\
\hline 28 & 15 & 30 & 28.35 & 4.72 & 27.43 & ,29.27 \\
\hline 29 & 210 & 210 & 210 & 0 & 210.00 & 210.00 \\
\hline 30 & 7 & 14 & 13.23 & 2.2 & 12.80 & ,13.66 \\
\hline 31 & 210 & 210 & 210 & 0 & 210.00 & ,210.00 \\
\hline 32 & 105 & 210 & 197.4 & 34.29 & 190.68 & 204.12 \\
\hline 33 & 70 & 70 & 70 & 0 & 70.00 & ,70.00 \\
\hline 34 & 105 & 210 & 195.3 & 36.62 & 188.12 & 202.48 \\
\hline 35 & 6 & 12 & 6.36 & 1.43 & 6.08 &, 6.64 \\
\hline 36 & 35 & 70 & 66.85 & 10.07 & 64.88 & ,68.82 \\
\hline 37 & 210 & 210 & 210 & 0 & 210.00 & 210.00 \\
\hline 38 & 105 & 210 & 207.9 & 14.77 & 205.00 & ,210.80 \\
\hline 39 & 70 & 70 & 70 & 0 & 70.00 & ,70.00 \\
\hline 40 & 21 & 84 & 55.65 & 22.61 & 51.22 & ,60.08 \\
\hline 41 & 210 & 210 & 210 & 0 & 210.00 & 210.00 \\
\hline 42 & 5 & 10 & 9.5 & 1.51 & 9.20 &, 9.80 \\
\hline 43 & 210 & 210 & 210 & 0 & 210.00 & 210.00 \\
\hline 44 & 105 & 210 & 194.25 & 37.68 & 186.86 & 201.64 \\
\hline 45 & 14 & 14 & 14 & 0 & 14.00 &, 14.00 \\
\hline 46 & 105 & 210 & 197.4 & 34.29 & 190.68 & 204.12 \\
\hline 47 & 210 & 210 & 210 & 0 & 210.00 & 210.00 \\
\hline 48 & 35 & 70 & 65.45 & 11.83 & 63.13 &, 67.77 \\
\hline 49 & 30 & 30 & 30 & 0 & 30.00 &, 30.00 \\
\hline 50 & 21 & 84 & 58.38 & 23.61 & 53.75 & ,63.01 \\
\hline 51 & 70 & 70 & 70 & 0 & 70.00 &, 70.00 \\
\hline 52 & 105 & 210 & 196.35 & 35.49 & 189.39 & ,203.31 \\
\hline
\end{tabular}

TABLE D.5: Periodicity obtained by applying rotor-router on a Tripod tree with 106 nodes, and 100 random initial configurations for each set of $k=1, \ldots, 52$. 


\begin{tabular}{|c|c|c|c|c|c|c|}
\hline $\mathrm{K}$ & $\begin{array}{l}\text { Minimum } \\
\text { Periodicity }\end{array}$ & $\begin{array}{l}\text { Maximum } \\
\text { Periodicity }\end{array}$ & $\begin{array}{l}\text { Average } \\
\text { Periodicity }\end{array}$ & $\begin{array}{l}\text { Standard } \\
\text { Deviation }\end{array}$ & \multicolumn{2}{|c|}{$\begin{array}{c}\text { 95\% Confidence } \\
\text { Interval }\end{array}$} \\
\hline 53 & 210 & 210 & 210 & 0 & 210.00 & ,210.00 \\
\hline 54 & 35 & 70 & 66.85 & 10.07 & 64.88 & ,68.82 \\
\hline 55 & 42 & 84 & 59.64 & 20.83 & 55.56 & ,63.72 \\
\hline 56 & 15 & 30 & 28.2 & 4.9 & 27.24 & ,29.16 \\
\hline 57 & 70 & 70 & 70 & 0 & 70.00 & ,70.00 \\
\hline 58 & 105 & 210 & 205.8 & 20.68 & 201.75 & 209.85, \\
\hline 59 & 210 & 210 & 210 & 0 & 210.00 & 210.00 \\
\hline 60 & 7 & 14 & 13.16 & 2.29 & 12.71 & ,13.61 \\
\hline 61 & 210 & 210 & 210 & 0 & 210.00 & 210.00 \\
\hline 62 & 105 & 210 & 198.45 & 33.02 & 191.98 & 204.92 \\
\hline 63 & 10 & 10 & 10 & 0 & 10.00 &, 10.00 \\
\hline 64 & 105 & 210 & 205.8 & 20.68 & 201.75 & 209.85 \\
\hline 65 & 42 & 84 & 58.38 & 20.59 & 54.34 & ,62.42 \\
\hline 66 & 35 & 70 & 67.2 & 9.54 & 65.33 & ,69.07 \\
\hline 67 & 210 & 210 & 210 & 0 & 210.00 & ,210.00 \\
\hline 68 & 105 & 210 & 204.75 & 23 & 200.24 & 209.26 \\
\hline 69 & 70 & 70 & 70 & 0 & 70.00 & ,70.00 \\
\hline 70 & 3 & 12 & 6.3 & 1.83 & 5.94 & ,6.66 \\
\hline 71 & 210 & 210 & 210 & 0 & 210.00 & 210.00 \\
\hline 72 & 35 & 70 & 67.55 & 8.98 & 65.79 & ,69.31 \\
\hline 73 & 210 & 210 & 210 & 0 & 210.00 & 210.00 \\
\hline 74 & 105 & 210 & 204.75 & 23 & 200.24 & 209.26 \\
\hline 75 & 14 & 14 & 14 & 0 & 14.00 &, 14.00 \\
\hline 76 & 105 & 210 & 203.7 & 25.06 & 198.79 & 208.61 \\
\hline 77 & 30 & 30 & 30 & 0 & 30.00 & ,30.00 \\
\hline 78 & 35 & 70 & 68.25 & 7.67 & 66.75 &, 69.75 \\
\hline 79 & 210 & 210 & 210 & 0 & 210.00 & 210.00 \\
\hline 80 & 21 & 84 & 57.12 & 21.54 & 52.90 & ,61.34 \\
\hline 81 & 70 & 70 & 70 & 0 & 70.00 &, 70.00 \\
\hline 82 & 105 & 210 & 197.4 & 34.29 & 190.68 & 204.12 \\
\hline 83 & 210 & 210 & 210 & 0 & 210.00 & 210.00 \\
\hline 84 & 5 & 10 & 9.55 & 1.44 & 9.27 & ,9.83 \\
\hline 85 & 42 & 84 & 57.12 & 20.26 & 53.15 & ,61.09 \\
\hline 86 & 105 & 210 & 201.6 & 28.63 & 195.99 & ,207.21 \\
\hline 87 & 70 & 70 & 70 & 0 & 70.00 & ,70.00 \\
\hline 88 & 105 & 210 & 204.75 & 23 & 200.24 & ,209.26 \\
\hline 89 & 210 & 210 & 210 & 0 & 210.00 & 210.00 \\
\hline 90 & 7 & 14 & 13.37 & 2.01 & 12.98 &, 13.76 \\
\hline 91 & 30 & 30 & 30 & 0 & 30.00 & ,30.00 \\
\hline 92 & 105 & 210 & 203.7 & 25.06 & 198.79 & ,208.61 \\
\hline 93 & 70 & 70 & 70 & 0 & 70.00 &, 70.00 \\
\hline 94 & 105 & 210 & 201.6 & 28.63 & 195.99 & ,207.21 \\
\hline 95 & 42 & 84 & 57.12 & 20.26 & 53.15 &, 61.09 \\
\hline 96 & 35 & 70 & 67.2 & 9.54 & 65.33 & ,69.07 \\
\hline 97 & 210 & 210 & 210 & 0 & 210.00 & 210.00 \\
\hline 98 & 15 & 30 & 28.2 & 4.9 & 27.24 & ,29.16 \\
\hline 99 & 70 & 70 & 70 & 0 & 70.00 & ,70.00 \\
\hline 100 & 21 & 84 & 60.48 & 21.58 & 56.25 &, 64.71 \\
\hline 101 & 210 & 210 & 210 & 0 & 210.00 & ,210.00 \\
\hline 102 & 35 & 70 & 68.25 & 7.67 & 66.75 &, 69.75 \\
\hline 103 & 210 & 210 & 210 & 0 & 210.00 & ,210.00 \\
\hline 104 & 105 & 210 & 198.45 & 33.02 & 191.98 & 204.92 \\
\hline 105 & 2 & 2 & 2 & 0 & 2.00 & ,2.00 \\
\hline
\end{tabular}

TABLE D.6: Periodicity obtained by applying rotor-router on a Tripod tree with 106 nodes, and 100 random initial configurations for each set of $k=53, \ldots, 105$. 


\begin{tabular}{|c|c|c|c|c|c|c|}
\hline $\mathrm{K}$ & $\begin{array}{l}\text { Minimum } \\
\text { IdleTime }\end{array}$ & $\begin{array}{l}\text { Maximum } \\
\text { IdleTime }\end{array}$ & $\begin{array}{l}\text { Average } \\
\text { IdleTime }\end{array}$ & $\begin{array}{l}\text { Standard } \\
\text { Deviation }\end{array}$ & \multicolumn{2}{|c|}{$\begin{array}{c}\text { 95\% Confidence } \\
\text { Interval }\end{array}$} \\
\hline 1 & 209 & 209 & 209 & 0 & 209.00 & ,209.00 \\
\hline 2 & 104 & 136 & 116.5 & 8.84 & 114.77 &, 118.23 \\
\hline 3 & 69 & 69 & 69 & 0 & 69.00 &, 69.00 \\
\hline 4 & 52 & 53 & 52.62 & 0.49 & 52.52 &, 52.72 \\
\hline 5 & 41 & 42 & 41.38 & 0.49 & 41.28 & ,41.48 \\
\hline 6 & 35 & 35 & 35 & 0 & 35.00 & ,35.00 \\
\hline 7 & 29 & 29 & 29 & 0 & 29.00 & ,29.00 \\
\hline 8 & 26 & 27 & 26.31 & 0.46 & 26.22 & ,26.40 \\
\hline 9 & 23 & 23 & 23 & 0 & 23.00 & ,23.00 \\
\hline 10 & 21 & 21 & 21 & 0 & 21.00 & ,21.00 \\
\hline 11 & 19 & 19 & 19 & 0 & 19.00 & , 19.00 \\
\hline 12 & 17 & 18 & 17.84 & 0.37 & 17.77 & ,17.91 \\
\hline 13 & 16 & 17 & 16.22 & 0.42 & 16.14 &, 16.30 \\
\hline 14 & 15 & 15 & 15 & 0 & 15.00 & , 15.00 \\
\hline 15 & 13 & 13 & 13 & 0 & 13.00 & , 13.00 \\
\hline 16 & 13 & 13 & 13 & 0 & 13.00 & , 13.00 \\
\hline 17 & 13 & 13 & 13 & 0 & 13.00 & , 13.00 \\
\hline 18 & 12 & 12 & 12 & 0 & 12.00 & , 12.00 \\
\hline 19 & 11 & 11 & 11 & 0 & 11.00 & , 11.00 \\
\hline 20 & 11 & 11 & 11 & 0 & 11.00 & ,11.00 \\
\hline 21 & 9 & 9 & 9 & 0 & 9.00 & ,9.00 \\
\hline 22 & 9 & 10 & 9.97 & 0.17 & 9.94 & 10.00 \\
\hline 23 & 9 & 9 & 9 & 0 & 9.00 & ,9.00 \\
\hline 24 & 9 & 9 & 9 & 0 & 9.00 & 9.00 \\
\hline 25 & 9 & 9 & 9 & 0 & 9.00 &, 9.00 \\
\hline 26 & 8 & 9 & 8.07 & 0.26 & 8.02 & 8.12 \\
\hline 27 & 8 & 8 & 8 & 0 & 8.00 & ,8.00 \\
\hline 28 & 7 & 8 & 7.98 & 0.14 & 7.95 & ,8.01 \\
\hline 29 & 7 & 8 & 7.73 & 0.45 & 7.64 & ,7.82 \\
\hline 30 & 7 & 7 & 7 & 0 & 7.00 & ,7.00 \\
\hline 31 & 7 & 7 & 7 & 0 & 7.00 &, 7.00 \\
\hline 32 & 7 & 7 & 7 & 0 & 7.00 &, 7.00 \\
\hline 33 & 7 & 7 & 7 & 0 & 7.00 & ,7.00 \\
\hline 34 & 7 & 7 & 7 & 0 & 7.00 &, 7.00 \\
\hline 35 & 5 & 6 & 5.06 & 0.24 & 5.01 &, 5.11 \\
\hline 36 & 6 & 6 & 6 & 0 & 6.00 & 6.00 \\
\hline 37 & 6 & 6 & 6 & 0 & 6.00 & 6.00 \\
\hline 38 & 6 & 6 & 6 & 0 & 6.00 &, 6.00 \\
\hline 39 & 5 & 6 & 5.97 & 0.17 & 5.94 & 6.00 \\
\hline 40 & 5 & 6 & 5.9 & 0.3 & 5.84 &, 5.96 \\
\hline 41 & 5 & 6 & 5.49 & 0.5 & 5.39 &, 5.59 \\
\hline 42 & 5 & 5 & 5 & 0 & 5.00 &, 5.00 \\
\hline 43 & 5 & 5 & 5 & 0 & 5.00 &, 5.00 \\
\hline 44 & 5 & 5 & 5 & 0 & 5.00 &, 5.00 \\
\hline 45 & 5 & 5 & 5 & 0 & 5.00 &, 5.00 \\
\hline 46 & 5 & 5 & 5 & 0 & 5.00 &, 5.00 \\
\hline 47 & 5 & 5 & 5 & 0 & 5.00 &, 5.00 \\
\hline 48 & 5 & 5 & 5 & 0 & 5.00 &, 5.00 \\
\hline 49 & 5 & 5 & 5 & 0 & 5.00 &, 5.00 \\
\hline 50 & 5 & 5 & 5 & 0 & 5.00 &, 5.00 \\
\hline 51 & 5 & 5 & 5 & 0 & 5.00 &, 5.00 \\
\hline 52 & 4 & 5 & 4.02 & 0.14 & 3.99 & ,4.05 \\
\hline
\end{tabular}

TABLE D.7: Idle times obtained by applying rotor-router on a Tripod tree with 106 nodes, and 100 random initial configurations for each set of $k=1, \ldots, 52$. 


\begin{tabular}{|c|c|c|c|c|c|c|}
\hline $\mathrm{K}$ & $\begin{array}{l}\text { Minimum } \\
\text { IdleTime }\end{array}$ & $\begin{array}{l}\text { Maximum } \\
\text { IdleTime }\end{array}$ & $\begin{array}{l}\text { Average } \\
\text { IdleTime }\end{array}$ & $\begin{array}{l}\text { Standard } \\
\text { Deviation }\end{array}$ & \multicolumn{2}{|c|}{$\begin{array}{c}95 \% \text { Confidence } \\
\text { Interval }\end{array}$} \\
\hline 53 & 4 & 4 & 4 & 0 & 4.00 & ,4.00 \\
\hline 54 & 4 & 4 & 4 & 0 & 4.00 &, 4.00 \\
\hline 55 & 4 & 4 & 4 & 0 & 4.00 &, 4.00 \\
\hline 56 & 4 & 4 & 4 & 0 & 4.00 &, 4.00 \\
\hline 57 & 4 & 4 & 4 & 0 & 4.00 & ,4.00 \\
\hline 58 & 4 & 4 & 4 & 0 & 4.00 &, 4.00 \\
\hline 59 & 4 & 4 & 4 & 0 & 4.00 & ,4.00 \\
\hline 60 & 4 & 4 & 4 & 0 & 4.00 & ,4.00 \\
\hline 61 & 4 & 4 & 4 & 0 & 4.00 &, 4.00 \\
\hline 62 & 3 & 4 & 3.99 & 0.1 & 3.97 & ,4.01 \\
\hline 63 & 3 & 3 & 3 & 0 & 3.00 & ,3.00 \\
\hline 64 & 3 & 4 & 3.98 & 0.14 & 3.95 & ,4.01 \\
\hline 65 & 3 & 4 & 3.87 & 0.34 & 3.80 & ,3.94 \\
\hline 66 & 3 & 4 & 3.81 & 0.39 & 3.73 & ,3.89 \\
\hline 67 & 3 & 4 & 3.72 & 0.45 & 3.63 & ,3.81 \\
\hline 68 & 3 & 4 & 3.43 & 0.5 & 3.33 & ,3.53 \\
\hline 69 & 3 & 3 & 3 & 0 & 3.00 & ,3.00 \\
\hline 70 & 3 & 3 & 3 & 0 & 3.00 & ,3.00 \\
\hline 71 & 3 & 3 & 3 & 0 & 3.00 & ,3.00 \\
\hline 72 & 3 & 3 & 3 & 0 & 3.00 & ,3.00 \\
\hline 73 & 3 & 3 & 3 & 0 & 3.00 & ,3.00 \\
\hline 74 & 3 & 3 & 3 & 0 & 3.00 & ,3.00 \\
\hline 75 & 3 & 3 & 3 & 0 & 3.00 & ,3.00 \\
\hline 76 & 3 & 3 & 3 & 0 & 3.00 & ,3.00 \\
\hline 77 & 3 & 3 & 3 & 0 & 3.00 & ,3.00 \\
\hline 78 & 3 & 3 & 3 & 0 & 3.00 & ,3.00 \\
\hline 79 & 3 & 3 & 3 & 0 & 3.00 & ,3.00 \\
\hline 80 & 3 & 3 & 3 & 0 & 3.00 & ,3.00 \\
\hline 81 & 3 & 3 & 3 & 0 & 3.00 & ,3.00 \\
\hline 82 & 3 & 3 & 3 & 0 & 3.00 & ,3.00 \\
\hline 83 & 3 & 3 & 3 & 0 & 3.00 & ,3.00 \\
\hline 84 & 3 & 3 & 3 & 0 & 3.00 & ,3.00 \\
\hline 85 & 3 & 3 & 3 & 0 & 3.00 & ,3.00 \\
\hline 86 & 3 & 3 & 3 & 0 & 3.00 & ,3.00 \\
\hline 87 & 3 & 3 & 3 & 0 & 3.00 & ,3.00 \\
\hline 88 & 3 & 3 & 3 & 0 & 3.00 & ,3.00 \\
\hline 89 & 3 & 3 & 3 & 0 & 3.00 & ,3.00 \\
\hline 90 & 3 & 3 & 3 & 0 & 3.00 & ,3.00 \\
\hline 91 & 3 & 3 & 3 & 0 & 3.00 & ,3.00 \\
\hline 92 & 3 & 3 & 3 & 0 & 3.00 & ,3.00 \\
\hline 93 & 3 & 3 & 3 & 0 & 3.00 & ,3.00 \\
\hline 94 & 3 & 3 & 3 & 0 & 3.00 & ,3.00 \\
\hline 95 & 3 & 3 & 3 & 0 & 3.00 & ,3.00 \\
\hline 96 & 3 & 3 & 3 & 0 & 3.00 & ,3.00 \\
\hline 97 & 3 & 3 & 3 & 0 & 3.00 & ,3.00 \\
\hline 98 & 3 & 3 & 3 & 0 & 3.00 & ,3.00 \\
\hline 99 & 3 & 3 & 3 & 0 & 3.00 & ,3.00 \\
\hline 100 & 3 & 3 & 3 & 0 & 3.00 & ,3.00 \\
\hline 101 & 3 & 3 & 3 & 0 & 3.00 & ,3.00 \\
\hline 102 & 3 & 3 & 3 & 0 & 3.00 & ,3.00 \\
\hline 103 & 3 & 3 & 3 & 0 & 3.00 & ,3.00 \\
\hline 104 & 2 & 2 & 2 & 0 & 2.00 & ,2.00 \\
\hline 105 & 1 & 1 & 1 & 0 & 1.00 & , 1.00 \\
\hline
\end{tabular}

TABLE D.8: Idle times obtained by applying rotor-router on a Tripod tree with 106 nodes, and 100 random initial configurations for each set of $k=53, \ldots, 105$. 


\section{Abbreviations}

TSP Traveling Salesman Problem

UAV Unmanned Aerial Vehicle

CPT Chinese Postman Tour

3ECC 3-Edge-Coloring in Cubic Graphs 


\section{Nomenclature}

C Bidirectional Circle

$G \quad$ Arbitrary graph

I Idle Time

$S \quad$ Segment

$v_{i} \quad$ Speed of robot $i$

$T \quad$ Tree

$r_{i} \quad$ Visibility of robot $i$

$a_{i} \quad$ Robot $i$ from a set of robots

$D \quad$ Diameter of a graph

E Set of the edges of a graph 
$f \quad$ Number of faulty robots

$k \quad$ Number of robots

$m \quad$ Number of edges of a graph

$n \quad$ Number of nodes of a graph

$V \quad$ Set of the verticies of a graph 


\section{Bibliography}

[1] Robocup organization. URL http://www. robocup. org/.

[2] N. Agmon, N. Hazon, and G. A. Kaminka. The giving tree: constructing trees for efficient offline and online multi-robot coverage. Annals of Mathematics and Artificial Intelligence, 52(2):143-168, 2008.

[3] N. Agmon, S. Kraus, and G. A. Kaminka. Multi-robot perimeter patrol in adversarial settings. In Proceedings of IEEE International Conference on Robotics and Automation (ICRA 2008), pages 2339-2345, 2008.

[4] M. Ahmadi and P. Stone. A multi-robot system for continuous area sweeping tasks. In Proceedings of IEEE International Conference on Robotics and Automation (ICRA 2006), pages 1724-1729, 2006. 
[5] D. Aldous. The random walk construction of uniform spanning trees and uniform labeled trees. SIAM Journal on Discrete Mathematics, 3(4):450-465, 1990.

[6] A. Almeida, G. Ramalho, H. Santana, P. A. Tedesco, T. Menezes, V. Corruble, and Y. Chevaleyre. Recent advances on multi-agent patrolling. In Proceedings of 17th Brazilian Symposium on Artificial Intelligence, pages 474-483, 2004.

[7] S. Alpern, A. Morton, and K. Papadaki. Patrolling games. Operations Research, 59(5):1246-1257, 2011.

[8] F. Amigoni, N. Basilico, and N. Gatti. Finding the optimal strategies for robotic patrolling with adversaries in topologically-represented environments. In Proceedings of IEEE International Conference on Robotics and Automation (ICRA 2009), pages 819-824, 2009.

[9] F. Amigoni, N. Basilico, N. Gatti, A. Saporiti, and S. Troiani. Moving game theoretical patrolling strategies from theory to practice: an usarsim simulation. In IEEE International Conference on Robotics and Automation (ICRA 2010), pages 426-431, 2010. 
[10] R. C. Andrade, H. T. Macedo, G. L. Ramalho, and C. A. G. Ferraz. Distributed mobile autonomous agents in network management. In Proceedings of the International Conference on Parallel and Distributed Processing Techniques and Applications (PDPTA 2001), 2001.

[11] E. M. Arkin, J. S. B. Mitchell, and C. D. Piatko. Minimum-link watchman tours. Information Processing Letters, 86(4):203-207, 2003.

[12] E. Bampas, L. Gasieniec, N. Hanusse, D. Ilcinkas, R. Klasing, and A. Kosowski. Euler tour lock-in problem in the rotor-router model: i choose pointers and you choose port numbers. In Proceedings of the 23rd International Conference on Distributed Computing (DISC 2009), pages 423-435, 2009.

[13] E. Bampas, L. Gasieniec, R. Klasing, A. Kosowski, and T. Radzik. Robustness of the rotor-router mechanism. In Proceedings of 13th International Conference on On Principals Of Distributed Systems (OPODIS 2009), pages 345-358, 2009.

[14] J. Barajas and O. Serra. The lonely runner with seven runners. The Electronic Journal of Combinatorics, 15(1), 2008. 
[15] T. Bohman, R. Holzman, and D. Kleitman. Six lonely runners. The Electronic Journal of Combinatorics, 8(2), 2001.

[16] A. Broder. Generating random spanning trees. In 30th Annual Symposium on Foundations of Computer Science, pages 442-447, 1989.

[17] G. Cannata and A. Sgorbissa. A minimalist algorithm for multi-robot continuous coverage. IEEE Transactions on Robotics, 27(2):297-312, 2011.

[18] S. Carlsson, B. J. Nilsson, and S. C. Ntafos. Optimum guard covers and mwatchmen routes for restricted polygons. International Journal of Computational Geometry and Applications, 3(1):85-105, 1993.

[19] D. Carrolla, C. Nguyena, H. Everetta, and B. Frederickb. Development and testing for physical security robots. In Proceedings of SPIE Volume 5804, Unmanned Ground Vehicle Technology VII, pages 550-559, 2005.

[20] D. W. Casbeer, D. B. Kingston, R. W. Beard, T. W. Mclain, S. M. Li, and R. Mehra. Cooperative forest fire surveillance using a team of small unmanned air vehicles. International Journal of Systems Sciences, 37(6):351-360, 
2006.

[21] J. Chalopin, S. Das, P. Gawrychowski, A. Kosowski, A. Labourel, and P. Uznanski. Limit behavior of the multi-agent rotor-router system. In Proceedings of 29th International Symposium on Distributed Computing (DISC 2015), pages 123-139, 2015.

[22] Y. Chevaleyre. Theoretical analysis of the multi-agent patrolling problem. In Proceedings of International Conference on Intelligent Agent Technology (IAT 2004), pages 302-308, 2004.

[23] W. Chin and S. C. Ntafos. The zookeeper route problem. Information Sciences, 63(3):245-259, 1992.

[24] J. Cho and H. Garcia-Molina. Synchronizing a database to improve freshness. In Proceedings of the 2000 ACM SIGMOD International Conference on Management of Data (SIGMOD 2000), pages 117-128, 2000.

[25] J. Clark and R. Fierro. Mobile robotic sensors for perimeter detection and tracking. ISA Transaction, 46(1):3-13, 2007. 
[26] J. Colegrave and A. Branch. A case study of autonomous household vacuum cleaner. In AIAA/NASA Conference on Intelligent Robotics in Field, Factory, Service, and Space (CIRFFSS 1994), 1994.

[27] A. Collins, J. Czyzowicz, L. Gasieniec, A. Kosowski, E. Kranakis, D. Krizanc, R. Martin, and O. Morales Ponce. Optimal patrolling of fragmented boundaries. In Proceedings of the 25th Annual ACM Symposium on Parallelism in Algorithms and Architectures (SPAA 2013), pages 241-250, 2013.

[28] T. W. Cusick. View-obstruction problems. Aequationes Mathematicae, 9(2):165170, 1973.

[29] J. Czyzowicz, P. Egyed, H. Everett, D. Rappaport, T. C. Shermer, D. L. Souvaine, G. T. Toussaint, and J. Urrutia. The aquarium keeper's problem. In Proceedings of the Second Annual ACM-SIAM Symposium on Discrete Algorithms (SODA 1991), pages 459-464, 1991.

[30] J. Czyzowicz, L. Gasieniec, A. Kosowski, and E. Kranakis. Boundary patrolling by mobile agents with distinct maximal speeds. In Proceedings of 19th 
European Symposium on Algorithms (ESA 2011), pages 701-712, 2011.

[31] J. Czyzowicz, E. Kranakis, D. Pajak, and N. Taleb. Patrolling by robots equipped with visibility. In Proceedings of 21st International Colloquium, SIROCCO 2014, pages 224-234, 2014.

[32] J. Czyzowicz, L. Gasieniec, K. Georgiou, E. Kranakis, and F. MacQuarrie. The beachcombers' problem: walking and searching with mobile robots. Theoretical Computer Science, 608(3):201-218, 2015.

[33] J. Czyzowicz, L. Gasieniec, A. Kosowski, E. Kranakis, D. Krizanc, and N. Taleb. When patrolmen become corrupted: monitoring a graph using faulty mobile robots. In Proceedings of 26th International Symposium on Algorithms and Computation (ISAAC 2015), pages 343-354, 2015.

[34] J. Czyzowicz, K. Georgiou, E. Kranakis, F. MacQuarrie, and D. Pajak. Fence patrolling with two-speed robots. In Proceedings of 5th International Conference on Operations Research and Enterprse Systems (ICORES 2016), 2016. 
[35] J. Czyzowicz, L. Gasieniec, A. Kosowski, E. Kranakis, and N. Taleb. Optimal patrolling by mobile agents in arbitrary continuous graphs, In preparation.

[36] D. Dereniowski, A. Kosowski, D. Pajak, and P. Uznanski. Bounds on the cover time of parallel rotor walks. Journal of Computer and System Sciences, 82(5):8028016, 2016.

[37] M. Dror, A. Efrat, A. Lubiw, and J. S. B. Mitchell. Touring a sequence of polygons. In Proceedings of the 35th Annual ACM Symposium on Theory of Computing (STOC 2003), pages 473-482, 2003.

[38] A. Dumitrescu and C. D. Tóth. Watchman tours for polygons with holes. Computational Geometry: Theory and Applications, 45(7):326-333, 2012.

[39] A. Dumitrescu, A. Ghosh, and C. D. Tóth. On fence patrolling by mobile agents. The Electronic Journal of Combinatorics, 21(3), 2014.

[40] K. Easton and J. W. Burdick. A coverage algorithm for multi-robot boundary inspection. In Proceedings of IEEE International Conference on Robotics and Automation (ICRA 2005), pages 727-734, 2005. 
[41] S. Eidenbenz, C. Stamm, and P. Widmayer. Inapproximability results for guarding polygons and terrains. Algorithmica, 31(1):79-113, 2001.

[42] Y. Elmaliach, A. Shiloni, and G. A. Kaminka. Frequency-based multi-robot fence patrolling. Technical Report MAVERICK 2008/01, Bar Ilan University, Computer Science Department, 2008.

[43] Y. Elmaliach, A. Shiloni, and G. A. Kaminka. A realistic model of frequencybased multi-robot polyline patrolling. In Proceedings of the 7th International Joint Conference on Autonomous Agents and Multiagent Systems (AAMAS 2008), pages 63-70, 2008.

[44] Y. Elmaliach, N. Agmon, and G. A. Kaminka. Multi-robot area patrol under frequency constraints. Annals of Mathematics and Artificial Intelligence, 57(3): 293-320, 2009.

[45] Y. Elor and A. M. Bruckstein. Autonomous multi-agent cycle based patrolling. In Proceedings of 7th International Conference, ANTS 2010, pages 119-130, 2010. 
[46] F. V. Fomin and D. M. Thilikos. An annotated bibliography on guaranteed graph searching. Theoretical Computer Science, 399(3):236-245, 2008.

[47] F. V. Fomin, P. A. Golovach, A. Hall, M. Mihalák, E. Vicari, and P. Widmayer. How to guard a graph? Algorithmica, 61(4):839-856, 2011.

[48] T. Friedrich and T. Sauerwald. The cover time of deterministic random walks. In Proceeding of 16th Annual International Conference on Computing and Combinatorics (COCOON 2010), pages 130-139, 2010.

[49] M. R. Garey and D. S. Johnson. Computers and Intractability; A Guide to the Theory of NP-Completeness. W. H. Freeman and Company, 1990.

[50] S. K. Ghosh. Approximation algorithms for art gallery problems in polygons and terrains. Discrete Applied Mathematics, 158(6):718-722, 2010.

[51] A. Girard, A. Howell, and J. Hedrick. Border patrol and surveillance mission using multiple unmanned air vehicles. In Proceedings of the 43rd IEEE Conference on Decision and Control (CDC 2004), pages 620-625, 2004. 
[52] Y. Guo and Z. Qu. Coverage control for a mobile robot patrolling a dynamic and uncertain environment. In Proceedings of the 5th World Congress on Intelligent Control and Automation (WCICA 2004), pages 4899-4903, 2004.

[53] N. Hazon and G. Kaminka. On redundancy, efficiency, and robustness in coverage for multiple robots. Robotics and Autonomous Systems, 56(12):1102$1114,2008$.

[54] S. Hedberg. Robots cleaning up hazardous waste. AI Expert, 10(5):20-24, 1995.

[55] I. Holyer. The NP-completeness of edge-coloring. SIAM Journal on Computing, 10(4):718-720, 1981.

[56] K. Hwang, J. Lin, and H. Huang. Cooperative patrol planning of multi-robot systems by a competitive auction system. In ICROS-SICE International Joint Conference, pages 4359-4363, 2009.

[57] R. Jain. The Art of Computer Systems Performance Analysis: Techniques for Experimental Design, Measurement, Simulation, and Modeling. Wiley, 1991. 
[58] A. Kawamura and Y. Kobayashi. Fence patrolling by mobile agents with distinct speeds. Distributed Computing, 28(2):147-154, 2015.

[59] A. Kawamura and M. Soejima. Simple strategies versus optimal schedules in multi-agent patrolling. In Proceedings of 9th International Conference on Algorithms and Complexity Conference (CIAC 2015), pages 261-273, 2015.

[60] D. Kingston, R. W. Beard, and R. S. Holt. Decentralized perimeter surveillance using a team of uavs. IEEE Transactions on Robotics, 24(6):1394-1404, 2008.

[61] R. Klasing, A. Kosowski, D. Pajak, and T. Sauerwald. The multi-agent rotorrouter on the ring: a deterministic alternative to parallel random walks. In ACM Symposium on Principles of Distributed Computing (PODC 2013), pages 365-374, 2013.

[62] A. Kolling and S. Carpin. The graph-clear problem: definition, theoretical properties and its connections to multirobot aided surveillance. In Proceedings of International Conference on Intelligent Robots and Systems (IROS 2007), pages 1003-1008, 2007. 
[63] A. Kosowski. Private communication, 2016.

[64] A. Kosowski and D. Pajak. Does adding more agents make a difference? a case study of cover time for the rotor-router. In Proceedings of 41st International Colloquium on Automata, Languages, and Programming (ICALP 2014), pages 544$555,2014$.

[65] A. Kotzig. Hamilton graphs and hamilton circuits. In Theory of Graphs and its Applications, Proceedings of the Symposium held in Smolenice, pages 63-82, 1964.

[66] A. Machado, A. Almeida, G. Ramalho, J. D. Zucker, and A. Drogoul. Multiagent movement coordination in patrolling. In Proceedings of the 3rd International Conference on Computer and Game, pages 155-170, 2002.

[67] A. Machado, G. Ramalho, J. D. Zucker, and A. Drogoul. Multi-agent patrolling: an empirical analysis of alternative architectures. In Third International Workshop on Multi-Agent Based Simulation (MABS 2002), pages 155-170, 2002. 
[68] A. Marino, L. E. Parker, G. Antonelli, and F. Caccavale. Behavioral control for multirobot perimeter patrol: a finite state automata approach. In IEEE International Conference on Robotics and Automation (ICRA 2009), pages 831836, 2009.

[69] J. Mitchell. Geometric shortest paths and network optimization. In Handbook of Computational Geometry, pages 633-701. J.-R. Sack and J. Urrutia, Eds. Elsevier Science, 2000.

[70] S. C. Ntafos. Watchman routes under limited visibility. Computational Geometry, 1(3):149-170, 1992.

[71] J. O'Rourke. Art Gallery Theorems and Algorithms. Oxford University Press, 1987.

[72] J. H. Park and H. C. Kim. Dihamiltonian decomposition of regular graphs with degree three. In Proceedings of the 25th International Workshop on GraphTheoretic Concepts in Computer Science (WG 1999), pages 240-249, 1999. 
[73] F. Pasqualetti, A. Franchi, and F. Bullo. On optimal cooperative patrolling. In IEEE Conference on Decision and Control (CDC 2010), pages 7153-7158, 2010.

[74] F. Pasqualetti, J. W. Durham, and F. Bullo. Cooperative patrolling via weighted tours: performance analysis and distributed algorithms. IEEE transactions on robotics, 28(5):1181-1188, 2012.

[75] F. Pasqualetti, A. Franchi, and F. Bullo. On cooperative patrolling: optimal trajectories, complexity analysis, and approximation algorithms. IEEE Transaction on Robotics, 28(3):592-606, 2012.

[76] F. Pasqualetti, F. Zanella, J. R. Peters, M. Spindler, R. Carli, and F. Bullo. Camera network coordination for intruder detection. IEEE Transactions on Control Systems Technology, 22(5):1669-1683, 2014.

[77] D. Portugal and R. Rocha. Msp algorithm: multi-robot patrolling based on territory allocation using balanced graph partitioning. In Proceedings of the 2010 ACM Symposium on Applied Computing (SAC 2010), pages 1271-1276, 2010. 
[78] D. Portugal and R. Rocha. A survey on multi-robot patrolling algorithms. In Proceedings of Doctoral Conference on Computing, Electrical and Industrial Systems (DoCEIS 2011), pages 139-146, 2011.

[79] S. Ross. Simulation. Academic Press, 1997.

[80] A. Ryan, X. Xiao, S. Rathinam, J. Tisdale, M. Zennaro, D. Caveney, R. Sengupta, and K. Hedrick. A modular software infrastructure for distributed control of collaborating uavs. In AIAA Guidance, Navigation, and Control Conference and Exhibit, 2006.

[81] T. Sak, J. Wainer, and S. Goldenstein. Probabilistic multiagent patrolling. In Proceedings of the 19th Brazilian Symposium on Artificial Intelligence: Advances in Artificial Intelligence (SBIA 2008), pages 124-133, 2008.

[82] H. Santana, G. Ramalho, V. Corruble, and B. Ratitch. Multi-agent patrolling with reinforcement learning. In Proceedings of the 3rd International Joint Conference on Autonomous Agents and Multiagent Systems (AAMAS 2004), pages 1122-1129, 2004. 
[83] F. Sempé and A. Drogoul. Adaptive patrol for a group of robots. In Proceeding of the International Conference on Intelligent Robots and Systems (IROS 2003), pages 2865-2869, 2003.

[84] T. C. Shermer. Recent results in art galleries. Proceedings of the IEEE, 80(9): 1384-1399, 1992.

[85] S. L. Smith and D. Rus. Multi-robot monitoring in dynamic environments with guaranteed currency of observations. In IEEE Conference on Decision and Control (CDC 2010), pages 514-521, 2010.

[86] S. L. Smith, M. Schwager, and D. Rus. Persistent robotic tasks: Monitoring and sweeping in changing environments. IEEE Transactions on Robotics, 28(2): 410-426, 2012.

[87] S. Susca, S. Martinez, and F. Bullo. Monitoring environmental boundaries with a robotic sensor network. IEEE Transaction Control System Technology, 16 (2):288-296, 2008. 
[88] R. Sutton and A. Barto. Reinforcement Learning: An Introduction. MIT Press, 1998.

[89] I. A. Wagner, M. Lindenbaum, and A. M. Bruckstein. Smell as a computational resource - a lesson we can learn from the ants. In Proceedings of Fourth Israeli Symposium on Theory of Computing and Systems (ISTCS 1996), pages 219-230, 1996.

[90] I. A. Wagner, M. Lindenbaum, and A. M. Bruckstein. Efficiently searching a graph by a smell oriented vertex process. Annals of Mathematics and Artificial Intelligence, 24(1):211-223, 1998.

[91] I. A. Wagner, M. Lindenbaum, and A. M. Bruckstein. Distributed covering by ant-robots using evaporating traces. IEEE Transactions on Robotics and Automation, 15(5):918-933, 1999.

[92] I. A. Wagner, M. Lindenbaum, and A. M. Bruckstein. Mac vs. pc: Determinism and randomness as complementary approaches to robotic exploration of 
continuous unknown domains. The International Journal of Robotics Research, 19(1):12-31, 2000.

[93] K. Williams and J. Burdick. Multi-robot boundary coverage with plan revision. In Proceedings of IEEE International Conference on Robotics and Automation (ICRA 2006), pages 1716-1723, 2006.

[94] J. M. Wills. Zwei satze uber inhomogene diophantische approximation von irrationalzehlen. Monatshefte fur Mathematik, 71(3):263-269, 1967.

[95] M. Yamashita, H. Umemoto, I. Suzuki, and T. Kameda. Searching for mobile intruders in a polygonal region by a group of mobile searchers. Algorithmica, 31(2):208-236, 2001.

[96] V. Yanovski, I. A. Wagner, and A. M. Bruckstein. A distributed ant algorithm for efficiently patrolling a network. Algorithmica, 37(3):165-186, 2003. 


\section{Index}

Area patrolling, 13

Blind robots, 7

Boundary patrolling, 18

Centralized patrolling, 24

Chinese Postman Tour, 31

Close curve, 18

Clover tree (star graph), 91

Cover time, 86

Cyclic strategy, 13

Distributed patrolling, 24

Eulerian cycle, 16

Eulerian graph, 29

Even domain, 6
Exit port, 80

Geometric tree, 69

Graph tree, 69

Hamiltonian cycle, 16

Homogeneous domain, 6

Identical domain, 6

Idleness, 2

Initial configuration, 80

Lock-in time, 89

Longest path, 16

Mobile robots, 7

Oblivious robots, 7 
Off-line algorithm, 79

On-line algorithm, 79

Open curve, 18

Parallel rotor-routers, 79

Partition strategy, 13

Patrolling problem, 1

Perimeter patrolling, 18

Periodic behavior, 81

Periodicity, 89

Ports' pointers, 80

Random Initial Configurations, 102

Random number generator, 98

Randomized Patrolling, 22

Reliable robots, 7

Robots' locations, 80

Silent robots, 7
Simulation, 96

Simulation model, 96

Single rotor-router, 79

Stable state, 80

Star graph, 81

Static domain, 6

Sub-cycle decomposition, 81

Synchronized robots, 7

System state, 80

Tree, 67

Tripod tree (star graph), 91

Uniform random trees, 101

Unreliable (faulty) robots, 28

Visibility, 48 